Notfall Rettungsmed 2015 · 18:833-903 DOI 10.1007/s10049-015-0096-7

Online publiziert: 12. November 2015 (c) European Resuscitation Council (ERC), German Resuscitation Council (GRC), Austrian Resuscitation Council (ARC) 2015
A. Truhlár ${ }^{1,2}$ - C.D. Deakin ${ }^{3}$ J. Soar ${ }^{4}$ G.E.A. Khalifa ${ }^{5}$ A. Alfonzo ${ }^{6}$ - J.J.L.M. Bierens ${ }^{7}$. G. Bratteb $\varnothing^{8} \cdot$ H. Brugger ${ }^{9} \cdot$ J. Dunning ${ }^{10} \cdot$ S. Hunyadi-Antičević ${ }^{11} \cdot$ R.W. Koster ${ }^{12}$.

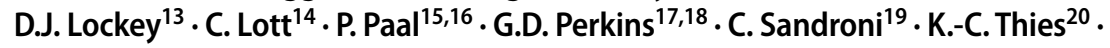
D.A. Zideman ${ }^{21}$. J.P. Nolan ${ }^{22}$

${ }^{1}$ Emergency Medical Services, Hradec Králové Region, Hradec Králové, Tschechien

2 Department of Anaesthesiology and Intensive Care Medicine, University Hospital Hradec Králové, Hradec Králové, Tschechien

${ }^{3}$ Cardiac Anaesthesia and Cardiac Intensive Care, NIHR Southampton Respiratory Biomedical Research Unit, Southampton University Hospital NHS Trust, Southampton, England

${ }^{4}$ Anaesthesia and Intensive Care Medicine, Southmead Hospital, North Bristol NHS Trust, Bristol, England

${ }^{5}$ Emergency and Disaster Medicine, Six October University Hospital, Cairo, Ägypten

${ }^{6}$ Departments of Renal and Internal Medicine, Victoria Hospital, Kirkcaldy, Fife, England

${ }^{7}$ Society to Rescue People from Drowning, Amsterdam, Niederlande

${ }^{8}$ Bergen Emergency Medical Services, Department of Anaesthesia and Intensive Care, Haukeland University Hospital, Bergen, Norwegen

${ }^{9}$ EURAC Institute of Mountain Emergency Medicine, Bozen, Italien

${ }^{10}$ Department of Cardiothoracic Surgery, James Cook University Hospital, Middlesbrough, England

${ }^{11}$ Centre for Emergency Medicine, Clinical Hospital Center Zagreb, Zagreb, Kroatien

12 Department of Cardiology, Academic Medical Center, Amsterdam, Niederlande

${ }^{13}$ Intensive Care Medicine and Anaesthesia, Southmead Hospital, North Bristol NHS Trust, Bristol, England

${ }^{14}$ Department of Anesthesiology, University Medical Center, Johannes Gutenberg-Universitaet, Mainz, Deutschland

${ }^{15}$ Barts Heart Centre, St Bartholomew's Hospital, Barts Health NHS Trus, Queen Mary University of London, London, England

${ }^{16}$ Department of Anaesthesiology and Critical Care Medicine, University Hospital Innsbruck, Innsbruck, Österreich

${ }^{17}$ Warwick Medical School, University of Warwick, Coventry, England

${ }^{18}$ Critical Care Unit, Heart of England NHS Foundation Trust, Birmingham, England

${ }^{19}$ Department of Anaesthesiology and Intensive Care, Catholic University School of Medicine, Rome, Italien

${ }^{20}$ Birmingham Children's Hospital, Birmingham, England

21 Department of Anaesthetics, Imperial College Healthcare NHS Trust, London, England

${ }^{22}$ Anaesthesia and Intensive Care Medicine, Royal United Hospital, UK and Bristol University, Bath, England

\section{Kreislaufstillstand in besonderen Situationen}

zu diesem Kapitel haben beigetragen:

Alessandro Barelli: Intensive Care Medicine and Clinical Toxicology, Catholic University School of Medicine, Rome, Italy

Bernd W. Böttiger: Department of Anaesthesiology and Intensive Care Medicine, University Hospital of Cologne, Cologne, Germany

Marios Georgiou: American Medical Center, Nicosia, Cyprus
Anthony J. Handley: Honorary Consultant Physician, Colchester, UK

Thomas Lindner: Department of Anaesthesiology and Intensive Care, Stavanger University Hospital, Stavanger, Norway; Norwegian Air Ambulance Foundation, Drøbak, Norway

Mark J. Midwinter: NIHR Surgical Reconstruction and Microbiology Research Centre, University of Birmingham, UK
Koenraad G. Monsieurs: Emergency Medicine, Faculty of Medicine and Health Sciences, University of Antwerp, Antwerp, Belgium and Faculty of Medicine and Health Sciences, University of Ghent, Ghent, Belgium Emergency Medicine, Ghent University, Ghent, Belgium

Wolfgang A. Wetsch: Department of Anaesthesiology and Intensive Care Medicine, University Hospital of Cologne, Cologne, Germany 


\section{Einführung}

Unabhängig von der Ursache des Kreislaufstillstands sind die wichtigsten Maßnahmen das frühe Erkennen und das adäquate Management des sich verschlechternden Patienten, der frühzeitige Notruf, die frühestmögliche Defibrillation, die qualitativ hochwertige kardiopulmonale Reanimation (CPR) mit kürzest möglicher Unterbrechung der Thoraxkompressionen sowie die Behandlung der reversiblen Ursachen. Unter bestimmten Umständen jedoch erfordern die Leitlinien zum „Advanced Life Support" (ALS) gewisse Anpassungen. Die folgenden "Leitlinien zur Wiederbelebung in besonderen Situationen" sind in drei Teile gegliedert: besondere Ursachen, besondere Umstände und besondere Patienten.

Der erste Teil befasst sich mit der Behandlung potenziell reversibler Ursachen des Kreislaufstillstands, für die es eine spezifische Behandlung gibt und die während jeder Reanimation erkannt oder ausgeschlossen werden müssen. Zum besseren Erinnern während der Reanimation werden diese in zwei Gruppen zu je vier Merkmalen unterteilt, die jeweils nach ihren Anfangsbuchstaben die „4 $4 \mathrm{H}^{\prime \prime}$ und „HITS“ genannt werden:

- die $4 \mathrm{H}$ sind Hypoxie, Hypo- oder Hyperkaliämie und andere Elektrolytstörungen, Hypo- oder Hyperthermie und Hypovolämie.

- Die HITS sind Herzbeuteltamponade, Intoxikation, Thrombose der Herz-

kranzgefäße oder der Lungenarterien und Spannungspneumothorax.

Der zweite Teil behandelt den Kreislaufstillstand unter besonderen Umständen, für die die universellen Leitlinien wegen spezifischer Örtlichkeiten des Kreislaufstillstands modifiziert werden müssen.

Und der dritte Teil konzentriert sich auf besondere Patienten mit speziellen Zuständen oder lange bestehenden Begleiterkrankungen, bei denen möglicherweise ein geändertes Vorgehen und unterschiedliche Therapieentscheidungen nötig sein können.

\section{Zusammenfassung der Änderungen gegenüber den Leitlinien von 2010}

Die wesentlichen Änderungen der vorliegenden Leitlinien gegenüber denen von 2010 [1] sind nachfolgend zusammengefasst:

\section{Spezielle Ursachen}

- Überleben nach einem asphyxiebedingten Kreislaufstillstand ist selten, und Überlebende haben oft schwere neurologische Beeinträchtigungen. Daher ist während der Reanimation die frühzeitige Beatmung unter Sauerstoffzugabe essenziell.

- Der Kreislaufstillstand durch Elektrolytstörungen kann durch ein hohes Maß an klinischem Argwohn und durch aggressives Vorgehen verhindert werden. Der neue Algorithmus liefert eine klinische Anleitung zur Behandlung der lebensbedrohlichen Hyperkaliämie.

- Unterkühlte Patienten ohne Zeichen einer Kreislaufinstabilität (systolischer Blutdruck $>90 \mathrm{mmHg}$, ohne ventrikulären Arrhythmien oder Kerntemperatur $>28^{\circ} \mathrm{C}$ ) können extern unter Verwendung minimal-invasiver Techniken (d. h. Warmluftgebläse und warme Infusionen) wiedererwärmt werden. Patienten mit Anzeichen kardialer Instabilität sollen direkt in ein Zentrum transportiert werden, das auf extrakorporale Herz- und Lungenunterstützung (ECLS) spezialisiert ist.

- Frühzeitiges Erkennen und sofortige Behandlung mit intramuskulärem Adrenalin bleiben die wichtigsten Säulen in der Notfallbehandlung der Anaphylaxie.

- Die Sterblichkeit des traumabedingten Kreislaufstillstands („trauma caused arrest“, TCA) ist sehr hoch. Die Hauptursache ist eine Blutung. Es ist anerkannt, dass die meisten Überlebenden keine Hypovolämie haben, sondern andere reversible Ursachen wie Hypoxie, Spannungspneumothorax oder Herzbeuteltamponade, die unverzüglich behandelt werden müssen. Der neue Behandlungsalgorithmus des TCA wurde entwickelt, um die Reihenfolge der lebensrettenden Maßnahmen zu priorisieren. Thoraxkompressionen sollen die Behandlung der reversiblen Ursachen nicht verzögern. Kreislaufstillstände nicht traumatischer Ursache, die sekundär zu einem Trauma geführt haben, müssen erkannt und nach Standardalgorithmen behandelt werden.

- Es gibt nur eine begrenzte Evidenz für die Empfehlung, Patienten mit einem präklinischen Kreislaufstillstand vermutlich kardialer Ursache unter laufender Reanimation zu transportieren. Dieses Vorgehen mag bei ausgewählten Patienten nutzbringend sein, wo sofortiger Zugang zu einer klinischen Herzkatheterintervention gegeben ist und wo eine Infrastruktur mit erfahrenen prähospitalen und innerklinischen Teams für die mechanische und hämodynamische Stabilisierung des Patienten und die perkutane Koronarintervention (PCI) unter laufender Reanimation besteht.

- Die Empfehlungen zur Fibrinolyse beim Verdacht auf Lungenembolie als Ursache des Kreislaufstillstands sind unverändert. Eine routinemäßige chirurgische Embolektomie oder mechanische Thrombektomie wird bei Verdacht auf Lungenembolie als Ursache des Kreislaufstillstands nicht empfohlen, soll aber in Erwägung gezogen werden, wenn die Diagnose einer Lungenembolie gesichert ist.

- Eine routinemäßige Magenspülung wird bei Vergiftungen nicht mehr empfohlen. Auch hat die Bedeutung der hyperbaren Sauerstofftherapie bei Kohlenmonoxidvergiftung abgenommen.

\section{Besondere Umgebung}

- Der Bereich „besondere Umgebung " beinhaltet Empfehlungen für die Behandlung des Kreislaufstillstands unter besonderen Umfeldbedingungen. Diese sind z. B. spezialisierte Gesundheitseinrichtungen, wie Operationssäle, Herzchirurgie, Katheterlabor, Dialyseein- 
heit, Zahn- und Kieferchirurgie, aber auch Passagier- und Ambulanzflugzeuge, Sportanlagen und Notfälle bei Outdoor-Aktivitäten, wie z. B. Ertrinken, unwegsames Gelände, Notfälle in großer Höhe, Lawinenverschüttung, Blitzschlag, Stromunfälle oder der Massenanfall von Notfallpatienten.

- Patienten, die sich chirurgischen Eingriffen unter Allgemeinanästhesie unterziehen, haben insbesondere im Notfall ein Risiko für einen perioperativen Kreislaufstillstand. Ein neuer Abschnitt in den Leitlinien deckt die üblichen Ursachen und wesentlichen Änderungen der Reanimationsmaßnahmen bei dieser Patientengruppe ab.

- Kreislaufstillstand nach großer Herzchirurgie ist relativ häufig in der unmittelbaren postoperativen Phase. Der Schlüssel zur erfolgreichen Wiederbelebung ist das schnelle Erkennen der Notwendigkeit einer Resternotomie, insbesondere bei Tamponade oder Blutung, wo externe Thoraxkompressionen wahrscheinlich ineffektiv sind. Die Resternotomie soll innerhalb von 5 min vorgenommen werden, nachdem andere Interventionen erfolglos waren.

- Kreislaufstillstand durch defibrillierbare Arrhythmien Kammerflimmern (VF) oder pulslose ventrikuläre Tachykardie (pVT) während Herzkatheterinterventionen soll durch drei sofort aufeinanderfolgende Defibrillationen vor dem Beginn von Thoraxkompressionen behandelt werden. Der Einsatz mechanischer Thoraxkompressionsgeräte während der Angiographie wird empfohlen, um hochwertige Thoraxkompressionen sicherzustellen und die Strahlenbelastung des Personals während der Koronarangiographie unter laufender Reanimation zu vermindern.

- In der Zahnarztpraxis soll der Patient für eine Reanimation nicht aus dem Zahnarztstuhl umgelagert werden. Vielmehr ist der Behandlungsstuhl schnell in eine horizontale Position zu bringen und das Kopfende mit einem untergestellten Hocker zu stabilisieren.

- Bei Anwendung automatisierter externer Defibrillatoren (AED) an Bord von kommerziellen Passagierflugzeugen beträgt die Überlebensrate bis zur Krankenhausentlassung bis zu $50 \%$. AED und adäquates Reanimationsequipment sollen daher verbindlich an Bord aller kommerziellen Flugzeuge in Europa vorgehalten werden, einschließlich Regional- und Billigfliegern. Wenn die Platzverhältnisse an Bord konventionelle Wiederbelebungsmethoden ausschließen, ist an die „Überkopfmethode“ der Reanimation zu denken.

- Die Inzidenz von Kreislaufstillständen an Bord von Rettungshubschraubern und Ambulanzflugzeugen ist gering. Dennoch werden die Wichtigkeit von vorbereitenden Maßnahmen vor dem Start und die Anwendung von mechanischen Thoraxkompressionsgeräten betont.

- Der plötzliche und unerwartete Kollaps eines Wettkämpfers auf der Sportanlage ist wahrscheinlich kardial bedingt und erfordert schnelles Erkennen und frühe Defibrillation.

- Die Dauer des Untertauchens ist ein bestimmender Faktor für das Outcome nach Ertrinken. Eine Submersionszeit von $>10$ min ist mit einem schlechten Outcome vergesellschaftet. Ersthelfer spielen daher eine wichtige Rolle in der frühen Rettung und Wiederbelebung von Ertrunkenen. Oxigenierung und Beatmung bilden weiterhin die Schwerpunkte in den Reanimationsbemühungen beim Atem- oder Kreislaufstillstand.

- Die Chancen für eine gute Erholung nach Kreislaufstillstand in unwegsamem Gelände oder in den Bergen können wegen des schwierigen $\mathrm{Zu}$ gangs und langer Transportzeiten vermindert sein. Daher spielen die Luftrettung und die Verfügbarkeit von AED in abgelegenen, aber viel besuchten Gebieten eine große Rolle.

- Die Abbruchkriterien für eine verlängerte Wiederbelebung und extrakorporale Wiedererwärmung bei
Lawinenopfern werden strenger gehandhabt, um die Anzahl aussichtsloser Fälle mit ECLS zu reduzieren. ECLS ist angezeigt bei einer Verschüttungsdauer von $>60$ min (bisher $>35 \mathrm{~min}$ ), wenn die Kerntemperatur bei Rettung $<30^{\circ} \mathrm{C}$ beträgt (bisher $<35^{\circ} \mathrm{C}$ ) und wenn das Serumkalium bei Klinikaufnahme $<8 \mathrm{mmol} / \mathrm{l}$ (bisher $<12 \mathrm{mmol} / \mathrm{l}$ ) beträgt.

- Es wird an die Beachtung von Sicherheitsmaßnahmen bei der Reanimation von Stromunfallopfern erinnert.

- Empfehlungen für die Behandlung von mehreren Notfallpatienten sollen Verzögerungen bei der Versorgung rettbarer Patienten bei einem Massenunfall vorbeugen. Ein Sichtungs- und Triagesystem soll benutzt werden, um die Reihenfolge der Versorgung festzulegen. Wenn die Anzahl der Unfallopfer die Ressourcen des Rettungsdienstes überschreitet, sollen keine Reanimationsmaßnahmen bei Patienten ohne Lebenszeichen unternommen werden.

\section{Spezielle Patienten}

- Der Abschnitt über spezielle Patienten bietet Hilfen zur Wiederbelebung von Patienten mit ernsthaften Begleiterkrankungen, wie z. B. Asthma, Herzinsuffizienz mit Herzunterstützungssystemen, neurologische Erkrankungen oder Fettleibigkeit und solchen mit physiologischen Besonderheiten, wie z. B. Schwangerschaft oder hohem Lebensalter.

- Die Therapie der ersten Wahl bei akutem Asthma sind inhalierbare $\beta_{2}$-Agonisten, während i.v.- $\beta_{2}-$ Agonisten nur für solche Patienten vorgeschlagen werden, bei denen die Inhalationstherapie nicht zuverlässig funktioniert. Inhalierbares Magnesium wird nicht mehr empfohlen.

- Bei Patienten mit Herzunterstützungssystemen („ventricular assist device“, VAD) kann die Feststellung eines Kreislaufstillstands schwierig sein. Wenn innerhalb der ersten 10 Tage nach Herzchirurgie 
Tab. 1 Gründe für den asphyktischen Kreislaufstillstand

Atemwegsverlegung durch Weichteile (Koma), Laryngospasmus, Aspiration

Anämie

Asthma bronchiale

Lawinenverschüttung

Zentrale Hypoventilation durch Schädel-HirnTrauma oder Rückenmarksverletzung

Chronisch obstruktive Atemwegserkrankung (COPD)

Ertrinken

Erhängen

Sauerstoffmangel in großer Höhe

Beeinträchtigte alveoläre Ventilation durch neuromuskuläre Erkrankungen

Pneumonie

Spannungspneumothorax

Trauma

Traumatisches oder Kompressions-Ersticken z. B. durch Druck innerhalb von Menschenmassen

ein Kreislaufstillstand nicht auf Defibrillation anspricht, soll sofort eine Resternotomie vorgenommen werden.

- Patienten mit einer Subarachnoidalblutung (SAB) können EKG-Veränderungen haben, die ein akutes Koronarsyndrom (ACS) vermuten lassen. Es ist daher von der klinischen Einschätzung der Wahrscheinlichkeit einer SAB vs. eines ACS abhängig, ob man eine Computertomographie (CT) vor oder nach einer Herzkatheterintervention veranlasst.

- Keine Änderungen in der Abfolge der Reanimationsmaßnahmen werden bei fettleibigen Patienten empfohlen, wenngleich hier effektive CPR eine große Herausforderung darstellt. Deshalb soll an einen häufigeren Wechsel der Helfer als im üblichen 2-minIntervall gedacht werden. Es wird die frühe endotracheale Intubation durch einen erfahrenen Anwender empfohlen.

- Für die schwangere Frau im Kreislaufstillstand werden weiterhin hochwertige CPR-Maßnahmen mit manueller Uterusverlagerung empfohlen, ebenso frühzeitige ALSMaßnahmen und die Entbindung des Fetus, wenn es nicht bald zum Wiedereinsetzen des Spontan- kreislaufs („return of spontaneous circulation“, ROSC) kommt.

\section{A. Spezielle Ursachen}

\section{Hypoxie}

\section{Einführung}

Kreislaufstillstand durch reine Hypoxämie ist ungewöhnlich. Häufiger sieht man ihn als Folge einer Erstickung, der häufigsten Ursache des nicht kardial bedingten Kreislaufstillstands. Es gibt viele Ursachen eines asphyxiebedingten Kreislaufstillstands (• Tab. 1). Obwohl üblicherweise eine Kombination von Hypoxämie und Hyperkapnie vorliegt, ist es letztlich die Hypoxämie, die den Kreislaufstillstand verursacht [2].

\section{Pathophysiologische Mechanismen}

Wenn die Atmung durch Atemwegsobstruktion komplett behindert wird oder eine Apnoe vorliegt, schwindet das Bewusstsein, wenn die Sauerstoffsättigung im arteriellen Blut ca. $60 \%$ erreicht. Wie lange dies dauert, ist schwierig vorauszusagen, aber es ist üblicherweise in 1-2 min der Fall [3]. In Tierexperimenten mit Kreislaufstillstand durch Ersticken kam es innerhalb von 3-11 min zur pulslosen elektrischen Aktivität (PEA). Asystolie folgt einige Minuten später [4]. Im Vergleich mit einer „einfachen "Apnoe verursachen übermäßige Atembemühungen bei Atemwegsobstruktion einen gesteigerten Sauerstoffverbrauch, der seinerseits in einer rapiden Sauerstoffentsättigung des arteriellen Blutes und damit einer kürzeren Zeit bis zum Kreislaufstillstand resultiert.

Nach Safar u. Paradis [2] führt eine komplette Atemwegsverlegung nach vorausgehender Luftatmung innerhalb von 5-10 min zum Kreislaufstillstand durch pulslose elektrische Aktivität. Kammerflimmern ist nur selten der erste nachgewiesene Rhythmus auf dem Monitor beim Kreislaufstillstand durch Ersticken. In einer der größten Serien von außerklinischen Kreislaufstillständen durch Erhängen aus Melbourne, Australien, hatten nur 7 von 1321 Patienten (0,5\%) Kammerflimmern [5].

\section{Behandlung}

Erste Priorität hat die Behandlung der Ursache von Asphyxie oder Hypoxämie, denn dies sind potenziell reversible Ursachen des Kreislaufstillstands. Das bessere Outcome präklinischer Kreislaufstillstände durch „Compressiononly-CPR“ trifft für Kreislaufstillstände durch Ersticken nicht zu [6]. Diese haben weit bessere Überlebensraten mit konventioneller Reanimation [7]. Folgen Sie bei der Reanimation dem Standard-ALSAlgorithmus.

\section{Ergebnisse}

Überleben nach Kreislaufstillstand durch Ersticken ist selten, und die meisten Überlebenden leiden unter schweren neurologischen Beeinträchtigungen. In fünf veröffentlichten Serien mit insgesamt 286 Patienten mit einem Kreislaufstillstand nach Erhängen wurde in nur 16\% der Fälle ein Reanimationsversuch unternommen. Von diesen überlebten $6(2 \%)$ mit voller Erholung, 11 andere Überlebende trugen bleibende schwere Hirnschäden davon [5, 8-11].

Bei einem Drittel (89; $31 \%)$ dieser 286 Patienten konnten die Rettungskräfte einen Spontankreislauf erzielen. Somit ist ROSC bei versuchter Reanimation dieser Patienten nicht ungewöhnlich, aber neurologisch unbeeinträchtigtes Überleben ist selten. Patienten, die bewusstlos sind, aber noch keinen Kreislaufstillstand erlitten haben, erreichen wahrscheinlich eine gute neurologische Erholung [8, 9, 12].

\section{Hypo-/Hyperkaliämie und andere Elektrolytstörungen}

\section{Einführung}

Elektrolytstörungen können Arrythmien oder einen Kreislaufstillstand verursachen. Lebensbedrohliche Arrythmien sind am häufigsten durch Kaliumstörungen bedingt, insbesondere durch Hyperkaliämie, seltener durch Calciumund Magnesiumabweichungen. Denken Sie an Elektrolytstörungen bei folgenden Risikogruppen: Nierenversagen, schwere Verbrennungen, Herzversagen und Diabetes mellitus.

Elektrolytwerte wurden definiert zur klinischen Entscheidungsfindung. Die 
exakten Werte, die bestimmte klinische Entscheidungen auslösen, hängen vom Zustand des Patienten und vom Verlauf der Laborwerte ab. Es gibt kaum Evidenz für die Behandlung von Elektrolytstörungen im Kreislaufstillstand. Man wendet hier die gleichen Strategien an wie bei Patienten ohne Kreislaufstillstand.

\section{Elektrolytstörungen vermeiden}

Identifizieren und behandeln Sie Elektrolytstörungen möglichst vor dem Auftreten eines Kreislaufstillstands. Überwachen Sie die Nierenfunktion bei Risikopatienten, und vermeiden Sie Medikamentenkombinationen, die eine Hyperkaliämie verschlimmern können. Verhindern Sie das Wiederauftreten von Elektrolytstörungen durch die Beseitigung der auslösenden Ursachen, wie Medikamente, Nahrungsmittel etc.

\section{Kaliumstörungen}

\section{Kaliumhaushalt}

Die extrazelluläre Kaliumkonzentration wird eng zwischen 3,5 und 5,0 mmol/l reguliert. Normalerweise besteht ein großer Konzentrationsgradient zwischen intrazellulären und extrazellulären Flüssigkeitskompartimenten. Dieser Kaliumgradient an Zellmembranen trägt zur Erregbarkeit von Nerven- und Muskelzellen bei, auch des Myokards. Die Untersuchung des Serumkaliums muss die Effekte von Verschiebungen des Serum-pH-Werts einbeziehen. Wenn der Serum-pH fällt (Azidämie), steigt das Serumkalium, weil sich Kalium aus den Zellen in den Gefäßraum verlagert. Dieser Prozess wird wieder umgekehrt, wenn der Serum-pH-Wert steigt (Alkaliämie.)

\section{Hyperkaliämie}

Hyperkaliämie ist die am häufigsten mit einem Kreislaufstillstand vergesellschaftete Elektrolytstörung. Sie wird üblicherweise durch eine beeinträchtigte Nierenfunktion, Medikamente oder eine gesteigerte Kaliumfreisetzung aus den Zellen und eine metabolische Azidose verursacht. Hyperkaliämie kommt bei etwa $10 \%$ aller Krankenhauspatienten vor [10-12].

Chronische Niereninsuffizienz ist in der allgemeinen Bevölkerung häufig, und die Inzidenz der Hyperkaliämie steigt von 2 auf $42 \%$, wenn die glomeruläre Filtrationsrate (GFR) von 60 auf $20 \mathrm{ml} /$ min abnimmt [13].

Patienten mit terminaler Niereninsuffizienz sind besonders gefährdet, insbesondere nach einem außerklinischen Kreislaufstillstands [14].

Langdauernde Hyperkaliämie ist ein unabhängiger Risikofaktor für die innerklinische Mortalität [15]. Akute Hyperkaliämie verursacht mit größerer Wahrscheinlichkeit Herzrhythmusstörungen oder Kreislaufstillstand als chronische Hyperkaliämie.

\section{Definition}

Es gibt keine allgemeingültige Definition für Hyperkaliämie. Die Autoren definieren den Begriff als eine Serumkaliumkonzentration $>5,5 \mathrm{mmol} / \mathrm{l}$. In der Praxis ist Hyperkaliämie ein Continuum. Wenn die Kaliumkonzentration über diesen Wert ansteigt, nimmt das Risiko für unerwünschte Ereignisse zu, und die Notwendigkeit einer dringlichen Behandlung steigt an. Schwere Hyperkaliämie wurde als eine Serumkaliumkonzentration $>6,5 \mathrm{mmol} / \mathrm{l}$ definiert.

\section{Ursachen}

Die Hauptursachen der Hyperkaliämie sind:

- Nierenversagen (d. h. akutes Nierenversagen oder chronische Nierenkrankheit),

- Medikamente (z. B. ACE-Hemmer, ATII-Antagonisten, kaliumsparende Diuretika, nicht-steroidale Entzündungshemmer, Beta-Blocker, Trimetoprim),

- Gewebeuntergang (Rhabdomyolyse, Tumorauflösung, Hämolyse),

- metabolische Azidose (z. B. Nierenversagen, diabetische Ketoazidose),

- endokrine Störungen (z. B. AddisonKrise),

- Diät (kann die alleinige Ursache sein bei Patienten mit fortgeschrittener chronischer Nierenerkrankung),

- fälschliche Pseudohyperkaliämie (verdächtig in Fällen mit normaler Nierenfunktion, normalem EKG und/oder einer Vorgeschichte von hämatologischen Störungen). Pseudohyperkaliämie beschreibt den
Befund eines erhöhten Serumkaliumwerts (geronnenes Blut) bei einem normalen Kaliumwert im Plasma (nicht geronnenes Blut). Der Gerinnungsprozess setzt Kalium aus den Zellen und Blutplättchen frei, was die Serumkaliumkonzentration um durchschnittlich 0,4 mmol/l ansteigen lässt. Die Hauptursache für Pseudohyperkaliämie sind lange Transportzeiten ins Labor oder schlechte Lagerungsbedingungen [16, 17].

Das Risiko für eine Hyperkaliämie steigt bei der Kombination von Faktoren, wie der gleichzeitigen Einnahme von ACEHemmern oder AT-II-Hemmern und kaliumsparenden Diuretika.

\section{Erkennen der Hyperkaliämie}

Schließen Sie eine Hyperkaliämie bei allen Patienten mit einer Arrhythmie oder einem Kreislaufstillstand aus. Patienten können eine Schwäche bis hin zu einer schlaffen Lähmung haben, ebenso Parästhesien oder abgeschwächte tiefe Muskelreflexe. Andererseits kann das klinische Bild auch durch die zugrunde liegende Krankheit, die die Hyperkaliämie verursacht hat, überlagert sein. Der erste Hinweis für eine Hyperkaliämie können auch EKG-Veränderungen, Arrythmien oder ein Kreislaufstillstand sein. Eine Blutgasanalyse kann Verzögerungen bei der Kaliummessung vermeiden helfen $[18,19]$.

Die Wirkung einer Hyperkaliämie auf das EKG hängt sowohl vom absoluten Serumkaliumwert ab als auch von der Geschwindigkeit des Anstiegs.

Die EKG-Veränderungen, die mit einer Hyperkaliämie in Verbindung zu bringen sind, sind üblicherweise fortschreitend und beinhalten:

- AV-Block ersten Grades (verlängertes PR-Intervall auf $>0,2 \mathrm{~s}$ ),

- flache oder fehlende P-Wellen,

- hohe, steile T-Wellen („Zeltform“).

Die T-Welle ist größer als die R-Welle in mehr als einer Ableitung,

- ST-Senkung,

- S- und T-Verschmelzungswelle (Sinuswelle),

- breiter QRS-Komplex (>0,12 s),

- ventrikuläre Tachykardie, 
- Bradykardie,

- Kreislaufstillstand (pulslose elektrische Aktivität, VF/pVT, Asystolie).

\section{Behandlung der Hyperkaliämie}

Die Behandlung der Hyperkaliämie beruht auf fünf Kernstrategien: [20]

- Schutz des Herzen,

- Kaliumverschiebung intrazellulär,

- Kaliumelimination aus dem Körper,

- Überwachung des Serumkaliums und des Blutzuckers,

- Verhinderung des Wiederauftretens.

Beginnen Sie die lebensrettende Behandlung, wenn ein starker Verdacht auf Hyperkaliämie besteht, z. B. wegen EKGVeränderungen, auch vor dem Vorliegen der Laborwerte. Die Behandlungsstrategie der Hyperkaliämie ist vielfach untersucht worden [21-23].

Folgen Sie dem Algorithmus der Notfallbehandlung der Hyperkaliämie. Vermeiden Sie eine SalbutamolMonotherapie, die ineffektiv sein kann. Es gibt auch nur eine ungenügende Evidenz für die Anwendung von Natriumbikarbonat zur Senkung des Serumkaliums. Denken Sie an die frühzeitige Verlegung auf die Intensivstation oder zu einem Spezialisten.

Die Hauptrisiken der Hyperkaliämiebehandlung sind:

- Hypoglykämie infolge GlukoseInsulin-Anwendung (üblicherweise 1-3 h nach Behandlungsbeginn, aber auch bis zu $6 \mathrm{~h}$ nach der Infusion [24]). Überwachen Sie den Blutzuckerspiegel, und behandeln Sie eine Hypoglykämie umgehend.

- Gewebenekrosen wegen paravenöser Calciumgabe. Vergewissern Sie sich deshalb, bevor sie Calcium geben, dass der i.v.-Zugang sicher ist.

- Intestinale Nekrosen oder Verlegungen infolge der Anwendung von kaliumaustauschenden Harzen. Vermeiden Sie daher deren längere Anwendung, und geben Sie Abführmittel.

- Wiederauftreten der Hyperkaliämie nach Abklingen der Wirkungen einer medikamentösen Therapie, d. h. innerhalb von 4-6 h. Überwachen Sie daher weiterhin das Serumkalium für mindestens $24 \mathrm{~h}$ nach einer Episode.

\section{Patient ohne Kreislaufstillstand}

Beurteilen Sie den Patienten:

- Verwenden Sie den systematischen ABCDE-Untersuchungsgang, und behandeln Sie jegliche Unregelmäßigkeiten.

- Legen Sie einen i.v.-Zugang.

- Überprüfen Sie das Serumkalium.

- Schreiben Sie ein EKG.

Überwachen Sie den Herzrhythmus bei Patienten mit schwerer Hyperkaliämie. Die Behandlung richtet sich nach der Schwere der Störung. Halten Sie sich dabei an den Notfallalgorithmus in

- Abb. 1

Leichte Erhöhung (5,5-5,9 mmol/l):

- Befassen Sie sich mit der Ursache der Hyperkaliämie, um diese zu korrigieren und einen weiteren Anstieg zu vermeiden (z. B. Medikamente, Nahrung).

- Wenn eine Behandlung angezeigt ist, entfernen Sie das Kalium aus dem Körper: geben Sie 15-30 g eines Kationenaustauschers als Calciumsalz, z. B. Calcium-Resonium ${ }^{\circledR}$-Pulver oder als Natriumsalz, z. B. Resonium ${ }^{\circledR}$-APulver, in 50-100 ml 20\%-Sorbitol [1] entweder oral oder als Retentionseinlauf (Wirkungseintritt nach $>4 \mathrm{~h}$ ).

\section{Mittelschwere Erhöhung (6,0-6,4 mmol/l)} ohne EKG-Veränderungen:

- Verschieben Sie Kalium intrazellulär mit einer Glukose-Insulin-Infusion: 10 Einheiten kurzwirksames Insulin auf $25 \mathrm{~g}$ Glukose i.v. über 15-30 min (Wirkungseintritt in 15-30 min, maximaler Effekt in 30-60 min, Wirkungsdauer 4-6 h, Blutzuckerspiegel überwachen!).

- Entfernen Sie Kalium aus dem Körper (s.o.; überlegen Sie Dialyse nach klinischer Möglichkeit).

Schwere Erhöhung ( $>$ 6,5 mmol/l) ohne EKG-Veränderungen:

- Nehmen Sie Expertenhilfe in Anspruch.

- Geben Sie Glukose-Insulin, wie oben.
- Vernebeln Sie 10-20 mg Salbutamol (Wirkungsbeginn in 15-30 min, Dauer 4-6 h).

- Kalium aus dem Körper entfernen (s. oben). Erwägen Sie eine Dialyse.

Schwere Erhöhung ( $>$ 6,5 $\mathrm{mmol} / \mathrm{l}) \mathrm{mit}$ toxischen EKG-Veränderungen:

- Schützen Sie das Herz mit Calciumchlorid: $10 \mathrm{ml}$ einer $10 \%$-Calciumchloridlösung i.v. über $2-5 \mathrm{~min}$, um die toxischen Effekte der Hyperkaliämie an der myokardialen Zellmembran zu antagonisieren. Dadurch wird das Risiko von Kammerflimmern oder pulsloser ventrikulärer Tachykardie vermindert, nicht aber das Serumkalium gesenkt (Wirkungseintritt nach 1-3 min).

- Setzen Sie kaliumverschiebende Medikamente ein (Glukose-Insulin und Salbutamol).

- Entfernen Sie Kalium aus dem Körper (erwägen Sie eine Dialyse von Anfang an oder bei Versagen der medikamentösen Therapie).

\section{Modifikationen der kardiopulmonalen} Reanimation

Die folgenden Änderungen der StandardALS-Leitlinien werden bei Vorliegen einer schweren Hyperkaliämie empfohlen:

- Sichern Sie die Hyperkaliämie möglichst durch eine Blutgasanalyse, sofern verfügbar.

- Schützen Sie das Herz: Geben Sie schnell einen Bolus von $10 \mathrm{ml}$ Calciumchlorid $10 \%$ i.v..

- Verschieben Sie Kalium in die Zellen: Geben Sie schnell einen Bolus von Glukose-Insulin (10 Einheiten kurzwirksames Insulin auf $25 \mathrm{~g}$ Glukose i.v.. Überwachen Sie den Blutzuckerspiegel).

- Geben Sie Natriumbikarbonat: $50 \mathrm{mmol}$ i.v. durch schnelle Bolusinjektion, wenn eine schwere Azidose oder ein Nierenversagen vorliegt.

- Entfernen Sie Kalium aus dem Körper: Fassen Sie eine Dialyse ins Auge für einen therapieresistenten hyperkaliämischen Kreislaufstillstand. Mehrere Dialyseverfahren wurden 


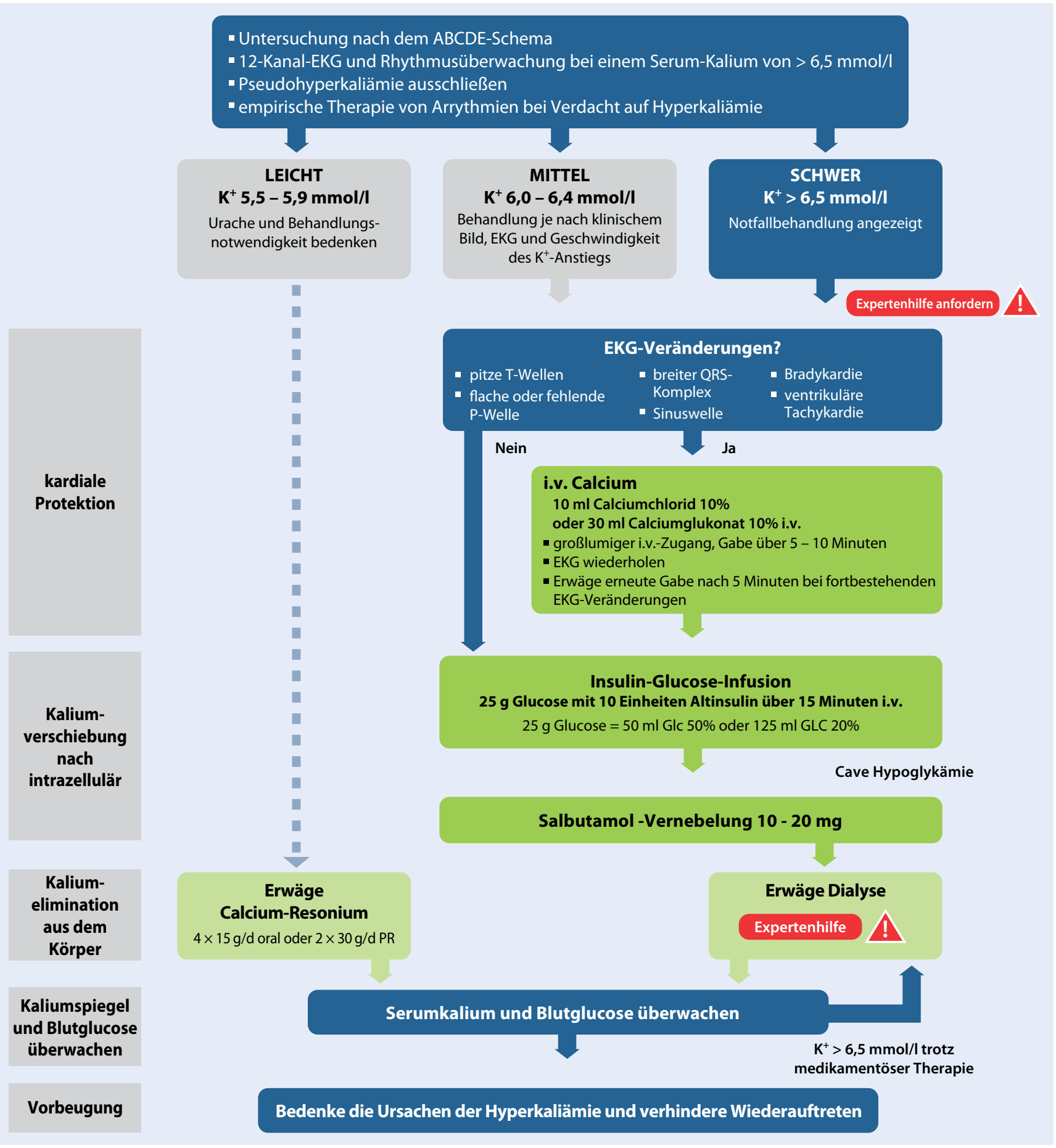

Abb. $1 \Delta$ Notfallbehandlung der Hyperkaliämie (PR per rectum). [Reproduziert mit freundl. Erlaubnis des Renal Association and Resuscitation Council (UK)]

in spezialisierten Zentren sicher und effektiv im Kreislaufstillstand angewendet [25].

- Erwägen Sie den Einsatz eines mechanischen Thoraxkompressionsgeräts, wenn eine länger dauernde Reanimation erforderlich ist.
Indikationen für eine Dialyse Die wesentlichen Indikationen für Patienten mit Hyperkaliämie sind:

- schwere, lebensbedrohliche Hyperkaliämie mit oder ohne EKGVeränderungen oder Arrythmien,

- therapieresistente Hyperkaliämie,

- terminales Nierenversagen,
- oligurisches akutes Nierenversagen (Urinausscheidung < $400 \mathrm{ml}$ pro Tag), - deutlicher Gewebsuntergang, z. B. Rhabdomyolyse.

Spezielle Überlegungen zur Behandlung des Kreislaufstillstands in einer Dialyseeinheit finden sich im Abschnitt „Be- 
sondere Umstände" unter Kreislaufstillstand bei Dialyse.

\section{Hypokaliämie}

Hypokaliämie ist die häufigste Elektrolytstörung in der klinischen Praxis [26]. Sie wird bei bis zu $20 \%$ aller Krankenhauspatienten festgestellt [27]. Hypokaliämie steigert die Inzidenz von Arrhythmien und plötzlichem Herztod („sudden cardiac death", SCD, [28]). Das Risiko steigt noch bei vorbestehender Herzkrankheit und bei Behandlung mit Digoxin.

\section{Definition}

Hypokaliämie ist definiert als Serumkaliumwert $<3,5 \mathrm{mmol} / \mathrm{l}$. Die schwere Hypokaliämie als Serumkalium $<2,5 \mathrm{mmol} / \mathrm{l}$; sie kann symptomatisch sein.

\section{Ursachen}

Die Hauptursachen der Hypokaliämie sind:

- gastrointestinaler Verlust, z. B. durch Durchfall,

- Medikamente, z. B. Diuretika, Abführmittel, Steroide,

- renale Verluste, z. B. tubuläre Störungen, Diabetes insipidus, Dialyse,

- endokrine Störungen, z. B. CushingSyndrom, Hyperaldosteronismus,

- metabolische Alkalose,

- Magnesiummangel,

- geringe Zufuhr mit der Nahrung.

Die Behandlungsstrategien der Hyperkaliämie können auch zu Hypokaliämie führen.

\section{Erkennen der Hypokaliämie}

Schließen Sie eine Hypokaliämie bei jedem Patienten mit einer Arrhythmie oder einem Kreislaufstillstand aus. Bei Dialysepatienten kann die Hypokaliämie am Ende einer Dialysesitzung auftreten oder während einer Peritonealdialyse.

Wenn der Kaliumspiegel fällt, sind vorwiegend Nerven und Muskeln betroffen, wodurch Ermüdung, Schwäche, Beinkrämpfe und Obstipation entstehen.

In schweren Fällen (Serumkalium $<2,5 \mathrm{mmol} / \mathrm{l}$ ) können Rhabdomyolyse, aufsteigende Lähmung und Atemprobleme auftreten.

EKG-Zeichen der Hypokaliämie sind:

- U-Wellen,

- flache T-Wellen,

- ST-Streckenveränderungen,

- Rhythmusstörungen, insbesondere wenn der Patient Digoxin einnimmt,

- Kreislaufstillstand (PEA, Kammerflimmern, pVT, Asystolie).

\section{Behandlung}

Die Behandlung ist abhängig von der Schwere der Hypokaliämie und dem Auftreten von Symptomen und EKG-Veränderungen. Ein schrittweiser Kaliumersatz ist wünschenswert, aber im Notfall ist i.v.-Kalium erforderlich. Maximal werden i.v.-Dosen von $20 \mathrm{mmol} / \mathrm{h}$ empfohlen, aber eine schnellere Infusion (z. B. $2 \mathrm{mmol} / \mathrm{min}$ für $10 \mathrm{~min}$, gefolgt von $10 \mathrm{mmol}$ über 5-10 min) kann bei instabilen Arrhythmien und unmittelbar drohendem Kreislaufstillstand erforderlich sein. Kontinuierliches EKGMonitoring ist während der Kaliuminfusion unverzichtbar, und die Dosis soll durch mehrfache Kaliummessungen titriert werden.

Viele Patienten, die einen Kaliummangel haben, leiden auch unter Magnesiummangel. Magnesium ist wichtig für die Kaliumaufnahme und für die Aufrechterhaltung des intrazellulären Kaliumspiegels, insbesondere im Myokard. Durch Auffüllen der Magnesiumspeicher kann eine Hypokaliämie leichter korrigiert werden, sodass diese Maßnahme bei schwerer Hypokaliämie empfohlen wird.

\section{Störungen des Calcium- und Magnesiumhaushalts}

Die Maßnahmen zur Erkennung und Behandlung von Störungen des Calciumund Magnesiumhaushalts sind in $\bullet$ Tab. 2 zusammengefasst.

\section{Hypo-/Hyperthermie}

\section{Akzidentelle Hypothermie}

\section{Definition}

Jedes Jahr sterben in den USA ca. 1500 Menschen an primärer akzidenteller Hypothermie [33]. Akzidentelle Hypo- thermie ist definiert als unbeabsichtigter Abfall der Körperkerntemperatur $<35^{\circ} \mathrm{C}$. Um die Schwere der Hypothermie an der Unfallstelle abzuschätzen, wird oft das Schweizer Klassifizierungssystem benützt. Es basiert auf klinischen Zeichen, welche grob mit der Kerntemperatur korrelieren:

- Stadium I (milde Hypothermie): Bewusstsein klar, Kältezittern, Kerntemperatur $35-32^{\circ} \mathrm{C}$;

- Stadium II (mäßige Hypothermie): Bewusstsein eingetrübt, kein Kältezittern, Kerntemperatur $32-28^{\circ} \mathrm{C}$;

- Stadium III (schwere Hypothermie): Bewusstlosigkeit, Lebenszeichen vorhanden, Kerntemperatur $28-24^{\circ} \mathrm{C}$;

- Stadium IV: Kreislaufstillstand oder minimaler Kreislauf, keine oder nur minimale Lebenszeichen, Kerntemperatur $<24^{\circ} \mathrm{C}$;

- Stadium V: Tod durch irreversible Hypothermie, Kerntemperatur $<13,7^{\circ} \mathrm{C}[34]$.

\section{Diagnose}

Eine Hypothermie wird diagnostiziert, wenn die Kerntemperatur $<35^{\circ} \mathrm{C}$ beträgt oder (falls eine Temperaturmessung nicht möglich ist) wenn der Patient anamnestisch der Kälte ausgesetzt war oder sich am Stamm kalt anfühlt [33]. In Ländern mit gemäßigtem Klima wird die akzidentelle Hypothermie gelegentlich übersehen. Wenn die Temperaturregulation beeinträchtigt ist, z. B. bei älteren oder sehr jungen Menschen, kann eine akzidentelle Hypothermie schon bei milder Kälteexposition auftreten. Das Risiko einer Hypothermie ist erhöht nach Alkohol- oder Drogenkonsum, bei Erschöpfung, Erkrankung, Verletzung oder Verwahrlosung, v. a. wenn zusätzlich eine Bewusstseinstrübung besteht.

Zur Bestätigung der klinischen Verdachtsdiagnose wird ein Thermometer, das auch niedrige Temperaturen misst, benötigt. Die im unteren Drittel des Oesophagus gemessene Temperatur korreliert gut mit der Temperatur des Herzmuskels. Die mithilfe der Thermistortechnik über dem Trommelfell gemessene (tympanische) Temperatur ist eine zuverlässige Alternative, kann jedoch bei sehr tiefer Umgebungstemperatur, schlechter Isolation 
Tab. 2 Störungen des Calcium- und Magnesiumhaushalts mit klinischer Symptomatik, EKG-Veränderungen, empfohlener Behandlung und assoziierter klinischer Präsentation

\begin{tabular}{|c|c|c|c|c|}
\hline Zustand & Ursachen & Symptome & EKG-Veränderungen & Behandlung \\
\hline $\begin{array}{l}\text { Hyperkalzämie } \\
\text { Calcium } \\
>2,6 \mathrm{mmol} \mathrm{L}^{-1}\end{array}$ & $\begin{array}{l}\text { Primärer oder tertiärer } \\
\text { Hyperparathyreoidismus, } \\
\text { Krebserkrankungen, } \\
\text { Sarkoidose, Medikamente }\end{array}$ & $\begin{array}{l}\text { Verwirrtheit, Schwäche, } \\
\text { abdominelle Schmerzen, } \\
\text { Hypotension, } \\
\text { Arrhythmien, Kreislauf- } \\
\text { stillstand }\end{array}$ & $\begin{array}{l}\text { Verkürztes QT-Intervall, } \\
\text { verlängertes QRS-Intervall, } \\
\text { flache T-Wellen, AV-Block, } \\
\text { Kreislaufstillstand }\end{array}$ & $\begin{array}{l}\text { Substitution von Flüssigkeit i.v., } \\
\text { Furosemid } 1 \text { mg/kgKG i.v., Hydro- } \\
\text { kortison } 200-300 \text { mg i.v., Pamidronsäure } \\
\text { 30-90 mg i.v., Behandlung der Grund- } \\
\text { erkrankung }\end{array}$ \\
\hline $\begin{array}{l}\text { Hypokalzämie } \\
\text { Calcium } \\
<2,1 \mathrm{mmolL}^{-1}\end{array}$ & $\begin{array}{l}\text { Chronische Nieren- } \\
\text { insuffizienz, akute } \\
\text { Pankreatitis, Über- } \\
\text { dosierung von Kalzium- } \\
\text { antagonisten, Toxic-shock- } \\
\text { Syndrom, Rhabdomyolyse, } \\
\text { Tumorlysesyndrom }\end{array}$ & $\begin{array}{l}\text { Parästhesie, Tetanie, } \\
\text { Krämpfe, AV-Block, Kreis- } \\
\text { laufstillstand }\end{array}$ & $\begin{array}{l}\text { Verlängertes QT-Intervall, } \\
\text { Inversion der T-Welle, Herz- } \\
\text { block, Kreislaufstillstand }\end{array}$ & $\begin{array}{l}\text { Calciumchlorid } 10 \% \text { ig, } 10-40 \mathrm{ml} \\
\text { Magnesiumsulfat } 50 \% \text { ig, } 4-8 \mathrm{mmol} \\
\text { (wenn nötig) }\end{array}$ \\
\hline $\begin{array}{l}\text { Hypermagnesiämie } \\
\text { Magnesium } \\
>1,1 \mathrm{mmol} / \mathrm{l}\end{array}$ & $\begin{array}{l}\text { Niereninsuffizienz } \\
\text { iatrogen }\end{array}$ & $\begin{array}{l}\text { Verwirrtheit, Schwäche, } \\
\text { Atemdepression, AV- } \\
\text { Block, Kreislaufstillstand }\end{array}$ & $\begin{array}{l}\text { Verlängertes PR- und QT- } \\
\text { Intervall, spitze T-Welle, AV- } \\
\text { Block, Kreislaufstillstand }\end{array}$ & $\begin{array}{l}\text { Therapie erwägen bei Magnesiumwerten } \\
1,7 \mathrm{mmol} / \mathrm{l} \\
\text { Kalziumchlorid } 10 \% \text { ig, 5-10 ml (wenn } \\
\text { nötig wiederholt) } \\
\text { Beatmung: wenn nötig, forcierte Diurese: } \\
0,9 \% \text { ige NaCl-Lösung mit Furosemid } \\
1 \mathrm{mg} / \mathrm{kgKG} \text { i.v., Hämodialyse }\end{array}$ \\
\hline $\begin{array}{l}\text { Hypomagnesiämie } \\
\text { Magnesium } \\
<0,6 \mathrm{mmol} / \mathrm{l}\end{array}$ & $\begin{array}{l}\text { Gastrointestinaler Ver- } \\
\text { lust, Polyurie, Mangeler- } \\
\text { nährung, Alkoholismus, } \\
\text { Malabsorption }\end{array}$ & $\begin{array}{l}\text { Tremor, Ataxie, } \\
\text { Nystagmus, Krämpfe, } \\
\text { Arrhythmien: Torsade } \\
\text { de pointes, Kreislaufstill- } \\
\text { stand }\end{array}$ & $\begin{array}{l}\text { Verlängertes PR- und } \\
\text { QT-Intervall, ST-Senkung, } \\
\text { Inversion der T-Welle, } \\
\text { abgeflachte P-Wellen, ver- } \\
\text { längerte QRS-Dauer, Torsade } \\
\text { de pointes }\end{array}$ & $\begin{array}{l}\text { Schwer oder symptomatisch: } \\
2 \mathrm{~g} 50 \% \text { iges Magnesiumsulfat ( } 4 \mathrm{ml} \\
=8 \mathrm{mmol} \text { ) i.v. über } 15 \mathrm{~min} \text {, Torsade } \\
\text { de pointes: } 2 \mathrm{~g} 50 \% \text { iges Magnesium- } \\
\text { sulfat ( } 4 \mathrm{ml}=8 \mathrm{mmol} \text { ) i.v. über } 1-2 \mathrm{~min} \text {, } \\
\text { Krämpfe: } 2 \mathrm{~g} 50 \% \text { iges Magnesiumsulfat } \\
\text { (4 } \mathrm{ml}=8 \mathrm{mmol} \text { ) i.v. über } 10 \mathrm{~min}\end{array}$ \\
\hline
\end{tabular}

der Sonde oder bei verlegtem äußerem Gehörgang (Schnee, Wasser) deutlich tiefer sein als die ösophageale Kerntemperatur [35, 36]. Die weit verbreiteten Ohrthermometer, welche auf Infrarottechnik basieren, dichten den Gehörgang nicht genügend $\mathrm{ab}$ und sind nicht für die Messung von tiefen Kerntemperaturen vorgesehen [37].

Während der Wiederbelebung und Aufwärmung im Krankenhaus soll immer die gleiche Messmethode verwendet werden [38, 39]. Deshalb hat die Messung der Blasen- und Rektaltemperatur bei Patienten mit schwerer Hypothermie an Bedeutung verloren.

\section{Entscheidung zum Wieder- belebungsversuch}

Die Abkühlung des menschlichen Körpers reduziert den Sauerstoffverbrauch der Zellen um ca. $6 \%$ pro $1^{\circ} \mathrm{C}$ Abnahme der Kerntemperatur [40]. Bei $28^{\circ} \mathrm{C}$ ist der Sauerstoffverbrauch um $50 \%$, bei $22^{\circ} \mathrm{C}$ sogar um $75 \%$ reduziert. Bei $18^{\circ} \mathrm{C}$ kann das Gehirn einen zehnmal längeren Kreislaufstillstand ertragen als bei $37^{\circ} \mathrm{C}$. Deshalb kann die Hypothermie Herz und
Gehirn im Falle eines Kreislaufstillstands schützen [41], sodass eine vollständige neurologische Erholung auch nach längerem Kreislaufstillstand möglich ist, sofern die schwere Hypothermie vor Auftreten der Asphyxie eingetreten ist.

Seien Sie zurückhaltend mit der Todesfeststellung bei einem hypothermen Patienten, da Kälte allein einen sehr langsamen, fadenförmigen, unregelmäßigen Puls und einen nicht messbaren Blutdruck zur Folge haben kann. Bei einem tief hypothermen Patienten (Stadium IV) können die Lebenszeichen so minimal sein, dass sie leicht übersehen werden. Deshalb sollen Lebenszeichen während mindestens einer Minute gesucht und die elektrische Aktivität des Herzmuskels mittels EKG-Monitor abgeleitet werden. Es wurde berichtet, dass Patienten einen hypothermiebedingten Kreislaufstillstand mit Kerntemperatur von $13,7^{\circ} \mathrm{C}$ [42] und eine Wiederbelebung von 6,5 h [43] ohne neurologische Schäden überlebt haben.

Wenn die Umstände keine kontinuierliche CPR erlauben, kann auch eine intermittierende Herz-Lungen-Wiederbelebung nützlich sein [44]. Kann CPR nicht kontinuierlich durchgeführt werden, soll ein Patient mit Kreislaufstillstand und einer Kerntemperatur von $<28^{\circ} \mathrm{C}$ (oder unbekannter Temperatur) 5 min CPR erhalten, im Wechsel mit $\leq 5$ min Pause. Bei Patienten mit Kerntemperatur $<20^{\circ} \mathrm{C}$ genügen $5 \mathrm{~min} \mathrm{CPR}$ im Wechsel mit $\leq 10$ min Pause [45].

Im präklinischen Umfeld soll auf eine Wiederbelebung hypothermer Patienten nur dann verzichtet werden, wenn der Kreislaufstillstand eindeutig auf eine tödliche Verletzung oder Erkrankung zurückgeführt werden kann, wenn ein prolongierter Atemstillstand vorliegt oder wenn sich der Brustkorb nicht komprimieren lässt [46]. In allen andern Fällen gilt der Grundsatz: "Niemand ist tot, ehe er nicht warm und tot ist." Allerdings muss berücksichtigt werden, dass in abgelegenen Gebieten eine Aufwärmung nicht durchführbar ist. Ziehen Sie im Krankenhaus erfahrene Ärzte hinzu, um aufgrund der klinischen Beurteilung zu entscheiden, wann ein Wiederbelebungsversuch bei einem hypothermen Patienten mit Kreislaufstillstand abgebrochen werden soll. 


\section{Modifikationen der Herz- Lungen-Wiederbelebung bei Hypothermie}

- Verzögern Sie nicht die sorgfältige tracheale Intubation, wenn sie indiziert ist. Die Vorteile der Atemwegssicherung und der adäquaten Sauerstoffversorgung überwiegen gegenüber dem kleinen Risiko, durch die tracheale Intubation ein Kammerflimmern auszulösen [47].

- Suchen Sie bis zu einer Minute nach Lebenszeichen, bevor Sie die Diagnose Kreislaufstillstand stellen. Tasten Sie eine zentrale Arterie, und beurteilen Sie so den Herzrhythmus, falls ein EKG-Monitor nicht verfügbar ist. Die Echokardiographie, eine Dopplerultraschalluntersuchung der Gefäße oder die Nahinfrarotspektroskopie können herangezogen werden, um das Herzminutenvolumen oder den peripheren Blutfluss zu beurteilen [48, 49]. Im Zweifelsfall beginnen Sie sofort mit der Wiederbelebung.

- Die Hypothermie kann zu Steifigkeit des Thorax führen, wodurch die Beatmung und die Thoraxkompression erschwert werden. Erwägen Sie deshalb den Einsatz von mechanischen Thoraxkompressionsgeräten [50].

- Messen Sie die Kerntemperatur, sobald die Herz-Lungen-Wiederbelebung gestartet wurde, und verwenden Sie dazu ein Spezialthermometer das auch niedrige Temperaturen misst.

- Das hypotherme Herz spricht möglicherweise auf herzwirksame Medikamente, Schrittmacherstimulation oder Defibrillation nicht an. Der Medikamentenabbau ist verlangsamt, was zu potenziell toxischen Plasmakonzentrationen führen kann [51]. Die Datenlage zur Wirksamkeit der Medikamente bei schwerer Hypothermie ist beschränkt und basiert hauptsächlich auf Tierstudien. Beispielsweise ist die Wirksamkeit von Amiodaron bei schwerer Hypothermie reduziert [52]. Adrenalin kann den koronaren Blutfluss verbessern, nicht aber die Überlebenschancen $[53,54]$. Vasopressoren erhöhen vielleicht die Chancen einer erfolgreichen Defibrillation, aber bei einer Kerntemperatur $<30^{\circ} \mathrm{C}$ degeneriert der Sinusrhythmus oft wieder zu Kammerflimmern. Da die Defibrillation und Adrenalin das Myokard schädigen können, soll mit der Defibrillation und der Gabe von Adrenalin und andern Medikamenten gewartet werden, bis der Patient eine Kerntemperatur von $\geq 30^{\circ} \mathrm{C}$ erreicht hat. Sobald $30^{\circ} \mathrm{er}-$ reicht sind, können die Dosierungsintervalle für Pharmaka gegenüber denen bei Normothermie verdoppelt werden (z. B. Adrenalin alle 6-10 min). Erst ab Erreichen der Normothermie $\left(\geq 35^{\circ} \mathrm{C}\right)$ sollen die Standardempfehlungen angewendet werden.

\section{Behandlung von Rhythmus- störungen}

Wenn die Kerntemperatur abfällt, geht der meist bradykarde Sinusrhythmus oft in ein Vorhofflimmern über, gefolgt von Kammerflimmern und schließlich Asystolie [55, 56]. Alle Rhythmusstörungen außer Kammerflimmern verschwinden normalerweise spontan, wenn die Kerntemperatur wieder ansteigt und müssen nicht unmittelbar behandelt werden. Im Rahmen einer schweren Hypothermie ist eine Bradykardie physiologisch. Eine Schrittmacherbehandlung ist nicht notwendig, außer die Bradykardie und Beeinträchtigung des Kreislaufs bleibt nach Wiedererwärmung bestehen. Bei welcher Temperatur der erste Defibrillationsversuch unternommen und wie oft ein schwer hypothermer Patient defibrilliert werden soll, ist unklar. Wenn Kammerflimmern festgestellt wird, soll deshalb gemäß Standardempfehlung defibrilliert werden. Wenn das Kammerflimmern nach drei Schocks persistiert, soll auf weitere Versuche verzichtet werden, bis die Kerntemperatur $\geq 30^{\circ} \mathrm{C}$ erreicht hat [57]. Herz-LungenWiederbelebung und Aufwärmung müssen u. U. mehrere Stunden lang durchgeführt werden, um eine erfolgreiche Defibrillation zu ermöglichen.

\section{Verhindern des Wärmeverlusts}

$\mathrm{Zu}$ den allgemeinen Maßnahmen gehören die Rettung aus der kalten Um- gebung, die Verhinderung weiteren Wärmeverlusts und der rasche Transport ins Krankenhaus [58]. Präklinisch soll man einen Patienten mit mäßiger oder schwerer Hypothermie (Stadium $\geq$ II) immobilisieren, sorgfältig mit ihm umgehen, ihn adäquat oxigenieren und monitorisieren (EKG und Kerntemperatur). Der gesamte Körper soll abgetrocknet und isoliert werden [51].

Entfernen Sie nasse Kleider eher durch Aufschneiden als durch Ausziehen, um übermäßiges Bewegen des Körpers zu vermeiden. Die Entfernung von nasser Kleidung scheint für die Verhinderung von Wärmeverlust ähnlich wirksam zu sein wie der Einsatz einer Dampfbarriere [59]. Bewusstseinsklare Opfer einer milden Hypothermie (Stadium I) können sich bewegen, da Bewegung den Körper schneller erwärmt als Kältezittern [60]. Die Patienten kühlen nach der Rettung weiter ab („after drop“), was zu einem lebensbedrohlichen Abfall der Kerntemperatur und dadurch ausgelöstem Kreislaufstillstand auf dem Transport führen kann (Bergungstod). Präklinisch soll auf unnötige Untersuchungen und Behandlungen verzichtet werden, da der weitere Wärmeverlust nur ungenügend verhindert werden kann. Patienten, bei denen das Kältezittern verschwindet (Stadium II-IV), deren Bewusstsein getrübt ist oder die anästhesiert sind, kühlen schneller aus.

\section{Aufwärmung in der Präklinik}

Die Aufwärmung kann passiv, aktiv, extern oder intern erfolgen. Bei Hypothermie Stadium I ist die passive Aufwärmung angemessen, da die Patienten noch Kältezittern haben. Die passive Aufwärmung wird am besten erreicht durch Ganzkörperisolation mittels Wolldecken, Aluminiumfolie, Mütze und eine warme Umgebung (z. B. geheiztes Fahrzeug). Bei Hypothermie Stadium II-IV wird die Anwendung von chemischen Wärmeelementen am Stamm empfohlen. Bei bewusstseinsklaren Patienten, deren Kältezittern noch erhalten ist, verbessert dies den Komfort, beschleunigt die Aufwärmung aber nicht [61]. Bewusstlose Patienten, deren Atemweg nicht gesichert ist, sollen in Seitenlage gebracht und dann zugedeckt werden. Präklinisch 
ist die Aufwärmung mit gewärmten Infusionslösungen oder gewärmten und angefeuchteten Atemgasen nicht durchführbar [51]. Intensive Aufwärmung in der Präklinik soll den Transport ins Krankenhaus, wo aktive Aufwärmtechniken und kontinuierliche Überwachung vorhanden sind, nicht verzögern.

\section{Transport}

Patienten mit Hypothermie Stadium I sollen ins nächstgelegene Krankenhaus transportiert werden. Bei Patienten mit Hypothermie Stadium II-IV, Zeichen der kardialen Instabilität (systolischer Blutdruck <90 mmHg, ventrikuläre Arrhythmie, Kerntemperatur $<28^{\circ} \mathrm{C}$ ) soll der klinische Zustand die Wahl des Zielkrankenhauses bestimmen. Bei jeglichen Zeichen der kardialen Instabilität soll der Patient in ein ECLS-Zentrum („extracorporeal life support") gebracht werden (ECLS umfasst Herz-Lungen-Maschine, extrakorporale Membranoxygenierung oder extrakorporale Lungenassistenz). Diese Klinik muss rechtzeitig kontaktiert werden, um sicherzustellen, dass der Patient dort aufgenommen und behandelt werden kann. Bei Hypothermie Stadium V sollen zunächst Gründe gesucht werden, auf die Wiederbelebung und Aufwärmung zu verzichten [z. B. sichere Todeszeichen, gültige Patientenverfügungen, z. B. DNAR („do not attempt resuscitation“), Gefahr für die Retter, Lawinenverschüttung während $>60$ min, mit Schnee verlegtem Atemweg und Asystolie). Ist keines dieser Zeichen vorhanden, soll mit der Wiederbelebung begonnen, und der Patient muss in ein ECLS-Zentrum transportiert werden.

\section{Aufwärmung im Krankenhaus}

Solange ein Kreislauf vorhanden ist, kann der Patient minimal-invasiv mit Warmluftdecken (z. B. „bair-hugger“) und gewärmten Infusionslösungen aufgewärmt werden. Bei einer Kerntemperatur $<32^{\circ} \mathrm{C}$ und einem Serumkalium $<8 \mathrm{mmol} / \mathrm{l}$ soll eine invasive Aufwärmung mittels ECLS erwogen werden [33]. Meist wurde bisher dazu die Herz-Lungen-Maschine verwendet. Seit einiger Zeit wird jedoch bevorzugt der venoarterielle extrakorporelle Membranoxigenator (VA-ECMO) ein- gesetzt, da diese Geräte rascher verfügbar sind, die Patienten weniger stark antikoaguliert werden müssen und man die Geräte nach erfolgter Aufwärmung auch zur weiteren Kreislaufunterstützung verwenden kann.

Wenn kein ECLS-Zentrum verfügbar ist, kann die Aufwärmung mittels Kombination von externer und interner Erwärmung versucht werden (Warmluftdecken, warme Infusionslösungen, warme Peritoneallavage). Dazu muss jedoch ein spezifisches Behandlungsteam gebildet werden [62].

Die kontinuierliche hämodynamische Überwachung und warme Infusionslösungen sind sehr wichtig. Die Patienten benötigen in der Aufwärmphase wegen der Vasodilatation und der damit verbundenen Erweiterung des intravasalen Raums große Mengen Flüssigkeit. Während und nach der Aufwärmung soll eine Hyperthermie vermieden werden. Sobald der Spontankreislauf wiederhergestellt ist, erfolgt die weitere $\mathrm{Be}$ handlung gemäß den Standards der Postreanimationsversorgung.

\section{Hyperthermie}

\section{Einleitung}

Zur Hyperthermie kommt es, wenn die Thermoregulation des Körpers versagt und die Kerntemperatur über den Bereich ansteigt, der normalerweise durch die Mechanismen der Homöostase gehalten wird. Die Hyperthermie kann exogen durch Umweltbedingungen oder endogen durch erhöhte Wärmeproduktion des Körpers verursacht werden.

Zur umweltbedingten Hyperthermie kommt es, wenn Wärmeenergie, meist in Form von Wärmestrahlung, durch den Körper rascher absorbiert wird, als sie durch die Thermoregulationsmechanismen abgegeben werden kann. Die Hyperthermie umfasst ein Kontinuum von hitzeinduzierten Zuständen, beginnend mit Hitzestress über Hitzeerschöpfung bis hin zum Hitzschlag und schließlich zum Multiorganversagen und Kreislaufstillstand [63].

Die maligne Hyperthermie ist demgegenüber eine seltene, genetisch bedingte Erkrankung der Calciumhomöostase in der Skelettmuskulatur, welche charakterisiert ist durch Muskelkontrakturen und eine lebensbedrohliche hypermetabole Krise, ausgelöst durch Exposition mit halogenierten Inhalationsanästhetika und depolarisierenden Muskelrelaxantien (Succinylcholin, [64, 65]).

\section{Hitzeerschöpfung}

\section{Definition}

Bei der Hitzeerschöpfung handelt es sich um ein nicht lebensbedrohliches Syndrom, bestehend aus Schwäche, Malaise, Übelkeit, Hypotonie und anderen unspezifischen Symptomen, das ausgelöst wird durch Hitzeexposition. Die Thermoregulation ist dabei nicht beeinträchtigt. Die Hitzeerschöpfung wird verursacht durch Wasser- und Elektrolytstörungen infolge Hitzeexposition mit oder ohne körperliche Anstrengung. Selten kann eine schwere Hitzeerschöpfung nach körperlicher Anstrengung zu Rhabdomyolyse, Myoglobinurie, akutem Nierenversagen und disseminierter intravasaler Gerinnung (DIC) führen.

\section{Symptome}

Die Symptome sind oft diskret, und die Patienten bemerken u. U. nicht, dass die Hitze die Ursache ist. Schwäche, Schwindel, Kopfschmerzen, Übelkeit und gelegentlich Erbrechen können die Symptome sein. Synkopen infolge lange Stehens in der Hitze sind häufig und können Herz-Kreislauf-Störungen vortäuschen. Bei der Untersuchung erscheinen die Patienten müde, schwitzen und sind tachykard. Anders als bei Hitzschlag ist das Bewusstsein typischerweise klar. Die Temperatur ist meist normal; falls sie doch erhöht ist, übersteigt sie nie $40^{\circ} \mathrm{C}$.

\section{Diagnose}

Die Diagnose wird klinisch gestellt, wobei andere Ursachen wie z. B. Hypoglykämie, akutes Koronarsyndrom oder Infektionen durch entsprechende Laboruntersuchungen ausgeschlossen werden müssen. 


\section{Behandlung}

\section{Flüssigkeits- und Elektrolytersatz}

Zur Behandlung gehört es, die Patienten in eine kühle Umgebung zu bringen, flach hinzulegen und intravenös kristalloide Flüssigkeit zu verabreichen. Die perorale Flüssigkeitsgabe ist $u$. U. nicht wirksam genug, um Elektrolyte rasch zu ersetzen, kann aber praktischer sein. Die Geschwindigkeit und die Menge der Zufuhr richtet sich nach dem Alter, den Grunderkrankungen und dem klinischen Effekt. Die Verabreichung von 1-2 1 Kristalloide mit einer Geschwindigkeit von $500 \mathrm{ml} / \mathrm{h}$ ist oft adäquat. Eine äußere Kühlung ist meist nicht notwendig und soll nur erwogen werden bei Patienten mit einer Kerntemperatur $\geq 40^{\circ} \mathrm{C}$.

\section{Hitzschlag \\ Definition}

Der Hitzschlag (HS) ist definiert als Hyperthermie mit einer Kerntemperatur $>40^{\circ} \mathrm{C}$, welche begleitet wird durch eine systemische Entzündungsreaktion, Veränderungen des Bewusstseins und Organfunktionsstörungen verschiedener Ausprägung [63]. Es gibt zwei Formen von HS:

1. Der klassische (nicht anstrengungsinduzierte) Hitzschlag (CHS) wird verursacht durch hohe Umgebungstemperaturen und betrifft oft ältere Menschen im Rahmen von Hitzeperioden [66].

2. Der anstrengungsinduzierte Hitzschlag (EHS) wird verursacht durch starke körperliche Anstrengungen bei hohen Außentemperaturen und/oder hoher Luftfeuchtigkeit und betrifft meist junge, gesunde Menschen [67].

Die Mortalität bei Hitzschlag liegt zwischen 10 und 50\% [68].

\section{Prädisponierende Faktoren}

Wegen ihrer Grunderkrankungen, Medikamente, der verminderten Thermoregulation und der eingeschränkten sozialen Unterstützung haben ältere Menschen ein erhöhtes Risiko für hitzeinduzierte Erkrankungen. Daneben gibt es weitere Faktoren: ungenügende Akklimatisation an die Hitze, Dehydratation, Übergewicht, Alkohol, Herz-Kreislauf-Erkrankungen, Haut- und Systemerkrankungen (Psoriasis, Ekzeme, Sklerodermie, Verbrennungen, zystische Fibrose), Hyperthyreose, Phäochoromozytom, Medikamente und Drogen (Anticholinergika, Heroin, Kokain, Amphetamie, Phenothiazine, Sympathomimetika, Calciumkanal- und Beta-Blocker).

\section{Symptome}

Der HS kann sich präsentieren wie ein septischer Schock und durch ähnliche Mechanismen verursacht werden [69]. Eine Fallserie berichtet über 22 HSPatienten, welche mit Multiorganversagen auf eine Intensivstation aufgenommen wurden; 14 davon starben dort [70]. Zu den klinischen Merkmalen gehörten:

- Kerntemperatur $\geq 40^{\circ} \mathrm{C}$,

- heiße, trockene Haut (50\% der Patienten mit EHS zeigten Schwitzen),

- frühe Zeichen und Symptome (z. B. extreme Müdigkeit, Kopfschmerzen, Ohnmachtsanfälle, Rötung des Gesichts, Erbrechen und Durchfälle),

- kardiovaskuläre Störungen (z. B. Rhythmusstörungen und Hypotonie [71]),

- respiratorische Störungen (z. B. „acute respiratory distress syndrome“, ARDS, [72]),

- Störungen des Zentralnervensystems (z. B. Krämpfe und Koma [73]),

- Leber- und Nierenversagen [74],

- Gerinnungsstörungen,

- Rhabdomyolyse [75].

Differenzialdiagnostisch muss der HS gegen andere Störungen, die mit einer erhöhten Temperatur einhergehen, abgegrenzt werden. Dazu gehören Medikamentenintoxikationen (Serotoninsyndrom, malignes neuroleptisches Syndrom), Entzugssyndrome, Sepsis, Infektionen des Zentralnervensystems und endokrine Störungen (z. B. thyreotoxische Krise, Phäochromozytom).

\section{Behandlung}

Im Vordergrund stehen unterstützende Maßnahmen und die rasche Kühlung des Patienten [76-78]. Wenn möglich, soll mit der Kühlung bereits präklinisch begonnen werden. Das Ziel ist, die Kern- temperatur so rasch wie möglich auf $39^{\circ} \mathrm{C}$ zu senken. Patienten mit schwerem HS müssen auf einer Intensivstation behandelt werden. Große Mengen Flüssigkeit und die Korrektur von Elektrolytstörungen können notwendig sein (s. auch „Hypo-/Hyperkaliämie und andere Elektrolytstörungen“).

\section{Kühltechniken}

Verschiedene Techniken wurden beschrieben, aber nur wenige Studien haben untersucht, welches die optimale Methode ist. Einfache Maßnahmen umfassen das Zuführen von kühlen Getränken, das Befächern und das Besprühen des ausgezogenen Patienten mit lauwarmem Wasser. Das Auflegen von Eisbeuteln auf gut durchblutete Körperregionen (Axilla, Leiste, Nacken) kann ebenfalls nützlich sein. Die Kühlung der Oberfläche löst allerdings möglicherweise Kältezittern aus. Bewusstseinsklare, kooperative und stabile Patienten können zudem in ein kaltes Wasserbad gebracht werden, wobei dies eine periphere Vasokonstriktion und eine Umverteilung des Blutflusses weg von der Peripherie und damit eine verminderte Verteilung der Hitze verursachen kann [79]. Bei sehr kranken Patienten ist diese Methode nicht praktikabel.

Weiter können auch dieselben Techniken eingesetzt werden, die beim gezielten Temperaturmanagement nach Kreislaufstillstand benützt werden (s. auch „Nachsorge nach erfolgreicher Reanimation“, [80]). Die i.v.-Gabe von kalten Flüssigkeiten, Spülungen des Magens, der Bauchhöhle [81], der Pleurahöhle oder der Blase mit kaltem Wasser senkt die Kerntemperatur. Intravaskuläre Kühlungstechniken umfassen neben der i.v.-Gabe von kalten Flüssigkeiten [82] den Einsatz von intravasalen Kühlungskathetern $[83,84]$ und extrakorporalem Kreislauf [85], wie z. B. die kontinuierliche venovenöse Hämofiltration oder die Herz-Lungen-Maschine.

\section{Medikamentöse Therapie}

Es gibt keine spezifischen Medikamente zur Senkung der Kerntemperatur bei HS. Auch dass Antipyretika [z. B. nichtsteroidale Antiphlogistika (NSAID) oder Paracetamol] bei HS wirksam sind, ist 
nicht erwiesen. Diazepam kann zur Behandlung von Krämpfen eingesetzt werden und die Kühlung erleichtern [86]. Dantrolen hatte in mehreren Studien keinen Effekt [87-89].

\section{Maligne Hyperthermie}

Die maligne Hyperthermie ist eine lebensbedrohliche, genetisch bedingte Überempfindlichkeit der Skelettmuskulatur auf halogenierte Inhalationsanästhetika und depolarisierende Muskelrelaxantien (Succinylcholin), welche während oder nach Allgemeinanästhesie auftritt [90]. Stoppen Sie umgehend die Zufuhr der auslösenden Substanzen, geben Sie Sauerstoff, und korrigieren Sie Azidose und Elektrolytstörungen. Beginnen Sie mit der aktiven Kühlung, und verabreichen Sie Dantrolen (s. „Hypo-/Hyperthermie“, [91]).

Drogen [z. B. 3,4-Methylenedioxym ethamphetamine (MDMA, „Ecstasy“) und Amphetamine] können der Hyperthermie ähnliche Zustände verursachen, und der Einsatz von Dantrolen kann hier ebenfalls nützlich sein [92].

\section{Modifikationen der Herz- Lungen-Wiederbelebung bei Hyperthermie}

Es gibt keine spezifischen Studien zum Kreislaufstillstand bei Hyperthermie. Folgen Sie deshalb den Standardleitlinien und kühlen Sie den Patienten weiter, wenn ein Kreislaufstillstand eintritt. Benützen Sie die gleichen Kühltechniken wie beim gezielten Temperaturmanagement in der Nachsorge nach erfolgreicher Reanimation (s. dort, [80]). Defibrillationsversuche werden mit den Standardenergien durchgeführt. Tierexperimente deuten darauf hin, dass die Prognose bei hyperthermem verglichen mit normothermem Kreislaufstillstand sehr schlecht ist $[93,94]$. Pro Grad Körpertemperatur $>37^{\circ} \mathrm{C}$ erhöht sich das Risiko eines ungünstigen neurologischen Behandlungsresultats um den Faktor 2,26 („Odds Ratio“, [95]).

\section{Hypovolämie}

\section{Einleitung}

Die Hypovolämie gehört zu den potenziell behandelbaren Ursachen des
Kreislaufstillstands und beruht meist auf einem reduzieren intravasalen Volumen (z. B. infolge Blutung). Eine relative Hypovolämie kann aber auch bei massiver Vasodilatation im Rahmen einer anaphylaktischen Reaktion oder einer Sepsis auftreten.

Die Hypovolämie infolge mediatorinduzierter Vasodilatation und erhöhter Gefäßpermeabilität gehört zu den wichtigsten Ursachen des Kreislaufstillstands bei Anaphylaxie [96]. Die Hypovolämie infolge Blutverlusts gehört $\mathrm{zu}$ den wichtigsten Todesursachen beim traumabedingten Kreislaufstillstand [97]. Ein äußerer Blutverlust ist meist offensichtlich (äußere Blutung bei Trauma, Hämatemesis, Hämoptyse), während ein innerer Blutverlust (z. B. gastrointestinale Blutung ohne Hämatemesis, rupturiertes Aortenaneurysma) schwieriger zu diagnostizieren ist. Patienten nach größeren Operationen haben ein erhöhtes Risiko der Hypovolämie infolge Nachblutung und müssen entsprechend überwacht werden (s. „Perioperativer Kreislaufstillstand").

Beginnen Sie, abhängig von der vermuteten Ursache, die Volumentherapie so rasch wie möglich mit gewärmten Kristalloiden oder Blutprodukten, um das intravasale Volumen wiederherzustellen. Leiten Sie gleichzeitig Maßnahmen zur Kontrolle der Blutung ein, z. B. chirurgische Blutstillung, Endoskopie oder endovaskuläre Techniken [98] oder behandeln Sie die Ursache (z. B. anaphylaktischer Schock). In der Anfangsphase können Sie jegliches Kristalloid benützen, welches gerade verfügbar ist. Die Ultraschalluntersuchung ist ein hilfreiches diagnostisches Instrument beim hypovolämen Kreislaufstillstand, welches auch während der Herz-Lungen-Wiederbelebung (z. B. während der Rhythmuskontrolle oder der Beatmung, aber ohne die Herzdruckmassage zu unterbrechen) eingesetzt werden kann (und soll), sofern eine in Ultraschall ausgebildete Person zur Verfügung steht.

Die Behandlung des Kreislaufstillstands und der Peri-arrest-Situationen bei Hypovolämie und Anaphylaxie unterscheiden sich und werden deshalb in separaten Abschnitten behandelt.

\section{Anaphylaxie}

\section{Definition}

Für die Notfallbehandlung ist eine präzise Definition der Anaphylaxie nicht wichtig [99]. Der Nomenklaturausschuss der Europäischen Akademie für Allergologie und klinische Immunologie (EACCI) hat folgende Definition vorgeschlagen [100]: Die Anaphylaxie ist eine schwere, lebensbedrohliche, generalisierte oder systemische Reaktion. Diese ist charakterisiert durch rasch auftretende Störungen von Luftweg und/ oder Atmung und/oder Kreislauf, welche meist begleitet werden durch Veränderungen an Haut und Schleimhäuten $[1,96,101,102]$.

\section{Epidemiologie}

Die Anaphylaxie ist häufig, sie betrifft im Laufe des Lebens ca. eine von 300 Personen der europäischen Bevölkerung. Die Inzidenz beträgt zwischen 1,5 und 7,9 pro 100.000 Einwohner und Jahr. Eine Anaphylaxie kann durch eine Vielzahl von Substanzen ausgelöst werden, am häufigsten durch Nahrungsmittel, Medikamente, Insektenstiche und Latex [103]. Nahrungsmittel sind die häufigsten Ursachen bei Kindern, Medikamente die häufigsten bei Erwachsenen [104]. Nahezu alle Nahrungsmittel oder Medikamente können Reaktionen auslösen, wobei die meisten auf spezielle Lebensmittel (z. B. Nüsse) oder Medikamente (Muskelrelaxantien, Antibiotika, nicht-steroidale Entzündungshemmer und Aspirin) zurückzuführen sind [105].

Eine beträchtliche Zahl von anaphylaktischen Reaktionen sind idiopathisch. In Großbritannien wurden zwischen 1992 und 2012 die meisten anaphylaxiebedingten Klinikeinweisungen und Todesfälle im Zusammenhang mit Medikamenten oder Insektenstichen in der Altersgruppe der über 60-Jährigen beobachtet. Demgegenüber waren nahrungsmittelinduzierte Anaphylaxien am häufigsten bei jungen Menschen mit einem Häufigkeitsgipfel der tödlichen Nahrungsmittelreaktionen in der Altersgruppe zwischen 10 und 30 Jahren [106].

Die Prognose der Anaphylaxie ist mit einer Letalität von $<1 \%$ in den 
meisten populationsbasierten Studien gut. Die Daten der Europäischen Anaphylaxie-Datenbank zeigen, dass in nur $2 \%$ von 3333 Fällen ein Kreislaufstillstand auftrat [107]. Bei Patienten, welche auf eine Intensivstation eingewiesen werden mussten, betrug die Überlebensrate bei Krankenhausentlassung $>90 \%$. In den Jahren 2005 bis 2009 kamen in Großbritannien 81 Kinder und 1269 Erwachsene wegen Anaphylaxie auf Intensivstationen. Die Überlebensrate bei Klinikaustritt betrug bei Kindern 95\% und bei Erwachsenen $92 \%$ [108].

Eine erhöhte Letalität besteht hingegen bei Patienten mit vorbestehendem Asthma, besonders wenn dieses schwer oder schlecht kontrolliert und die Behandlung verzögert ist $[109,110]$. Bei einer fatalen Anaphylaxie tritt der Tod häufig sehr rasch nach Kontakt mit dem Auslöser ein. Fallserien zeigten, dass bei fatalen Nahrungsmittelreaktionen der Atemstillstand typischerweise nach 3035 min eintrat, der Kollaps nach Insektenstich bereits nach 10-15 min und Todesfälle nach i.v.-Medikamentengabe meist innerhalb von $5 \mathrm{~min}$. Sechs Stunden nach Kontakt mit dem Auslöser wurden hingegen nie Todesfälle beobachtet [101, 111].

\section{Diagnose der Anaphylaxie}

Die Anaphylaxie ist die wahrscheinlichste Diagnose, wenn ein Patient nach Exposition mit einem Trigger (Allergen) plötzlich (meist innerhalb von Minuten) erkrankt mit rasch sich entwickelnden, lebensbedrohlichen Störungen der Luftwege, der Atmung und des Kreislaufs, meist verbunden mit Veränderungen der Haut und der Schleimhäute. Die Reaktion ist häufig unerwartet.

Die Task-Force Anaphylaxie der EACCI stellt fest, dass eine Anaphylaxie mit hoher Wahrscheinlichkeit vorliegt, wenn eines der folgenden drei Kriterien erfüllt ist $[96,112]$ :

1. Akuter Beginn der Erkrankung (innerhalb von Minuten bis Stunden) mit Beteiligung der Haut, Schleimhäute oder beidem (generalisierte Urticaria, Pruritus, Rötung, Schwellung der Lippen, Zunge und Uvula), kombiniert mit mindestens einem der folgenden Merkmale: a. Atemprobleme, z. B. Dyspnoe, Giemen, spastisches Atemgeräusch, Stridor, reduzierter „peak expiratory flow“ (PEF), Hypoxämie,

b. Blutdruckabfall oder Symptome infolge verminderter Organperfusion, z. B. Synkope, Kollaps, Urinabgang.

2. Zwei oder mehrere der folgenden Zeichen, welche kurz (innerhalb von Minuten bis Stunden) nach Exposition mit einer Substanz auftreten, auf die der vorliegende Patient wahrscheinlich allergisch ist:

a. Veränderungen von Haut und Schleimhäuten, z. B. generalisierte Urticaria, Pruritus, Rötung, Schwellung von Lippen, Zunge und Uvula,

b. Atemprobleme, z. B. Dyspnoe, Giemen, spastisches Atemgeräusch, Stridor, reduzierter PEF, Hypoxämie,

c. Blutdruckabfall oder assoziierte Symptome, z. B. Kollaps (Hypotonie), Synkope, Urinabgang,

d. persistierende gastrointestinale Symptome, z. B. krampfartige Bauchschmerzen, Erbrechen.

3. Blutdruckabfall kurz (innerhalb von Minuten bis Stunden), der mit einer Substanz auftritt, auf die der vorliegende Patient bekannterweise allergisch ist:

a. Säuglinge und Kinder: systolischer Blutdruck $(<70 \mathrm{mmHg}$ von 1 Monat bis $1 \mathrm{Jahr} ;<70 \mathrm{mmHg}$ $+(2 \times$ Alter $)$ zwischen 1 und 10 Jahren; $<90$ mmHg zwischen 11 und 17 Jahren) oder Abfall des systolischen Blutdruckes $>30 \%$ vom Ausgangswert dieser Person,

b. Erwachsene: systolischer Blutdruck $<90$ mmHg oder Abfall des systolischen Blutdruckes $>30 \%$ vom Ausgangswert dieser Person.

\section{Behandlung}

Es gibt nur wenige Daten zur spezifischen Behandlung von Anaphylaxie [113] Allgemein wird das systematische Vorgehen nach dem ABCDE-Schema empfohlen, um sie zu erkennen und zu behandeln, wobei die sofortige intramuskuläre (i.m.) Verabreichung von Adrenalin betont wird (- Abb. 2). Lebensbedrohliche Probleme sollen sofort nach ihrer Diagnose behandelt werden. Die grundlegenden Be- handlungsprinzipien sind für alle Altersgruppen gleich. Alle Patienten, bei denen eine Anaphylaxie vermutet wird, müssen sofort am Monitor überwacht werden (z. B. im Rettungswagen oder auf der Notfallstation). Dazu gehören auf jeden Fall Pulsoximetrie, nicht-invasive Blutdruckmessung und ein 3-Kanal-EKG.

\section{Lagerung des Patienten}

Patienten mit Anaphylaxie können sich rasch verschlechtern, bis hin zum Kreislaufstillstand, wenn man sie aufsetzt oder stehen lässt [114]. Alle Patienten sollen deshalb in eine bequeme Position gebracht werden. Patienten mit einem Problem der Luftwege oder der Atmung werden es bevorzugen zu sitzen, da dies die Atmung erleichtert. Flachlagerung mit oder ohne Anheben der Beine ist hingegen hilfreich bei Patienten mit niedrigem Blutdruck.

\section{Entfernen des Triggers (wenn möglich)} Jegliches Medikament, welches als Ursache infrage kommt, muss umgehend gestoppt werden. Nach einem Wespenoder Bienenstich soll der Stachel sofort entfernt werden. Die Methode der Entfernung ist dabei weniger wichtig, als dass es rasch geschieht [115]. Allerdings soll die Behandlung nicht verzögert werden, wenn der Auslöser nicht entfernt werden kann.

Kreislaufstillstand infolge Anaphylaxie Starten Sie sofort die Herz-LungenWiederbelebung gemäß gültigen Richtlinien. Die Reanimation kann längere Zeit in Anspruch nehmen. Die Ersthelfer sollen sicherstellen, dass rechtzeitig professionelle Hilfe (ALS) angefordert wird, da diese von großer Bedeutung ist.

\section{Atemwegsobstruktion}

Die Anaphylaxie kann Schwellungen und Behinderungen der Luftwege verursachen, dies erschwert Maßnahmen zur Atemwegssicherung und Beatmung (z. B. die Beutel-Masken-Beatmung, tracheale Intubation oder Koniotomie). Die tracheale Intubation soll deshalb frühzeitig erwogen werden, bevor sie durch die Schwellung der Luftwege erschwert wird. Expertenrat soll frühzeitig angefordert werden. 


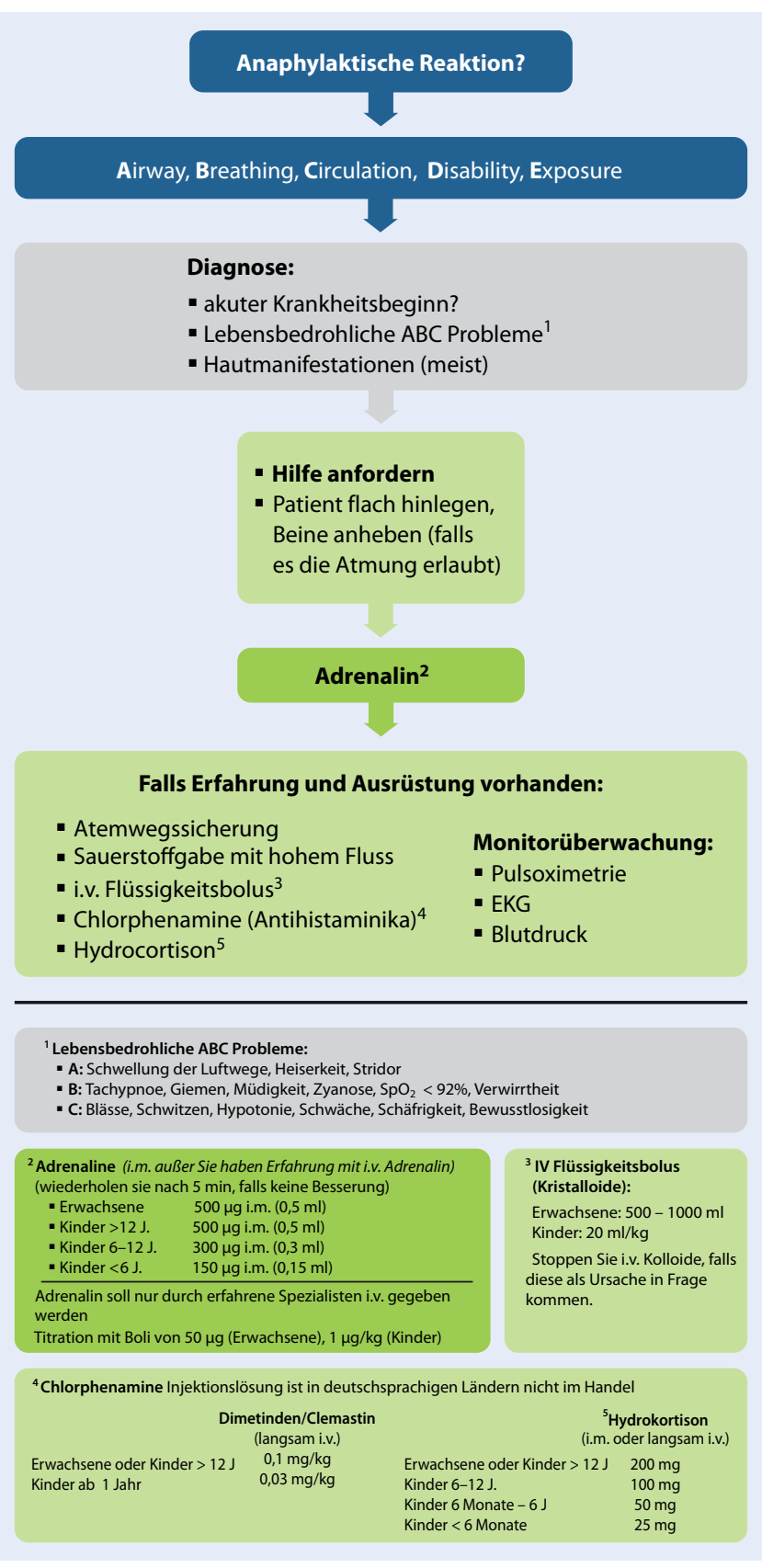

Zeichen einer systemischen allergischen Reaktion, muss der Patient sorgfältig überwacht und symptomatisch nach dem ABCDE-Schema behandelt werden.

\section{I.m.-Gabe von Adrenalin}

Die intramuskuläre (i.m.) Gabe von Adrenalin ist für die meisten Anwender der sicherste und schnellste Applikationsweg zur Behandlung der Anaphylaxie. Der Patient soll so rasch wie möglich am Monitor überwacht werden (Blutdruck, EKG, Pulsoximetrie), damit die Wirkung der Adrenalingabe festgestellt werden kann. Der i.m.-Applikationsweg hat mehrere Vorteile:

- größere therapeutische Sicherheit,

- Anwendung auch ohne i.v.-Zugang möglich,

- einfachere Erlernbarkeit der

Methode,

- Patienten mit bekannten Allergien können sich Adrenalin selber i.m. verabreichen.

Der beste Applikationsort ist die anterolaterale Seite des mittleren Drittels des Oberschenkels. Die Injektionsnadel soll lang genug gewählt werden, sodass das Adrenalin in den Muskel injiziert werden kann [120]. Wegen der schlechteren Wirksamkeit der subkutanen oder inhalativen Anwendung ist der i.m.-Applikationsweg zu bevorzugen [121-123].

\section{Abb. $2<$ Ana-} phylaxiebehandlungsalgorithmus [101]. (Reproduziert mit freundl. Erlaubnis von Elsevier Ireland Ltd)

\section{Adrenalin (Behandlung der ersten Wahl)}

Adrenalin ist das wichtigste Medikament zur Behandlung der Anaphylaxie [116, 117]. Obwohl es keine randomisierten kontrollierten Studien gibt, ist Adrenalin eine logische und erprobte Behandlungsmethode von Bronchospasmus und Kreislaufkollaps [118]. Als Alpha-Rezeptoragonist wirkt es der Vasodilatation entgegen und reduziert das Ödem. Seine Beta-Rezeptoraktivität erweitert die Bronchien, wirkt positiv inotrop und hemmt die Freisetzung von Histamin und Leukotrienen. Die Aktivierung von $\beta_{2}$-adrenergen Rezeptoren auf der Mastzelloberfläche hemmt deren Aktivierung, und die frühe Gabe von Adrenalin vermindert die Schwere einer IgE-vermittelten allergischen Reaktion. Adrenalin ist am wirksamsten, wenn es früh nach Beginn der Reaktion gegeben wird, und Nebenwirkungen sind extrem selten bei korrekter i.m.-Dosierung [119].

Geben Sie allen Patienten mit lebensbedrohlichen Symptomen und Zeichen Adrenalin. Liegen keine lebensbedrohlichen Symptome vor, sondern andere

\section{Dosis für die i.m.-Adrenalingabe}

Es gibt nur wenig Evidenz zur richtigen Dosierung. Die EAACI empfiehlt Adrenalin $(1 \mathrm{mg} / \mathrm{ml})$ i.m. in einer Dosis von $0,01 \mathrm{ml} / \mathrm{kg}$ Körpergewicht (KG), bis $\mathrm{zu}$ einer maximalen Dosis von $0,5 \mathrm{mg}$ [96].

Die folgenden Dosierungen orientieren sich an der Sicherheit und Praktikabilität in Notfallsituationen (die äquivalenten Volumina einer Adrenalinlösung 1:1000 oder $1 \mathrm{mg} / \mathrm{ml}$ sind in Klammern angegeben):

- > 12 Jahre und Erwachsene $500 \mathrm{mcg}$ i.m. $(0,5 \mathrm{ml})$,

- >6 bis 12 Jahre $300 \mathrm{mcg}$ i.m. $(0,3 \mathrm{ml})$,

- >6 Monate bis 6 Jahre $150 \mathrm{mcg}$ i.m. $(0,15 \mathrm{ml})$,

- <6 Monate $150 \mathrm{mcg}$ i.m. (0,15 ml). 
Adrenalin ist am wirksamsten, wenn es frühzeitig nach Beginn der Reaktion gegeben wird, und Nebenwirkungen sind bei korrekter i.m.-Dosierung extrem selten [119].

\section{I.v.-Adrenalingabe (nur für erfahrene Anwender)}

Das Risiko von gefährlichen Nebenwirkungen durch unsachgemäße Dosierung oder Fehldiagnose einer Anaphylaxie ist bei der intravenösen (i.v.) Adrenalingabe viel größer [124]. I.v.-Adrenalin soll deshalb nur durch Erfahrene und im Umgang mit Vasopressoren Geübte eingesetzt werden (z. B. Anästhesisten, Notfallund Intensivmediziner). Bei Patienten mit Spontankreislauf kann i.v.-Adrenalin lebensbedrohliche Hypertensionen, Tachykardien, Rhythmusstörungen und Myokardischämien verursachen. Wenn kein i.v.-Zugang vorhanden ist oder nicht rasch genug gelegt werden kann, soll der i.m.-Applikationsweg gewählt werden. Patienten, welche i.v.-Adrenalin erhalten, müssen am Monitor überwacht werden (mindestens kontinuierlich EKG, Pulsoximetrie und häufige nicht-invasive Blutdruckmessung). Patienten, die wiederholte i.m.-Dosen benötigen (d. h. nicht rasch genug ansprechen), profitieren von i.v.-Adrenalingabe. Bei diesen Patienten ist aber frühzeitige professionelle Betreuung entscheidend.

\section{Dosis für die i.v.-Adrenalingabe (nur für} erfahrene Anwender)

- Erwachsene: Titrieren Sie i.v.Adrenalin in 50-mcg-Boli nach Wirkung. Sind wiederholte Dosen nötig, empfiehlt sich die kontinuierliche Gabe mit Perfusor oder als Infusion $[125,126]$.

- Kinder: I.m.-Adrenalin ist bei Kindern mit Anaphylaxie der bevorzugte Applikationsweg. Die i.v.Gabe soll nur bei entsprechendem Monitoring und durch erfahrene Anwender durchgeführt werden (z. B. Kinderanästhesisten, Kindernotfallmediziner oder Kinderintensivmediziner) und sofern ein i.v.-Zugang rasch genug verfügbar ist. Es gibt keine Daten, auf denen eine Dosisempfehlung basieren könnte.
Die Dosierung erfolgt deshalb nach klinischer Wirkung. Ein Kind kann bereits auf eine Dosis von $1 \mathrm{mcg} / \mathrm{kg}$ reagieren. Diese bedarf einer sorgfältigen Verdünnung und Überprüfung, damit Dosierungsfehler vermieden werden.

Dosis für die i.v./i.o.-Adrenalingabe (nur bei Kreislaufstillstand)

Bei Kreislaufstillstand und vermuteter Anaphylaxie soll mit üblichen Dosen von i.v. oder intraossärem (i.o.) Adrenalin behandelt werden. Falls dies nicht gelingt, kann bei drohendem oder soeben eingetretenem Kreislaufstillstand i.m.Adrenalin in Betracht gezogen werden.

\section{Sauerstoff (sobald verfügbar)}

$\mathrm{Zu}$ Beginn bieten Sie dem Patienten die höchst mögliche Sauerstoffkonzentration mittels Maske mit Reservoir und hohem Fluss (> 10 1/min) an [127]. Wenn der Patient intubiert ist, beatmen Sie ihn mit hohen Sauerstoffkonzentrationen mit dem Beutel.

\section{Flüssigkeit (sobald verfügbar)}

Neben der Vasodilatation kommt es bei der Anaphylaxie wegen der erhöhten Durchlässigkeit der Kapillaren zu großen Volumenverlusten. Beginnen Sie mit der Gabe von i.v.-Flüssigkeit, sobald ein i.v.-Zugang gelegt ist. Bei Kindern sollen $20 \mathrm{ml} / \mathrm{kg}$, bei Erwachsenen ein Bolus von 500-1000 $\mathrm{ml}$ rasch infundiert werden, wobei die Wirkung überwacht werden muss. Bei Bedarf können weitere Boli gegeben werden. Unter Umständen werden große Mengen Flüssigkeit benötigt. Es gibt keine Evidenz, wonach Kolloide besser wirken als Kristalloide. Kolloide müssen jedoch als Ursache in Betracht gezogen werden, wenn bei einem Patienten mit laufender Kolloidinfusion eine Anaphylaxie auftritt. Die Kolloidinfusion muss in diesem Fall sofort gestoppt werden.

Sollte der i.v.-Zugang nicht rasch genug gelingen oder unmöglich sein, kann der i.o.-Applikationsweg sowohl für Flüssigkeiten als auch für Medikamente eingesetzt werden. Allerdings soll die i.m.-Gabe von Adrenalin nicht verzögert werden, weil ein i.o.-Zugang gelegt wird.
Antihistaminika (nach der Erstversorgung)

Antihistaminika gehören zu den Medikamenten der zweiten Reihe bei Anaphylaxie. Es gibt nur wenig Evidenz, die deren Anwendung stützt, aber es bestehen logische Gründe dafür [128]. $\mathrm{H}_{1}$-Antihistaminika helfen, der histamininduzierten Vasodilatation, der Bronchokonstriktion und besonders den Hautsymptomen entgegenzuwirken. Es gibt wenig Evidenz für den routinemäßigen Einsatz von $\mathrm{H}_{2}$-Antihistamika (z. B. Ranitidine, Cimetidine) in der Initialbehandlung der Anaphylaxie.

\section{Kortikosteroide (nach der Erstver- sorgung)}

Kortikosteroide können langwierige Reaktionen verkürzen oder verhindern, wobei die Evidenz dafür begrenzt ist [129]. Besonders Asthmatiker profitieren von der frühzeitigen Behandlung mit Kortikosteroiden. Dies gilt sowohl für Erwachsene als auch für Kinder, wobei es kaum Daten zur optimalen Dosierung gibt.

\section{Andere Medikamente \\ Bronchodilatatoren}

Die Symptome und Zeichen der schweren Anaphylaxie und des lebensbedrohlichen Asthmaanfalls können die gleichen sein. Erwägen Sie deshalb auch eine zusätzliche bronchospasmolytische Behandlung mittels Salbutamol (inhalativ oder i.v.), Ipratropium (inhalativ), Aminophyllin (i.v.) oder Magnesium (i.v.; s. „Asthma“). I.v.-Magnesium wirkt allerdings auch als Vasodilatator und kann deshalb eine Hypotension verschlimmern.

\section{Medikamente mit kardiovaskulärer Wirkung}

Adrenalin bleibt der Vasopressor erster Wahl zur Behandlung der Anaphylaxie. Tierexperimentelle Arbeiten und Fallberichte beschreiben die Wirksamkeit anderer Vasopressoren und Inotropika (Noradrenalin, Vasopressin, Terlipressin, Metaraminol, Methocamine und Glucagon) für den Fall, dass Adrenalin und i.v.-Volumengabe nicht den gewünschten Effekt haben sollten [130-142]. Diese Medikamente dürfen allerdings nur unter spezieller Überwachung (z. B. 
auf der Intensivstation) und durch entsprechend erfahrenes Personal eingesetzt werden. Glucagon kann nützlich sein zur Behandlung einer Anaphylaxie bei Patienten, welche unter Beta-Rezeptorenblockertherapie stehen [143]. In gewissen Fallberichten wird der Einsatz einer HerzLungen-Maschine [144, 145] oder von mechanischen Thoraxkompressionsgeräten vorgeschlagen [146].

\section{Abklärung}

Grundsätzlich sollen die bei medizinischen Notfallsituationen üblichen Untersuchungen durchgeführt werden: z. B. 12-Ableitungs-EKG, Röntgenthorax, Bestimmung von Harnstoff und Elektrolyten und arterielle Blutgasanalyse etc.

\section{Mastzelltryptase}

Der spezifische Test, um die Diagnose der Anaphylaxie zu bestätigen, ist die Bestimmung der Mastzelltryptase. Die Tryptase ist das wichtigste Protein, welches bei der Mastzelldegranulation freigesetzt wird. Deshalb führt die Anaphylaxie zu stark erhöhten Tryptasekonzentrationen im Blut, meist beginnend $30 \mathrm{~min}$ nach Auftreten der Symptome. Das Maximum wird typischerweise 1-2 h nach Symptombeginn erreicht [147]. Die Halbwertzeit der Tryptase ist kurz (ca. 2 h), sodass sich die Konzentrationen innerhalb von 6-8 h wieder normalisieren. Der Zeitpunkt der Blutentnahmen ist deshalb entscheidend. Der Beginn der Anaphylaxie ist gleichbedeutend mit dem Zeitpunkt, zu dem die ersten Symptome festgestellt wurden.

a. Mindestens eine Blutentnahme soll

1-2 h nach Symptombeginn erfolgen.

b. Besser sind jedoch drei Blutproben zu definierten Zeiten:

- die erste so früh wie möglich nach Beginn der Reanimation (ohne diese zu verzögern),

- die zweite 1-2 h nach Beginn der Symptome,

- die dritte entweder $24 \mathrm{~h}$ danach oder in der Nachbehandlung (z. B. anlässlich einer Nachkontrolle in einer Allergieklinik) zur Bestimmung der individuellen Basiskonzentration.
Die Entnahme von mehreren Proben bedeutet bessere Sensitivität und Spezifität als eine einzelne Bestimmung im Hinblick auf die Diagnose einer Anaphylaxie [148].

\section{Entlassung und Nachbehandlung}

Patienten, bei denen eine Anaphylaxie vermutet wird (d. h. mit Atemwegs-, Atmungs- oder Kreislaufproblemen; „airway-breathing-circulation“, ABC) sollen in einer Klinik behandelt und überwacht werden, die die Möglichkeiten zur Behandlung von lebensbedrohlichen ABC-Problemen hat. Wegen des Risikos biphasischer Reaktionen sollen Patienten, welche gut auf die initiale Behandlung ansprechen, über die Möglichkeit eines Rückfalls aufgeklärt oder u. U. zur Überwachung stationär aufgenommen werden. Die exakte Inzidenz biphasischer Reaktionen ist nicht bekannt. Obwohl einzelne Studien von einer Inzidenz von 1-20\% ausgehen, ist nicht klar, ob all diese Patienten wirklich eine Anaphylaxie hatten oder ob die initiale Behandlung angemessen war [149]. Es gibt keine zuverlässige Methode zur Vorhersage, wer eine biphasische Reaktion erleiden wird. Deshalb soll der Entscheid über Entlassung oder stationäre Aufnahme durch einen erfahrenen Kliniker getroffen werden.

Vor Entlassung aus dem Krankenhaus müssen alle Patienten

- von einem Allergologen gesehen werden und einen individuellen Behandlungsplan bekommen,

- klare Anweisungen erhalten, ins Krankenhaus zurückzukehren, sollten die Symptome wieder auftreten,

- bezüglich der Notwendigkeit der Abgabe einer Adrenalinfertigspritze evaluiert werden oder - falls diese verbraucht wurde - einen Ersatz bekommen [150-152], und es muss sichergestellt werden, dass die nötige Anleitung zu deren Anwendung gegeben wurde,

- über die Notwendigkeit und den Ablauf der Weiterbetreuung informiert werden. Der behandelnde Hausarzt soll benachrichtigt werden.

Die Patienten müssen Informationen über das auslösende Allergen erhalten (sofern es identifiziert werden konnte), und sie müssen erfahren, wie sie es vermeiden können. Die Patienten sollen wissen, wie sie die frühen Symptome der Anaphylaxie erkennen, sodass sie rasch Hilfe anfordern und ihre Notfallmedikamente vorbereiten können. Obwohl keine randomisierten Studien vorliegen, gibt es Hinweise, dass ein individueller Aktionsplan für das Selbstmanagement die Gefahr eines Rückfalls verringert [153].

\section{Traumatisch bedingter Kreislaufstillstand}

\section{Allgemeines}

Ein Kreislaufstillstand infolge Trauma (TCA) hat eine sehr hohe Mortalität. Interessanterweise ist bei den überlebenden das neurologische Outcome viel besser als bei anderen Ursachen für einen Kreislaufstillstand [154, 155]. Die Reaktionszeit ist bei einem TCA entscheidend, und der Erfolg hängt von einer eingespielten überlebenskette $\mathrm{ab}$, die sich von einer erweiterten präklinischen Betreuung bis hin zur Behandlung in einem spezialisierten Traumazentrum spannt.

\section{Diagnose}

Die Diagnose eines TCA erfolgt klinisch: Der Traumapatient atmet nicht oder agonal, der Puls ist zentral nicht tastbar.

Ein Beinahekreislaufstillstand äußert sich durch eine instabile Kreislaufsituation, Hypotonie, Verlust der peripheren Pulse in den betroffenen Körperregionen und durch Verwirrung ohne offensichtliche Zentralnervensystemschädigung. Unbehandelt führt dieser Zustand wahrscheinlich zum Kreislaufstillstand. Eine standardisierte sonographische Sofortbeurteilung kann bei der initialen Diagnose und Therapie hilfreich sein, soll aber nicht die Durchführung der Wiederbelebungsmaßnahmen verzögern [156]. Kreislaufstillstände und Bewusstseinsverluste infolge internistischer oder neurologischer Erkrankungen (z. B. Hypoglykämie, Schlaganfall oder epileptische Anfälle) können sekundär zu einem Trauma führen. So ereignen sich beispielsweise gemäß verschiedenen Beobachtungsstudien ca. 2,5\% der außerklinischen Kreislaufstillstände in Fahrzeugen [157-159]. In diesen Fällen liegt häufig ein defibrillierbarer Rhythmus 
(VF/VT) vor [97]. Da diese medizinischen Kreislaufstillstände nach dem ALS-Universalalgorithmus behandelt werden müssen, ist es lebenswichtig, dass sie nicht fälschlicherweise als TCA interpretiert werden. Die medizinische Vorgeschichte, Hinweise auf Ereignisse unmittelbar vor dem Unfall und eine systematische Untersuchung (inklusive 12-Kanal-EKG) nach erfolgreicher Reanimation (ROSC) können helfen, die Ursache für den Kreislaufstillstand herauszufinden.

\section{Prognostische Faktoren und Ver- zicht auf Wiederbelebung}

Es gibt keine zuverlässigen Prädiktoren für die überlebenswahrscheinlichkeit nach TCA. Reagierende Pupillen, geordnete Rhythmen im EKG, respiratorische Aktivität, kurze CPR-Dauer und Prähospitalzeit werden mit einem positiven Outcome in Verbindung gebracht [159161]. Ein großer systematischer Review berichtete von einer überlebensrate von $3,3 \%$ nach stumpfem und 3,7\% nach penetrierendem Trauma, mit einem guten neurologischen Outcome in 1,6\% aller Fälle [154]. Bei Kindern ist die Prognose altersabhängig und günstiger als bei Erwachsenen [97, 154]. Die beträchtlichen Schwankungen der dokumentierten Mortalitätsraten (0-27\%) ergeben sich aus der Heterogenität der Fälle und der beteiligten Rettungseinrichtungen. PEA, primär als Ausdruck eines Low-outputZustands und Asystolie, sind die vorherrschenden Herzrhythmen bei TCA. Ein Kammerflimmern (VT) ist selten $\mathrm{zu}$ beobachten, weist aber die bessere Prognose auf [97, 155]. Eine Studie zeigte gutes neurologisches Outcome in 36,4\% der TCA mit VF als initialem Rhythmus, bei Vorliegen einer PEA hingegen nur in $7 \%$, bei einer Asystolie nur in 2,7\% der TCA [155]. Andere Untersuchungen fanden keine Überlebenden bei nicht defibrillierbaren Rhythmen [159, 162, 163].

Das „American College of Surgeons“ und die „National Association of EMS Physicians“ empfehlen den Verzicht auf Reanimationsmaßnahmen in Situationen mit feststellbarem oder unabwendbarem Todeseintritt und bei Traumapatienten, die apnoisch und pulslos ohne geordneten Herzrhythmus sind [164]. Und das, ob- wohl in solchen Fällen von Überlebenden mit gutem neurologischen Outcome berichtet wurde [155].

Wir empfehlen folgendes Vorgehen:

Ziehen Sie einen Verzicht auf eine Reanimation unter folgenden Bedingungen in Erwägung:

- keine Lebenszeichen in den vorangegangenen $15 \mathrm{~min}$,

- massives, mit dem Leben nicht vereinbares Trauma (z. B. Dekapitation, penetrierende Herzverletzung, sichtbarer Verlust von Hirngewebe).

Ziehen Sie eine Beendigung der Reanimationsmaßnahmen in Betracht, wenn:

- nach Behandlung der reversiblen Ursachen kein ROSC erreicht werden kann,

- sonographisch keine Herztätigkeit nachweisbar ist.

Da in Europa unterschiedliche Traumaversorgungssysteme existieren, empfehlen wir die Umsetzung von regionalen TCARichtlinien und die Anpassung der Behandlungspfade an die lokalen Infrastrukturen und Ressourcen.

\section{Behandlung}

Die Betonung der prompten Behandlung der potenziell reversiblen Pathologien bildet die Grundlage der Therapieleitlinien. Etliche Therapiealgorithmen berücksichtigen dieses Prinzip [97, 165167]. Alle Algorithmen sind darauf ausgerichtet, die reversiblen Gründe des TCA sowohl in der Präklinik wie auch in der Klinik früh zu erkennen und zu beheben. - Abb. 3 zeigt einen auf dem ALS-Universalalgorithmus basierenden Algorithmus zur Behandlung des traumatisch bedingten (Beinahe-)Herz-Kreislauf-Stillstands [168].

\section{Effektivität der Thoraxkompression}

Thoraxkompressionen sind noch immer die Standardmaßnahme bei Kreislaufstillständen sämtlicher Ätiologien. Ist dieser durch eine Hypovolämie, eine Perikardtamponade oder einen Spannungspneumothorax verursacht, so ist die Herzdruckmassage wahrscheinlich nicht so effektiv wie bei einem Kreislaufstillstand unter normovolämischen Verhält- nissen [169-172]). Aufgrund dieser Tatsache hat die Thoraxkompression eine geringere Priorität als die sofortige Behandlung der reversiblen Ursachen (z. B. Thorakotomie, Blutungskontrolle). In der Präklinik sollen vor Ort nur die erforderlichen lebensrettenden Interventionen durchgeführt und dann der Patient möglichst schnell in das nächste geeignete Krankenhaus transportiert werden.

\section{Hypovolämie}

In $48 \%$ aller TCA ist ein unkontrollierter Blutverlust Ursache für den Herz-Kreislauf-Stillstand [97]. Die Behandlung des schweren hypovolämischen Schocks weist verschiedene Elemente auf. Wichtigstes Prinzip dabei ist das verzögerungsfreie Erreichen einer Blutstillung, üblicherweise durch chirurgische oder radiologische Interventionen.

Eine vorübergehende Blutungskontrolle kann lebensrettend sein.

- Behandeln Sie komprimierbare Blutungen mit direktem Druck (mit oder ohne Verband), verwenden Sie Tourniquets, und/oder applizieren Sie hämostatische Substanzen topisch [173].

- Verwenden Sie bei schwieriger zu behandelnden, nicht komprimierbaren Blutungen Schienungen (z. B. Beckenschlinge), Blutprodukte, i.v.Volumengabe und Tranexamsäure (TXA), während Sie gleichzeitig eine chirurgische Blutstillung in die Wege leiten.

Im Verlauf der letzten 10 Jahre wurde das Konzept der "damage control resuscitation“ (DCR) Bestandteil der Behandlung der unkontrollierten, massiven Blutung. DCR kombiniert eine permissive Hypotonie und eine aggressive Gerinnungstherapie mit der "damage control surgery“, einer chirurgischen Schadenskontrolle, die darauf abzielt, die Verletzungen eines Patienten ohne zusätzliches Operationstrauma provisorisch zu stabilisieren. Bei begrenzter Evidenz besteht ein allgemeiner Konsens zu einer restriktiven i.v.-Flüssigkeitszufuhr, bis die Blutung chirurgisch gestillt ist [174]. Die permissive Hypotonie erlaubt es, nur gerade so viel Volumen zuzuführen, dass der Radialispuls erhalten bleibt $[175,176]$. 


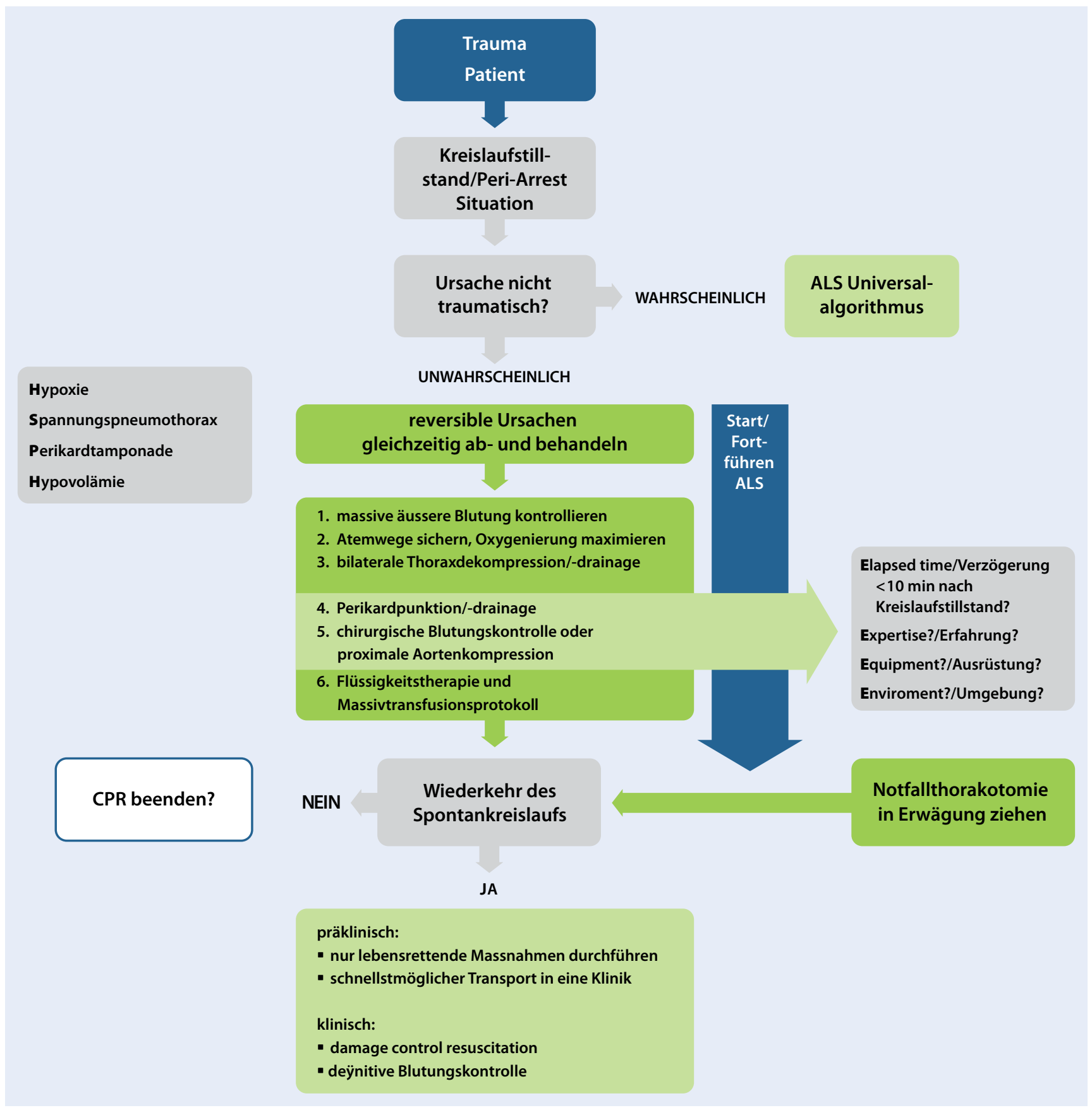

Abb. $3 \Delta$ Algorithmus für den Traumatisch bedingter Kreislaufstillstand

Unter aggressiver Gerinnungsstherapie versteht man den sehr frühen Einsatz von Blutprodukten, um das Verbluten durch eine traumatisch bedingte $\mathrm{Ge}$ rinnungsstörung zu verhindern [177]. Das empfohlene Verhältnis von Erythrozytenkonzentraten, FFP („fresh frozen plasma“) und Thrombozytenkonzentraten beträgt 1:1:1 [178]. Einige Rettungsdienste beginnen mit der Gabe von Blutprodukten bereits präklinisch $[179,180]$.
Gleichzeitige Anwendung einer „damage control surgery“ und einer aggressiven Gerinnungstherapie mit Verwendung von Massivtransfusionsprotokollen [173] sind Eckpfeiler der DCR bei Patienten mit lebensbedrohlich blutenden Verletzungen (• Abb. 4; [177]).

Obwohl die Evidenz bezüglich der permissiven Hypotonie (v. a. beim stumpfen Trauma) beschränkt ist, wird sie sowohl von militärischen wie zivilen Rettungsdiensten angewandt und ein systolischer Blutdruck von 80$90 \mathrm{mmHg}$ angestrebt [181]. Vorsicht ist angebracht bei Patienten mit einem Schädel-Hirn-Trauma, da diese aufgrund des erhöhten Hirndruckes einen höheren zerebralen Perfusionsdruck benötigen. Wegen der Gefahr von irreversiblen Organschädigungen soll die Dauer der permissiven Hypotonie 60 min nicht überschreiten [176].

TXA ( 1 g i.v. über 10 min als Loading Dose, gefolgt von einer Infusion von $1 \mathrm{~g}$ 


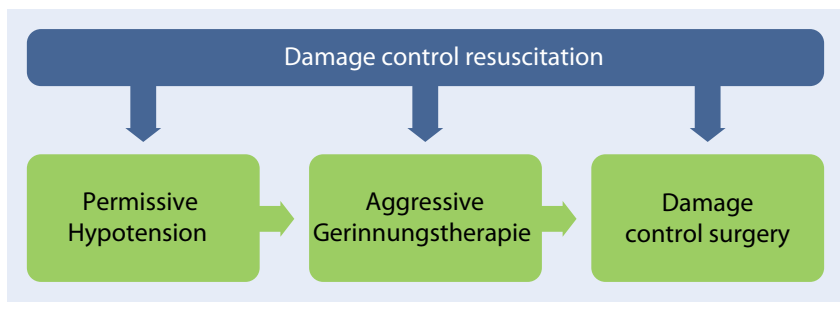

Abb. $4<$ Prinzipien der "damage control resuscitation" beim Trauma

über $8 \mathrm{~h}$ ) steigert die Überlebensrate bei traumatischen Blutungen [182]. Die Wirksamkeit ist in der ersten Stunde am größten, sicher aber innerhalb von $3 \mathrm{~h}$ nach dem Trauma. Verabreichen Sie TXA wenn möglich schon präklinisch.

\section{Hypoxie}

Hypoxämien durch Atemwegsverlegungen und stumpfe Thoraxkompressionstraumata sind offensichtlich für $13 \%$ aller TCA verantwortlich [97]. Ein effektives Atemwegsmanagement mit einer suffizienten Beatmung kann einen hypoxischen Kreislaufstillstand beheben. Die Gewährleistung und Aufrechterhaltung der Sauerstoffversorgung ist gerade bei Patienten mit schwer beeinträchtigten Atemwegen zwingend notwendig. Für Ungeübte weist die bei Verletzten oft schwierige Intubation eine hohe Misserfolgsquote auf [183, 184]. Gelingt die tracheale Intubation nicht auf Anhieb, verwenden Sie die grundlegenden Techniken des Atemwegsmanagements und supraglottische Atemwegshilfen der zweiten Generation, um die Oxygenation sicherzustellen.

Vor allem bei hypotonen Patienten kann eine Überdruckbeatmung durch Verminderung des venösen Rückstroms zum Herzen die Hypotonie verstärken [185]. Niedrige Atemhubvolumina und langsame Beatmungsfrequenzen helfen, den kardialen Preload zu optimieren. Überwachen Sie die Beatmung mittels Kapnographie, und streben Sie dabei eine Normokapnie an [173].

\section{Spannungspneumothorax}

Durch einen Spannungspneumothorax werden $13 \%$ aller TCA verursacht [97]. Führen Sie im Kreislaufstillstand zur Entlastung eine bilaterale Thorakostomie durch, die im Bedarfsfall zu einer bilateralen, queren Thorakotomie („Clamshell-Zugang“) erweitert werden kann. Bei überdruckbeatmeten Patienten sind einfache Thorakostomien wirksamer als Nadeldekompressionen und schneller durchgeführt als die Einlage einer Thoraxdrainage (vgl. Spannungspneumothorax, [186, 187]).

\section{Perikardtamponade und Notfall-} thorakotomie

Eine Perikardtamponade ist in ca. $10 \%$ der Fälle Ursache für einen TCA [97]. Liegt im Kreislaufstillstand ein penetrierendes Thorax- oder Oberbauchtrauma vor, kann eine Notfallthorakotomie ("Clamshell-Zugang“, [188]) das Leben des Patienten retten [189]. Kardiale Stichwunden weisen gegenüber Schussverletzungen eine 4 fach erhöhte Überlebenswahrscheinlichkeit auf [190]. Eine Notfallthorakotomie („resuscitative thoracotomy“, RT) kann auch bei anderen lebensbedrohlichen Verletzungen indiziert sein. Die diesbezügliche Evidenz wurde 2012 untersucht [191]. In den daraus resultierenden Leitlinien wird empfohlen, dass nach Krankenhauseinlieferung folgende Kriterien in die Entscheidung zur Notfallthorakotomie mit einbezogen werden sollen:

$$
\begin{aligned}
& \text { - stumpfes Trauma mit < } 10 \text { min prä- } \\
& \text { klinischer CPR, } \\
& \text { - penetrierendes Rumpftrauma mit } \\
& \text { <15 min CPR. }
\end{aligned}
$$

In den Leitlinien wird die Überlebensrate nach Notfallthorakotomie für alle Patienten mit ca. $15 \%$, für Patienten mit einer penetrierenden Herzverletzung mit ca. $35 \%$ beziffert. Demgegenüber wird bei Notfallthorakotomien nach stumpfem Trauma von miserablen überlebensquoten von 0-2\% berichtet [191, 192].

Der Erfolg der RT ist abhängig von ihrer zeitlichen Verzögerung. Eine englische Rettungseinrichtung empfiehlt die Durchführung einer RT vor Ort, falls bei Patienten mit penetrierenden Thoraxverletzungen nicht innerhalb von $10 \mathrm{~min}$ nach Eintreten der Pulslosigkeit eine chirurgische Intervention möglich ist [10]. Basierend auf diesem Ansatz überlebten von 71 vor Ort thorakotomierten Patienten 13, davon 11 mit gutem neurologischem Outcome.

Die Voraussetzungen für eine erfolgreiche RT können mit der 4E-Regel zusammengefasst werden:

- „Expertise“ (Erfahrung)

Teams, die eine RT durchführen, müssen von einer sehr gut ausgebildeten und kompetenten medizinischen Fachperson geführt werden und unter strukturierten Rahmenbedingungen arbeiten.

- „Equipment“ (Ausrüstung)

Eine adäquate Ausrüstung zur Durchführung der RT und Behandlung der intraoperativ erhobenen Befunde muss zwingend vorhanden sein.

- „Environment“ (Umgebung)

Idealerweise soll die RT in einem Operationssaal durchgeführt werden. Sie soll nicht stattfinden, wenn der Zugang zum Patienten erschwert oder das Zielkrankenhaus nicht leicht zu erreichen ist. - „Elapsed time“ (Zeitverzögerung)

Die Verzögerung vom Eintreten des Kreislaufstillstands bis zum Beginn der RT soll nicht mehr als 10 min betragen.

Ist eines dieser 4 Kriterien nicht erfüllt, so ist eine RT aussichtslos und gefährdet nur das Team [193].

Eine Perikardpunktion mit einer Nadel ist, egal ob mit oder ohne Ultraschallsteuerung, bei einer Perikardtamponade nicht Erfolg versprechend, weil das Blut im Perikardraum üblicherweise schon geronnen ist [194, 195]. Ist eine Thorakotomie aus irgendwelchen Gründen nicht möglich, so erwäge man eine ultraschallgesteuerte Perikardiozentese, um eine TCA mit vermuteter Perikardtamponade zu behandeln. Eine nicht durch bildgebende Verfahren gesteuerte Perikardiozentese ist nur dann eine Alternative, wenn kein Ultraschallgerät zur Verfügung steht. Einige Patienten können von der Platzierung einer Perikarddrainage profitieren. 


\section{Untersuchung(en)}

Kann die Ursache für einen Schock bei gefährdeten Traumapatienten nicht klinisch gefunden werden, soll eine Sonographie zur Steuerung der lebensrettenden Interventionen verwendet werden [173, 196]. Hämoperitoneum, Hämatooder Pneumothorax und die Perikardtamponade kann man so auch präklinisch innerhalb von wenigen Minuten diagnostizieren [197]. Eine frühe Ganzkörper-CT ist Teil der Erstbeurteilung („primary survey“) und verbessert das Outcome beim schweren Trauma [198]. Die Ganzkörper-CT wird vermehrt zur Erkennung der Schockursache und zur Steuerung der anschließenden Blutungskontrolle eingesetzt.

\section{Präklinische Versorgung}

Eine kurze Prähospitalisierungsphase führt zu einer höheren überlebensrate bei schwerem Trauma und TCA. Die Zeitverzögerung zwischen Verletzung und chirurgischer Blutungskontrolle soll auf ein Minimum reduziert und der Patient zur weiteren DCR möglichst sofort in ein Traumazentrum transferiert werden [173]. Für diese Patienten kann das Rettungskonzept „scoop and run“ lebensrettend sein.

\section{Spannungspneumothorax}

\section{Allgemeines/Einführung}

Ein Spannungspneumothorax, definiert als hämodynamische Beeinträchtigung/ Gefährdung durch eine sich ausdehnende intrapleurale Luftansammlung, ist eine der behandelbaren Ursachen eines Kreislaufstillstands und soll während der CPR ausgeschlossen werden [199]. Neben einem Trauma können Asthma und andere respiratorische Erkrankungen, aber auch medizinische Interventionen (z. B. Einlage eines zentralvenösen Katheters) zu einem Spannungspneumothorax führen. Er ist häufiger und dann oft schwerwiegender bei Patienten, die mit Überdruck beatmet werden [200]. Schwerverletzte Patienten, die im präklinischen Bereich behandelt werden, weisen eine Spannungspneumothoraxhäufigkeit von ca. $5 \%$ auf (in $13 \%$ dieser Fälle kommt es $\mathrm{zu}$ einem TCA). Bei erwachsenen Patienten, die auf einer Intensivstation betreut werden, beträgt diese Inzidenz $<1 \%[97,201,202]$.

\section{Diagnose}

Die Diagnose Spannungspneumothorax bei hämodynamisch instabilen Patienten oder im Kreislaufstillstand basiert auf der klinischen Untersuchung. Die Befunde umfassen Symptome der hämodynamischen Instabilität (Hypotonie, Kreislaufstillstand) und Hinweise auf einen Pneumothorax (vorausgegangene Atemnot, Hypoxie, einseitiges Fehlen von Atemgeräuschen bei der Auskultation, subkutanes Emphysem) oder eine Mediastinalverlagerung (Tracheadeviation und Stauung der Jugularvenen, [200]). Während der CPR sind diese Zeichen nicht immer klassisch. Beim Vorliegen einer schwerwiegenden Hypotonie oder eines Kreislaufstillstands führen Sie im Verdachtsfall noch vor einer radiologischen Bestätigung eine Entlastung durch [201].

\section{Behandlung}

\section{Nadeldekompression}

Die Nadeldekompression, die von beinahe allen Rettungsdienstmitarbeitern beherrscht wird, ist schnell durchführbar, aber von begrenztem Nutzen [203, 204].

Ein signifikanter Anteil der Patienten weist eine Brustwanddicke auf, die eine Nadeldekompression unter Verwendung einer 14-g-Kanüle mit Standardlänge ineffizient macht [205]. Die Kanülen neigen zum Knicken oder Verstopfen [206]. Auf jeden Versuch einer Nadeldekompression soll die Einlage einer Thoraxdrainage folgen (vgl. Asthma).

\section{Thorakostomie}

Eine effektive Behandlung des TCA bei Spannungspneumothorax umfasst tracheale Intubation, Überdruckbeatmung und Thoraxdruckentlastung. Die einfache Thorakostomie ist leicht durchzuführen und wird von verschiedenen Arzt besetzten Rettungsmitteln präklinisch routinemäßig angewandt [187, 207]. Die Inzision und das rasche Eröffnen des Pleuraraums bei überdruckbeatmeten Patienten entsprechen den ersten Schritten des Vorgehens bei Einlage der Standardthorax- drainage, die dann erst nach der primären Wiederbelebung platziert werden soll (vgl. TCA). Bei der Thoraxdrainage wird mehr Material und Zeit benötigt, und das Anlegen eines geschlossenen Systems birgt die Gefahr einer erneuten Luftretention. Thoraxdrainagen können verstopfen (Blutgerinnsel, Lungengewebe) oder abknicken.

\section{Perikardtamponade}

\section{Allgemeines}

Eine Perikardtamponade entsteht durch den Druck des flüssigkeitsgefüllten Herzbeutels, der die Herzfunktion einschränkt und schlussendlich zum Kreislaufstillstand führt. Am häufigsten geschieht dies nach penetrierenden Traumen oder herzchirurgischen Eingriffen. Die Mortalität ist hoch, und um eine Überlebenschance zu haben, ist eine sofortige Entlastung des Perikards notwendig.

\section{Behandlung}

\section{Thorakotomie}

Die Kriterien und Voraussetzung für eine Notfallthorakotomie bei Patienten mit einem penetrierenden Thorax- oder Oberbauchtrauma wurden im Kapitel über den traumatischen Kreislaufstillstand beschrieben.

\section{Perikardiozentese}

Ist die Durchführung einer Thorakotomie nicht möglich, erwägen Sie eine ultraschallgesteuerte Perikardiozentese, um eine TCA mit vermuteter traumatischer oder nicht traumatischer Perikardtamponade zu behandeln. Eine Perikardiozentese ohne Steuerung durch ein bildgebendes Verfahren ist nur dann eine Alternative, wenn kein Ultraschallgerät zur Verfügung steht.

\section{Thrombose}

\section{Lungenembolie}

\section{Allgemeines \\ Ein durch eine Lungenembolie ver- ursachter Kreislaufstillstand ist die schwerste Form der klinischen Manifestation einer venösen Thrombo- embolie, die ihren Ursprung in den}


meisten Fällen in einer tiefen Venenthrombose (TVT) hat [208]. Der Anteil der Kreislaufstillstände, die durch Lungenembolie bedingt sind, wird präklinisch mit 2-9\% [209-212] und innerklinisch mit 5-6\% [213, 214] angegeben, wird aber wahrscheinlich unterschätzt. Die Überlebensquote ist allgemein niedrig $[211,215]$. Spezifische Therapiemaßnahmen in diesen Situationen sind die Gabe von Fibrinolytika, die chirurgische Embolektomie und die perkutane mechanische Thrombektomie.

\section{Diagnose}

Während eines Kreislaufstillstands ist eine Lungenembolie schwierig $\mathrm{zu}$ diagnostizieren. Eine Studie berichtet über ein korrektes Erkennen der Ursachen in bis zu $85 \%$ der innerklinischen Wiederbelebungsversuche [214]. In der Präklinik stellt die Diagnose einer akuten Lungenembolie aber eine echte Herausforderung dar [212, 216].

Die Europäische Gesellschaft für Kardiologie hat 2014 in ihren Leitlinien zur Diagnose und zum Management der Lungenembolie eine „bestätigte Lungenembolie“ als „Diagnose-Wahrscheinlichkeit, die groß genug ist, die Notwendigkeit einer spezifischen Behandlung zu erkennen" definiert [208].

Anamnese, klinische Beurteilung, Kapnographie und Echokardiographie (falls verfügbar) können während der CPR alle mit unterschiedlicher Spezifität und Sensibilität zur Diagnose einer akuten Lungenembolie beitragen:

- Plötzliches Auftreten von Atemnot, pleuritische oder substernale Brustschmerzen, Husten, Hämoptyse, Synkope oder v. a. Zeichen einer TVT (einseitige Schwellung der unteren Extremitäten) sind häufige Symptome, die einem Kreislaufstillstand vorangehen [208]. Es kann aber auch sein, dass der Kreislaufstillstand als erstes Symptom der Lungenembolie auftritt [217].

- Holen Sie Auskünfte über die medizinische Vorgeschichte, prädisponierende Faktoren und Medikamente ein, obwohl es sich bei allen folgenden Hinweisen um unspezifische Zeichen für eine Lungenembolie handelt [208]:
- Lungenembolien oder TVT in der Anamnese,

- chirurgische Eingriffe oder Immobilisation in den letzten 4 Wochen,

- aktive Krebserkrankung,

- klinische Zeichen einer TVT,

- orale Antikonzeption oder Hormonsubstitution,

- Fernflüge.

Bei $30 \%$ der Patienten mit Lungenembolie gibt es keine offensichtlichen Risikofaktoren [218].

- Ist ein 12-Ableitungs-EKG vor Eintritt des Kreislaufstillstands vorhanden, können Zeichen der Rechtsherzbelastung gefunden werden:

- T-Negativierung in den Ableitungen V1-V4,

- QR-Muster in V1,

- S1Q3T3-Typ (markante S-Zacke in Ableitung I, Q-Zacke und negative T-Welle in Ableitung III),

- inkompletter oder kompletter Rechtsschenkelblock [208, 219].

- Der Kreislaufstillstand präsentiert sich üblicherweise als PEA [211].

- Niedrige $\mathrm{ETO}_{2}$-Werte (ca. $1,7 \mathrm{kPa} / 13 \mathrm{mmHg}$ ) unter laufender, qualitativ hochwertiger Thoraxkompression unterstützen die Diagnose Lungenembolie, sind aber kein spezifisches Zeichen [209].

- Ziehen Sie eine notfallmäßige Echokardiographie durch einen kompetenten Sonographeur als zusätzliches Diagnostik-Tool zur Erkennung einer Lungenembolie in Erwägung, falls diese ohne Unterbrechung der Thoraxkompression (z. B. während der Rhythmusanalyse) durchgeführt werden kann. Die echokardiographischen Befunde werden bei einem akuten Verschluss von $>30 \%$ des pulmonal arteriellen Gefäßbaums offensichtlich [220]. Häufiger echokardiographischer Befund ist ein vergrößerter rechter Ventrikel mit abgeflachtem interventrikulärem Septum [221, 222]. Ein Fehlen dieser Merkmale schließt aber die Diagnose einer Lungenembolie nicht aus [223]. Zeichen einer rechtsventrikulären Überlastung oder Dysfunktion können auch bei anderen kardialen oder pulmonalen Erkrankungen auftreten [224].

- Weitere, spezifischere Diagnostikmethoden, wie beispielsweise die DDimer-Bestimmung, die (computertomographische) pulmonale Angiographie, die Lungenszintigraphie oder die MRT, werden bei Vorliegen eines Kreislaufstillstands nicht empfohlen.

\section{Modifikationen der kardio- pulmonalen Wiederbelebung}

Eine Metaanalyse, die Patienten mit einer Lungenembolie als Ursache für den Kreislaufstillstand einschloss, folgerte, dass Fibrinolytika die ROSC-Rate, das Überleben bis zur Krankenhausentlassung und das neurologische Langzeitergebnis steigern [225]. Eine Subgruppenanalyse, die in einer randomisierten kontrollierten Studie mit Thrombolytika oder Placebo behandelte Patienten verglich, konnte bezüglich Überleben keinen Unterschied nachweisen [215]. Allerdings war diese Studie nicht für die Behandlung der Lungenembolie konzipiert und so angelegt, dass in dieser kleinen Subgruppe keine Signifikanz erreicht werden konnte.

Auch einige andere nicht randomisierte Studien haben sich mit der Verwendung von Thrombolytika bei der Behandlung des durch eine Lungenembolie verursachten Kreislaufstillstands befasst. Es konnte jedoch nur eine beschränkte Evidenz für eine verbesserte neurologische Erholung der Überlebenden gefunden werden $[211,226]$.

Halten Sie sich bei einem Kreislaufstillstand, der vermutlich durch eine Lungenembolie verursacht wurde, an die Standard-ALS-Leitlinien (vgl. „Erweiterte lebensrettende Maßnahmen für Erwachsene“, [168]).

Die Entscheidung zur Behandlung einer Lungenembolie muss früh, nämlich zu einem Zeitpunkt, wo ein gutes Outcome noch möglich ist, gefällt werden. Die folgenden Behandlungsmodifikationen werden empfohlen:

- Erwägen Sie die Verabreichung von

Fibrinolytika, falls eine Lungenembolie die bekannte oder vermutete Ursache für den Kreislaufstillstand ist. Eine fortdauernde CPR ist keine Kontraindikation zur Durchführung einer Fibrinolyse. Trotz erhöhtem 
Blutungsrisiko kann die Fibrinolyse eine effektive Behandlung sein, die ohne Zeitverzögerung sogar außerhalb der spezialisierten Gesundheitseinrichtungen gestartet werden kann. An Orten (z. B. in der Präklinik) ohne alternative Möglichkeiten überwiegt der potenzielle Nutzen der Fibrinolyse die möglichen Risiken im Hinblick auf eine verbesserte Überlebensrate [211, 227-231].

- Ist das fibrinolytische Medikament einmal verabreicht, soll die CPR für mindestens 60-90 min fortgeführt werden, bevor Sie die Wiederbelebungsversuche abbrechen [227, 232]. Es gibt Berichte von Überleben mit gutem neurologischem Outcome in Fällen, in denen die CPR > $100 \mathrm{~min}$ dauerte [233].

- Erwägen Sie die Verwendung von mechanischen Reanimationshilfen, wenn die Aufrechterhaltung einer qualitativ hochstehenden Thoraxkompression über einen längeren Zeitraum benötigt wird.

\section{Extrakorporale Reanimation}

Einige Beobachtungsstudien kommen zu dem Schluss, dass der Einsatz von extrakorporalen Kreislaufunterstützungssystemen (ECLS) angebracht ist, wenn der Kreislaufstillstand mit einer Lungenembolie einhergeht [234, 235]. Die Anwendung eines ECLS erfordert beträchtliche Ressourcen und Übung. Ziehen Sie den Einsatz in Erwägung, wenn die initialen erweiterten lebensrettenden Maßnahmen (ALS) keinen Erfolg gebracht haben und/oder um eine pulmonale Thrombektomie überhaupt zu ermöglichen.

\section{Chirurgische Embolektomie und mechanische Thrombektomie}

Das Überleben von Lungenemboliepatienten, bei denen während der Reanimation eine chirurgische Embolektomie durchgeführt wurde, wird in 2 Fallserien mit 13 und $71 \%$ angegeben. Diese Resultate wurden aber nicht mit der Standardtherapie verglichen $[229,236]$.

Im Kreislaufstillstand bei vermuteter Lungenembolie wird die Durchführung einer chirurgischen Embolektomie oder einer mechanischen Thrombektomie nicht empfohlen. Ist hingegen eine Lungenembolie nachweislich die Ursache des Kreislaufstillstands, soll die Anwendung dieser Methoden in Betracht gezogen werden.

\section{Perkutane pulmonale Thrombektomie}

In einer Fallserie war die perkutane pulmonale Thrombektomie während der Reanimation bei 6 von 7 Patienten erfolgreich $[237,238]$. Es sind aber größere Studien notwendig, um diese Methode $\mathrm{zu}$ validieren.

\section{Versorgung nach der Wieder- belebung}

Schließen Sie beim Patienten mit anhaltendem ROSC eine intraabdominale und intrathorakale reanimationsbedingte Verletzung aus, v. a. wenn mechanische Reanimationshilfen und gleichzeitig Fibrinolytika eingesetzt wurden [239241]. Versuchen Sie die Ursache für die Lungenembolie zu finden und zu behandeln. Evaluieren Sie die Risiken für das erneute Auftreten einer Lungenembolie, und leiten Sie die adäquaten Maßnahmen zur Verhinderung ein.

\section{Koronare Thrombose}

Die koronare Herzkrankheit ist die häufigste Ursache für eine Reanimation außerhalb des Krankenhauses. Das Management des ACS rund um die Wiederbelebung wird in einem separaten Kapitel abgehandelt (vgl. „Initiales Management des ACS“, [242]). In Kompetenzzentren für Herz-KreislaufStillstand können Verschlüsse und hochgradige Stenosen der Koronararterien identifiziert und behandelt werden. Von allen Patienten mit außerklinischem Kreislaufstillstand werden allerdings mindestens die Hälfte nicht ins Krankenhaus transportiert, wenn kein ROSC erreicht werden konnte (vgl. Kap. 11, „Ethik der Wiederbelebung und Entscheidungen am Lebensende", [243]). Obwohl beim bereits eingetretenen Kreislaufstillstand eine korrekte Diagnose der Ursache schwierig ist, liegt bei primärem Kammerflimmern wahrscheinlich eine koronare Herzkrankheit mit Verschluss eines großen Koronargefäßes vor.

Ziehen Sie den Transport unter laufender Reanimation in Erwägung, falls im Zielkrankenhaus Behandlungsmöglichkeiten zur Verfügung stehen, die präklinisch nicht durchgeführt werden können. Diese Therapieoptionen umfassen die sofortige Koronarangiographie, die primäre perkutane koronare Intervention („primary percutaneous coronary intervention “, PPCI) oder eher seltenere Eingriffe wie die pulmonale Embolektomie (vgl. „Lungenembolie“). Die Entscheidung für oder gegen einen solchen Transport ist komplex und abhängig von den lokalen Gegebenheiten. Der präklinische Einsatz von extrakorporalen Kreislaufunterstützungssystemen (ECLS) erfordert Spezialkompetenzen und konnte bisher nicht verbreitet eingeführt werden [244-246].

Mechanische Reanimationshilfen gewährleisten eine qualitativ hochwertige CPR während des Transports und der perkutanen koronaren Intervention (vgl. „Kreislaufstillstand in HEMS und Ambulanzflugzeug", [247, 248]).

Es gibt nur eine begrenzte Evidenz, den Transport ins Krankenhaus unter Reanimation routinemäßig zu empfehlen. Die Entscheidung ist abhängig von der Patientenauswahl, der Verfügbarkeit von optimalen Methoden zur mechanischen und hämodynamischen Unterstützung während des Transports in die Klinik und dort vom Management der zugrunde liegenden Pathologie, der Behandlung nach ROSC, der Komplikationsrate und dem Outcome. Es gibt keine großen Outcome Studien, allerdings deuten kleine Fallserien auf einen Nutzen in ausgewählten Fällen hin [249]. Bevor definitive Empfehlungen ausgesprochen werden können, müssen kontrollierte Studien durchgeführt werden [250].

Ein Transport unter CPR direkt ins Herzkatheterlabor kann infrage kommen, wenn die präklinische und klinische Infrastruktur verfügbar ist und die Teams Erfahrung mit den mechanischen und hämodynamischen Unterstützungssystemen und der notfallmäßigen PPCI unter Reanimation haben. Eine exzellente Kooperation zwischen den prä- und innerklinischen Teams ist erforderlich. Bei der Entscheidung zum Transport unter laufender Reanimation soll die realistische Überlebenschance [z. B. beobachteter Kreislaufstillstand mit initial 
defibrillierbarem Rhythmus (VF, pVT) und Ersthelfer-CPR] miteinbezogen werden. Ein intermittierender ROSC erleichtert die Entscheidung zum Transport sehr [251].

\section{Intoxikation}

\section{Allgemeine Überlegungen}

\section{Einleitung}

Insgesamt wird Kreislaufstillstand oder Tod nur selten durch Vergiftungen verursacht [252]. Dennoch ist eine Krankenhausaufnahme aufgrund von Vergiftung häufig, so gab es z. B. in Großbritannien 140.000 Aufnahmen in einem Jahr [252]. Vergiftungen durch therapeutische Substanzen, Genussdrogen oder Haushaltsmittel sind der häufigste Grund für eine Krankenhauseinlieferung oder eine Kontaktaufnahme zu einer Giftinformationszentrale. Unsachgemäße Medikamentendosierungen, Medikamentenwechselwirkungen oder Medikationsfehler können ebenso Schäden verursachen. Versehentliche Vergiftungen sind bei Kindern am häufigsten. Tötungsdelikte mit Medikamenten sind unüblich. Auch Industrieunfälle, Kriege oder Terrorismus können zu einer Exposition mit Giftstoffen führen. Die Evidenz für die Behandlungen beruht auf Tierversuchsstudien, Fallberichten und kleinen Fallserien [253-255].

\section{Verhinderung eines Kreislauf- stillstands}

Untersuchen Sie den Patienten systematisch nach dem ABCDE-Schema. Atemwegsverlegungen und nachfolgender Atemstillstand aufgrund einer Bewusstseinseintrübung sind häufige Todesursachen nach Eigenvergiftung (Benzodiazepine, Alkohol, Opioide, trizyklische Antidepressiva, Barbiturate, $[256,257])$. Eine frühzeitige endotracheale Intubation durch entsprechend geschulte Helfer kann das Risiko einer Aspiration vermindern. Intoxikationsbedingte Hypotension reagiert in der Regel auf i.v.-Flüssigkeitsgabe, gelegentlich sind Vasopressoren notwendig (z. B. Noradrenalin). Bestimmen Sie die Elektrolyte (v. a. Kalium), Blutzucker und Blutgase. Stellen Sie Blut- und Urinproben zur Analyse sicher. Patienten mit schweren Vergiftungen sollen auf einer Intensivstation versorgt werden [257].

\section{Modifikationen der kardio-} pulmonalen Wiederbelebung

- Legen Sie eine niedrige Schwelle für ihre persönliche Sicherheit an, wenn es sich um einen suspekten oder unerwarteten Kreislaufstillstand handelt, vor allem, wenn es sich um mehr als einen Betroffenen handelt.

- Vermeiden Sie Mund-zu-MundBeatmung bei Vergiftungen mit Chemikalien wie Cyaniden, Schwefelwasserstoffen, ätzenden Substanzen oder Organophosphaten.

- Behandeln Sie lebensbedrohliche Tachyarrhythmien mittels Kardioversion unter Beachtung der Periarrest-Algorithmen (s. Kap. 3 „ALS“). Die Therapie enthält die Korrektur von Elektrolyten und die Behandlung von Säure-Basen-Störungen (s. „Hypo-/Hyperkaliämie oder andere Elektrolytstörungen").

- Versuchen Sie, den Giftstoff zu identifizieren. Angehörige, Freunde und die Rettungsdienstbesatzung können hilfreiche Informationen liefern. Die Untersuchung der Patienten ergibt möglicherweise diagnostische Hinweise wie Gerüche, Nadeleinstichstellen, eine abnorme Pupillenreaktion oder Zeichen von Verletzungen oder Verätzungen im Mundraum.

- Messen Sie die Temperatur des Patienten, da Hypo- oder Hyperthermie nach einer Drogenüberdosierung auftreten kann (s. auch: „Hypo-/Hyperthermie“).

- Stellen Sie sich auf eine verlängerte Zeit für die Reanimationsmaßnahmen ein, insbesondere bei jungen Patienten, da der Giftstoff während der fortgesetzten Reanimation metabolisiert oder ausgeschieden werden könnte.

- Alternativ kann bei schweren Intoxikationen nützen: eine höhere Dosis von Medikamenten als üblich (z. B. hochdosierte Insulingabe), unübliche Therapien (z. B. i.v.-Lipidinfusion, [259-262]), eine verlängerte
Reanimationsdauer, extrakorporale Kreislaufunterstützung (ECLS, [263, 264]) und Hämodialyse.

- Kontaktieren Sie regionale oder nationale Giftinformationszentralen für weitere Therapieempfehlungen. Eine internationale Übersicht findet sich im Internet unter: http://www. who.int/ipcs/poisons/centre/en/ [2].

- Folgende Onlinedatenbank mit Informationen über Gift- und Gefahrenstoffe kann hilfreich sein: http://toxnet.nlm.nih.gov/.

\section{Spezifische therapeutische Maßnahmen}

Es gibt einige spezifische therapeutische Methoden für Vergiftete, die direkt hilfreich sind und das Outcome verbessern. Dies sind: Dekontamination, gesteigerte Ausscheidung/Elimination und der Einsatz spezieller Antidote [265-267]. Viele dieser Interventionen sollen nur nach Rücksprache mit Experten erfolgen. Für aktuelle und direkte Empfehlungen bei schweren oder unbekannten Vergiftungen sind die Vergiftungszentralen zu kontaktieren.

\section{Dekontamination}

Dekontamination ist der Prozess, bei dem der Giftstoff vom oder aus dem Körper des Betroffenen, abhängig vom Aufnahmeweg, entfernt wird:

- Bei Aufnahme des Giftstoffs über die Haut besteht das initiale Management im Enfernen der Kleidung und dem ausgiebigen Spülen mit Wasser. Dies gilt nicht, wenn es sich bei dem Giftstoff um reaktive Alkalimetalle handelt.

- Die routinemäßige Magenspülung zur gastralen Dekontamination wird nicht länger empfohlen. In seltenen Fällen (z. B. tödliche Vergiftung mit kurz zurückliegender Aufnahme) soll die Anwendung nur durch ausgebildete und erfahrene Personen durchgeführt werden. Eine Magenspülung kann mit lebensbedrohlichen Komplikationen verbunden sein. Dies sind Lungenentzündung, Ösophagusoder Magenperforation, Flüssigkeitsund Elektrolytverschiebungen und Rhythmusstörungen. Die Magenspülung ist kontraindiziert, wenn 
keine Sicherung des Atemwegs erfolgt ist oder wenn ein Kohlenwasserstoff mit hoher Aspirationsgefahr oder eine ätzende Substanz eingenommen wurde [267, 268].

- Die bevorzugte Methode zur gastralen Dekontamination bei sicherem oder gesichertem Atemweg ist die Aktivkohlegabe. Diese ist am effektivsten, wenn die Gabe innerhalb von $1 \mathrm{~h}$ nach Ingestition erfolgt [269]. Aktivkohle bindet kein Lithium, Schwermetalle oder toxischen Alkohol. Die üblichen Nebenwirkungen sind Erbrechen und Obstipation. Es liegen nur eingeschränkte Informationen über eine Verbesserung des Outcomes durch Aktivkohle vor [257].

- Untersuchungen an Freiwilligen haben ergeben, dass bei Vergiftungen mit Medikamenten mit verzögerter Freisetzung, oder bei magensaftresistent beschichteten Medikamenten eine Magen-Darm-Spülung in Betracht gezogen werden kann, speziell bei einem Therapiebeginn später als 2 h nach Einnahme, wenn Aktivkohle nur noch bedingt effektiv ist,. Diese kann auch sinnvoll sein zur Entfernung größerer Mengen von Eisen, Lithium, Kalium oder Drogenpaketen („bodypacking“). Die Magen-Darm-Spülung ist kontraindiziert bei Patienten mit MagenDarm-Obstruktionen, Perforationen, Darmverschluss oder hämodynamischer Instabilität [270].

- Vermeiden Sie den routinemäßigen Einsatz von Laxantien oder Emetika (z. B. nie Ipecac-Sirup, [271-273]).

\section{Gesteigerte Elimination}

Die Methoden, mit denen ein Giftstoff aus dem Körper entfernt werden kann, nachdem er absorbiert wurde, sind die mehrfache Gabe von Aktivkohle („multipledose activated charcoal“, MDAC), die Alkalisierung des Urins und extrakorporale Elimination.

- Mehrfache Gabe von Aktivkohle (MDAC) über mehrere Stunden kann die Drogenelimination steigern [274, 275]. Es existiert keine Standarddosierungsempfehlung für MDAC
(50-100 g für den Erwachsenen, 10$25 \mathrm{~g}$ für Kinder $<5$ Jahre).

- Eine Urinalkalisierung (Urin-pH $>7,5$ ) erfolgt über eine i.v.-Natriumbicarbonat-Infusion. Diese wird üblicherweise bei Patienten mit Salicylat-Intoxikationen eingesetzt, bei denen keine Indikation für eine Dialyse besteht. Denken Sie an Urinalkalisierung mit hoher Urinausscheidung (um die $600 \mathrm{ml} / \mathrm{h}$ ) bei schweren Vergiftungen mit Phenobarbital oder Insektenschutzmitteln (2,4-Dichlorphenoxyessigsäure oder Methylchlorphenoxypropionsäure, MCPP). Eine Hypokaliämie ist eine häufige Komplikation [265].

- Die Hämodialyse entfernt Giftstoffe oder Metaboliten mit niedrigem Molekulargewicht, niedriger Proteinbindung, niedrigem Verteilungsvolumen und hoher Wasserlöslichkeit. Bei Hypotension kommen kontinuierliche venovenöse Hämofiltration (CVVH) oder kontinuierliche venovenöse Dialyse (CVVHD) als Alternative zur Anwendung [257].

\section{Spezielle Giftstoffe}

Diese Guidelines betrachten nur die üblichen Giftstoffe, die einen Kreislaufstillstand auslösen können.

\section{Benzodiazepine}

Eine Überdosierung mit Benzodiazepinen kann zu Bewusstlosigkeit, Atemdepression und Hypotension führen. Flumazenil, ein kompetitiver Antagonist am Benzodiazepin-Rezeptor, kann zur Aufhebung der Sedatierung durch Benzodiazepine eingesetzt werden, solange kein Risiko für Krampfanfälle besteht. Die Antagonisierung einer Benzodiazepin-Vergiftung mit Flumazenil kann bei Patienten mit Benzodiazepin-Abhängigkeit oder der gleichzeitigen Einnahme von prokonvulsiven Medikamenten wie z. B. trizyklische Antidepressiva zu signifikanten Nebenwirkungen führen (Krampfanfälle, Arrhythmien, Hypotension und Entzugssymptomatik [276-278]). Der routinemäßige Einsatz von Flumazenil bei komatösen Patienten mit einer Überdosierung wird nicht empfohlen.

Es sind keine spezifischen Modifikationen des ALS-Algorithmus bei durch Benzodiazepin verursachten Kreislaufstillständen nötig [278-282].

\section{Opioide}

Eine Opiatvergiftung verursacht eine Atemdepression, gefolgt von einer Ateminsuffizienz oder einem Atemstillstand. Die Wirkung von Opiaten auf die Atmung ist schnell durch Naloxon antagonisierbar.

Bei schwerwiegenden Atemdepressionen durch Opioide, kommt es zu weniger unerwünschten Ereignissen wenn, bevor Naloxon gegeben wird, die Atemwege freigemacht werden, Sauerstoff gegeben und beatmet wird [283-289]. Die Naloxon-Anwendung kann die Notwendigkeit einer Intubation vermeiden. Die bevorzugte Applikation von Naloxon richtet sich nach den Erfahrungen und Qualifikationen des Rettungsdienstpersonals: Es sind sowohl i.v. als auch i.m., subkutane (s.c.), i.o. und nasale/ bukale Applikationen möglich [290, 291]. Die Applikationswege, die keinen i.v.Zugang nutzen, können schneller sein, da keine Zeit für einen solchen Zugang, der bei Drogenabhängigen schwierig sein kann, benötigt wird. Die initiale Dosis von Naloxon beträgt 0,4-2 mg bei i.v.-, i.o.-, i.m.- oder s.c.-Anwendung und kann alle 2-3 min wiederholt werden. Zusätzliche Dosen könnten alle 20-60 min notwendig sein. Die intranasale Dosis beträgt $2 \mathrm{mg}$ (jeweils $1 \mathrm{mg}$ pro Nasenloch) und kann alle 5 min wiederholt werden. Titrieren Sie die Dosis, bis der Patient adäquat atmet und sichere Atemwegsreflexe zeigt. Hohe Opioidüberdosierungen benötigen bis zu einer Gesamtdosis von 10 mg Naloxon [283-285, 290-300]. Alle Patienten, die Naloxon erhalten haben, müssen weiter überwacht werden.

Ein akuter Opioidentzug führt zu einem Zustand mit sympatomimetischer Überreaktion und kann Komplikationen wie Lungenödem, ventrikuläre Arrhythmien und schwere Agitation hervorrufen. Die Anwendung von Naloxon zur Aufhebung einer Opioidintoxikation soll bei Patienten mit Verdacht auf Opioidabhängigkeit vorsichtig erfolgen.

Es existieren keine Daten für zusätzlich zur ALS-Versorgung notwendige Therapien bei opioidbedingtem Kreislaufstillstand. Bei Atemstillstand existiert 
eine gute Evidenz für die Anwendung von Naloxon, aber nicht für irgendeine andere Ergänzung oder Änderung bei weiteren Interventionen [284].

\section{Trizyklische Antidepressiva}

In diesem Abschnitt werden sowohl trizyklische als auch andere zyklische Drogen (Amitriptyline, Desipramine, Imipramine, Nortriptyline, Doxepin und Clomipramine) betrachtet. Die Selbstvergiftung durch trizyklische Antidepressiva ist häufig und kann zu Hypotension, Krämpfen, Koma und lebensbedrohlichen Rhythmusstörungen führen. Kardiotoxische Wirkung, bedingt durch anticholinerge und $\mathrm{Na}$-Kanal blockierende Effekte kann eine Breitkomplextachykardie (VT) verursachen. Eine Hypotension wird verstärkt durch eine $\alpha_{1}$-Rezeptorblockade. Anticholinerge Effekte sind Mydriasis, Fieber, trockene Haut, Delir, Tachykardie, Darmverschluss (Ileus) und verminderte Urinausscheidung. Die meisten lebensbedrohlichen Situationen treten in den ersten $6 \mathrm{~h}$ nach Giftaufnahme auf [301-303].

Eine QRS-Komplexverbreiterung (>100 ms) und eine Lageveränderung im Sinne eines Rechtslagetyps weisen auf ein höheres Risiko für Arrhythmien hin [304306]. Bei durch trizyklische Substanzen verursachte ventrikuläre Arrhythmien kommt Natriumbicarbonat (1-2 mmol/ kgKG) zur Anwendung [307-312]. Obwohl keine Studien einen optimalen arteriellen Ziel-pH untersucht haben, wird ein $\mathrm{pH}$-Wert zwischen 7,45 und 7,55 als Zielwert empfohlen [255, 257]. Die Gabe von Natriumbicarbonat kann Arrhythmien und eine Hypotension verursachen, die möglicherweise unabhängig von einer Azidose auftreten [312].

I.v.-Lipidinfusionen haben im Rahmen von experimentellen Versuchen bei Trizyklikaintoxikationen geholfen, aber es liegen nur wenige Daten für Menschen vor [313,314]. Antitrizyklische Antikörper waren in experimentellen Modellen zur Kardiotoxizität von trizyklischen Substanzen ebenfalls vorteilhaft [315320]. Eine kleine Studie am Menschen beschrieb die Sicherheit der Anwendung, konnte jedoch keinen klinischen Vorteil zeigen [321].
Es liegen keine randomisierten kontrollierten Studien zur Behandlung eines Kreislaufstillstands durch trizyklische Giftstoffe vor. Eine kleine Fallserie zeigte einen Vorteil bei der Anwendung von Natriumbicarbonat, aber der Begleitumstand, dass Physiostigmin ebenfalls gegeben wurde, verhindert die Generalisierung der Ergebnisse [322].

\section{Kokain}

Eine sympathomimetische Überstimulation im Zusammenhang mit einer Kokainvergiftung kann zu Agitation, Tachykardie, hypertensiven Krisen, Hyperthermie und koronarer Vasokonstriktion mit nachfolgender myokaridaler Ischämie und Angina pectoris führen.

Bei Patienten mit schwerer kardiovaskulär wirkender Vergiftung können Alpha-Blocker (Phenotalamin, [323]), Benzodiazepine (Lorazepam, Diazepam, [324, 325]), Calcium-Kanal-Blocker (Verapamil, [326]), Morphin [327] und sublinguales Nitroglyzerin [328, 329] notwendig sein, um Hypertension, Tachykardie, myokardiale Ischämie und Agitation unter Kontrolle zu bringen. Die Evidenz für oder gegen den Einsatz von Beta-Blockern [330-333] inklusive solcher mit alpha-blockierender Potenz (Carvedilol und Labetolol, [334336]) ist begrenzt. Die optimale Auswahl eines Antiarrhythmikums für die Behandlung einer kokaininduzierten Tachyarrhythmie ist nicht bekannt. Bei Kreislaufstillstand wird nach den Standardwiederbelebungsleitlinien vorgegangen [337].

\section{Lokalanästhetika}

Die systemische toxische Wirkung von Lokalanästhetika bezieht sich auf das kardiovaskuläre und das zentrale Nervensystem. Schwere Agitation, Bewusstlosigkeit, Krämpfe, Bradykardie, Asystolie oder ventrikuläre Tachykardien können auftreten. Die Vergiftung zeigt sich typischerweise im Zusammenhang mit einer Regionalanästhesie, wenn ein Bolus des Lokalanästhetikums versehentlich i.v. oder i.a. verabreicht wird (s. auch "perioperativer Kreislaufstillstand").

Obwohl es mehrere Fallberichte und Fallserien von Patienten über die erfolg- reiche Reanimation nach einer intravenösen Gabe von Lipidlösungen gibt, bleibt die Evidenz für einen Vorteil der Behandlung von lokalanästhetikainduzierten Kreislaufstillständen limitiert. Auch wenn keine Daten vorliegen, können Patienten mit einem Kreislaufkollaps oder -stillstand von einer i.v. $20 \%$-Lipidlösung als Ergänzung zur Standardreanimation profitieren [338-352]. Geben Sie einen initialen Bolus von $20 \%$ Lipidlösung in der Dosierung von 1,5 ml/ $\mathrm{kgKG}$ über eine Minute und nachfolgend $15 \mathrm{ml} / \mathrm{kgKG} / \mathrm{h}$. Geben Sie maximal zwei weitere Boluswiederholungen in 5-minIntervallen und fahren Sie fort, bis der Patient sich entweder stabilisiert oder eine maximale kumulative Dosis von $12 \mathrm{ml} /$ kgKG erreicht wurde [259-262, 353].

Die Standardmedikation bei Kreislaufstillstand (z. B. Adrenalin) soll entsprechen der ALS-Leitlinien verabreicht werden, obwohl Tierversuche inkonsistente Evidenz für deren Wirkung bei Lokalanästhetikaintoxikationen ergeben haben [349, 352, 354-356].

\section{Beta-Blocker}

Die Beta-Blockertoxizität-bedingten Bradyarrhythmien und negativen inotropen Effekte sind schwierig zu behandeln und können zu einem Kreislaufstillstand führen.

Die Evidenz für die Behandlung basiert auf Fallberichten und Tierversuchen. Therapieerfolge sind beim Einsatz von Glucagon (50-150 $\mu \mathrm{g} / \mathrm{kgKG}$, [357-370]), hochdosierter Insulin- und Glukosegabe [371-373], Lipidemulsionen [374377], Phosphodiesterasehemmern [378, 379], extrakorporaler Unterstützung und IAPB-Einsatz (intraaortale Ballonpumpe, [380-382]) sowie Calciumsalzen [258, 383] beschrieben worden.

\section{Calciumkanalblocker}

Überdosierungen von Calciumkanalblockern nehmen als Ursache für einen Tod durch verschreibungspflichtige Medikamente zu [384, 385]. Eine Überdosierung von kurz wirksamen Medikamenten kann zügig in einen Kreislaufstillstand übergehen. Die mit Retardpräparaten führt möglicherweise zu einem verzögerten Auftreten von Arrhythmien, Schock und plötzlichem Kreislauf- 
zusammenbruch. Die Behandlung von Calciumkanalblockerintoxikationen wird nur unzureichend durch eine niedrige Evidenzqualität untermauert [386].

Geben Sie Calciumchlorid 10\% als 20-ml-Bolus (oder eine äquivalente Dosis von Calciumgluconat) alle 2-5 min bei schwerwiegender Bradykardie oder Hypotension und schließen Sie, soweit notwendig, eine Infusionstherapie an [255, 257, 258, 386, 387]. Während hohe Dosierungen von Calcium einige Nebenwirkungen beseitigen können, stellen sie einen normalen Kreislaufs selten wieder her. Eine hämodynamische Instabilität kann auf eine Hochdosistherapie mit Insulin ( $1 \mathrm{IE} / \mathrm{kgKG}$ und nachfolgend 0,5-2 IE/ $\mathrm{kgKG} / \mathrm{h})$ in Kombination mit Glukosesubstitution, Elektrolytmonitoring und dem Standardvorgehen mittels Flüssigkeit und Vasopressoren (z. B. Dopamin, Noradrenalin, Vasopressin) ansprechen [386-398].

Der Einsatz eines extrakorporalen Unterstützungssystems (ECLS) war verbunden mit einer gesteigerten Überlebensquote bei Patienten im schweren Schock oder mit Kreislaufstillstand, wobei Extremitätenischämien, Thrombosen und Blutungen in Kauf genommen wurden [264]. Studien zur Dekontamination, 4-Aminopyridinen, Atropin, Glucagon, Schrittmachertherapie, Levosimendan oder Plasmaaustausch zeigten unterschiedliche Ergebnisse [386].

\section{Digoxin}

Obwohl Intoxikationen mit Digoxin seltener vorkommen als eine Überdosierung von Calciumkanal- oder BetaBlockern, ist die Mortalität bei DigoxinÜberdosierung deutlich höher. Andere Medikamente wie beispielsweise Calciumkanalblocker und Amiodaron haben auch häufig einen Anstieg der Plasmakonzentration von Digoxin zur Folge. Atrioventrikuläre Überleitungsstörungen und ventrikuläre Übererregbarkeit, basierend auf Digoxin-Vergiftungen, können zu schwerwiegenden Rhythmusstörungen und Kreislaufstillstand führen.

Eine spezifische Antidottherapie mittels Digoxin-spezifischen Antikörperfragmenten (Digoxin-Fab) soll bei Arrhythmien mit hämodynamischer Instabilität eingesetzt werden [257, 399-
401]. Die Therapie mit Digoxin-Fab kann ebenso bei Vergiftungen durch Pflanzen (z. B. Oleander) oder chinesische Heilkräuter, die Herzglykoside enthalten, effektiv sein [399, 402, 403]. DigoxinFab interferiert mit der DigoxinSpiegelbestimmung durch Immunoassay und kann zur Überschätzung des PlasmaDigoxin-Spiegels führen. Bei akuter Vergiftung geben Sie einen initialen Bolus von 2-10 (unbekannte Digoxin-Dosis) Durchstichfläschchen Digoxin-Fab (38 mg pro Durchstechflasche) und wiederholen dies je nach Notwendigkeit [401]. Bei Kreislaufstillstand kann eine Gabe von 2-10 Durchstichfläschchen innerhalb von 30 min erwogen werden.

\section{Zyanide}

Zyanide sind im Allgemeinen ein seltener Grund für eine akute Vergiftung. Nichtsdestotrotz kommt eine Zyanidexposition relativ häufig bei Patienten mit Rauchgasinhalation im Zusammenhang mit Wohnungs- oder Industriebränden vor. Zyanide werden ebenso für einige chemische oder industrielle Prozesse angewendet. Die eigentliche Toxizität resultiert aus einer Inaktivierung der Zytochromoxidase (am Zytochrom A3). Hierdurch entkoppelt es die mitochondriale oxidative Phosphorylierung und behindert die Zellatmung auch bei ausreichendem Sauerstoffangebot. Gewebe mit dem höchsten Sauerstoffbedarf (Herz und Gehirn) sind durch eine akute Zyanidintoxikation am schwersten betroffen.

Patienten mit schwerer kardiovaskulärer Vergiftung (Kreislaufstillstand, Kreislaufinstabilität, metabolische Azidose oder eingeschränkter Bewusstseinsstatus) und gesicherter oder vermuteter Zyanidvergiftung sollen eine Zyanid-Antidottherapie zusätzlich zur Standardreanimation mit Sauerstoff erhalten. Die initiale Therapie soll einen Zyanidradikalenfänger enthalten (z. B. Hydroxocobalamin $100 \mathrm{mg} / \mathrm{kgKG}$ i.v. oder Nitrit (Natriumnitrit i.v. oder inhalatives Amylnitrit), und nachfolgend muss so schnell wie möglich Natriumthiosulfat i.v. verabreicht werden [404410]. Hydroxocobalamin und Nitrite sind gleich effektiv, Hydroxocobalamin scheintsicherer zu sein, da keine
Methämoglobinbildung und hypotensive Nebenwirkungen beschrieben sind [411413].

Beim Kreislaufstillstand durch Zyanid wird das Standardvorgehen keine Spontanzirkulation wiederherstellen, solange die Zellatmung blockiert ist. Zur Reaktivierung der Zytochromoxidase ist eine Antidotgabe notwendig.

\section{Kohlenmonoxid}

Kohlenmonoxidvergiftungen sind häufig. In den USA sind über 25.000 Krankenhausaufnahmen, bedingt durch Kohlenmonoxidvergiftung, pro Jahr beschrieben worden [414]. Die Kohlenmonoxidspiegel korrelieren nicht mit dem Auftreten initialer Symptome oder dem späteren Outcome. Patienten mit kohlenmonoxidbedingtem Kreislaufstillstand überleben selten, auch wenn vorher ein ROSC erreicht werden konnte [413, 416].

Die hyperbare Oxygenierung ist zur Behandlung von Kohlenmonoxidexposition und zur Reduktion von negativem neurologischem Outcome eingesetzt worden [417]. Dennoch versagten zwei CochraneAnalysen beim Versuch, einen positiven Effekt der hyperbaren Oxygenierung bei Kohlenmonoxidvergiftung zu bestätigen [416, 418]. Die Rolle von Kohlenmonoxid in der Stickoxidfreisetzung, in der reaktiven Sauerstoffformation und der direkten Wirkung auf die Ionenkanäle scheint signifikanter zu sein als die höhere Affinität zu Hämoglobin, das mit Sauerstoff therapiert wird [419]. Da der Vorteil des Transports eines kritisch kranken Patienten nach erfolgreicher Reanimation zu einem Hyperbaren Therapiezentrum nicht erwiesen ist, muss hier jeweils von Fall zu Fall entschieden werden [413, $416,418,419]$ in jedem Fall muss der Patient aber frühzeitig hohe Sauerstoffkonzentrationen erhalten. Schwangerschaft und kardiale Ischämie bleiben strenge Indikationen für die hyperbare Therapie. Patienten mit myokardialer Schädigung durch Kohlenmonoxid haben ein erhöhtes Risiko für kardiale und Gesamtmortalität, und zwar bis zu 7 Jahre nach der Vergiftung, weshalb eine kardiologische Folgeuntersuchung für diese Patienten sinnvoll ist [413, 420, 421]. 


\section{B. Besonderes Umfeld}

\section{Kreislaufstillstand in medizinischen Einrichtungen}

\section{Perioperativer Kreislaufstillstand}

\section{Einleitung}

Obwohl die Sicherheit bei chirurgischen Routineeingriffen über die letzten Jahrzehnte angestiegen ist, führen die höhere Anzahl von durchgeführten Eingriffen, insbesondere bei häufiger älteren Patienten und in Notfallsituationen, zu einer mehr oder weniger stabilen Inzidenz von perioperativen Kreislaufstillständen in der vergangenen Dekade.

Die Prinzipien der Therapie bleiben gleich, obwohl sich die Bedingungen bei einem perioperativen Kreislaufstillstand meist von denen sonstiger Kreislaufstillstände bei Krankenhauspatienten unterscheiden. Ein perioperativer Kreislaufstillstand kann durch die zugrunde liegende Ursache, physiologische Effekte des chirurgischen Eingriffs, Anästhetika und Infusionen, Komplikationen, basierend auf Komorbiditäten oder unerwünschte Ereignisse begründet sein.

\section{Epidemiologie}

Die übergreifende Inzidenz von perioperativen Kreislaufstillständen schwankt zwischen 4,3 und 34,6/10.000 Eingriffen [422-424]. Die große Breite beschreibt die Unterschiede im Case-Mix (es werden u. a. Neonaten und/oder herzchirurgische Patienten einbezogen) und in der Definition des Begriffs „perioperativ“. Die Inzidenz liegt bei Hochrisikopatienten, wie z. B. älteren Menschen, höher und wurde mit 54,4/10.000 Fälle beschrieben [425]. Bei Patienten, die sich einem Notfalleingriff unterziehen, liegt die Inzidenz bei 163/10.000 Fälle [426]. Junges Alter $<2$ Jahren, kardiovaskuläre oder respiratorische Komorbiditäten, höherer ASA-Status, perioperativer Schock und der chirurgische Eingriff als solches sind als Risikofaktoren für einen perioperativen Kreislaufstillstand identifiziert worden [426].

Die Inzidenz von Kreislaufstillständen, die primär anästhesiebedingt sind, stellt einen relativ kleinen Anteil in der Gesamtinzidenz dar, sie wird in Studien mit 1,1 bis
$3,26 / 10.000$ Eingriffen angegeben [425, $427,428]$. Das Gesamtüberleben bei perioperativem Kreislaufstillstand ist höher als bei präklinischen Kreislaufstillständen und wird mit Entlassungsraten von 3036,6 beschrieben [422, 424, 428].

Vollnarkose versus Regionalanästhesie Die Inzidenz eines perioperativen Kreislaufstillstands während einer Vollnarkose ist höher als bei Regionalanästhesieverfahren. Nach einer Studie der MayoKlinik war sie bei Patienten, die eine Vollnarkose erhielten, bis zu 3-mal (4,3/10.000 Eingriffe) höher als bei jenen, die lokal anästhesiert worden waren. Im Allgemeinen ist die Inzidenz jedoch über einen Zehnjahreszeitraum signifikant gesunken [423]

\section{Ursachen des Kreislaufstillstands}

Als allgemeine Ursachen für einen Kreislaufstillstand konnten identifiziert werden:

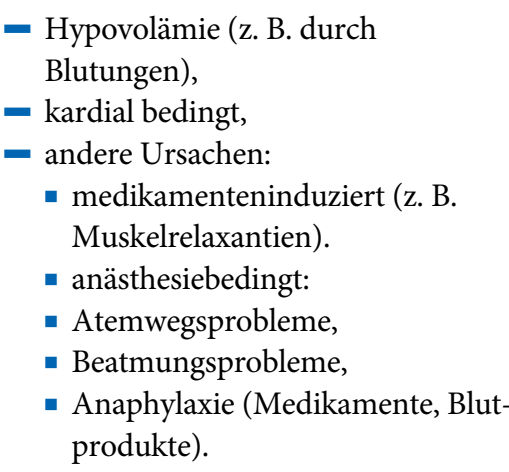

Der häufigste Grund für einen anästhesiebedingten Kreislaufstillstand liegt im Atemwegsmanagement [427, 428]. Unmögliche Beatmung, medikamentenbedingte Ereignisse, Komplikationen bei der Anlage eines zentralen Venenkatheters oder ein perioperativer Myokardinfarkt spielen ebenfalls eine Rolle [423, 429]. Bei Kindern kommen Atemwegsverlegung durch Laryngospasmus, Hypovolämie durch Blutverlust und Hyperkaliämie nach Transfusion länger gelagerter Blutkonserven als Ursachen hinzu [430].

Ein Kreislaufstillstand aufgrund von Blutverlust hatte bei den nicht herzchirurgischen Eingriffen die höchste Mortalität, nur 10,3\% dieser Patienten verlassen das Krankenhaus lebend [423]. In einer Untersuchung der Mayo-Klinik war der Anfangsrhythmus beim perioperativen Kreislaufstillstand Asystolie in $41,7 \%$, Kammerflimmern in $35,4 \%$, PEA in $1,4 \%$, und in $8,5 \%$ der Fälle war er unbekannt. Im Gegensatz zu Untersuchungen über Kreislaufstillstand i. Allg. war der Rhythmus mit der besten Überlebenschance die Asystolie (43\% Überleben, $[423,431])$.

\section{Vorgehen bei perioperativem Kreislaufstillstand}

Normalerweise sind Patienten im Operationssaal komplett monitorüberwacht, und es sollte nur eine geringe oder gar keine Verzögerung bei der Diagnose eines Kreislaufstillstands entstehen. Bei Hochrisikopatienten wird der Blutdruck oft invasiv kontrolliert, unbezahlbar bei einem Kreislaufstillstand. Ist ein Kreislaufstillstand sehr wahrscheinlich, sollen vor der Narkoseeinleitung Defibrillationselektroden aufgeklebt, ein adäquater i.v.-Zugang gesichert und die notwendigen Reanimationsmedikamente und -infusionen vorbereitet werden. Benutzen Sie Infusionswärmer und Wärmedecken, um eine perioperative Hypothermie zu vermeiden, und überwachen Sie die Patiententemperatur.

Kommt es zu einem Kreislaufstillstand, dann folgen Sie dem ALS-Algorithmus mit geeigneten Modifikationen. Stellen Sie Lage und Höhe des OP-Tisches so ein, das Sie die Thoraxkompressionen optimal durchführen können. Eine Reanimation ist am besten in Rücken-, aber grundsätzlich auch in Bauchlage möglich, wenn eine Umlagerung in Rückenlage nicht sofort umsetzbar ist $[432,433]$. Risikofaktoren für einen Kreislaufstillstand in Bauchlage sind Herzveränderungen der Patienten mit großer Wirbelsäulenchirurgie, Hypovolämie, Luftembolie, Wundspülungen mit Wasserstoffperoxid oder verminderter venöser Rückstrom.

\section{Erkennen der Ursache}

In vielen Fällen eines perioperativen Kreislaufstillstands kommt die physiologische Verschlechterung Schritt für Schritt, die Ursache ist bekannt und der Kreislaufstillstand nicht unerwartet. Ist er doch unerwartet, folgen Sie dem Standard-ABCAlgorithmus, um reversible Ursachen zu finden und zu therapieren. Verschlechtert 
sich der Patient, rufen Sie sofort einen erfahrenen Kollegen zu Hilfe. Informieren Sie das Operationsteam über die Verschlechterung und die Gefahr eines Kreislaufstillstands, und stellen Sie sicher, dass genügend ausgebildetes Assistenzpersonal zur Verfügung steht.

- Katastrophale Blutungen sind in der Regel leicht erkennbar, können aber unentdeckt bleiben, wenn es in Körperhöhlen (Bauchraum, Thorax) oder bei multiplen Frakturen in das Weichteilgewebe blutet. Blutungen ins Becken oder in den Retroperitonealraum können ebenfalls zu einem schnellen Blutverlust führen und müssen ausgeschlossen werden, bei präoperativer Instabilität z. B. durch Ultraschall. In Fällen, in denen eine direkte chirurgische Intervention die Blutung nicht kontrollieren kann, soll frühzeitig eine interventionelle radiologische Therapie in Betracht gezogen werden.

- Ein fehlender Atemwegszugang ist häufig Ursache für einen perioperativen Kreislaufstillstand. Beurteilen Sie vor der Narkoseeinleitung sorgfältig den Atemweg, bereiten Sie die komplette Ausrüstung vor, hierzu zählen auch Absaugmöglichkeiten und ein Operationstisch, der eine Kopftieflage (TrendelenburgPosition) erlaubt. Stellen Sie sicher, dass alles Material für einen schwierigen Atemweg sofort verfügbar und das Team entsprechend vorbereitet ist. Verwenden Sie immer Kapnographie. Kinder sind besonders anfällig für einen Laryngospasmus. Stellen Sie sicher, dass ein geeignetes Muskelrelaxans bereitliegt, und geben Sie dieses, um den Laryngospasmus zu durchbrechen, bevor sich eine signifikante Hypoxie einstellt.

- Ein nicht diagnostizierter Spannungspneumothorax ist ein einfach zu behandelnder Grund für einen Kreislaufstillstand. Obwohl eher im $\mathrm{Zu}$ sammenhang mit einem Trauma üblich, ziehen Sie diesen bei jedem Patienten frühzeitig in Betracht, besonders bei chronisch obstruktiver Lungenerkrankung (COPD) und schwerem Asthma. Ein plötzlicher Anstieg des Beatmungsdruckes kann einen Spannungspneumothorax oder eine Tubusverlegung anzeigen, aber denken Sie auch an Asthma und Anaphylaxie.

- Ein Kollaps kann mehrere Ursachen haben. Im Kontext eines perioperativen Kreislaufstillstands sind die üblichen Ursachen Hypovolämie, Anaphylaxie und vagale Reaktionen. Die transthorakale Echokardiographie ist zum Ausschluss einer Herzbeuteltamponade (falls vermutet) und zur Beurteilung der myokardialen Kontraktilität und Füllung hilfreich.

\section{Anaphylaxie}

Die Inzidenz einer immunvermittelten Anaphylaxie während einer Narkose liegt zwischen $1 / 10.000$ bis $1 / 20.000$. Muskelrelaxantien stellen mit $60 \%$ die häufigste Ursache dar. Die hiermit verbundene Morbidität und Mortalität ist hoch, insbesondere dann, wenn es zu Verzögerungen bei Diagnostik und Therapie kommt. Das initiale Management entspricht dem ABC-Vorgehen, die weiteren Hinweise finden sich im Abschnitt Anaphylaxie. Adrenalin ist das effektivste Medikament bei einer Anaphylaxie und wird so früh wie möglich verabreicht. Vom Anästhesisten soll es i.v. gegeben werden, wenn notwendig, auch mehrfach.

Besteht der Kreislaufstillstand trotz korrekter Behandlung der Anaphylaxie weiter (s. Anaphylaxie), wird die Reanimation unter Beachtung des StandardALS-Algorithmus fortgeführt (s. Erwachsenen-ALS-Kapitel, [168]).

\section{Systemisch-toxische Wirkung von Lokalanästhetika}

Kreislaufstillstand ist eine seltene, aber bekannte Komplikation der Lokalanästhetikaüberdosierung, insbesondere nach unbeabsichtigter intravasaler Injektion. Die direkte Wirkung des Lokalanästhetikums an den kardialen Myozyten verursacht einen Kollaps, üblicherweise bis $5 \mathrm{~min}$ nach der Injektion, aber die Symptome können nach $30 \mathrm{~s}$ bis 60 min einsetzen. Blutdruckabfall, Herzrhythmusstörungen und Krampfanfälle sind typische Manifestationen, wobei hier im Ausschlussverfahren diagnostiziert wird [436].
Die i.v.-Lipidtherapie wird beim Kreislaufkollaps und beim Kreislaufstillstand als Notfalltherapie eingesetzt, ihre Effektivität ist jedoch in der Diskussion [437]. Da keine Hinweise auf eine Schädigung des Patienten dokumentiert sind, empfehlen die Leitlinien die Bevorratung von $20 \%$-Lipidlösung an allen Arbeitsplätzen (Operationsraum, Behandlungszimmer, Notaufnahme), an denen Patienten hohe Dosen von Lokalanästhetika erhalten [353, 438]. Stoppen Sie die Injektion des Lokalanästhetikums, und rufen Sie um Hilfe. Sichern Sie den Atemweg, und intubieren Sie, wenn notwendig. Geben Sie $100 \%$ Sauerstoff, und stellen Sie eine adäquate Ventilation sicher (eine Hyperventilation kann durch einen steigenden Plasma-pH-Wert bei metabolischer Azidose hilfreich sein). Kontrollieren Sie Krampfanfälle mit Benzodiazepin, Thiopental oder Propofol. Geben Sie einen initialen i.v.-Bolus der $20 \%$-Lipidlösung in einer Dosierung von $1,5 \mathrm{ml} / \mathrm{kgKG}$ über $1 \mathrm{~min}$, gefolgt von einer Dauerinfusion von $15 \mathrm{ml} / \mathrm{kgKG} / \mathrm{h}$. Wenn nach 5 min kein ROSC erreicht wurde, verdoppeln Sie die Infusionsgeschwindigkeit und geben maximal zwei weitere Boli im 5-min-Abstand, bis ein ROSC eingetreten ist. Die maximale kummulative Dosis liegt bei $12 \mathrm{ml} / \operatorname{kgKG}[259,260]$.

\section{Diagnose des Kreislaufstillstands}

Asystolie und Kammerflimmern werden direkt bemerkt werden, aber der Beginn einer PEA ist vielleicht nicht so offensichtlich - der Verlust des Pulsoxymetriesignals und sehr niedrige endtidale $\mathrm{CO}_{2}$-Werte sind gute Hinweise, sie sollen zu einer Pulskontrolle führen. Verschwenden Sie keine Zeit mit nicht invasiver Blutdruckmessung.

\section{Therapie des Kreislaufstillstands}

Die Therapie des Kreislaufstillstands folgt den Prinzipien des ALS-Algorithmus. Thoraxkompressionen in Bauchlage funktionieren mit oder ohne sternalen Gegendruck. In einer Studie zur Reanimation in Bauchlage mit sternalem Gegendruck (durch einen Sandsack) konnte hier im Vergleich zur Standardreanimation ein höherer mittlerer arterieller Druck erreicht werden [439]. Ziehen Sie eine direkte offene Herz- 
massage in Betracht, wenn der Thorax eröffnet oder das Herz einfach zugänglich ist.

\section{Kammerflimmern}

Liegt Kammerflimmern vor, rufen Sie nach einem Defibrillator. Ist dieser nicht direkt verfügbar, verabreichen Sie einen präkordialen Faustschlag. Ist dieser nicht erfolgreich, starten Sie Thoraxkompressionen und Beatmung, bis der Defibrillator eintrifft. Suchen Sie sofort nach reversiblen Ursachen: Hypoxie und Hypovolämie sind in dieser Situation die häufigsten.

\section{Asystolie/extreme Bradykardie}

Beenden Sie sofort jegliche chirurgische Intervention, die eine vagale Reaktion hervorrufen kann. Wenn diese anzunehmen ist, geben Sie 0,5 mg Atropin i.v./i.o. (nicht $3 \mathrm{mg}$ ). Beginnen Sie mit der Reanimation, und suchen Sie sofort nach anderen reversiblen Ursachen. Schließen Sie bei einem 0-Linien-EKG ein loses EKG-Kabel aus.

\section{Pulslose elektrische Aktivität}

Beginnen Sie die Reanimation, während Sie schnell nach reversiblen Ursachen für die PEA suchen. Geben Sie Volumen, bis Sie sicher sind, dass das intravasale Volumen adäquat ist. Stoppen Sie die Narkosemittel. Wird ein Vasopressor benötigt, ist unter diesen Umständen $1 \mathrm{mg}$ Adrenalin (wie in den StandardALS-Leitlinien) möglichweise zu viel. Geben Sie eine geringere Dosis $(1 \mu \mathrm{g} /$ $\mathrm{kgKG}$ ) Adrenalin oder anfangs einen anderen Vasopressor; führt dieses Vorgehen nicht zu kardialem Output, erhöhen Sie die Dosis, während Sie mit Thoraxkompressionen und Beatmung fortfahren.

\section{Monitoring und Feedback während der Reanimation}

Während bei präklinischem Kreislaufstillstand die Monitorüberwachung oft eingeschränkt ist, können Patienten im perioperativen Umfeld mit höherer Präzision überwacht werden.

Monitoring ermöglicht es, die Leistung der Helfer und die Reaktion des Patienten zu beurteilen.

- CPR-Qualität
Feedbacksensoren (z. B. Accelerometer) sorgen für effektive Thoraxkompressionen und ermöglichen dem Anwender, seine Leistung direkt anzupassen. Vorhandene Systeme sind unbedingt zu nutzen. Leistungsfeedback kann auch von invasivem und nicht-invasivem Patientenmonitoring abgelesen werden; der Anwender soll direkten Blick auf den Monitor haben.

- Patientenreaktion

Patientenüberwachung und -beurteilung erfordert adäquate Lichtverhältnisse und Abdecken des Patienten. Nicht-invasive Blutdruckmessung hilft bis zum Erreichen von ROSC nicht weiter, bei invasiver Blutdrucküberwachung sollen diastolische Werte von $>25 \mathrm{mmHg}$ erreicht werden. Titrieren Sie bei optimaler Thoraxkompression einen Vasopressor so, dass Sie diesen Zielbereich erreichen. Dieser Zielwert basiert auf Expertenmeinung, die von experimentellen und wenigen klinischen Daten abgeleitet ist [441-443].

Kapnographie ist während der Narkose ein Minimalstandard und steht dementsprechend bei perioperativem Kreislaufstillstand sofort zur Verfügung. Ergänzend zum Einsatz bei Patienten mit endotrachealer Intubation, bei denen siev. a. zur Beurteilung der sicheren Tubuslage wertvoll ist, kann die Kapnographie auch bei supraglottischen Atemwegsinstrumenten genutzt werden (obwohl eine Undichtigkeit die quantitative Beurteilung erschweren kann). Ein endtitaler $\mathrm{CO}_{2}$ Wert $\left(\mathrm{EtCO}_{2}\right)$ von $<1,4 \mathrm{kPa} / 10 \mathrm{mmHg}$ weist auf einen niedrigen kardialen Output hin, und die Helfer müssen ihre Technik optimieren, um höhere Werte zu erreichen. Ein plötzlicher Anstieg auf normale $(4,7-5,4 \mathrm{kPa} / 35-40 \mathrm{mmHg})$ oder noch höhere Werte kann ein Indikator für ROSC sein. Passen Sie Ihre Reanimationsmaßnahmen so an, dass bei Beatmung mit 10/min und einem Zugvolumen, durch das sich der Thorax leicht hebt, ein $\mathrm{EtCO}_{2}>2,7 \mathrm{kPa} / 20 \mathrm{mmHg}$ erreicht wird [440].

\section{Teamarbeit}

Jede Reanimation soll von einem Teamleader geführt werden, der alle eingesetzten Kräfte und Reanimations- maßnahmen mit dem Schwerpunkt auf qualitativ hochwertige Reanimation anweist und koordiniert. Beenden Sie die operativen Maßnahmen, außer wenn sich diese auf eine reversible Ursache richten. Der Zugang und die Reanimationsmaßnahmen erfordern es u. U., dass das OP-Gebiet abgedeckt wird und das OPTeam sich vom Patienten zurückzieht. Priorisieren Sie die Teammaßnahmen, stellen Sie gute BLS-Qualität (Basisreanimation) sicher, identifizieren Sie reversible Ursachen, und vermeiden Sie unnötige Maßnahmen. Reagiert der Patient nicht auf Ihre Reanimationsbemühungen (z. B. $\mathrm{EtCO}_{2}<2,7 \mathrm{kPa} / 20 \mathrm{mmHg}$ ), versuchen Sie, die Qualität der Reanimation zu optimieren: Kompressionszeitanteil, Kompressionsfrequenz, Kompressionstiefe, Entlastung und Vermeidung von Hyperventilation [440].

\section{Postreanimationsbehandlung}

Abhängig von den Umständen brauchen Patienten nach nur kurzer Reanimationsdauer (z. B. nach Asystolie bei exzessiver vagaler Reizung) nur die normale postoperative Überwachung. Alle Patienten mit längerer Reanimationsdauer benötigen eine Intensivtherapie, es sei denn, eine weitere aktive Behandlung erscheint nicht angebracht. In den meisten Fällen werden bis auf lebensrettende operative alle weiteren Maßnahmen abgebrochen, um eine Aufnahme auf eine Intensivstation zur Postreanimationsbehandlung zu ermöglichen. Patienten, die erst nach einer längeren Zeit erfolgreich reanimiert werden konnten, können eine systemisch-inflammatorische Reaktion (SIRS) mit dem Risiko eines Multiorganversagens entwickeln. Sie benötigen eine Optimierung des mittleren arteriellen Blutdruckes, der Ventilation und der Oxygenierung. Diese Patienten können einen signifikanten zerebralen Insult erlitten haben. Einige von ihnen kommen möglicherweise für ein zielgerichtetes Temperaturmanagement infrage, was sorgfältig überlegt werden soll, da im Umfeld der perioperativen Reanimation keine Daten für diese Therapie vorliegen. Aktive Blutungen sind sicher eine Kontraindikation für milde Hypothermie, aber Fieber soll in allen Fällen vermieden werden. Das Verhindern einer Hyper- 
thermie, bedingt durch Überwärmung oder das Post-cardiac-arrest-Syndrom, ist wichtig, um die neurologische Erholung zu optimieren [444].

\section{Do-not-attempt-resuscitation- (DNAR-)Entscheidungen}

Patienten mit DNAR-Verfügung, die sich zur Operation vorstellen, bringen den Anästhesisten in ein Dilemma. Die Anästhesie verursacht eine kardiale Instabilität, viele der Routinemaßnahmen, die zur Anwendung kommen, können als Reanimationsmaßnahmen gewertet werden, aber die Überlebenswahrscheinlichkeiten eines intraoperativen Kreislaufstillstands sind besser als bei einem innerklinischen insgesamt. Bedenken und diskutieren Sie jeden Einzelfall mit Blick auf die Erfolgsaussichten mit dem Patienten und/oder seinen Angehörigen. Einige Patienten möchten ungeachtet dessen die DNAR-Verfügung aufrechterhalten, andere wollen sie temporär aussetzen. Vereinbaren Sie den Zeitpunkt, ab wann die DNAR-Order wieder Gültigkeit haben soll [445].

\section{Kreislaufstillstand in der Herzchirurgie}

\section{Einleitung}

In der unmittelbar postoperativen Phase nach größeren herzchirurgischen Eingriffen ist ein Kreislaufstillstand recht üblich, die Inzidenz liegt bei $0,7-8,0 \%$ [446-455]. Üblicherweise geht eine physiologische Verschlechterung voraus [456], er kann aber auch plötzlich bei stabilen Patienten auftreten [452]. Es gibt üblicherweise einen konkreten Grund für den Kreislaufstillstand, z. B. Herzbeuteltamponade, Hypovolämie, myokardiale Ischämie, Spannungspneumothorax oder Schrittmacherversagen. Diese Gründe sind alle reversibel, und wenn sie prompt behandelt werden, hat der Kreislaufstillstand nach Herzchirurgie eine vergleichsweise hohe Überlebensrate. Der Schlüssel zur erfolgreichen Reanimation dieser Patienten ist es, rechtzeitig die Notwendigkeit der Resternotomie zu erkennen, speziell bei Herzbeuteltamponade oder Blutung, also in Situationen, in denen Thoraxkompressionen ineffektiv sein können.

\section{Beginn der CPR}

Wird VF oder eine Asystolie diagnostiziert, defibrillieren Sie sofort, oder stimulieren Sie mit einem externen Schrittmacher mit maximaler Amplitude. Bei Patienten mit Kreislaufstillstand, bei denen das Monitoring fehlenden Auswurf anzeigt, beginnen Sie sofort mit Thoraxkompressionen. Sichern Sie die Effektivität der Kompressionen durch Blick auf die arterielle Druckkurve, bemühen Sie sich, einen systolischen Blutdruck von > $60 \mathrm{mmHg}$ ["Society of Thoracic Surgeons (STS) Clinical Practice Guidelines“ in Vorbereitung - persönliche Mitteilung von Joel Dunning] und einen diastolischen Druck von $>25$ mmHg mit einer Frequenz von 100$120 /$ min zu erreichen [440]. Gelingt dies mit externer Thoraxkompression nicht, ist eine Herzbeuteltamponade oder extremer Volumenmangel wahrscheinlich, und es soll resternotomiert werden.

Denken Sie an andere reversible Ursachen:

- Hypoxie - prüfen Sie die Tubuslage, beatmen Sie mit 100\% Sauerstoff.

- Spannungspneumothorax - prüfen Sie die Lage der Trachea, auskultieren Sie.

- Schrittmacherversagen - prüfen Sie den Schrittmacherimpuls und die Unversehrtheit der Elektroden. Bei Asystolie durch Schrittmacherversagen können die Thoraxkompressionen momentan aufgeschoben werden, wenn die implantierten Schrittmacherdrähte schnell wieder angeschlossen und die Schrittmacherfunktionen wiederhergestellt werden können (DDD mit 100/min mit maximaler Amplitude).

\section{Defibrillation}

Es gibt Bedenken, ob externe Thoraxkompressionen zu einer Ruptur der Sternumnaht oder kardialen Verletzungen führen können [457-460]. Auf der kardiochirurgischen Intensivstation soll ein am Monitor beobachteter VF/ pVT-Kreislaufstillstand deshalb sofort mit bis zu drei schnellen sukzessiven Defibrillationsversuchen behandelt werden. Drei vergebliche Schocks nach herzchirurgischem Eingriff sind der Trigger für die Notwendigkeit der notfallmäßigen
Resternotomie. Die weiteren Defibrillationen folgen dem universellen Algorithmus; falls der Patient resternotomiert ist, mit internen Paddles und 20 J [461, 462].

\section{Notfallmedikamente}

Titrieren Sie Adrenalin sehr vorsichtig bis zum gewünschten Effekt (i.v.-Dosen bis $100 \mathrm{mcg}$ bei Erwachsenen). Bei Patienten mit refraktären schockbaren Rhythmen (VF/pVT) erwägen Sie Amiodaron $300 \mathrm{mg}$, verzögern Sie dabei aber nicht die Resternotomie. Atropin ist bei Asystolie nicht empfehlenswert, dafür soll temporäres oder externes Pacing eingesetzt werden.

\section{Notfallresternotomie}

Wenn alle anderen reversiblen Ursachen ausgeschlossen sind, ist die Resternotomie ein integraler Bestandteil der Reanimation nach Kardiochirurgie. Ist der Atemweg adäquat gesichert, die Beatmung wurde begonnen und drei Defibrillationsversuche bei VF/pVT waren ohne Erfolg, soll die Resternotomie ohne Verzögerung erfolgen. Wenn andere Maßnahmen versagt haben, ist sie auch bei Asystolie oder PEA indiziert und soll innerhalb von 5 min nach dem Kreislaufstillstand durch jemanden mit entsprechender Ausbildung durchgeführt werden.

Diese Leitlinien sind auch für Patienten geeignet, die eine minimal-invasive Kardiochirurgie ohne Sternotomie hatten. Chirurgen, die solche Operationen durchführen, müssen Vorbereitungen zur Rethorakotomie im Fall eines Kreislaufstillstands getroffen haben.

Die speziellen Bedingungen, die für die Behandlung von Patienten mit mechanischen Herzunterstützungssystemen („ventricular assist devices“, VAD) gelten, sind im Kapitel über spezielle Patienten besprochen (s. „Patienten mit mechanischen Herzunterstützungssystemen").

\section{Kreislaufstillstand im Herzkatheterlabor}

Ein Kreislaufstillstand kann sich während einer perkutanen Koronarintervention (PCI) bei ST-Hebungsinfarkt (STEMI) oder Nicht-Hebungsinfarkt (non-STEMI) ereignen. Gleichfalls kann der Kreislaufstillstand eine Komplikation einer Angio- 
graphie sein, wie z. B. Katheterblockade, Luft- oder Thrombembolie der Koronargefäße, Dissektion der Koronarintima durch die Spitze des Angiographiekatheters oder Perikardtamponade durch eine perforierte Koronararterie. Die meisten Komplikationen werden in einem VF mit der sofort notwendigen Defibrillation enden. Deswegen sollen Patienten kontinuierlich monitorisiert sein, und ein Defibrillator muss im Angiographieraum zur Verfügung stehen. Selbstklebende, röntgendurchlässige Defibrillationselektroden können bei Hochrisikopatienten vor Beginn des Eingriffs angebracht werden.

In diesem speziellen Umfeld und mit der Möglichkeit, umgehend auf ein monitorisiertes VF zu reagieren, wird die sofortige Defibrillation ohne vorausgehende Thoraxkompressionen empfohlen. Wenn ein Patient in der frühen elektrischen Phase eines Kreislaufstillstands ist, kann eine erfolgreiche Defibrillation (VF-Ende und ROSC), im Gegensatz zu den Aussagen in den Leitlinien für Kreislaufstillstände ohne Überwachung und OHCA, erreicht werden, bevor Thoraxkompressionen gestartet wurden. Bei fehlgeschlagener Defibrillation oder wenn das VF sofort wiederkehrt, soll umgehend noch bis zu 2-mal defibrilliert werden.

Wenn das VF nach den drei initialen Defibrillationen weiterbesteht oder ROSC nicht mit Sicherheit erzielt wurde, beginnen Sie ohne weiteren Verzug mit Thoraxkompressionen und Beatmung. Die Ursache des ungelösten Problems muss weiter mit der Koronarangiographie gesucht werden. Es ist extrem wichtig, dass die Thoraxkompressionen für die Angiographie nicht unterbrochen werden. Auf dem Angiographietisch, mit dem Bildverstärker über dem Patienten, sind Thoraxkompressionen von adäquater Tiefe und Frequenz fast unmöglich. Zudem sind die Helfer gefährlicher Strahlung ausgesetzt. Deswegen ist der frühe Wechsel zum Einsatz einer mechanischen Reanimationshilfe dringend empfohlen [247, 463]. Wenn das zugrunde liegende Problem nicht schnell behoben werden kann und die Infrastruktur zur Verfügung steht, gibt es eine geringe Evidenz dafür, dass extrakorporale Unterstützungsverfahren
(ECLS) im Sinne einer Rescue-Strategie erwogen werden können. Extrakorporale Verfahren sind möglicherweise der intraaortalen Ballonpumpe (IABP) vorzuziehen [464]. Es gibt keine Evidenz, die Impellerpumpe allein zur Kreislaufunterstützung während eines Kreislaufstillstands zu empfehlen.

Wenn der Kreislaufstillstand durch einen nicht schockbaren Rhythmus verursacht wurde, soll eine rasch durchgeführte transthorakale Echokardiographie einen Perikarderguss finden oder andere Ursachen identifizieren können.

\section{Kreislaufstillstand auf der Dialysestation}

\section{Einführung}

Der plötzliche Herztod ist die häufigste Todesursache von Hämodialysepatienten; normalerweise gehen ventrikuläre Arrhythmien voraus [465]. Hyperkaliämie ist in 2-5\% ursächlich für den Tod von Hämodialysepatienten [466] und führt in bis zu $24 \% \mathrm{zu}$ einer Notfalldialyse [467]. Wohl durch die größere Flüssigkeits- und Elektrolytimbalance nach dem Wochenendintervall ist die Häufigkeit für einen Kreislaufstillstand bei der ersten Hämodialyse in der Woche (z. B. Montag oder Dienstag) am höchsten [468]. Die Prävention eines Kreislaufstillstands bei Dialysepatienten besteht in der Vermeidung von Dialyselösungen mit niedrigem Kaliumgehalt und sorgfältiger Medikation, z. B. von Beta-Blockern oder Angiotensin-Converting-Enzym- (ACE-) Hemmern [465]. Es gibt wenig Evidenz für eine Empfehlung zur Behandlung von Kreislaufstillstand während Hämodialyse, trotzdem gibt es einige spezielle Vorschläge [469].

\section{Erste Schritte}

- Alarmieren Sie sofort das Reanimationsteam, und fordern Sie Expertenhilfe an.

- Nutzen Sie den universellen Algorithmus.

- Bestimmen Sie eine ausgebildete Dialysefachperson, die das Dialysegerät bedient.

- Stoppen Sie die Filtration (z. B. Flüssigkeitsentzug), und geben Sie einen Flüssigkeitsbolus.
- Geben Sie das Patientenblut aus dem Dialysegerät zurück und

- Hängen Sie das Gerät ab.

- Halten Sie die Dialysezugänge offen, und nutzen Sie sie zur Medikamentengabe.

- Vorsicht wegen feuchter Oberflächen (z. B. Leck am Dialysegerät).

- Minimieren Sie Verzögerungen bei der Defibrillation.

\section{Modifikationen bei der Herz- Lungen-Wiederbelebung}

\section{Defibrillation}

Hämodialysepatienten haben häufiger einen schockbaren Rhythmus [247, 463] als der Rest der Bevölkerung [247, 463]. Um die sicherste Methode der Defibrillation während Dialyse festzulegen, braucht es weitere Studien. Die meisten Hersteller von Dialysegeräten empfehlen das Abhängen des Geräts vor der Defibrillation [474]. Vergewissern Sie sich über die verwendeten Dialysegeräte, und prüfen Sie, ob Ihres ein „Für Defibrillation geeignet“Label entsprechend des InternationalElectrotechnical-Committee- (IEC-) Standards aufweist. Automatisierte externe Defibrillatoren können in einem pflegegeführten Dialysezentrum die frühe Defibrillation durch Ersthelfer mit geeigneter Ausbildung unterstützen [475].

\section{Venenzugang}

Nutzen Sie in lebensbedrohenden Situationen und beim Kreislaufstillstand die Dialysezugänge [469].

\section{Mögliche reversible Ursachen}

Alle üblichen reversiblen Ursachen (4 Hs und HITS) gelten auch für Dialysepatienten. Elektrolytstörungen, besonders Hyperkaliämie (s. „Hypo-/Hyperkaliämie und Elektrolytstörungen") und Flüssigkeitsüberladung (z. B. Lungenödem) sind häufige Ursachen.

\section{Versorgung nach erfolgreicher Wiederbelebung}

In der frühen Phase nach Reanimation kann eine Dialyse, je nach Volumenstatus und Serumbiochemie, notwendig sein. Der Transfer des Patienten in eine Einrichtung mit Dialysemöglichkeit (z. B. Intensiv- oder Dialysestation) ist notwendig. 


\section{Kreislaufstillstand in der Zahnarztpraxis}

\section{Einführung}

Zahnärztliche Notfälle beinhalten eine Vielzahl von Situationen. Diese reichen von psychosomatischen Störungen, ausgelöst durch Angst, bis hin zu lebensbedrohenden Situationen, die eine sofortige notfallmedizinische Versorgung notwendig machen. Kreislaufstillstände in der Zahnarztpraxis sind mit einer Inzidenz von 0,002-0,011 Fällen pro Zahnarzt und Jahr selten [476-478].

Die häufigsten medizinischen Notfälle sind vasovagale (Prä-)Synkopen, orthostatische Hypotension, hypertensive Krisen, Hyperventilation, Krampfanfälle, moderate allergische Reaktionen, Hypoglykämie und Anginaanfälle [476, 479]. Die Mehrheit der Zahnärzte geben an, dass sie in der Lage sind, die intitiale Behandlung der üblichen Notfälle zu gewährleisten, während viele sich nicht in der Lage fühlen, eine Anaphylaxie, einen Myokardinfarkt oder einen Kreislaufstillstand zu behandeln [476, 477].

Der Kreislaufstillstand in einer Zahnarztpraxis ist ein durch medizinisches Personal beobachtetes Ereignis. Das Personal hat die Pflicht zur Versorgung und muss in der Lage sein, CPR anzuwenden.

\section{Gründe für den Kreislaufstill- stand}

Gründe für einen Kreislaufstillstand sind für gewöhnlich in vorexistierenden Erkrankungen oder Komplikationen der durchgeführten Behandlung begründet. Die lebensbedrohenden Notfälle haben üblicherweise ihre Ursache im Myokardinfarkt, generalisierten Krampfanfällen („grand mal“) oder exazerbiertem Asthma. Zahnärztliche Behandlungen können durch die zugrunde liegende Pathologie oder durch Komplikationen (z. B. Blutung, Sekrete, Gewebeschwellung) zum Verlust des Atemwegs führen. Mit einer berichteten Inzidenz von 0,07-0,09 Fällen pro Zahnarzt und Jahr ist Ersticken selten [476, 477]. Zusätzliche Sedierung erhöht das Risiko für diese Fälle, obwohl die zahnärztliche Behandlung mit Lokalanästhesie und Sedierung oder mit einer Kombination aus beidem einen exzellenten Sicherheitsstandard hat [480, 481].

Obwohl eine lebensbedrohende Anaphylaxie selten ist, ist sie ein dokumentierter Grund für das Versterben während einer zahnärztlichen Behandlung. Zusätzlich zu ChlorhexidinMundspülungen können Penicillin und Latex andere Auslöser sein. Anaphylaxie auf Lokalanästhetika ist selten und meist Ursache einer direkten intravasalen Injektion einer mit Adrenalin versetzten Lösung. Wirkliche Anaphylaxie (alle Ursachen) kommt nur in 0,0040,013 Fällen pro Zahnarzt pro Jahr vor. $\mathrm{Zu}$ einem Koronarsyndrom (Angina pectoris oder Myokardinfarkt) kommt es hingegen in 0,15-0,18 Fällen pro Jahr $[476,477]$.

\section{Behandlung des Kreislaufstillstands}

Die folgenden Modifikationen für die Initialbehandlung des Kreislaufstillstands in einem Zahnarztstuhl sind empfohlen:

- Wenn es zum plötzlichen Bewusstseinsverlust kommt, holen Sie sofort Hilfe.

- Inspizieren Sie den Mund der betroffenen Person. Alles feste Material aus der Mundhöhle entfernen (z. B. Retraktor, Sauger, Tampons usw.). Um einer Atemwegsobstruktion vorzubeugen, soll der Patient in Rückenlage gebracht werden.

- Bringen Sie den Zahnarztstuhl in die Horizontale. Der Herzauswurf kann wiederhergestellt werden, wenn ein reduzierter venöser Rückfluss oder eine Vasodilatation, z. B. durch eine vagale Synkope oder eine orthostatische Hypotension, den Bewusstseinsverlust ausgelöst hat. Bei diesen Patienten kann es auch helfen, die Beine anzuheben oder den Zahnarztstuhl in Kopftieflage zu bringen.

- Öffnen Sie gleichzeitig den Atemweg, und prüfen Sie die Atmung (sehen, hören, fühlen). Wenn die Atmung nicht normal oder nicht vorhanden ist, gehen Sie bis zum Beweis des Gegenteils von einem Kreislaufstillstand aus. Wenn verfügbar, beauftragen Sie jemanden, einen AED zu holen.

- Einige Fallberichte beschreiben eine erfolgreiche CPR im Zahnarztstuhl [482, 483]. Nur wenige Simulations- untersuchungen vergleichen die Effektivität der CPR im Zahnarztstuhl mit CPR auf dem Boden und zeigen eine entweder schlechtere oder allenfalls gleichwertige CPR-Qualität bei Reanimation im Zahnarztstuhl [484487]. Wie auch immer, der Patient soll aufgrund des Verletzungsrisikos für ihn und die Helfer sowie der limitierten Platzverhältnisse auf dem Boden nicht vom Zahnarztstuhl gehoben werden [482, 483]. Stellen Sie sicher, dass der Zahnarztstuhl in Horizontalposition ist, das Kopfteil kann mit einem Hocker für zusätzliche Stabilität unterstützt werden. Dann starten Sie unverzüglich die Thoraxkompressionen [482, 484].

- Wenn CPR-Feedback-Geräte für die Überprüfung der CPR-Qualität benutzt werden, können diese Geräte, die mit Beschleunigungssensoren arbeiten, auf einem Zahnarztstuhl die Kompressionstiefe überschätzen [488].

- Für Erwachsene und Kinder gilt das Standard-KompressionsVentilations-Verhältnis. Erwägen Sie die Überkopftechnik für die CPR, wenn der Zugang zum Thorax auf einer Seite limitiert ist [489-492].

- Halten Sie den Atemweg offen, und beatmen Sie den Patienten mit Beutel und Maske. Wenn nötig, wenden Sie dabei die 2-Hand-Technik an. Wenn der Anwender geübt ist, können supraglottische Atemwegshilfen eingesetzt werden. Die tracheale Intubation ist nicht empfohlen, wird vom Zahnarzt nicht verlangt und soll vermieden werden.

- Schalten Sie den AED ein, und folgen Sie den Anweisungen. Wenn indiziert, geben Sie so bald wie möglich die erste Defibrillation ab.

- Führen Sie die CPR weiter, bis Lebenszeichen vorhanden sind oder der Patient dem professionellen Reanimationsteam übergeben werden kann (s. „Basismaßnahmen der Reanimation beim Erwachsenen und automatisierte externe Defibrillation“, [493]). 


\section{Ausrüstung und Schulung}

Folgen Sie für die Behandlung medizinischer Notfälle in Zahnarztpraxen den Empfehlungen der nationalen Leitlinien für die Ausrüstung [478]. Die Basisausrüstung zur Reanimation soll in allen Zahnarztpraxen mit Primärversorgung sofort verfügbar sein. Dazu zählen Absaugung, Beatmungsbeutel mit Maske, Sauerstoff und Notfallmedikamente [494, 495]. Damit die immer noch unbefriedigende Verfügbarkeit von AED in Zahnarztpraxen zunimmt, soll die Rolle der frühen Defibrillation hervorgehoben werden $[482,496]$. Während in den USA über eine Verfügbarkeit von $11 \%$ berichtet wird, ist sie für Europa 0,5-2,6\% [499]. Wir empfehlen, dass alle Zahnarztpraxen sofortigen Zugriff auf einen AED haben und dass das Personal in der Anwendung geschult ist. Zusätzliches Equipment und spezielle Schulungen sind notwendig, wenn in der Zahnarztpraxis Analgetika und Sedativa zum Einsatz kommen $[478,500]$. Wenn bei Patienten mit Schrittmacher elektrische Geräte (z. B. Diathermie, elektrische Pulpatester usw.) zum Einsatz kommen, sind ein EKG-Monitoring und die sofortige Verfügbarkeit eines Defibrillators empfohlen [482].

Die Öffentlichkeit erwartet zu Recht von Zahnärzten und dem gesamten in der Zahnarztpraxis tätigen Personal, dass es die Kompetenz besitzen, einen Kreislaufstillstand zu behandeln. Leider haben nur 0,2-0,3 \% der Zahnärzte tatsächlich Erfahrung damit $[476,479,501]$. Die Ausbildung variiert dabei zudem signifikant von Land zu Land [476, 477, 501-503]. Die Kenntnisse und Kompetenzen in der Behandlung von medizinischen Notfällen aufrechtzuerhalten muss einen wichtigen Teil der Ausbildung der Zahnärzte ausmachen. Alle professionellen zahnärztlichen Mitarbeiter sollen jährlich ein praktisches Training zum Erkennen und Behandeln von medizinischen Notfällen und in CPR mit einer grundlegenden Ausbildung in Atemwegsmanagement und Gebrauch eines AED durchlaufen [478].
Kreislaufstillstand in

Transportfahrzeugen

\section{An-Bord-Notfälle im Flugzeug während des Fluges}

\section{Einführung}

Weltweit reisen jährlich 3,2 Mrd. Passagiere mit kommerziellen Fluglinien. Dabei kommt es zu einem medizinischen Notfall pro 10.00040.000 Passagiere [504, 505]. Nach 24 Interkontinentalflügen beträgt die Wahrscheinlichkeit für wenigstens einen medizinischen Notfall 95\% [505]. Meistens sind Menschen mittleren Alters betroffen [506]. Zwei kürzlich publizierte große Studien berichten von 22.000 Onboard-Notfällen bei fünf amerikanischen und zwei europäischen Fluggesellschaften. Mit einigen Abweichungen zwischen den verschiedenen Fluggesellschaften betreffen die häufigsten medizinischen Probleme Synkopen oder Präsynkopen $(37,4-53,5 \%)$, respiratorische Symptome (12,1\%), gastrointestinale Probleme $(8,9-9,5 \%)$ und kardiale Erkrankungen (4,9-7,7\%, [504, 507]). Chirurgische Probleme (z. B. tiefe Beinvenenthrombose, Appendizitis, gastrointestinale Blutungen) waren selten $(<0,5 \%$, [504]). Eine Arbeitsunfähigkeit der Crew während des Fluges kommt nicht oft vor, der häufigste Grund war dabei eine akute Myokardischämie [508].

Die Versorgung von medizinischen Notfällen während eines Fluges ist nur eingeschränkt möglich, die meisten Notfälle können aber konservativ mit Flüssigkeitsgabe, Sauerstoff und den anderen Möglichkeiten, die das Erste-Hilfe-Kit im Flugzeug vorhält, gehandhabt werden. Etwa ein Viertel dieser Patienten braucht eine weitere Abklärung im Krankenhaus [507]. Eine notfallmäßige Umleitung eines Flugzeugs wird lediglich in 2,4-7,3\% aller Ereignisse notwendig, meist dann, wenn Thoraxschmerzen, der Verdacht auf einen Schlaganfall oder zerebrale Krampfanfälle vorliegen [504, 507, 509, 510].

Ein Kreislaufstillstand hat eine Inzidenz von einem Ereignis bei 5-10 Mio. Passagierflügen. Ein initial defibrillierbarer Rhythmus liegt dabei in 25-31\% vor [505, 511-513], der Einsatz eines AED hat dann eine Überlebens- rate bis zur Krankenhausaufnahme von 33-50\% Faktoren [511, 513, 514], die zu einer hohen Überlebensrate beitragen, sind das beobachtete Ereignis, die in BLS ausgebildeten Flugbegleiter und medizinisches Fachpersonal, das in 73-86\% der Fälle im Flugzeug mitreist und sofort Hilfe leisten kann [504, 507, 509]. Trotzdem versterben in Flugzeugen der International Airlines Transport Association (IATA) ca. 1000 Personen/ Jahr. Einige Studien haben gezeigt, dass 41-59\% der Kreislaufstillstände an Bord eines Flugzeugs unbeobachtet sind und während des Schlafens erfolgen. Wenn der initiale Rhythmus eine Asystolie oder ein idioventrikulärer Rhythmus war, gab es dabei keine Überlebenden $[511,513]$.

\section{Herz-Lungen-Wiederbelebung im Flugzeug}

Im Fall eines Kreislaufstillstands wenden Sie den universellen BLS-Algorithmus an (s. „Basic Life Support bei Erwachsenen und automatisierte Defibrillation“, [493]). Fordern Sie sofort einen AED und das Erste-Hilfe-Kit von den Flugbegleitern an. Ärzte und geschultes medizinisches Personal, z. B. Pflege- oder Rettungsdienstpersonal, sollen nach erweitertem medizinischem Equipment fragen. Entsprechend den Kompetenzen und der vorhandenen medizinischen Ausrüstung wird der Patient mit den erweiterten lebensrettenden Maßnahmen versorgt, dabei muss sichergestellt sein, dass die CPR mit hoher Qualität weiterläuft und der AED korrekt angebracht wurde (s. „Erweiterte lebensrettende Maßnahmen beim Erwachsenen “, [168]).

Erwägen Sie die folgenden Modifikationen bei der CPR:

- Stellen Sie sich selbst und Ihre Berufsqualifikation der Kabinencrew vor.

- Aufgrund der engen Platzverhältnisse im Gang eines Flugzeugs ist die Ausführung der CPR limitiert. Der Patient soll sofort an einen geeigneteren Platz (z. B. in einen Durchgang oder den Ausgangsbereich) gebracht werden. Wenn der Zugang zum Patienten keine konventionelle $\mathrm{CPR}$ zulässt, erwägen Sie die Überkopfmethode [489-492]. 
- Schließen Sie während der CPR Sauerstoff an der Gesichtsmaske oder am Beatmungsbeutel an.

- Ersuchen Sie um eine Flugumleitung zum nächstgelegenen geeigneten Flugplatz. Legen Sie bei anderen, nicht kritischen Notfällen, mit der Flugcrew eine optimale Versorgungsstrategie fest. Die Erwägung einer Flugumleitung hängt vom Zustand des Patienten und der notwendigen dringenden Krankenhausbehandlung, wie z. B. beim akuten Koronarsyndrom, Schlaganfall, anhaltend verändertem Bewusstsein, aber auch von technischen und operationellen Faktoren ab.

- Erfragen Sie bei der Kabinencrew, ob die Möglichkeit einer medizinischen Konsultation (z. B. durch Sprechfunk oder Satellitentelefon) besteht $[506,510]$.

- Um den Herzrhythmus zu überwachen, kann ein AED mit Monitor bei einem Patienten ohne Kreislaufstillstand (z. B. mit Synkope, Thoraxschmerzen oder Arrhythmien) ohne Gefahr angebracht werden $[507,512$, 513].

- Wenn mitreisende Ärzte zur Hilfe aufgefordert werden, können Bedenken über rechtliche Verantwortlichkeiten entstehen, basierend auf den ethischen Pflichten ist jeder Arzt aufgefordert, seine Hilfe entsprechend seiner Spezialisierung anzubieten, gesetzlich festgelegt ist die Pflicht aber nur in bestimmten Ländern. Gleichwohl führt der sog. Gute-Samariter-Akt und andere Bestimmungen, die vom Herkunftsland des Flugzeugs abhängen, immer da$\mathrm{zu}$, dass Helfer an Bord eines Flugzeugs vor möglichen juristischen Konsequenzen geschützt sind [504, 515].

- Die Todesfeststellung an Bord eines Flugzeugs kann rechtlich nur durch einen Arzt vorgenommen werden. Wenn eine Person tot aufgefunden oder CPR-Maßnahmen beendet wurden, ist eine Flugumleitung nicht empfohlen (s. „Ethik bei der Reanimation und Entscheidungen am Lebensende“, [243]).

\section{Ausbildung und Equipment}

\section{Ausbildung des Flugpersonals}

Sowohl Piloten als auch Flugbegleiter müssen eine initiale und sich periodisch wiederholende Ausbildung für medizinische Notfallmaßnahmen und das dafür notwendige Equipment, inklusive AED und Erste-Hilfe-Kit, erhalten. Lokale Vorschriften können ebenso angewandt werden [516].

Obwohl die zivile Luftfahrt durch eine Vielzahl von nationalen und internationalen Gesetzen geregelt ist, zeigen manche Studien, dass die meisten Notfälle während des Fluges nicht oder uneinheitlich berichtet werden [504, 517]. Die Dokumentation von Notfällen während des Fluges braucht eine Standardisierung mit dem Ziel, die Ausbildung des Kabinenpersonals und die Selektion von Passagiergruppen vor dem Flug zu verbessern.

\section{Notfallequipment in Flugzeugen}

Die „Federal Aviation Administration“ (FAA) verlangt von jedem in den USA kommerziell registrierten Flugzeug mit einer Ladekapazität von mehr als 7500 Pfund und mindestens einem Flugbegleiter, dass ein AED, i.v.-Medikamente und erweitertes Notfallequipment vorhanden ist [518]. In Europa sind diese Vorschriften weniger präzise [519]. In jedem in Europa kommerziell registrierten Flugzeug muss ein ErsteHilfe-Kit, mit dem alle Crew-Mitglieder umgehen können, vorhanden sein. Flugzeuge mit mindestens 30 Sitzplätzen müssen zudem ein erweitertes medizinischen Equipment, das von einer ausgebildeten Person bedient werden kann, mitführen. Trotzdem variiert die Ausstattung signifikant und wird vielleicht für alle Notfälle nicht adäquat sein, jedoch für die meisten Basismaßnahmen ausreichen [504, 517, 520]. Die Mehrzahl der großen europäischen Luftfahrtgesellschaften führt AED mit, bei manchen aber ist ein automatisierter externer Defibrillator nur bei Interkontinentalflügen vorhanden, manche haben sogar gar kein CPR-Equipment an Bord [517].

Basierend auf den Outcomedaten für Überlebende nach Kreislaufstillstand und vor dem Hintergrund, dass es keine Alternative zur Behandlung schockbarer Rhythmen an Bord gibt, empfehlen wir dringend verpflichtend für alle kommerziellen europäischen Flugzeuge, inklusive der Regional- und Billigfluglinien, einen AED.

Um auf Nachfrage geeignete notfallmedizinische Hilfestellung geben zu können, soll medizinisches Personal wissen, welches medizinische Equipment an Bord existiert und welche Situation sie vorfinden werden [505]. Die Verbreitung unterstützender Informationen (wie z. B. das "Doktor-an-Bord-Programm“, welches 2006 durch die Lufthansa und Austrian Airlines eingeführt wurde) soll für mitreisende Ärzte gefördert werden.

\section{Kreislaufstillstand in Rettungshubschraubern und Ambulanzflugzeugen}

\section{Einführung}

Luftrettungsdienste arbeiten entweder mit einem Rettungshubschrauber („helicopter emergency medical service“, HEMS) oder mit einem Ambulanzflugzeug, das üblicherweise kritisch kranke Patienten direkt zu spezialisierten Zentren bringt und einen Sekundärtransfer zwischen Krankenhäusern anbietet. Ein Kreislaufstillstand während des Fluges kann bei beiden, sowohl bei Patienten, die von einem Unfallort abtransportiert, als auch bei kritisch kranken Patienten, die von einem Krankenhaus zum anderen gebracht werden, vorkommen [521, 522]. In einer retrospektiven Analyse von 12.140 medizinischen Lufttransporten war die Inzidenz, einen Kreislaufstillstand zu erleiden, niedrig $(1,1 \%)$. $34 \%$ waren medizinische Patienten und $57 \%$ Patienten mit Trauma. In der medizinischen Gruppe war die ROSCRate $75 \%$ [523].

Der Umfang der möglichen Behandlung an Bord eines Ambulanzflugzeugs variiert und hängt von medizinischen und technischen Faktoren, wie z. B. Kompetenzen und Zusammensetzung der Crew, Kabinengröße und Equipment, ab. Idealerweise sind alle medizinischen Maßnahmen vor dem Flug abgeschlossen, sodass ein Bedarf an 
ungeplanten Behandlungen während des Fluges, vermieden werden kann.

\section{Flugvorbereitungen}

Bei der Vorbereitung zum Transport eines kritisch kranken Patienten muss sichergestellt sein, dass jedes notwenige Monitoring angebracht und betriebsbereit ist, dass der i.v.-Zugang gesichert und gut zugänglich ist und dass alle Medikamente und die Ausrüstung während des Fluges verfügbar sind.

\section{Diagnostik}

Bei Monitor überwachten Patienten können eine Asystolie und die schockbaren Rhythmen (VF/pVT) schnell erkannt werden. Jedoch eine PEA zu erkennen kann speziell unter Sedierung oder Allgemeinanästhesie schwierig sein. Ein unerwarteter Bewusstseinsverlust (bei gefährdeten Patienten), Veränderungen in der EKG-Ableitung und der Verlust des Pulsoximetersignals, sollen einen Patienten- und Pulscheck auslösen. Ein plötzlicher Abfall des endtitalen $\mathrm{CO}_{2}$ Werts bei beatmeten Patienten oder der Verlust der Kurve bei Spontanatmenden mit endtitaler $\mathrm{CO}_{2}$-Messung sind ebenfalls Hinweise auf einen Kreislaufstillstand.

\section{Behandlung}

Kreislaufstillstände in Luftrettungsdiensten sollen gemäß dem universellen ALS-Algorithmus behandelt werden. Beginnen Sie mit Thoraxkompressionen und Beatmungen unmittelbar nach Feststellen des Kreislaufstillstands, bringen Sie das Monitoring, falls noch nicht geschehen, an, und gehen Sie nach dem universellen ALS-Algorithmus vor [168]. Wenn bei einem Monitor überwachten Patienten ein schockbarer Rhythmus $(\mathrm{VF} / \mathrm{pVT})$ erkannt wird und die Defibrillation schnell durchgeführt werden kann, wird unmittelbar eine Serie von bis zu drei aufeinanderfolgenden Defibrillationen vor dem Beginn der Thoraxkompressionen abgegeben. In einer USStudie gelang nach der Defibrillation bei $33 \%$ der Patienten ein ROSC, ohne dass sie Thoraxkompressionen erhalten hatten [523].

Um einen besseren Zugang zum Patienten zu haben, kann in kleineren
Hubschraubern mit nicht ausreichendem Platz für eine effektive Wiederbelebung eine Notfalllandung notwendig werden.

Da mechanische Reanimationshilfen Thoraxkompressionen mit hoher Qualität ermöglichen, soll deren Einsatz aufgrund des eingeschränkten Platzangebots in einem Luftrettungsflugzeug erwogen werden [248, 524]. Wenn ein Kreislaufstillstand während des Fluges als wahrscheinlich erachtet wird, muss die mechanische Reanimationshilfe vorbereitet werden [50, 525].

\section{Kreislaufstillstand während Sportveranstaltungen}

\section{Reanimation auf dem Spielfeld}

\section{Einführung}

Der plötzliche und unerwartete Kollaps eines Sportlers auf dem Spielfeld, der nicht mit Körperkontakt oder einem Trauma assoziiert ist, hat möglicherweise eine kardiale Ursache und braucht, um dem Betroffenen ein Überleben zu ermöglichen, schnelles Erkennen und eine effektive Behandlung. Der plötzliche Herztod ist die häufigste Todesursache von Sportlern während des Wettkampfs oder Trainings. Angaben über die Inzidenz variieren in Abhängigkeit mit der Messmethode. Kürzlich wurde die Inzidenz mit 1:11.394 bei Basketballspielern, 1:21.293 bei Schwimmern und 1:41.695 bei Sportlern im freien Gelände angegeben (Inzidenz als Ausdruck der Anzahl der Athleten pro Jahr, [526]). Bei unter 35-Jährigen sind hypertrophe Kardiomyopathie (HCM) und arrhythmogene rechtsventrikuläre Kardiomyopathie (ARVC) die häufigsten Ursachen, während die arteriosklerotische koronare Herzkrankheit für $80 \%$ der plötzlichen Herztode der über 35-Jährigen verantwortlich ist [527]. Angeborene Koronararterienabnormalitäten wurden bei $12-33 \%$ der Sportler beschrieben [528].

Commotio cordis, die Unterbrechung des Herzrhythmus durch einen Schlag auf das Präkordium, hat eine Inzidenz von $3 \%$ [529]. Der Schlag muss den Thorax in der Silhouette des Herzens in einem Zeitfenster von 20 ms während des Anstiegs der T-Welle treffen [530]. Die in den letzten Jahren berichtete gesamte Überlebensrate einer Commotio cordis hat sich auf bis zu $58 \%$ verbessert [531].

Was auch immer die Ursache für den plötzlichen Kollaps eines Sportlers ist, er soll eine unmittelbare Reaktion der Offiziellen oder des medizinischen Teams auslösen. Die Standardreanimationsmaßnahmen müssen mit den unten aufgeführten zusätzlichen Überlegungen angewendet werden.

\section{Zugang}

Dem medizinischen Team soll unverzüglich Zugang zum Spielfeld gewährt werden. Es ist wichtig, dass es die Zugangsregeln zum Spielfeld beachtet, und es bleibt zu hoffen, dass die Offiziellen auf dem Spielfeld das Kollabieren des Sportlers erkennen, auf ihn aufmerksam werden und das Spiel unterbrechen, sodass für die Helfer der sichere Zugang zum Wettkämpfer gewährleistet ist.

Wenn es bei einem informellen Wettkampf oder beim Training kein medizinisches Team gibt, liegt es in der Verantwortung des Schiedsrichters, des Trainers oder der anderen Sportlerkollegen, den Kollaps des Sportlers zu erkennen und den Hilferuf sowie die Reanimation zu veranlassen.

\section{Hilferuf}

Um dem kollabierten Sportler die bestmögliche Überlebenschance zu geben, ist der Hilferuf essenziell. Es ist entscheidend, dass Sportoffizielle, Trainer und Sportorganisatoren einen Plan zum Vorgehen bei einem medizinischen Kollaps oder Trauma haben. Damit der Ambulanz der bestmögliche Zugang zum Notfallort ermöglicht wird, kann dieser Plan in der einfachsten Form - Sicherstellung der Verfügbarkeit eines Mobiltelefons und Kenntnis der Postadresse des Sportareals (Spielfeld oder Vereinsheim) - verfasst sein. Es bleibt zu hoffen, dass mehr Offizielle und Trainer in BLS und der Anwendung eines AED ausgebildet werden.

\section{Reanimation}

Wenn der Sportler bewusstlos ist und nicht normal atmet, beginnen Sie mit $B L S$. Ist ein $A E D$ verfügbar, bringen Sie diesen an, und folgen Sie den An- 
weisungen; wenn der AED die Abgabe eines Schocks empfiehlt, könnte der zugrunde liegende Rhythmus ein Kammerflimmern sein und auf die Defibrillation reagieren.

Das Spielfeld ist oft eine offene Arena und kann bei einem größeren Wettkampf durch Tausende lokale Zuschauer und Fernsehzuschauer unter Beobachtung stehen. Obwohl die Behandlung nicht verzögert werden darf, kann der Transport in eine ruhigere und privatere Umgebung für das Fortsetzen der Behandlung erwogen werden. Wenn es keine unmittelbare Reaktion auf die Behandlung, aber ein organisiertes medizinisches Team gibt, kann dieser Transport nach der Abgabe von drei Defibrillationsversuchen erfolgen, aufgrund der Überlegung, dass die ersten drei Schocks die höchste Effektivität bei einer Defibrillation haben. Mit einem Transport sollen alle einverstanden sein, und er muss in Schritten vollzogen werden, die kontinuierliche Thoraxkompressionen ermöglichen. Gibt es kein medizinisches Team oder ist ein Defibrillator nicht sofort verfügbar, muss BLS solange fortgesetzt werden, bis weitergehende Versorgung eintrifft.

Wenn der Sportler auf die Wiederbelebung anspricht, muss er umgehend für weitere Untersuchungen und Behandlungen in das nächstgelegene Kardiozentrum verlegt werden. Aufgrund der Gefahr, dass der Rhythmus wieder umschlagen kann, soll der Transport durch den Rettungsdienst erfolgen.

\section{Vorbeugung}

Im Bestreben, den plötzlichen Herztod vorherzusehen und zu verhindern, haben die Medizinische Kommission des Internationalen Olympischen Kommitees (IOC Medical Commission 2014) und viele andere Sportföderationen ein kardiologisches Screening für alle Sportler empfohlen. Dennoch gibt es eine anhaltende Debatte über die anzuwendende Methode und die Population, die untersucht werden soll [532].

\section{Wasserrettung und \\ Ertrinkungsunfall}

\section{Einführung}

Ertrinken ist ein häufiger Grund für einen Unfalltod [533]. Sofortige und effektive Hilfe von Laien, ausgebildeten Helfern und medizinischem Personal kann zwischen Leben und Tod entscheiden [534-536]. Diese Leitlinien zeigen die Empfehlung zur initialen Rettung und Reanimation von Ertrinkungsopfern. Sie sind für professionelle Mitarbeiter im Gesundheitswesen und verschiedene Gruppen von Laienhelfern (z. B. Rettungsschwimmer, Rettungsbootbesatzungen, Bademeister und Wasserrettungsteams), die eine spezielle $\mathrm{Zu}$ ständigkeit in der Versorgung von Ertrinkungsopfern haben, ausgelegt.

\section{Epidemiologie}

Die Weltgesundheitsorganisation (WHO) berichtet, dass in jeder Stunde eines jeden Tages mehr als 40 Menschen ihr Leben durch Ertrinken verlieren; über 372.000 Todesfälle jedes Jahr [537]. Die WHO weist darauf hin, dass die wahre Zahl an Ertrinkungsopfern weltweit noch viel höher ist. Mehr als $90 \%$ dieser Todesfälle ereignen sich in Niedrig- und Mittellohnländern. Die Inzidenz für Ertrinkungsfälle variiert zwischen den Ländern, dabei ist sie im Osten Europas am häufigsten [533]. Die Risikogruppen variieren ebenfalls zwischen den einzelnen Ländern, i. Allg. sind Männer häufiger betroffen als Frauen. Die meisten unbeabsichtigten Ertrinkungsfälle treffen Kinder, die nicht schwimmen können. In Ländern in denen Freizeit am Wasser unter Alkohol- und Drogeneinfluss üblich sind, stellen junge Erwachsene eine zweite Risikogruppe dar [538, 539]. Meist verbunden mit einem Unfall und körperlichen Aktivitäten in Wassernähe, berichten manche Länder auch über die Zunahme an Ertrinkungsfällen in der Altersgruppe der über 70-Jährigen. Ertrinkungsunfälle sind am häufigsten in Binnengewässern (z. B. Seen, Flüsse) und während der Sommermonate [538-540].

\section{Definitionen, Klassifikationen und Berichtswesen}

Das „International Liaison Committee on Resuscitation“ (ILCOR) definiert den Ertrinkungsunfall als Prozess der aus einer primären respiratorischen Beeinträchtigung durch Eintauchen und/oder Untertauchen in ein flüssiges Medium resultiert. Diese Definition impliziert, dass eine Flüssigkeits-Luft-Grenzfläche am Beginn des Atemwegs des Betroffenen vorhanden sein muss, die verhindert, dass das Opfer Luft atmen kann. Das Opfer kann nach diesem Prozess leben oder sterben, es war per Definition auf jeden Fall in einen Ertrinkungsunfall verwickelt [541]. Als Submersion wird bezeichnet, wenn das Gesicht unter Wasser oder mit Wasser bedeckt ist. Der Atem- und Kreislaufstillstand tritt innerhalb einiger Minuten nach Submersion ein. Als Immersion wird dagegen bezeichnet, wenn der Kopf über Wasser bleibt. Meistens ist das durch das Tragen einer Rettungsweste der Fall. Bei einer Immersion bleibt das Opfer mit einem offenen Atemweg untergetaucht und erleidet eine Hypothermie. Es kann zur Aspiration von Wasser kommen, wenn dieses über das Gesicht des Opfers spitzt oder wenn der Betroffene mit dem Gesicht im Wasser bewusstlos wird. Den Unterschied zwischen Submersion und Immersion zu erkennen ist für das Verständnis der Epidemiologie, Pathophysiologie, des klinischen Verlaufs und prognostischer Parameter zwischen den beiden Ertrinkungsarten wichtig.

Wenn der Verunfallte gerettet ist, ist der Prozess des Ertrinkungsunfalls unterbrochen, man spricht dann von einem nicht tödlichen Ertrinkungsunfall. Wenn die Person zu irgendeinem Zeitpunkt als Folge des Ertrinkungsunfalls verstirbt, spricht man von einem tödlichen Ertrinkungsunfall. Vermeiden Sie Begriffe wie: trockenes und nasses Ertrinken, aktives und passives Ertrinken, stilles, sekundäres Ertrinken und Beinahe-Ertrinken [541]. Um die Übereinstimmung der Informationen zwischen Studien zu verbessern, nutzen Sie die Utstein-StyleDokumentationsvorlage für Ertrinkungsunfälle, um über das Outcome von Ertrinkungsereignissen zu berichten [542]. 


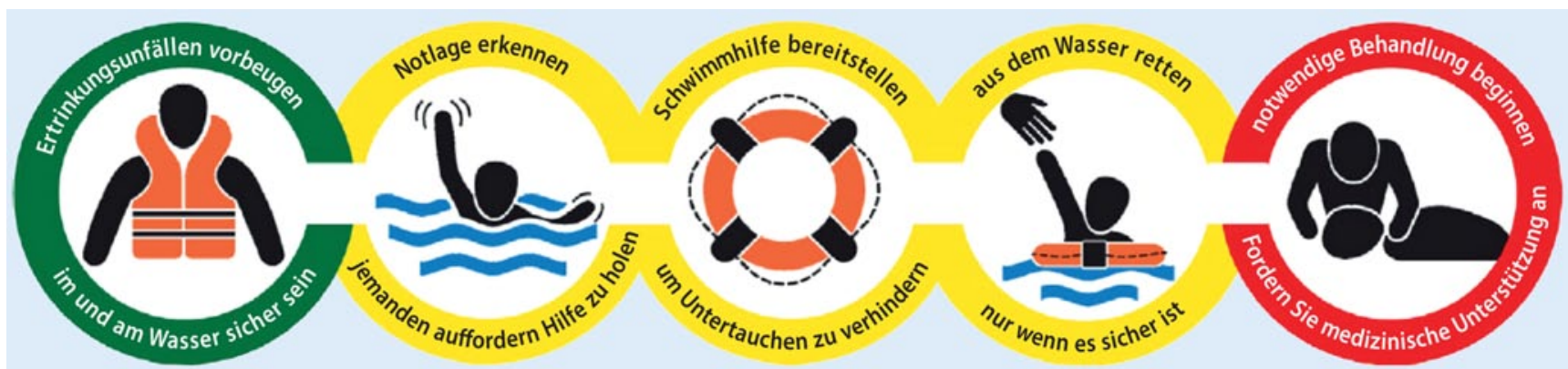

Abb. 5 \ Überlebenskette beim Ertrinkungsunfall [535]. (Reproduziert mit freundl. Erlaubnis von Elsevier Ireland Ltd)

\section{Pathophysiologie}

Es wurden detaillierte Zusammenfassungen über die Pathophysiologie des Ertrinkungsunfalls publiziert [536, 541, 543, 544]. Kurz zusammengefasst wird das Opfer infolge einer Submersion initial aufgrund eines Reflexes den Atem anhalten. Während dieser Zeit schluckt der Betroffene häufig Wasser. Wenn der Atem weiter angehalten wird, entwickelt sich eine Hypoxie und Hyperkapnie. Ein reflektorischer Laryngospasmus kann temporär das Eindringen von Wasser in die Lungen verhindern. Eventuell können diese Reflexe nachlassen, und das Opfer aspiriert Wasser. Das interessante Kernmerkmal in der Pathophysiologie des Ertrinkungsunfalls ist, dass es, bevor der Kreislaufstillstand eintritt, als Konsequenz der Hypoxie zur Bradykardie kommt. Bei der Reanimation ist die Korrektur der Hypoxämie durch Beatmung entscheidend, sie kann in manchen Fällen als alleinige Maßnahme zum Wiedereinsetzten des Kreislaufs (ROSC) führen; vielleicht auch deshalb, weil ein vorhandener Kreislauf gar nicht erkannt wurde [545-549].

\section{Überlebenskette des Ertrinkungsunfalls}

Die Überlebenskette beim Ertrinkungsunfall beschreibt fünf wichtige Glieder, die die Überlebenschancen nach einem Ertrinkungsunfall verbessern (• Abb. 5; [535]). Die ersten beiden Kettenglieder beziehen sich auf die Vorbeugung und die Erkennung der Notlage [550, 551]. Dieses Kapitel zeigt Leitlinien zur Rettung aus dem Wasser, zur initialen Reanimationsphase und zur Phase der Nachsorge nach erfolgreicher Reanimation.

\section{Wasserrettung}

\section{Laienhilfe}

Laien spielen eine wichtige Rolle bei den ersten Schritten der Rettung und Reanimation [534, 548, 552-555]. Gleichzeitig gibt es aber auch Laien, die bei einem Rettungsversuch selbst ums Leben gekommen sind, insbesondere dann, wenn der Ertrinkungsunfall in der Brandung oder in schnell fließendem Wasser stattfand [556]. Wenn immer möglich, sollen Laien versuchen, das Ertrinkungsopfer zu retten, ohne selbst ins Wasser zu gehen. Mit dem Betroffenen sprechen, ihn mit einem Rettungshilfsmittel (z. B. Stock oder Kleidern) versuchen zu erreichen (ein Seil oder ein schwimmfähiges Rettungshilfsmittel zu werfen, kann effektiv sein, wenn das Opfer nahe am Ufer ist). Wenn es notwendig ist, ins Wasser zu gehen (nutzen Sie ein schwimmfähiges Rettungshilfsmittel, einen Rettungsschwimmkörper oder ein Boot [535]). Es ist sicherer, zu zweit ins Wasser zu gehen als allein. Wenn Sie einen Rettungsversuch unternehmen, springen Sie niemals mit dem Kopf voraus ins Wasser. Sie können dabei den Sichtkontakt zum Opfer verlieren und laufen Gefahr, sich eine Wirbelsäulenverletzung zuzuziehen.

\section{Hilfe von Rettern mit Ausbildung}

Ausgebildete Retter sind oft Profis, die in Teams mit spezieller Ausrüstung die Suche und Rettung unterstützen. Wenn die Aktion länger dauert, versuchen die Teams, aus der möglichen Überlebenswahrscheinlichkeit eine Strategie zum weiteren Rettungsvorgehen abzuleiten. Aus diesem Grund hat die ILCOR spezifische prognostische Faktoren geprüft und festgestellt, dass eine Submersion von $<10$ min Dauer mit einer sehr hohen Chance für ein günstiges Outcome verbunden ist; eine Submersion, die > $25 \mathrm{~min}$ dauert, ist hingegen mit einer geringen Chance dafür verbunden [557]. Fragen nach Alter, Reaktionszeit des Rettungsdienstes, Salz- oder Süßwasser, Wassertemperatur und ob der Ertrinkungsunfall beobachtet war, sind nicht geeignet, um ein Überleben vorhersagen zu können. Submersion in eiskaltem Wasser kann das Zeitfenster für das Überleben verlängern und eine ausgedehntere Suchund Rettungsaktion rechtfertigen [558560].

\section{Reanimation im Wasser}

Ausgebildete Personen können eine Beatmung im Wasser durchführen, idealerweise mit der Unterstützung einer schwimmfähigen Rettungshilfe [545, 561, 562]. Wenn ein Retter, in der Regel ein Rettungsschwimmer, ein lebloses Ertrinkungsopfer in tiefem offenem Gewässer findet, kann er (wenn er darin ausgebildet ist) im Wasser mit Beatmungen beginnen, bevor er das Opfer ans trockene Ufer oder auf das Rettungsboot bringt. Manche Ertrinkungsopfer reagieren darauf. Wenn nicht, soll der Retter in Abhängigkeit von der lokalen Situation wie Seegang, Abstand zum Ufer, Verfügbarkeit eines Rettungsboots oder Helikopters entscheiden, ob er das Opfer so schnell wie möglich an Land bringt, also ohne weitere Beatmungen während des Rettungsschwimmens, oder ob er direkt im Wasser weiterbeatmet, bis die Mannschaft des Rettungsboots oder Helikopters eintrifft, um die Reanimation zu übernehmen. Eine Studie deutet darauf hin, dass die zweite Option mit einer höheren Überlebensrate verbunden ist [545]. 


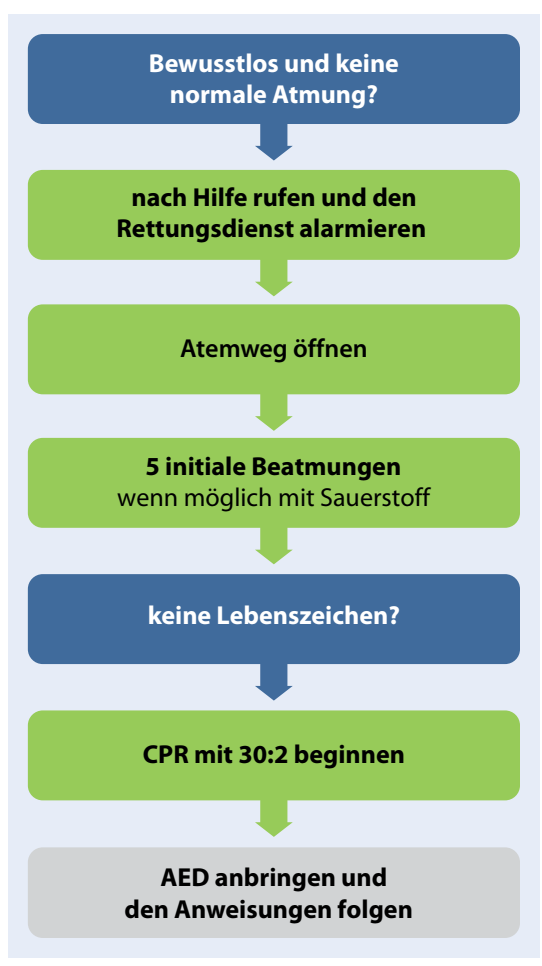

Abb. $6 \Delta$ Behandlungsalgorithmus Ertrunkener für Ersthelfer im Dienst

\section{Verbringen aus dem Wasser}

Holen Sie das Opfer sofort aus dem Wasser. Die Wahrscheinlichkeit, dass ein Ertrinkungsopfer eine spinale Verletzung erlitten hat, ist sehr gering [563]. Solange es keine Anamnese eines Sprungs in seichtes Wasser oder Zeichen einer schweren Verletzung nach Rutschen auf der Wasserrutsche, Wasserski, Kite-Surfing oder Bootsrennen gibt, sind spinale Vorsichtsmaßnahmen unnötig. Wenn das Opfer pulslos ist und eine Apnoe hat, holen Sie es so schnell wie möglich aus dem Wasser. Achten Sie darauf, Flexion und Extension des Nackens möglichst gering zu halten. Eine Hypovolämie nach längerer Immersion kann bei der Rettung einen Kollaps oder Kreislaufstillstand verursachen. Belassen Sie das Opfer während und nach der Rettung aus dem Wasser in einer horizontalen Position.

\section{Die initiale Reanimation nach Rettung aus dem Wasser}

Wenden Sie die Standard-BLSMaßnahmen an, indem Sie die Reaktion auf Ansprache prüfen, den Atemweg öffnen und nach Lebenszeichen suchen. Ein Ertrinkungsopfer, das nach wenigen Minuten Submersion aus dem Wasser gerettet wird, wird oft eine abnormale (agonale) Atmung zeigen. Verwechseln Sie das nicht mit einer normalen Atmung.

\section{Notfallbeatmung}

Der BLS-Ablauf bei Ertrinkungsopfern spiegelt die Wichtigkeit der schnellen Behandlung der Hypoxie (• Abb. 6). Die Insufflation soll eine Sekunde betragen und ist suffizient, wenn sich der Brustkorb hebt. Da die Lunge eine reduzierte Compliance und einen hohen Atemwegswiderstand hat, dauert die Insufflation manchmal länger als unter normalen Umständen. Der höhere Beatmungsdruck kann die Füllung des Magens mit Luft begünstigen, was zur Regurgitation und auch zur Verminderung des Herzzeitvolumens führen kann. Expertenmeinung legt nahe, dass der Druck auf das Krikoid durch ausgebildetes und geübtes Personal in Fällen ohne gesicherten Atemweg die Magenbeatmung reduzieren und die Beatmung bei Ertrinkungsopfern verbessern kann.

\section{Thoraxkompressionen}

Wenn das Ertrinkungsopfer nicht auf die initialen Beatmungen reagiert, soll es, bevor mit Thoraxkompressionen begonnen wird, auf einen stabilen Untergrund gelegt werden. Kompressionen im Wasser sind ineffektiv $[564,565]$. CPR wird im Verhältnis 30 Kompressionen zu 2 Beatmungen durchgeführt. Die meisten Ertrinkungsopfer haben einen sekundären Kreislaufstillstand durch Hypoxie. Bei diesen Patienten wird die CPR ohne Beatmungen ineffektiv sein und soll vermieden werden.

Sofern genügend Retter anwesend sind, soll der Helfer, die die Wasserrettung durchgeführt hat, nicht mehr an der Fortführung der CPR beteiligt werden, da er wahrscheinlich für eine qualitativ hochwertige Wiederbelebung zu erschöpft ist $[566,567]$.

\section{Automatisierte externe Defibrilla- tion}

Verschieben Sie den Einsatz des AED, bis die CPR begonnen hat. Trocknen Sie die Brust des Ertrinkungsopfers, bringen Sie die Defibrillatorpads an, und schalten Sie den AED ein. Defibrillieren Sie gemäß den Anweisungen des AED.

\section{Flüssigkeit in den Atemwegen}

In einigen Situationen können größere Mengen Schaum, der durch Vermischung von austretender Luft und Wasser entsteht, aus dem Mund des Patienten kommen. Verschwenden Sie keine Zeit, den Schaum zu entfernen, da er weiterhin austreten wird. Setzen Sie die Notfallbeatmung fort, bis ein ALS-Provider eintrifft, der das Opfer intubieren kann. Die Regurgitation von Mageninhalt und geschlucktem Wasser ist während der Reanimation von Ertrinkungsopfern häufig [568]. Wenn dies die Ventilation verhindert, soll das Opfer auf die Seite gedreht und das regurgitierte Material möglichst durch direktes Absaugen entfernt werden.

\section{Modifizierungen für den „Advanced Life Support"}

\section{Atemweg und Atmung}

Währender der Erstbeurteilung eines spontan atmenden Ertrinkungsopfers verabreichen Sie Sauerstoff im "high flow" (10-15 l/min), idealerweise mittels einer Sauerstoffmaske mit Reservoir [127]. Bei Opfern, die nicht auf diese Maßnahmen ansprechen, deren Bewusstseinslage eingeschränkt ist oder die einen Kreislaufstillstand haben, soll frühzeitig eine endotracheale Intubation mit kontrollierter Beatmung durch erfahrenes Personal in Erwägung gezogen werden. Eine schlecht pulmonale Compliance bedarf hoher Beatmungsdrücke und limitiert somit den Einsatz von supraglottischen Atemwegshilfen [569]. Vor der trachealen Intubation soll große Sorgfalt auf eine optimale Präoxygenierung angewendet werden. Die Flüssigkeit des Lungenödems verhindert vielleicht die Sicht auf den Larynx und macht eine kontinuierliche Absaugung nötig. Nach Verifizierung der Tubuslage soll die inspiratorische Sauerstoffkonzentration so gewählt werden, dass ein $\mathrm{SpO}_{2}$ (pulsoxymetrisch gemessene Sauerstoffsättigung) von 94-98\% erreicht wird [127]. Die Pulsoxymetrie kann bei Ertrinkungsopfern falsche Werte anzeigen [570]. Der PEEP („positive endexspiratory pressure") soll mindestens $5-10 \mathrm{~cm} \mathrm{H}_{2} \mathrm{O}$ betragen. Es können aber auch PEEP-Werte von 15-20 $\mathrm{cm} \mathrm{H}_{2} \mathrm{O}$ benötigt werden, wenn der Patient unter 
einer schweren Hypoxämie leidet [571]. Der Magen soll mit einer Magensonde entlastet werden.

\section{Kreislauf und Defibrillation}

Das Palpieren des Pulses zur Diagnostik eines Kreislaufstillstands als einziger Indikator ist nicht immer zuverlässig. Nutzen Sie so früh wie möglich Monitoring, wie das EKG, endtidales $\mathrm{CO}_{2}$ und Echokardiographie, um die Diagnose eines Kreislaufstillstands zu bestätigen.

Wenn das Opfer einen Kreislaufstillstand hat, verfahren Sie nach den ALSStandardprotokollen. Sollte das Opfer hypotherm sein, modifizieren Sie das Vorgehen nach den Empfehlungen zur Behandlung von Hypothermie (s. „Нypo-/ Hyperthermie“).

Nach längerer Zeit unter Wasser werden die meisten Opfer nach dem Wegfall des Wasserdruckes auf den Körper hypovoläm. Um die Hypovolämie auszugleichen, verabreichen Sie schnell Flüssigkeit i.v.. Dies soll präklinisch geschehen, wenn ein längerer Transport erwartet wird.

\section{Abbruch der \\ Reanimationsmaßnahmen}

Es ist bekanntermaßen schwierig, bei Ertrinkungsopfern die Entscheidung über den Abbruch von Reanimationsmaßnahmen zu treffen.

Kein einzelner Faktor kann mit Sicherheit ein gutes oder schlechtes Ergebnis vorhersagen.

Es passiert häufig, dass sich Entscheidungen, die in der Präklinik getroffen werden, später als inkorrekt herausstellen [572]. Führen Sie also die Reanimation so lange weiter, bis eindeutige Evidenz besteht, dass die Versuche zwecklos sind (z. B. schwere traumatische Verletzungen, Rigor Mortis, Fäulnis) oder eine zeitgerechte Einlieferung in ein medizinisches Versorgungszentrum nicht gewährleistet ist. Es wurde von mehreren Patienten mit gutem neurologischem Outcome berichtet, die $>25$ min unter Wasser waren. Dies sind seltene Fallberichte fast ausschließlich von Kindern in eiskaltem Wasser, bei denen eine Hypothermie vor der Hypoxie eingetreten ist oder von Patienten, die im Auto unter Wasser eingeschlossen waren $[558,559$,
573, 574]. Eine retrospektive Studie von 160 Kindern aus den Niederlanden fand ein extrem schlechtes Outcome, wenn ALS > 30 min brauchte, um einen ROSC wiederherzustellen [560].

\section{Reanimationsnachsorge}

\section{Salz- vs. Süßwasser}

Kleine Unterschiede in Elektrolytstörungen sind selten klinisch relevant und benötigen üblicherweise keine Therapie $[575,576]$.

\section{Lungenschädigung}

Der vorherrschende pathophysiologische Prozess in der Lunge wird durch Auswaschen und Funktionsstörung des Surfactants, alveolärem Kollaps, Atelektasen und intrapulmonalem Shunten verursacht. Der Schweregrad der Lungenschädigung reicht von einer leichten selbst limitierenden Erkrankung bis hin zu therapierefraktärer Hypoxämie. Viele Ertrinkungsopfer haben ein hohes Risiko, ein akutes Lungenschädigungssyndrom („acute respiratory distress syndrome“, ARDS) zu entwickeln [578, 579]. Die extrakorporale Membranoxygenierung (ECMO) wurde für Fälle von refraktärem Kreislaufstillstand, refraktärer Hypoxämie und in ausgewählten Fällen von Eiswasser-Ertrinken angewendet, jedoch mit niedrigen Erfolgsraten [580-583].

Pneumonien sind häufig nach Ertrinkungsunfällen. Eine prophylaktische Antibiotikagabe hat keinen Nutzen gezeigt, kann aber erwogen werden, wenn das Ertrinken in schwer kontaminiertem Wasser geschehen ist (z. B. Abwasser [584]). Verabreichen Sie ein Breitbandantibiotikum, wenn sich im weiteren Verlauf eine Infektion entwickelt [585-587].

\section{Neurologisches Outcome}

Das Neurologische Outcome, v. a. schwere permanente neurologische Schäden, ist primär durch die Dauer der Hypoxie bestimmt. Es wurde versucht, das neurologische Outcome durch den Einsatz von Barbituraten, Hirndruckmessung (ICP) und Steroiden positiv zu beeinflussen. Keine dieser Interventionen konnten eine Verbesserung des Outcomes erreichen [588].
Wildnis- und Umweltnotfälle

\section{Schwieriges Gelände und abgelegene Gegenden}

\section{Geographische und meteoro- logische Überlegungen}

Im Vergleich zu städtischen Gebieten gibt es Einsatzorte, die schwierig zu erreichen und weit von organisierter medizinischer Versorgung entfernt sind. Ungeschütztes und steiles Gelände kann zu anspruchsvoller und gefährlicher Rettung führen. Die Chance auf ein gutes Outcome nach Kreislaufstillstand kann durch verzögertes Erreichen und verlängerte Transportwege verschlechtert werden. Des Weiteren sind einige Gegenden unwegsamer als städtische Zonen (z. B. verursacht durch Kälte, Wind, Nässe, extreme Helligkeit durch Lichtreflektionen auf Eis und Schnee). Menschliche und materielle Ressourcen sind möglicherweise stark eingeschränkt [589, 590].

Verglichen mit dem Sauerstoffpartialdruck auf Meereshöhe $\left(\mathrm{pO}_{2}\right.$ um $21 \mathrm{kPa} / 159 \mathrm{mmHg}$ ), wird der $\mathrm{pO}_{2}$ in der Höhe (> 1500 m ü.M.), in großer Höhe (3500-5500 m) und in extremer Höhe (>5500 m) zunehmend sinken und die physischen Aktivitäten der Retter einschränken.

Es gibt eine physiologische Grenze der Akklimatisierung (z. B. kurzzeitige Hyperventilation und erhöhtes Herzzeitvolumen; langfristiger Anstieg des Hämoglobins). Die höchste permanente Siedlung liegt auf $5100 \mathrm{~m}\left(\mathrm{pO}_{2} \mathrm{um}\right.$ $11 \mathrm{kPa} / 84 \mathrm{mmHg}$ ). Oberhalb von $7500 \mathrm{~m}$ ist das Risiko für eine tödliche akute Höhenkrankheit sehr hoch.

Es gibt keine epidemiologischen Daten zu den Ursachen von Kreislaufstillständen in großer Höhe. Wie auch immer, es ist denkbar, dass es eine primär kardiale Hauptursache (60$70 \%$ ) für den plötzlichen Kreislaufstillstand gibt. Dennoch erscheinen Systeme für öffentlich erreichbare Defibrillatoren („public access defibrillator“, PAD) sinnvoll. Öffentliche Defibrillatoren sollen in beliebten Skigebieten, hochfrequentierten Hütten und Restaurants, bei Großveranstaltungen und an beliebten Fernzielen ohne medizinische Versorgung positioniert werden [591]. In Gegenden, 
in denen Ärzte häufiger zu Bergrettungseinsätzen gerufen werden, verläuft die Versorgung vor Ort im Allgemeinen gemäß den Leitlinien [592].

\section{Entscheidungsfindung}

Eine kontinuierliche Überwachung und Behandlung während des Transports ist schwierig, da der Patient in einem Bergesack aus der rauen Umgebung gerettet und später gut isoliert auf einer Trage transportiert wird. Unter Umständen wird die CPR in der Qualität eingeschränkt oder gar unmöglich sein (z. B. während des Tragens, des Abseilens oder der Windenrettung). In gefährlichem und schwierigem Gelände, in dem kontinuierliche CPR unmöglich ist, wurde für hypotherme Patienten eine verzögerte $\mathrm{CPR}$ vorgeschlagen [45]. Mechanische Reanimationshilfen können helfen, die Qualität der CPR während schwierigen Bergungen und verlängerten Transportwegen $\mathrm{zu}$ verbessern.

\section{Transport}

Effektive, sichere Immobilisierung und Schienung reduzieren die Mortalität und Morbidität [593].

Wenn möglich, soll der Patient mit einem Luftrettungsmittel transportiert werden [593, 594]. Die Organisation der Luftrettung („Helicopter Emergency Medical Services“, HEMS) beeinflusst das Outcome [595-597].

\section{Höhenkrankheit}

Durch die steigende Anzahl an Touristen, die sich in der Höhe aufhalten, steigt auch die Anzahl derer mit kardiovaskulären und metabolischen Risikofaktoren für einen Kreislaufstillstand. Der $\mathrm{pO}_{2}$ fällt mit zunehmender Höhe, und die Sauerstoffschuld führt möglicherweise zur Manifestation der akuten Höhenkrankheit.

Personen, die sich oberhalb von $3500 \mathrm{~m}$ aufhalten, haben ein Risiko, folgende Pathologien zu entwickeln:

- akute Höhenkrankheit („acute mountain sickness“, AMS) mit Kopfweh, Übelkeit, Müdigkeit und Abgeschlagenheit,

- Höhenlungenödem („high altitude pulmonary oedema“, HAPO) mit schwerer Dyspnoe und Zyanose,
- Höhenhirnödem („high altitude cerebral oedema", HACO) mit Schrankenstörung, Desorientiertheit und Verwirrung.

Die Risikofaktoren erhöhen sich durch einen schnellen Anstieg und eine frühere Höhenkrankheit. Wenn nicht sofort therapiert wird, können das HAPO und das HACO schnell voranschreiten und $\mathrm{zu}$ Bewusstlosigkeit, schwerer respiratorischer Einschränkung, Kreislaufinstabilität und Kreislaufstillstand führen. Das Wichtigste ist der sofortige Abstieg auf niedrigere Höhen, Sauerstoffgabe (2$6 \mathrm{l} / \mathrm{min}$, Ziel $>90 \% \mathrm{SpO}_{2}$ ), Behandlung in einer tragbaren Überdruckkammer, bei einem HACO die Gabe von Dexamethason, 4-8 mg, alle $8 \mathrm{~h}$, bei HAPO, Nifidepine, $30 \mathrm{mg}$, alle $12 \mathrm{~h}$.

Die Reanimation in der Höhe unterscheidet sich nicht von der StandardCPR. Bei dem niedrigeren $\mathrm{pO}_{2}$ ist die CPR für den Helfer erschöpfender als auf Meereshöhe, und die Anzahl der effektiven Thoraxkompressionen sinkt bereits innerhalb der ersten Minute [598600].

Wann immer möglich, sollen mechanische Reanimationshilfen zum Einsatz kommen.

Häufig sind keine Ärzte anwesend, um Pflegekräfte und Rettungssanitäter dahingehend anzuleiten, wann eine Reanimation zu beenden ist.

Eine Reanimation kann unterlassen oder beendet werden bei einem Patienten ohne Lebenszeichen, wenn

- ein inakzeptables Risiko für den Retter besteht,

- der Retter erschöpft ist,

- eine extreme Umgebung die Reanimation verhindert,

- einer der folgenden Umstände zutrifft:

- Enthauptung,

- Durchtrennung des Rumpfes,

- Verbrennung des ganzen Körpers,

- mehrfache Durchtrennung des Körpers,

- steif gefrorenes Opfer,

- Atemwegsverlegung nach Lawinenverschüttung und einer Verschüttungszeit $>60 \mathrm{~min}$ (s. Lawinenverschüttung).
Eine Reanimation kann ebenfalls beendet werden, wenn die folgenden Kriterien vollständig erfüllt sind:

- unbeobachteter Verlust der Vitalzeichen,

- kein ROSC nach 20 min Reanimation,

- durch den AED zu keiner Zeit ein Schock empfohlen oder ausschließlich Asystolie im EKG,

- keine Hypothermie oder andere reversible Ursachen, die eine verlängerte Reanimationsdauer rechtfertigen.

In Situationen, in denen kein Transport und keine Therapie behandelbarer Ursachen möglich ist, ist eine weitere Reanimation zwecklos und die CPR sollte eingestellt werden. Diese Empfehlung muss im lokalen und rechtlichen Kontext interpretiert werden.

\section{Lawinenverschüttung}

\section{Einführung}

In Europa und Nordamerika gibt es jährlich zusammen 150 Tote in Schneelawinen. Meistens sind sie sportassoziiert und betreffen Skifahrer, Snowboarder und Schneemobilfahrer. Die Todesursache ist meistens Asphyxie, manchmal mit Trauma und Hypothermie zusammen. Prognostische Faktoren sind: Schweregrad der Verletzungen, Dauer der kompletten Verschüttung, Verlegung der Atemwege, Kerntemperatur und das Serumkalium.

Komplett verschüttete Opfer sterben an Asphyxie innerhalb von $35 \mathrm{~min}$, wenn der Atemweg verlegt ist. Die durchschnittliche Auskühlgeschwindigkeit liegt im Bereich von $0,6-9^{\circ} \mathrm{C} / \mathrm{h}[603,604]$, im Mittel bei $3{ }^{\circ} \mathrm{C} / \mathrm{h}$ [602]. Moderate bis schwere Hypothermie wird nach $60 \mathrm{~min}$ Verschüttung wichtig, wenn der Atemweg frei ist. Das höchste dokumentierte und jemals überlebte Kalium eines Lawinenverschütteten ist $6,4 \mathrm{mmol} / \mathrm{l}[601,605-$ 607]. Die Überlebensrate von Lawinenopfern, die einen Kreislaufstillstand erlitten, liegt zwischen 7-17\% [605, 606]. Die Überlebensmuster unterscheiden sich zwischen den Ländern, abhängig vom $\mathrm{Ge}$ lände, dem Klima und der präklinischen medizinischen Versorgung [56, 608-610]. 


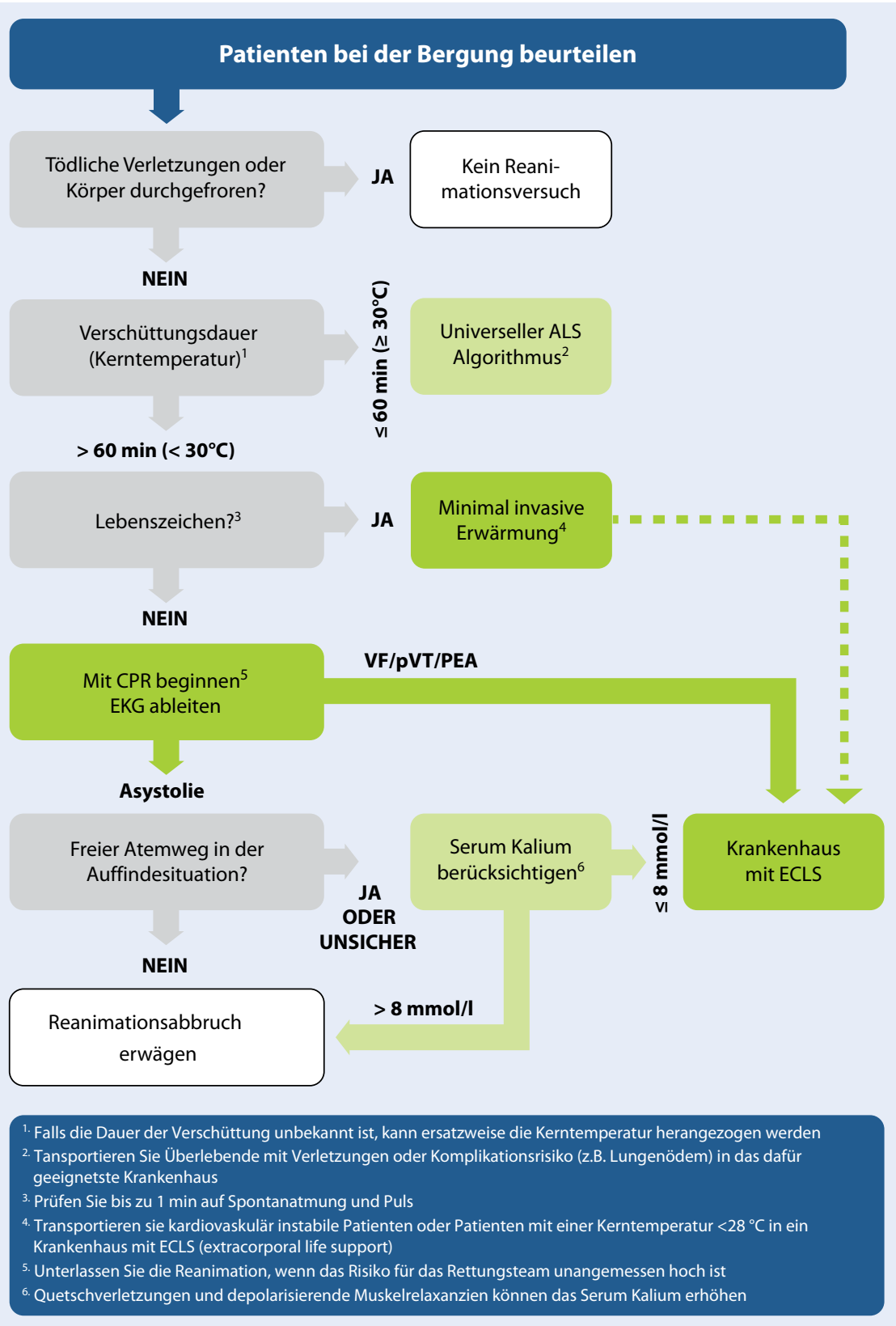

Abb. 7 \ Lawinenunfall Algorithmus. Managment des vollständig Verschütteten (ECLS, extracorporeal life support)

\section{Entscheidungsfindung vor Ort}

Es ist unwahrscheinlich, dass Lawinenopfer überleben, wenn sie:

- >60 min verschüttet waren (oder initiale Kerntemperatur $<30^{\circ} \mathrm{C}$ ) und einen Kreislaufstillstand mit verlegtem Atemweg bei der Bergung haben,

- verschüttet waren und im Kreislaufstillstand bei der Bergung ein initiales Kalium $>8 \mathrm{mmol} / \mathrm{l}$ haben.
Für alle anderen Lawinenopfer, die keine offensichtlichen letalen Verletzungen aufweisen, sind alle Wiederbelebungsmaßnahmen, einschließlich extrakorporaler Wiedererwärmung indiziert.

Lawinen entstehen in Gegenden, die für die Retter kurzzeitig schwer zu erreichen sind, und bei Verschüttungen sind häufig mehrere Opfer betroffen.

Die Entscheidung, alle Wiederbelebungsmaßnahmen zu beginnen, soll von der Anzahl der Opfer, den verfügbaren Ressourcen und von der Über- lebenswahrscheinlichkeit abhängig gemacht werden [601]. Da die aktuellen Leitlinien nicht gut eingehalten werden $[611,612]$. wird die Verwendung von standardisierten Checklisten empfohlen [613].

\section{Management von komplett ver- schütteten Lawinenopfern}

Der Algorithmus für die Behandlung von verschütteten Lawinenopfern ist in

- Abb. 7 dargestellt.

- In jedem Fall soll der Körper vorsichtig und unter Schutz der Wirbelsäule befreit werden.

- Erwägen Sie, die Wiederbelebung abzubrechen, wenn an der Einsatzstelle ein erhöhtes Risiko für das Rettungsteam besteht, das Opfer tödlich verletzt oder komplett gefroren ist.

- Bestimmen Sie die Dauer der Verschüttung. Wenn sie nicht zu ermitteln ist, kann die Kerntemperatur ersatzweise zur Entscheidungsfindung herangezogen werden.

- Wenn die Zeit der Verschüttung $\leq 60$ min beträgt (oder die initiale Kerntemperatur $\geq 30^{\circ} \mathrm{C}$ ) und ein Kreislaufstillstand gesichert ist, folgen Sie den Standard-ALS-Leitlinien (s. „Erweiterte lebensrettende Maßnahmen für Erwachsene“, [168]). Während der Wiederbelebung, sollen Sie die Kerntemperatur messen, das EKG beobachten, Sauerstoff applizieren und für Isolation sorgen. Legen Sie außerdem Wärmepackungen auf den Körperstamm. Flüssigkeit und Medikamente sollen nur dann verabreicht werden, wenn ein i.v.- oder i.o.-Zugang in wenigen Minuten zu etablieren ist. Die Wiederbelebungsmaßnahmen können bei einem normothermen asystolen Patienten beendet werden, wenn ALS nach 20 min nicht erfolgreich war und keine reversiblen Ursachen vorliegen (s. „Ethik und Endscheidungen am Lebensende“, [243]).

- Transportieren Sie Überlebende unter besonderer Beachtung von respiratorischen (z. B. Lungenödem) oder anderen systematischen Erkrankungen oder Verletzungen in die nächste geeignete Klinik. Führen 
Sie, wenn indiziert, eine spezifische traumatologische Versorgung durch. Das aufnehmende Krankenhaus muss die Möglichkeiten besitzen, aktive externe Erwärmung oder interne zentrale Wiedererwärmung durchzuführen.

- Wenn die Dauer der Verschüttung $>60$ min beträgt (oder die initiale Kerntemperatur $<30^{\circ} \mathrm{C}$ ) und der Kreislaufstillstand bestätigt ist, beginnen Sie mit der Wiederbelebung, und schließen Sie einen Monitor an. Findet sich irgendeine elektrische Aktivität oder beim asystolen Patienten ein offener Atemweg, führen Sie die Wiederbelebung weiter. Defibrillation, über den dritten Versuch hinaus, kann verzögert werden, bis eine Kerntemperatur $>30^{\circ} \mathrm{C}$ erreicht wird.

- Transportieren Sie alle Patienten, die kardiovaskulär instabil sind oder eine Kerntemperatur $<28^{\circ} \mathrm{C}$ besitzen, in ein ECLS Zentrum. Befolgen Sie, wenn nötig, die regionalen Hypothermieprotokolle.

- Wenn ein direkter Transport in ein Zentrum mit Herz-Lungen-Maschine innerhalb einer sinnvollen Frist z. B. luftgebunden nicht möglich ist, bestimmen Sie im nächstgelegenen Krankenhaus den Kaliumwert. Wenn der Kaliumwert $>8 \mathrm{mmol} / \mathrm{l}$ ist, erwägen Sie eine Beendigung der Wiederbelebung (nach Ausschluss von schweren Gewebeverletzungen und dem Einsatz von depolarisierenden Muskelrelaxantien).

\section{Blitzschlag und Verletzungen mit elektrischem Strom}

\section{Einführung}

Stromverletzungen sind relativ selten, aber mit potenziell verheerenden multisystemischen Folgen und einer hohen Morbidität und Mortalität verbunden, es gibt 0,54 Toten/100.000 Menschen/Jahr. Die meisten Stromunfälle geschehen in geschlossenen Räumen. Während bei Erwachsenen üblicherweise Hochvoltstromunfälle bei der Arbeit passieren, sind Kinder meist zu Hause von Niedervoltunfällen betroffen (220 V in Europa, Australien und Asien, $110 \mathrm{~V}$ in den USA und Kanada, [614]). Stromschläge durch Blitze sind selten, verursachen aber jährlich 1000 Tote [615].

Die Stromverletzungen entstehen durch den direkten Einfluss von Strom auf die Zellmembran und den glatten Gefäßmuskel. Die thermische Energie von Hochvoltstromschlägen verursacht ebenso Verbrennungen. Folgende Faktoren beeinflussen den Schweregrad der Verletzung: der Unterschied zwischen Gleich- und Wechselstrom, Spannung, Stromstärke, Widerstand, der Weg des Stromflusses und der Ort und die Dauer des Kontakts. Der Hautwiderstand wird durch Feuchtigkeit erniedrigt und erhöht das Risiko. Der Strom fließt den Weg des geringsten Widerstands; Gefäßnervenbündel in den Extremitäten sind Schädigungen besonders ausgesetzt. Kontakt mit Wechselstrom kann tetanische Kontraktionen des Skelettmuskels verursachen, was vielleicht das Loslassen der Stromquelle verhindert. Myokardiales oder respiratorisches Versagen führen direkt zum Tod.

- Respiratorisches Versagen kann durch eine Lähmung der zentralen Atemregulation oder der Atemmuskulatur verursacht werden.

- Fließt der Strom in der vulnerablen Phase (analog dem R-auf-TPhänomen) durch das Myokard, kann Kammerflimmern ausgelöst werden [616]. Elektrischer Strom kann durch Koronarspasmen auch Myokardischämien auslösen. Es gibt primäre Asystolien oder sekundäre durch Asphyxie nach Atemstillstand.

Strom, der durch das Myokard fließt, ist häufig tödlich. Transthorakaler (Handzu-Hand-)Stromfluss ist häufiger fatal als der vertikale (Hand-zu-Fuß) oder die Grätsche (Fuß-zu-Fuß).

Es können ausgedehnte Gewebeverletzungen entlang des Stromflusses entstehen. Zugehörige Verletzungen sind häufig. Durch die Druckwelle (hyperbar), durch Wegschleudern vom Ort des Kontakts wie auch durch tetanische Kontrakturen verursachte Folgen und Frakturen sind beschrieben worden.

\section{Blitzschlag}

Bei einem Blitzschlag fließen $>300 \mathrm{kV}$ innerhalb weniger Millisekunden. Der Großteil des Stroms eines Blitzes fließt über die Oberfläche des Körpers als sog. Außenüberschlag. Blitzschläge und industrieller Strom verursachen tiefe Verbrennungen am Ort des Kontakts. Die Eintrittsstellen des industriellen Stroms sind üblicherweise an den oberen Extremitäten, Händen und Handgelenken lokalisiert, während Kopf, Hals und Schultern von Blitzschlägen betroffen sind. Verletzungen können auch indirekt durch die Schrittspannung des Erdpotentials oder überspringenden Strom von Bäumen oder anderen Gegenständen, die vom Strom getroffen wurden, verursacht werden [617]. Explosion kann ein stumpfes Trauma verursachen [618]. Verletzungsmuster und Schwere eines Blitzschlags unterscheiden sich stark auch innerhalb der Individuen einer Gruppe [619-621]. Beim industriellen und häuslichen Stromschlag ist der Tod durch Herz- [620-624] Atem-Stillstand [617, 625] bedingt. Bei denjenigen, die den initialen Schock überleben, kann eine ausgedehnte Katecholaminausschüttung oder eine autonome Stimulation eintreten. Dies verursacht Hypertension, Tachykardie, unspezifische EKG-Veränderungen (einschließlich QT-Verlängerung und vorübergehende T-Inversionen) und Myokardnekrosen. Die Kreatininkinase wird aus Skelett und Herzmuskel freigesetzt. Blitze können zentrale und periphere Nervenschäden verursachen; Hirnblutungen und -ödeme und periphere Nervenschäden sind häufig. Die Mortalität durch Blitzschläge liegt bei $30 \%, 70 \%$ der Überlebenden haben eine bleibende signifikante Morbidität [626, 627].

\section{Diagnostik}

Die äußeren Umstände sind oft nicht bekannt. Einzigartige Muster von Hautläsionen wie Fiederung oder LichtenbergFigur sind ein typisches Symptom, das nur bei Patienten gesehen wird, die einen Blitzschlag erlitten haben [628]. Bewusstlose Opfer mit linearen oder punktförmigen Verbrennungen (Fiederung) sollen wie Blitzschlagopfer behandelt werden [617]. 


\section{Sicherheitsmaßnahmen}

Versichern Sie sich, dass die Stromquelle ausgeschaltet ist, und nähern Sie sich nicht dem Opfer, bevor das nicht sicher ist. Hochvoltstrom (höher als die Haushaltsspannung) kann durch Bögen oder durch den Boden in einem Umkreis von einigen Metern leiten. Es ist ungefährlich, sich Opfern nach einem Blitzschlag zu nähern und sie zu behandeln, auch wenn es sinnvoll wäre, in eine sicherere Umgebung zu wechseln, v. a. wenn Blitze innerhalb der letzten 30 min gesehen wurden [617].

\section{Wiederbelebung}

Vom Blitz getroffene Patienten versterben sehr wahrscheinlich, wenn sie im Kreislauf- oder Atemstillstand bleiben und nicht schnell behandelt werden. Sind mehrere Opfer gleichzeitig vom Blitz getroffen worden, sollen die Retter den Patienten mit Atem- oder Kreislaufstillstand die höchste Priorität einräumen. Patienten mit Atemstillstand benötigen nur eine Beatmung, um den hypoxisch bedingten Kreislaufstillstand zu vermeiden. Die Wiederbelebungsversuche von Blitzschlagopfern sind erfolgreicher als von Patienten mit anderen Ursachen für Kreislaufstillstände. Die Anstrengungen sind zielführend, auch wenn das Intervall bis zum Beginn der Maßnahmen verlängert sein kann. Weite oder nicht lichtreagible Pupillen sollen nicht als prognostisches Zeichen genutzt werden, v. a. wenn der Patient von einem Blitz getroffen wurde [617].

Beginnen Sie ohne Verzögerung mit den Basis- und den erweiterten lebensrettenden Maßnahmen.

- Das Sichern der Atemwege kann durch elektrische Verbrennungen im Gesicht und Halsbereich erschwert sein. Eine frühe Intubation ist indiziert, da sich ausgedehnte Weichteilödeme entwickeln und dadurch die Atemwege verlegt werden können. Kopf und Wirbelsäulentraumata treten häufig nach Stromschlägen auf. Immobilisieren Sie die Wirbelsäule, bis eine Untersuchung durchgeführt werden kann.

- Muskellähmungen können speziell nach Hochvoltunfällen mehrere Stunden anhalten [627]. Unter- stützung der Atmung ist oft notwendig.

- Kammerflimmern ist die häufigste Rhythmusstörung nach Hochvoltwechselstromschlägen, behandeln Sie diese mit sofortiger Defibrillation. Asystolie ist nach Hochvoltgleichstromunfällen häufiger, verwenden Sie die Standardprotokolle für diese und andere Arrhythmien.

- Entfernen Sie schmorende Kleidung und Schuhe, um weitere thermische Schädigungen zu vermeiden.

- Es bedarf einer großzügigen Volumentherapie, wenn eine ausgedehnte Gewebezerstörung vorliegt. Erhalten Sie eine gute Urinausscheidung aufrecht, um die Ausscheidung von Myoglobin, Kalium und anderen Produkten aus Gewebeschäden zu verstärken [624].

- Erwägen Sie bei schweren thermischen Verletzungen eine frühzeitige chirurgische Intervention.

- Halten Sie bei Verdacht auf Kopf- und Halswirbelsäulentrauma die Wirbelsäulenimmobilisation aufrecht [629, 630].

- Führen Sie eine Zweituntersuchung durch, um Verletzungen durch tetanische Muskelkontrakturen oder Wegschleudern des Patienten auszuschließen.

- Stromschläge können in der Tiefe schwere Weichteilschäden verursachen, bei gleichzeitig relativ kleinen Hautläsionen, weil der Strom den neurovaskulären Bahnen folgt. Achten Sie genau auf Zeichen eines Kompartmentsyndroms, die einer Faszienspaltung bedürfen.

- Auch wenn es selten auftritt, ziehen Sie viszerale Schäden des Abdomens in Betracht, die möglicherweise durch direkten elektrischen Schaden verursacht wurden.

Es gibt widersprüchliche Berichte über die Verletzlichkeit des Fötus durch elektrischen Schock. Das Spektrum reicht von vorübergehenden unangenehmen Wahrnehmungen der Mutter mit keinem Effekt auf den Fötus, bis hin zu Plazentaablösungen, fötalen Verbrennungen oder intrauterinem Tod entweder sofort oder einige Tage später. Verschieden Faktoren, wie die Menge des Stroms und die Dauer des Kontakts, werden als Einflussgröße für das Outcome angesehen [632].

\section{Weiterbehandlung und Prognose}

Die sofortige Reanimation junger Stromschlagopfer mit Kreislaufstillstand kann zu Langzeitüberleben führen. Erfolgreiche Wiederbelebungen wurden nach verlängerten Reanimationsmaßnahmen berichtet.

Alle Überlebenden eines Stromschlags sollen in einem Krankenhaus überwacht werden, wenn sie Atem- oder Kreislaufprobleme oder Folgendes hatten:

- Bewusstlosigkeit,

- Kreislaufstillstand,

- EKG-Veränderungen,

- Weichteilschäden und Verbrennungen.

Schwere Verbrennungen (thermisch oder elektrisch), Myokardnekrosen, das Ausmaß der Verletzung des zentralen Nervensystems und in zweiter Linie Multiorganversagen bestimmen die Morbidität und die Langzeitprognose. In einigen Fällen wurde sogar von Knochenmarksembolien berichtet [633]. Es gibt keine spezifische Therapie für Stromschäden, und die Behandlung ist symptomorientiert. Die Prävention ist der beste Weg, die Häufigkeit und Schwere elektrischer Unfälle zu verringern.

\section{Massenanfall an Verletzten}

\section{Einführung}

Massenanfälle an Verletzten (MANV) sind seltene Ereignisse; sie erfordern mehr medizinische Versorgung, als die normalen Ressourcen leisten können. Von jährlich 19,8 Mio. Rettungsdiensteinsätzen in den USA haben nur 0,3\% den MANV als Alarmierungsgrund. Die Inzidenz wirklicher Katastrophen ist deutlich niedriger [634]. Das Internationale Rote Kreuz berichtet jährlich von 90 Katastrophen in Europa und 650 weltweit [635]. Der MANV oder die Katastrophe kann durch eine Vielzahl chemischer, biologischer, radioaktiver oder nuklearer Unfälle verursacht werden. Die traumatischen Ereignisse (z. B. Verkehrsunfälle, Anschläge, Naturoder Industriekatastrophen) sind in den 
entwickelten Ländern die Hauptursache für einen MANV [636]. Die initiale Sichtung ermöglicht es, die unterschiedlichen Behandlungsprioritäten zu erkennen. Im Gegensatz zu den üblichen Umständen wird bei einem MANV keine Wiederbelebung begonnen. Es gilt, eine Verzögerung effektiv zu behandelnder rettbarer Opfer zu vermeiden. Diese schwierige Entscheidung hängt von den verfügbaren Ressourcen und der Anzahl der Opfer ab.

\section{Triage und Entscheidungs- findung vor Ort}

\section{Sicherheit}

- Sicherheit hat höchste Priorität. Die zuerst eintreffenden Teams müssen die aktuellen Gefahren einschätzen und sofort geeignete Hilfe anfordern. Mehrere bewusstlose Patienten sollen den Retter an einen chemisch, biologisch oder radioaktiven Notfall denken lassen. Unerwartete Gefahren, wie z. B. Kohlenmonoxid, Cyanide oder andere industrielle Gifte, sind an kriminellen Tatorten oder in mit toxischen Substanzen verseuchten Umgebungen zu befürchten. Während der Sarinanschläge in Japan entwickelten $10 \%$ der 1363 Rettungskräfte Vergiftungserscheinungen, meist von primären Opfern in schlecht belüfteten Ambulanzen [637].

- Setzen Sie situationsgerecht Schutzmaßnahmen ein, und beachten Sie die potenziellen Risiken, bevor Sie sich einem Opfer nähern. Seien Sie sich bewusst, dass Schutzanzüge Ihre Möglichkeiten, adäquate Hilfe in kontaminierten Umgebungen zu leisten, einschränken können. Simulationsstudien haben dadurch geringeren Erfolg des erweiterten Atemwegsmanagement, mehr Zeitbedarf für i.v. oder i.o Zugänge und Schwierigkeiten beim Medikamente Aufziehen gezeigt [638-640].

\section{Triage}

- Benutzen Sie ein Triagesystem, um die Behandlungsprioritäten festzulegen, z. B. START („simple triage and rapid transport“), Newport Beach
Fire Departement, CA USA [641] oder SALT („sort-assess-lifesavinginterventions-treat/transport" [642, 643]). Die präklinischen Teams sollen bei der Triage eine Übertriage vermeiden. Bei Ankunft im Krankenhaus ist eine Reevaluation (Retriage) notwendig. Das Personal soll in allen Stadien der Notfallversorgung mit dem verwendeten Triagesystem vertraut sein.

- Wenn nach dem START-Triagesystem gesichtet wird, werden alle gehfähigen Patienten gebeten, den Unfallort zu verlassen, und bei denen, die nicht gehen können, wird der Status der Atemwege evaluiert. Atmet das Opfer nicht, wird der Atemweg frei gemacht (Kopf überstrecken und Kinn anheben, Esmarch-Handgriff). Beobachten und fühlen Sie nicht länger als $10 \mathrm{~s}$. Wenn ein Patient daraufhin nicht anfängt zu atmen, wird er als tot triagiert. Sollte ein nicht ansprechbares Opfer normal atmen, bringen Sie es in die stabile Seitenlage und markieren es mit höchster Priorität zur Behandlung. Weitere Untersuchungen, wie z. B. Atemfrequenz, Rekapillarisierungszeit und Ähnliches, hängt von den einzelnen Triageprotokollen ab.

- Die Entscheidung, ein MANVTriagesystem einzusetzen und Wiederbelebungen initial Toten vorzuenthalten, liegt in der Verantwortung einer medizinischen Führungsperson, nämlich der mit der höchsten medizinischen Kompetenz vor Ort.

- Ungenaue Triage kann für Patienten mit überlebbaren Verletzungen möglicherweise fatale Folgen haben. Medizinisches Personal soll regelmäßig die Verwendung des Triageprotokolls in Simulationen und Echtzeitübungen trainieren [644]. Moderne Technologien, wie z. B. Videospiele zu Lernzwecken, können die Leistung nachhaltig verbessern im Vergleich zu traditionellen Lehrmethoden, wie Kartenplanübungen [645]. Diese Übungen fördern schnelles und korrektes Erkennen derer, die lebensrettende Maßnahmen benötigen, und ver- mindern das Risiko, unangemessene Behandlungen in sinnlosen Fällen durchzuführen.

- Um die Anzahl der vermeidbaren Todesfälle zu reduzieren, ziehen Sie in Betracht, ältere Patienten und Hochenergietraumen bei der Triage in eine höhere Risikogruppe einzuteilen. Nach einem Flugzeugabsturz in den Niederlanden wurden 9\% mit kleineren Verletzungen (niedrigste Priorität) und $17 \%$ der gehfähigen Opfer untertriagiert und litten an schweren Verletzungen [646]. In der Nationalen Traumadatenbank (NTDB) wurden Patienten aller Triagegruppen auf Ergebnis und Mortalität verglichen. Von 322.162 Patienten, die der niedrigsten Gruppe zugeordnet wurden, starben 2046 vor der Krankenhausentlassung. Das Alter war die Hauptursache für die Untertriagierung [641].

- Führen Sie folgende lebensrettende Maßnahmen bei Patienten mit höchster Behandlungspriorität durch, um einen Kreislaufstillstand zu verhindern: Kontrollieren Sie massive Blutungen, machen Sie den Atemweg mit einfachen Mitteln frei, führen Sie eine Thoraxdekompression bei Spannungspneumothorax durch, verabreichen Sie Antidote, und bedenken Sie die initialen Beatmungen bei einem Kind mit Atemstillstand [642].

- Benutzen Sie bei Kindern ein spezielles Triage-Band oder ein kindgerechtes MANV-Triagesystem (z. B. JumpSTART, Team Life Support, Inc., FL, USA, http://www.jumpsarttriage. com) oder das universelle SALTSystem [647]. Falls diese nicht verfügbar sind, verwenden Sie jegliches System für Erwachsene.

\section{Besondere Patienten}

Kreislaufstillstand bei Patienten mit Begleiterkrankungen

\section{Asthma}

\section{Einführung}

Es wird geschätzt, dass weltweit ca. 300 Mio. Menschen jeder Altersgruppe 
und ethnischer Herkunft an Asthma leiden [648]. Die Häufigkeit von Asthma weltweit reicht von 1-18\% der Bevölkerung mit hohen Prävalenzen in einigen europäischen Ländern (Großbritannien, Skandinavien und den Niederlanden) und Australien [648, 649]. In den letzten Jahren hat die Häufung von Asthmafällen und die damit verbundene Morbidität und Mortalität ein Plateau erreicht und ist jetzt in einigen Ländern sogar ein wenig gesunken, v. a. bei Kindern und Jugendlichen [650653]. Die WHO schätzt, dass 15 Mio. Behinderungs korrigierte Lebensjahre („disability-adjusted life years“, DALY) pro Jahr durch Asthma verloren gehen. Dies stellt $1 \%$ der weltweiten Krankheitslast dar. Man geht davon aus, dass jährlich weltweit ca. 250.000 Tote durch Asthma zu beklagen sind. Diese Zahlen scheinen nicht mit den Daten der Prävalenz übereinzustimmen [648]. Es bestehen bereits nationale und internationale Leitlinien zur Behandlung von Asthma [654]. Die dortige Empfehlung fokussiert auf die Behandlung von hochkritischem Asthma mit nachfolgendem Kreislaufstillstand.

Patienten mit Risiko für einen asthmabedingten Kreislaufstillstand

Das Risiko fast fataler Asthmaanfälle ist nicht mit dem Schweregrad des Asthmas verknüpft [655]. Patienten mit dem höchsten Risiko, sind die mit

- einer Vorgeschichte eines fast letalen Asthmaanfalls, der eine Intubation und maschinelle Beatmung erforderte [656],

- Krankenhausaufenthalt und Notfallversorgung wegen Asthma im vergangenen Jahr [657],

- keinem oder geringem Gebrauch inhalativer Kortikosteroide [658],

- zunehmendem Gebrauch und erhöhter Abhängigkeit von Beta 2 -Mimetika [659],

- Angststörungen und Depressionen und/oder niedriger Therapieeinsicht [660, 661],

- Nahrungsmittelallergien [622].

Eine vertrauliche nationale Umfrage des Jahres 2014 in Großbritannien ergab, dass die Mehrzahl der asthmabedingten Todesfälle vor der Krankenhauseinweisung auftraten [663]. Verglichen mit jungen Er- wachsenen haben die älteren häufiger fast letale asthmabedingte Ereignisse und ein durch Komorbiditäten bedingtes höheres Mortalitätsrisiko [664].

\section{Ursachen für den Kreislaufstillstand}

Der Kreislaufstillstand bei Personen mit Asthma ist häufig das letzte Ereignis nach einer Phase der Hypoxämie und kann manchmal plötzlich auftreten. Kreislaufstillstände bei Asthmapatienten werden mit folgenden Faktoren in $\mathrm{Zu}$ sammenhang gebracht:

- Schwere Bronchospasmen und Schleimobstruktion führen zu Asphyxie (diese Voraussetzungen sind für einen Großteil, der asthmabedingten Todesfälle verantwortlich).

- Hypoxiebedingte Herzrhythmusstörungen sind der häufigste Grund für asthmabedingte Rhythmusstörungen [665]. Arrhythmien können auch durch stimulierende Medikamente (z. B. betaadrenerge Agonisten, Aminophyllin) oder Elektrolytentgleisungen hervorgerufen werden.

- Dynamische Überblähungen, wie z. B. ein autologer positiver endexpiratorisch Druck (AutoPEEP), treten manchmal unter maschineller Beatmung bei Asthmapatienten auf. Auto-PEEP wird durch Luft verursacht, die in die Lunge einund nicht wieder austritt. Der Druck baut sich schrittweise auf, verringert den venösen Rückstrom und senkt den Blutdruck.

- Spannungspneumothorax (oft beidseitig).

\section{Diagnosen}

Ein häufiges klinisches Zeichen ist das Giemen, wobei dessen Schweregrad nicht mit dem Ausmaß der Atemwegsobstruktion einhergehen muss. So kann ein Fehlen des Giemens Zeichen für eine kritische Atemwegsobstruktion sein, wohingegen eine Zunahme des Giemens evtl. Ausdruck einer guten Antwort auf die bronchodilatative Therapie ist. Das $\mathrm{SpO}_{2}$ spiegelt die alveoläre Hypoventilation nicht gut wider, vor allem, wenn Sauerstoff appliziert wird. $\mathrm{Zu}$ Beginn kann die $\mathrm{SpO}_{2}$ sinken, da BetaAgonisten neben der Bronchodilatation auch eine Vasodilatation auslösen und somit einen vermehrten intrapulmonalen Shunt verursachen.

Weitere Ursachen für Giemen sind: Lungenödem, chronisch obstruierende Lungenerkrankung (COPD), Pneumonie, Anaphylaxie, Fremdkörper, Lungenembolie und subglottische Raumforderung [666].

Der Schweregrad eines Asthmaanfalls ist in der $\bullet$ Tab. 3 dargestellt.

\section{Prävention des Kreislaufstill- stands}

Um einer Verschlechterung entgegenzuwirken, braucht ein Patient mit schwerem Asthmaanfall eine sofortige aggressive medikamentöse Therapie. Untersuchen und behandeln Sie den Patienten nach dem ABCDE-Schema. Patienten mit einer $\mathrm{SpO}_{2}<92 \%$ oder Zeichen eines lebensbedrohlichen Asthmas sind durch Hyperkapnie gefährdet und brauchen eine arterielle Blutgasanalyse. Diese Patienten sollen von erfahrenen Medizinern in einem Intensivbereich behandelt werden. Die spezielle medikamentöse Therapie und die Behandlungsabläufe unterliegen der jeweiligen örtlichen Verfahrensweise.

\section{Sauerstoff}

Der Einsatz von Sauerstoff soll so gewählt werden, dass ein Ziel von $\mathrm{SpO}_{2}=94-98 \%$ erreicht wird [127]. Sauerstoffmasken mit einer hohen Flussrate sind manchmal nötig. Eine fehlende Pulsoxymetrie soll den Gebrauch von Sauerstoff nicht ausschließen.

\section{Vernebelte $\boldsymbol{\beta} 2$-Agonisten}

Um einen akuten Asthmaanfall zu behandeln, sind die inhalativen $\beta_{2}$ Agonisten die Medikamente der ersten Wahl und sollen so früh wie möglich verabreicht werden. Intravenöse $\beta_{2}$ Agonisten sollen bei den Patienten zum Einsatz kommen, bei denen die inhalative Therapie nicht verlässlich durchgeführt werden kann. Der Eckpfeiler der akuten Asthmatherapie weltweit stellt die Verneblung von $5 \mathrm{mg}$ Salbutamol dar. Diese Dosis kann so lange wie nötig alle 1520 min wiederholt werden. In schweren Fällen ist möglicherweise eine kontinuierliche Salbutamol-Vernebelung angezeigt. Es sollen Vernebelungseinheiten, die mit hohen Flussraten Sauerstoff (mindestens 


\begin{tabular}{|c|c|c|}
\hline Asthma & \multicolumn{2}{|l|}{ Charakteristika } \\
\hline Fast letal & \multicolumn{2}{|c|}{$\begin{array}{l}\text { Erhöhter } \mathrm{paCO}_{2} \text { und/oder maschinelle Beatmung nötig mit erhöhten Beatmungs- } \\
\text { drucken }\end{array}$} \\
\hline \multirow{9}{*}{$\begin{array}{l}\text { Lebens- } \\
\text { bedrohlich }\end{array}$} & \multicolumn{2}{|c|}{ Einer der folgenden Faktoren: } \\
\hline & Klinisch & Meßwerte \\
\hline & Verwirrtheit, Koma & PEF $<33 \%$ bester Wert oder zu erwartende \\
\hline & Erschöpfung & $\mathrm{SpO}_{2}<92 \%$ \\
\hline & Arrhythmie & $\mathrm{paO}_{2}<8 \mathrm{kPa}$ oder $60 \mathrm{mmHg}$ \\
\hline & Hypotension & Normaler $\mathrm{paCO}_{2}(4,6-6,0 \mathrm{kPa}$ oder $35-45 \mathrm{mmHg})$ \\
\hline & \multicolumn{2}{|l|}{ Zyanose } \\
\hline & \multicolumn{2}{|c|}{ Stille Lunge ${ }_{, \prime}$,silent chest" } \\
\hline & \multicolumn{2}{|c|}{ Schwache Atemanstrengung } \\
\hline \multirow[t]{5}{*}{ Akut schwer } & \multicolumn{2}{|c|}{ Einer der folgenden Faktoren } \\
\hline & \multicolumn{2}{|c|}{ PEF $33-50 \%$ bester Wert oder zu erwarten } \\
\hline & \multicolumn{2}{|c|}{ Atemfrequenz $>25 / \mathrm{min}$} \\
\hline & \multicolumn{2}{|l|}{ Puls $>110 / \mathrm{min}$} \\
\hline & \multicolumn{2}{|c|}{ Unfähigkeit, Sätze in einem Atemzug zu beenden } \\
\hline
\end{tabular}

6 l/min) betrieben werden können, verfügbar sein. Die mit schwerem Asthma verbundene Hypoventilation, kann die effektive Aufnahme vernebelter Medikamente verhindern. $\beta_{2}$-Agonisten können auch zeitweise mit einem Dosieraerosol mit Inhalationshilfe verabreicht werden, wenn ein Vernebler nicht schnell verfügbar ist $[667,668]$. Im akuten Asthmaanfall bringt das zusätzliche Vernebeln von Adrenalin zum $\beta_{2}$-Agonisten keinen zusätzlichen positiven Effekt [669].

\section{Vernebelte Anticholinergika}

Vernebelte Anticholinergika (Ipratropium, 0,5 mg, alle 4-6 h) können bei schwerem Asthma oder bei den Patienten, die nicht auf $\beta_{2-}$ -Agonisten reagieren, eine zusätzliche Bronchodilatation bewirken, [670, 671].

\section{Vernebeltes Magnesiumsulfat}

Auch wenn ein geringer Anhalt dafür spricht, dass Magnesiumsulfat bronchodilatatorische Wirkungen besitzt, konnte eine Analyse von 16 randomisierten oder pseudorandomisierten Studien bei Kindern oder Erwachsenen mit Ashmaanfall, die Magnesium allein oder in Kombination mit inhalierten $\beta_{2}$-Agonisten (mit oder ohne Ipratropium) erhielten, weder die Lungenfunktion verbessern noch die Einweisungsrate ins Krankenhaus senken [673]. Kleine Studien mit Erwachsenen mit schwer exazerbiertem Asthma zeigten mit zusätzlich inhaliertem Magnesium eine verbesserte Lungenfunktion. Es gibt derzeit zu wenig beweisende Daten, um zu einer abschließenden Empfehlung zu kommen. Für die Behandlung des akuten Asthmas wird der Einsatz von inhalativem Magnesiumsulfat nicht empfohlen.

\section{Intravenöses Magnesiumsulfat}

Studien, die den Einsatz von i.v.Magnesiumsulfat untersuchten, haben widersprüchliche Resultate ergeben [672, 674, 675]. Eine Übersichtsstudie beschäftigte sich mit 14 Studien (3 davon waren Multicenterstudien) mit insgesamt 2313 Patienten. Es wurden Erwachsene oder ältere Jugendliche untersucht, die wegen akuten Asthmas in der Notaufnahme behandelt wurden. Dabei zeigte sich, dass eine einmalige Infusion mit 1,2 oder 2,0 $\mathrm{g} \mathrm{MgSO}_{4}$ über einen Zeitraum von 15-30 min signifikant die stationäre Aufnahme verringerte und für eine bessere Lungenfunktion sorgte im Vergleich zu den Patienten, die ein Placebo erhielten [„Odds Ratio“ (OR) =0,75; $95 \%$-Konfidenzintervall $(\mathrm{KI})=0,60$ $0,92,[676]]$. In fast allen Studien hatten die Patienten zumindest Sauerstoff, kurzwirksame vernebelte $\beta_{2}$-Agonisten und i.v.-Kortikosteroide bekommen, bevor sie in der Notaufnahme eintrafen.

Es wurde kein Unterschied bei anderer Ergebnisse, wie Intensiveinweisungen und Länge des Krankenhausaufenthalts, beobachtet.
Geben Sie Patienten mit akutem schwerem Asthma (beste oder vorhergesagte $\mathrm{PEF}<50 \%$ ) eine Einzeldosis Magnesiumsulfat i.v., wenn sie initial keine adäquate Reaktion auf eine inhalative Bronchodilatatortherapie zeigen. Die am häufigsten berichteten Nebenwirkungen von i.v.-Magnesiumsulfat sind Hitzewallungen, Müdigkeit, Übelkeit, Kopfschmerzen und Hypotonie.

\section{Intravenöse Kortikosteroide}

Eine frühe Anwendung von systemischen Kortikosteroiden zur akuten Asthmatherapie in der Notaufnahme reduziert signifikant die Häufigkeit von Krankenhauseinweisungen, v. a. für jene Patienten, die keine Kortikosteroidtherapie erhielten [677].

Zwar gibt es keinen Unterschied bei den klinischen Effekten zwischen oraler und i.v.-Applikation von Kortikosteroiden, aber es ist dennoch der i.v.Weg zu bevorzugen, da Patienten mit beinahe tödlichem Asthma erbrechen oder ggf. nicht schlucken könnten [678].

\section{Intravenöse Bronchodilatatoren}

Es gibt einen Mangel an definitiven Beweisen für oder gegen den Einsatz von i.v.-Bronchodilatatoren. Untersuchungen haben in erster Linie spontanatmende Patienten mit mittlerer bis lebensbedrohlicher Exazerbation des Asthmas einbezogen; Evidenz bei beatmeten Patienten mit lebensbedrohlichem Asthma oder Kreislaufstillstand ist rar.

Die Verwendung von i.v.Bronchodilatatoren soll generell auf Patienten beschränkt werden, die nicht auf eine inhalative Therapie ansprechen oder bei denen eine Inhalationstherapie nicht möglich ist (z. B. bei Patienten unter Beutel-Masken-Beatmung). Ein Cochrane-Review von i.v. im Vergleich zu inhalativen $\beta_{2}$-Agonisten fand keine Beweise für deren Nutzen, aber einige Hinweise auf vermehrte Nebenwirkungen im Vergleich zur inhalativen Anwendung [679]. Salbutamol kann entweder als langsame i.v.-Injektion ( $250 \mu \mathrm{g}$ i.v. langsam) oder kontinuierliche Infusion von $3-20 \mu \mathrm{g} / \mathrm{min}$ gegeben werden. 


\section{Aminophyllin}

Ein Cochrane-Review über die intravenöse Aminophyllin-Applikation fand keine Beweise für den Nutzen, aber eine höhere Inzidenz von Nebenwirkungen (Tachykardie, Erbrechen) im Vergleich zur Standardversorgung [680, 681]. Ob Aminophyllin einen Platz als zusätzliche Therapieoption nach der Behandlung mit inhalativen Beta-Agonisten und systemischen Kortikosteroiden hat, bleibt ungewiss. Sollte nach Expertenkonsultation eine Entscheidung zur Aminophyllin-i.v.-Applikation getroffen worden sein, so beginnt man mit einer Startdosis von $5 \mathrm{mg} / \mathrm{kg}$ über 20 30 min (ausgenommen bei Erhaltungstherapie), gefolgt von einer Infusion von $500-700 \mu \mathrm{g} / \mathrm{kg} / \mathrm{h}$. Theophyllin-Serumkonzentrationen sollen $<20 \mu \mathrm{g} / \mathrm{ml}$ gehalten werden, um eine Toxizität zu vermeiden.

\section{Leukotrienrezeptorantagonisten}

Es gibt nur wenige Daten über die Verwendung von i.v.Leukotrienrezeptorantagonisten [682]. Begrenzte Evidenz deutet auf eine Verbesserung der Lungenfunktion und eine nicht-signifikante Tendenz zu reduzierter Krankenhauseinweisung hin, wenn der i.v.-Leukotrienrezeptorantagonist Montelukast als Rescue-Therapie bei Erwachsenen mit akutem Asthma verwendet wird $[683,684]$. Weitere Untersuchungen sind erforderlich, um den Nutzen der Leukotrienrezeptorantagonisten in dieser Hinsicht zu bestätigen.

\section{Intravenöse Flüssigkeit und Elektrolyte} Schweres oder beinahe tödliches Asthma ist mit Dehydratation und Hypovolämie verbunden, und dies führt zu einer weiteren Beeinträchtigung der Zirkulation in Patienten mit dynamischer Hyperinflation der Lunge. Bei Anzeichen von Hypovolämie oder Dehydratation sind Kristalloide i.v. zu geben. $\beta_{2}$-Agonisten und Steroide können eine Hypokaliämie auslösen, die überwacht und, falls erforderlich, durch Elektrolyte korrigiert werden soll.

\section{Heliox}

Heliox ist eine Mischung aus Helium und Sauerstoff (üblicherweise 80:20 bzw. 70:30). Eine Metaanalyse von vier klinischen Studien lieferte keine Evidenz für die Anwendung von Heliox in der Initialtherapie von Patienten mit akutem Asthma [685].

\section{Adrenalin intramuskulär}

Manchmal kann es schwierig sein, schweres lebensbedrohliches Asthma von Anaphylaxie zu unterscheiden. Patienten mit schweren asthmaähnlichen Symptomen, aber ohne vorbestehende Lungenerkrankungen (Asthma, COPD) sollen so behandelt werden, als würde eine Anaphylaxie vorliegen. Unter diesen Umständen ist die Applikation von Adrenalin 0,5 mg i.m. nach den Anaphylaxieempfehlungen angemessen (s. „Anaphylaxie“).

\section{Einweisung auf die Intensivstation}

Als Experte soll ein Intensivmediziner alle Patienten beurteilen, die nicht auf eine Initialbehandlung reagiert haben oder Anzeichen von lebensbedrohlichem Asthma entwickeln. Die Aufnahme auf einer Intensivstation nach asthmaassoziiertem Kreislaufstillstand ist mit deutlich schlechterem Outcome verbunden als für Patienten ohne Kreislaufstillstand im Verlauf [686].

Erwägen Sie eine schnelle Narkoseeinleitung („rapid sequence induction“) und Intubation, wenn der Patient trotz Optimierung der medikamentösen Therapie folgende Zeichen aufweist:

- zunehmende Bewusstseinstrübung oder Koma,

- anhaltende oder sich verstärkende Hypoxämie,

- Verschlechterung einer respiratorischen Azidose trotz Intensivtherapie,

- große Unruhe, Verwirrtheit und Abwehr der Sauerstoffmaske (klinische Anzeichen von Hypoxämie),

- progressive Erschöpfung,

- Atem- oder Herzkreislaufstillstand.

Eine Erhöhung des $\mathrm{pCO}_{2}$ allein ist keine Indikation für eine endotracheale Intubation [687]. Behandeln Sie den Patienten, nicht die Zahlen. Alle Patienten, die auf eine Intensivstation verlegt werden, sollen von einem Arzt begleitet werden, der ausgestattet und in der
Lage ist, eine endotracheale Intubation durchzuführen.

\section{Nicht invasive Beatmung}

Die Anwendung nicht-invasiver Beatmung (NIV) verringert die Intubationsrate sowie die Mortalität bei COPD [688], allerdings ist die Rolle des NIV bei Patienten mit schwerem akutem Asthma ungeklärt, da es keine ausreichenden Beweise für ihre routinemäßige Anwendung bei Asthma gibt [689].

\section{Behandlung des Kreislaufstill- stands}

\section{Basismaßnahmen - „basic life support“ (BLS)}

Führen Sie BLS gemäß Leitlinienstandard durch. Eine Beatmung wird aufgrund des erhöhten Atemwegswiderstands erschwert sein; eine Luftinsufflation in den Magen ist zu vermeiden.

\section{Erweiterte Maßnahmen - „advanced} life support" (ALS)

Eine Anpassung an die Standard-ALSLeitlinien betrifft die Notwendigkeit einer frühzeitigen Intubation. Die Beatmungsdrücke während der Beatmung von Patienten mit schwerem Asthma (Mittelwert 67,8 $\pm 11,1 \mathrm{cmH}_{2} \mathrm{O}$ bei 12 Patienten) sind deutlich höher als die normalen Drücke des unteren Ösophagussphinkters (ca. $20 \mathrm{cmH}_{2} \mathrm{O}$, [690]). Es gibt ein erhebliches Risiko von Luftinsufflation in den Magen und Hypoventilation der Lunge beim Versuch, einen Patienten mit schwerem Asthma ohne Endotrachealtubus zu beatmen. Während eines Kreislaufstillstands ist dieses Risiko noch höher, weil der Druck im unteren Ösophagussphinkter noch wesentlich geringer ist als normal [691].

Atemfrequenzen von 8-10 Atemzügen/min und ein Atemvolumen für eine normale Thoraxbewegung während der CPR sollen das Risiko einer dynamischen Überblähung der Lunge („air-trapping“) minimieren, wobei das Atemvolumen von der Inspirationszeit und dem Inspirationsflow, die Lungenentleerung von Exspirationszeit und -flow abhängt. Bei beatmeten Patienten mit schwerem Asthma führt eine Erhöhung der Exspirationszeit (bewirkt 
durch Reduzierung der Atemfrequenz) zu einer nur geringen Verbesserung mit geringerem „air trapping“, wenn ein Minutenvolumen von $<10 \mathrm{l} / \mathrm{min}$ verwendet wird [690].

Einige Fallberichte fanden ROSC bei Patienten mit „air trapping“, wenn der Trachealtubus diskonnektiert wurde [692-696]. Besteht der Verdacht auf eine dynamische Überblähung der Lunge während der CPR, so können Thoraxkompressionen bei diskonnektiertem Trachealtubus das "air trapping“ reduzieren. Obwohl dieses Verfahren nur durch begrenzte Evidenz belegt ist, ist eine weitere Patientenschädigung in diesen ansonsten hoffnungslosen Situationen nicht zu erwarten.

Eine Hyperinflation erhöht die transthorakale Impedanz, aber moderne impedanzkompensierte biphasische Defibrillationsformen sind bei diesen Patienten nicht weniger wirksam [697]. Wie beim ALS-Defibrillationsstandard ist eine Steigerung der Defibrillationsenergie zu erwägen, wenn der erste Schock nicht erfolgreich ist und ein manueller Defibrillator zur Verfügung steht.

Es gibt keine guten Hinweise für den Einsatz der offenen Thoraxkompression bei Patienten mit asthmaassoziiertem Kreislaufstillstand. Eine Abarbeitung der 4 Hs und HITS ermöglicht die Identifizierung potenziell reversibler Ursachen von asthmabezogenen Kreislaufstillständen. Die Diagnose eines Spannungspneumothorax ist unter CPR möglicherweise erschwert: Hinweise können eine einseitige Bewegung der Thoraxwand bei Ventilation, eine Trachealverlagerung und/oder ein Hautemphysem sein. Die Sonographie der Pleura durch einen erfahrenen Untersucher ist schneller und sensitiver zum Pneumothoraxnachweis als der Röntgenthorax [698]. Wenn ein Pneumothorax vermutet wird, soll eine Dekompression mit einer großlumigen Kanüle durchgeführt werden, wobei eine direkte Punktion der Lunge zu vermeiden ist. Jedem Versuch einer Nadeldekompression soll die Anlage einer Thoraxdrainage folgen. Bedenken Sie immer die Möglichkeit eines bilateralen Pneumothorax in asthmaverursachtem Kreislaufstillstand (s. „Spannungspneumothorax“).
ECLS kann sowohl die Organdurchblutung als auch den Gasaustausch in Fällen eines fortbestehenden Atem- und Kreislaufversagens sicherstellen. Es wurde von Fällen der erfolgreichen Behandlung von asthmaassoziiertem Kreislaufstillstand bei Erwachsenen mit ECLS berichtet $[699,700]$. Allerdings wurde die Rolle der ECLS in diesem Zusammenhang nie in kontrollierten Studien untersucht. Die Verwendung von ECLS erfordert eine entsprechende Ausrüstung sowie entsprechend notwendige Fertigkeiten, die möglicherweise nicht allen Krankenhäusern zur Verfügung stehen.

\section{Patienten mit Herzunterstützungssystemen}

\section{Einführung}

Alle Ärzte, die Patienten mit Herzunterstützungssystemen (VAD) betreuen, sollen umfassend geschult sein, geräteseitige Probleme zu beherrschen und mit Kreislaufstillstand bei diesen Patienten umzugehen. Die Behandlung von Patienten mit VAD ist komplex, da der Kreislaufstillstand durch technisches, mechanisches Versagen ausgelöst sein kann, welches ein gerätespezifisches Vorgehen erfordert. Die Verwendung von Thoraxkompressionen bei Patienten mit Herzunterstützungssystemen wurde ausgewertet, wobei Einzelfallberichte über erfolgreiche CPR ohne Beschädigung des VAD existieren [701]. Thoraxkompressionen können insbesondere hilfreich sein, um einen nicht funktionalen rechten Ventrikel zu entlasten, da dieser oftmals ursächlich für den Verlust des Auswurfs ist.

\section{Diagnose des Kreislaufstillstands}

Die Bestätigung des Kreislaufstillstands kann bei diesen Patienten schwierig sein. Bei Patienten unter invasivem Monitoring soll ein Stillstand in Betracht gezogen werden, wenn die arterielle Druckmessung dieselben Werte wie die zentrale Venendruckmessung (CVP) anzeigt. Bei Patienten ohne invasives Monitoring ist ein Kreislaufstillstand anzunehmen, wenn keine Lebenszeichen vorliegen und keine Atmung vorhanden ist. Transthorakale/ transösophageale Echokardiographie (TTE/TEE), Kapnographie oder Doppler- flussmessungen in den Hauptarterien können sinnvoll sein, um die Perfusion einzuschätzen. Herzunterstützungssysteme zeigen auch den Pumpendurchfluss an; dies soll zur Einschätzung genutzt werden, ob ein echter Blutverlust vorliegt oder ob es sich nur um eine Low-flow-Situation mit eingeschränktem Bewusstseinszustand handelt.

\section{Management des Kreislaufstill- stands}

Bei Patienten mit einem implantierbaren linksventrikulären Unterstützungssystem (LVAD), wie HeartMate (Thoratec, Pleasanton, CA, USA) oder HeartWare (Heartware, Framingham, MA, USA) soll dem Algorithmus gefolgt werden, der auch für CPR nach einer Herzoperation gilt (s. „Kreislaufstillstand nach Herzoperationen"). Überprüfen Sie den Rhythmus, führen sie ggf. eine Defibrillation bei defibrillierbaren Rhythmen (VF/pVT) durch bzw. beginnen Sie mit einem Pacing bei Asystolie. Bei pulsloser elektrischer Aktivität (PEA), schalten Sie den Schrittmacher aus, und bestätigen Sie, dass es kein zugrunde liegendes VF gibt, welches defibrilliert werden muss. Thoraxkompressionen sollen begonnen werden, wenn diese sofortige Reanimationsmaßnahmen fehlgeschlagen sind. Eine Kontrolle der Atemwege und der Atmung muss immer erfolgen.

Es ist möglich, dass ein Patient eine Asystolie oder VF aufweist, aber dennoch eine ausreichende Hirndurchblutung aufgrund adäquater bestehender Pumpenleistung hat. Ist der Patient bei Bewusstsein und reagiert, dann steht mehr Zeit zur Behebung der Rhythmusstörungen zur Verfügung, und Thoraxkompressionen werden nicht benötigt.

Eine Resternotomie soll bei Kreislaufstillstand innerhalb von 10 Tagen nach der Operation durchgeführt werden, nach dieser Zeit stellt die Resternotomie oder der Einsatz der extrakorporaler Membranoxygenierung (ECMO) eine Option dar. 


\section{Kreislaufstillstand assoziiert mit neurologischen Krankheitsbildern}

\section{Ursache des Kreislaufstillstand}

Kreislaufstillstand bei neurologischen Krankheitsbildern ist relativ selten, er kann bei Subarachnoidalblutung, intrazerebraler Blutung, Krampfanfällen oder ischämischem Schlaganfall auftreten [702]. Zudem können Hirnverletzungen nach Trauma zum Kreislaufstillstand führen.

Kreislaufstillstand, assoziiert mit neurologischen Krankheitsbildern, kann durch folgende Ursachen bedingt sein:

- Verlust des Bewusstseins mit nachfolgender Atemwegsverlegung, Hypoxämie und Atemstillstand, gefolgt von Kreislaufstillstand. Bewusstseinsverlust ist zudem mit einem erhöhten Aspirationsrisiko verbunden,

- Atemdepression und Kreislaufdekompensation aufgrund einer Kompression des Hirnstamms,

- Herzrhythmusstörungen und myokardiale Dysfunktion als Folge akuter neurologischer Schädigung, insbesondere bei subarachnoidaler Blutung,

- der sog. „sudden unexpected death in epilepsy“ (SUDEP) betrifft etwa einen von 1000 Epileptikern [703].

\section{Neurologische Symptome}

Die Patienten können Prodromalanzeichen aufweisen, die auf eine neurologische Ursache vor Kreislaufstillstand hindeuten, wie z. B. Kopfschmerzen, Krampfanfälle, Bewusstseinsstörungen oder fokale Zeichen, wobei diese unspezifisch sind und zudem mit Synkopen, Atemnot und Thoraxschmerz assoziiert sein können [704]. Ein Kreislauf- oder Atemstillstand tritt in 3-11\% der Patienten mit Subarachnoidalblutung auf [705]. Bei dem initialen Rhythmus handelt es sich in der Regel um einen nicht-schockbaren Rhythmus.

\section{Behandlung}

Maßnahmen zur Vorbeugung eines Kreislauf- oder Atemstillstands sollen auf die Behandlung reversibler Ursachen fokussieren. Tritt ein Kreislaufstillstand ein, sind die Standardempfehlungen zu BLS und ALS zu befolgen. Sollte ein
ROSC erreicht werden, dann sind neben der Standardpostreanimationstherapie die zugrunde liegenden Ursachen zu berücksichtigen.

Patienten mit Subarachnoidalblutung können EKG-Veränderungen aufweisen, die auf ein akutes Koronarsyndrom hinweisen [704, 706]. Bestimmte Merkmale wie jung, weiblich, initial nicht-schockbarer Rhythmus und eine neurologische Vorgeschichte (z. B. Kopfschmerzen, Krampfanfälle, neurologische Defizite) sind häufig, aber unspezifisch für neurologische Ursachen [707]. Bei Patienten mit neurologischen Prodromalsymptomen, bei denen ein ROSC erreicht werden konnte, soll an eine Schädel-CT-Untersuchung gedacht werden. Ob diese vor oder nach Koronarangiographie durchgeführt wird, muss anhand der klinischen Einschätzung unter Abwägung der Wahrscheinlichkeit einer Subarachnoidalblutung zum akuten Koronarsyndrom als Ursache entschieden werden.

\section{Outcome}

Das Überleben hängt von den zugrunde liegenden Ursachen und den üblichen Faktoren (z. B. beobachtet oder nicht, Notfallzeugen-CPR) für Überleben ab [702]. Eine schlechte Prognose haben Patienten mit ROSC nach Subarachnoidalblutung [704, 706, 708]. Patienten, die nach einer primären neurologischen Ursache bei Kreislaufstillstand einen ROSC erreichen, erfüllen oft neurologische Kriterien für einen Hirntod und sollen als potenzielle Organspender berücksichtigt werden [709].

\section{Adipositas}

\section{Einführung}

Weltweit hat sich Fettleibigkeit seit 1980 mehr als verdoppelt. Im Jahr 2014 waren über 1,9 Mrd. (39\%) Erwachsene übergewichtig und von diesen mehr als 600 Mio. (13\%) adipös.

Die WHO definiert Übergewicht bei Erwachsenen mit Body-Mass-Index (BMI, $\mathrm{kg} / \mathrm{m}^{2}$ ) wie folgt: [710-712]

- Übergewicht $\left(25,0-29,9 \mathrm{~kg} / \mathrm{m}^{2}\right)$,

- adipös $\left(30,0\right.$ bis $\left.34,9 \mathrm{~kg} / \mathrm{m}^{2}\right)$,

- sehr adipös $\left(\geq 35,0 \mathrm{~kg} / \mathrm{m}^{2}\right)$.
Viele klinische Studien haben einen $\mathrm{Zu}$ sammenhang zwischen BMI und einer Vielzahl von kardiovaskulären und nicht kardiovaskulären Zuständen gefunden [713-715]. Traditionelle kardiovaskuläre Risikofaktoren (Bluthochdruck, Diabetes, Lipidprofil, koronare Herzerkrankung, Herzinsuffizienz und linksventrikuläre Hypertrophie) sind häufig bei adipösen Patienten. Adipositas ist mit einem erhöhten Risiko des plötzlichen Herztods assoziiert [715]. Häufigste Todesursachen sind dilatative Kardiomyopathie und schwere koronare Atherosklerose [716].

\section{Änderungen bei der kardio- pulmonalen Reanimation}

Es werden keine Änderungen hinsichtlich der Abfolge der Maßnahmen bei der Reanimation von adipösen Patienten empfohlen, wobei die adäquate Durchführung der CPR eine Herausforderung sein kann. Physikalische und physiologische Faktoren im Zusammenhang mit Adipositas können sich negativ auf die CPR auswirken, einschließlich Patientenzugang und Transport, Patientenbeurteilung, schwieriger i.v.Zugang, Atemwegsmanagement, Qualität der Thoraxkompression, Wirksamkeit vasoaktiver Medikamente sowie Effizienz der Defibrillation, weil keine dieser Maßnahmen anhand des BMI oder des Gewichts der Patienten standardisiert ist [710]. Mehr Helfer als sonst können erforderlich sein, um beim Transport des Patienten $\mathrm{zu}$ assistieren, und die Ermüdung der Helfer, insbesondere bei der Thoraxkompression, soll durch häufigere Helferwechsel vermieden werden.

\section{Thoraxkompression}

Wie bei jedem Kreislaufstillstand sind Thoraxkompressionen am effektivsten, wenn der Patient auf einer festen Unterlage liegt. Jedoch kann es für ihn und den Helfer ggf. unsicher sein, wenn der Patient hingelegt wird. Bei adipösen Patienten ist das jedoch auch nicht immer notwendig, da der schwerere Torso in die Matratze einsinkt, sodass eine geringere Wahrscheinlichkeit für eine Matratzenverlagerung während der Thoraxkompression besteht [717].

Um eine ausreichende Kompressionstiefe (ca. $5 \mathrm{~cm}$, aber nicht mehr als $6 \mathrm{~cm}$ ) 
$\mathrm{zu}$ erreichen, ist ein höherer Kraftaufwand nötig, der schneller zur Ermüdung der Helfer führen und einen häufigeren Helferwechsel als das Standard-2-minIntervall erforderlich machen kann. Die Verwendung mechanischer Reanimationsgeräte ist durch die Neigung der vorderen Brustwand, die Brustabmessungen und das Patientengewicht begrenzt [maximale Brustbeinhöhe bis $303 \mathrm{~mm}$ und maximale Breite bis $449 \mathrm{~mm}$ für das Stempelgerät (LUCAS), maximaler Brustumfang bis $130 \mathrm{~cm}$ und maximale Breite der Brust $380 \mathrm{~mm}$ für das „Load-distributing-band-Gerät“ (AutoPulse 2013), maximales Gewicht bis $136 \mathrm{~kg}$ (AutoPulse 2013)].

\section{Defibrillation}

Optimale Defibrillationsenergien bei adipösen Patienten sind nicht bekannt. Anders als monophasische Defibrillatoren sind moderne biphasische impedanzkompensiert und steuern ihre abgegebene Energie nach der Impedanz des Patienten. Zwei kleine retrospektive Studien haben keine gewichtsabhängige Einflüsse auf die Wirksamkeit der Defibrillation gefunden [718], mit einer zweiphasigen Wellenform von $150 \mathrm{~J}$ konnte eine hohe Schockerfolgsrate ohne Energiesteigerung erzielt werden [719]. Defibrillationsprotokolle für adipöse Patienten sollen daher den Empfehlungen für Patienten mit normalem BMI folgen. Höhere Defibrillationsenergien sollen bedacht werden, wenn anfängliche Versuche fehlschlagen.

\section{Ventilation}

Höhere Inspirationsdrücke sind für die Überdruckbeatmung wegen des erhöhten intraabdominellen Druckes erforderlich [720]. Eine frühzeitige endotracheale Intubation durch erfahrene Anwender erspart längere Beutel-MaskenBeatmung und kann jede Gefahr einer Aspiration reduzieren. Bei allen Patienten mit extremer Adipositas ist mit einer schwierigen Intubation zu rechnen, und ein klares Vorgehen bei gescheitertem Intubationsversuch ist notwendig [721]. Bei nicht erfolgreicher Intubation ist die Verwendung eines supraglottischen Luftwegs (SAD) mit ösophagealem Drainagekanal eine geeignete Option.

\section{Logistische Überlegungen}

Der BMI eines Patienten soll bei der Organisation der prähospitalen Reanimation berücksichtigt werden, insbesondere im Hinblick auf den technischen Support und die Anzahl des Rettungsfachpersonals [722]. Sonderfahrzeuge zum Transport extrem übergewichtiger Patienten sollen wenn möglich verwendet werden, z. B. mit extrabreitem Interieur, verstärkten Tragen und Spezialhebevorrichtungen. Gewichtsgrenzen der Krankentragen und Krankenbetten müssen vorher überprüft werden [723]. Eine Unterschätzung der technischen Aspekte der Rettungsmaßnahmen kann sekundäre Transporttraumata verursachen oder sogar den sicheren Transfer von adipösen Patienten zum Krankenhaus verhindern [722].

\section{Outcome}

Der Zusammenhang zwischen Übergewicht und dem Outcome bei Kreislaufstillstand ist unklar. Eine große Registerstudie hat gezeigt, dass Überleben von Kreislaufstillstand bei Vorliegen von defibrillierbaren Rhythmen (VF/pVT) am höchsten bei übergewichtigen Patienten war, jedoch signifikant niedriger bei sehr adipösen Patienten [710]. Im Gegensatz dazu war bei nicht defibrillierbaren Rhythmen die Überlebensrate bei Entlassung in allen BMI-Gruppen vergleichbar. Hinweise aus klinischen Kohortenstudien deuten darauf hin, dass übergewichtige und adipöse Patienten tatsächlich eine günstigere kurzfristige und langfristige Prognose haben als schlanke, sobald sie erfolgreich wiederbelebt wurden $[711,724]$.

\section{Kreislaufstillstand in der Schwangerschaft}

\section{Einführung}

Die Sterblichkeit im Zusammenhang mit Schwangerschaft ist in Europa relativ gering (Schätzung: 16 pro 100.000 Lebendgeburten), obwohl es große Unterschiede zwischen den Ländern gibt [725]. Der Fötus muss immer berücksichtigt werden, wenn ein unerwünschtes kardiovaskuläres Ereignis bei einer schwangeren Frau auftritt. Das fetale Überleben ist in der Regel abhängig vom Überleben der
Mutter, und die initialen Reanimationsbemühungen sollen sich somit auf die schwangere Patientin konzentrieren. Wiederbelebungsempfehlungen bei Vorliegen einer Schwangerschaft basieren weitgehend auf Fallserien, Extrapolation von Kreislaufstillständen bei Nichtschwangeren, Simulationsstudien und Expertenmeinungen auf der Grundlage der Physiologie der Schwangerschaft und der Änderungen, die bei einer normalen Geburt auftreten [726, 727].

Während der Schwangerschaft treten signifikante physiologische Veränderungen auf (z. B. Herzzeitvolumen, Blutvolumen, Atemminutenvolumen), und der Sauerstoffverbrauch steigt generell an. Darüber hinaus kann der schwangere Uterus eine erhebliche Kompression der Becken- und Bauchgefäße verursachen, wenn die Mutter sich in Rückenlage befindet, was zu einer verringerten Herzleistung und Blutdruckabfall führt.

\section{Ursachen des Kreislaufstillstands}

In entwickelten Regionen sind Blutungen, Embolien (thromboembolisch oder durch Fruchtwasser), Hypertonie während der Schwangerschaft, Aborte oder Urogenitalsepsis für die meisten Todesfälle verantwortlich, die unmittelbar mit der Schwangerschaft verbunden sind. Vorbestehende Erkrankungen sind für die verantwortlich, die indirekt mit einer Schwangerschaft in Verbindung stehen [728]. Ein Review zu über 2 Mio. Schwangerschaften in Großbritannien zeigte, dass die Müttersterblichkeit (Tod während Schwangerschaft, Geburt oder innerhalb von 42 Tagen nach Geburt) mit Herzerkrankungen, neurologischen und psychiatrischen sowie Tumorerkrankungen assoziiert ist [729]. Ein Viertel der schwangeren Frauen, die in Großbritannien starben, hatte eine Sepsis und eine von 11 Frauen hatte Grippe. Schwangere Frauen können zudem einen Kreislaufstillstand aus den gleichen Ursachen erleiden wie nicht schwangere Frauen derselben Altersgruppe.

\section{Prävention von Kreislaufstillstand in der Schwangerschaft}

Verwenden Sie im Notfall das systematische ABCDE-Schema. Viele 
Herz-Kreislauf-Probleme, die mit der Schwangerschaft in Verbindung stehen, sind durch aortocavale Kompression bedingt.

Behandeln Sie eine schwangere Patientin wie folgt:

- Bringen Sie die Patienten in die Linksseitenlage oder verschieben Sie von Hand sanft den Uterus nach links.

- Geben Sie Sauerstoff, orientiert an der Pulsoximetrie, um jede Hypoxämie zu korrigieren.

- Geben Sie einen Flüssigkeitsbolus, wenn eine Hypotonie oder Hypovolämie vorliegt.

- Bewerten Sie die Notwendigkeit zur Medikamentengabe regelmäßig.

- Ziehen Sie frühzeitig Experten zu Rate. Geburtshilfliche und neonatale Spezialisten sollen bei Reanimationen frühzeitig beteiligt werden.

- Identifizieren und behandeln Sie zugrunde liegende Ursachen, $z$. B. schnelle Erkennung und Behandlung von Sepsis, einschließlich frühzeitiger intravenöser Gabe von Antibiotika.

\section{Änderungen beim "basic life support"}

Ab der 20. Schwangerschaftswoche (SSW) kann der Uterus sowohl die untere Hohlvene als auch die Aorta komprimieren und so den venösen Rückfluss und das Herzzeitvolumen beeinträchtigen. Eine Behinderung des venösen Rückstroms des Uterus kann Pre-arrest-Hypotonie oder Schock verursachen bzw. in kritisch kranken Patienten einen Kreislaufstillstand auslösen [730, 731]. Während eines solchen begrenzt die Beeinflussung des venösen Rückstroms und des „cardiac output" durch den schwangeren Uterus die Effektivität der Thoraxkompressionen.

Nicht-arrest-Studien zeigen, dass die Linksseitenlagerung den mütterlichen Blutdruck, das Herzminuten- und Schlagvolumen [732-734] und die Sauerstoffversorgung sowie die fetale Herzfrequenz verbessert [735-737]. Diese Daten zeigen, dass der schwangere Uterus meistens manuell von der V. cava inferior verschoben werden kann, indem die Patientinnen $15^{\circ}$ in Linksseitenlage positioniert werden [738]. Der Wert der Reduzierung der Aorten- oder V.-cava-
Kompression während CPR ist jedoch unbekannt. Solange sich die schwangere Patientin nicht auf einem kippbaren OP-Tisch befindet, ist die Herstellung einer Linksseitenlage nicht einfach, wenn gleichzeitig eine hohe Qualität der Thoraxkompressionen gewährleistet sein soll. Eine Vielzahl von Verfahren zur Linksseitenlage sind beschrieben, einschließlich der Positionierung der Patientin auf den Knien des Helfers [739], Kissen oder Decken oder des sog. „cardiff wedge“, obwohl deren Wirksamkeit bei tatsächlichen Kreislaufstillständen nicht bekannt ist [740]. Selbst wenn ein Schwenktisch verwendet wird, so wird der Neigungswinkel oft überschätzt [741]. In einer Simulationsstudie verringerte sich die Möglichkeit, effektive Thoraxkompressionen durchzuführen, mit der Zunahme der Linksseitenlage, wobei das Simulationsmodell ab einem Winkel von $>30^{\circ}$ dazu neigte wegzurollen [740].

Die wichtigsten Schritte für BLS bei einer schwangeren Patientin sind:

- Ziehen Sie frühzeitig einen Experten hinzu (einschließlich Geburtshelfer und Neonatologen).

- Beginnen Sie BLS nach den Standardempfehlungen.

- Stellen Sie qualitativ hochwertige Thoraxkompressionen mit minimalen Unterbrechungen sicher.

- Die Handposition für die Thoraxkompression muss bei Patientinnen mit fortgeschrittener Schwangerschaft (z. B. im 3. Trimester) möglicherweise etwas höher auf dem Brustbein gewählt werden [726].

- Verdrängen Sie die Gebärmutter manuell nach links, um eine V.-cavaKompression zu verringern.

- Führen Sie, wenn dies möglich und sicher ist, eine Seitenneigung nach links durch, und stellen Sie sicher, dass der Brustkorb auf einer festen Unterlage bleibt (z. B. im Operationssaal) - der optimale Neigungswinkel ist nicht bekannt, Ziel ist ein Wert zwischen 15 und $30^{\circ}$. Selbst eine geringe Neigung kann besser sein als gar keine. Der verwendete Neigungswinkel muss eine qualitativ hochwertige Thoraxkompression und, wenn nötig, einen Kaiserschnitt ermöglichen.
- Beginnen Sie mit der Vorbereitung für einen Notfallkaiserschnitt (s. unten) - der Fetus muss entbunden werden, wenn anfängliche Wiederbelebungsbemühungen scheitern.

\section{Änderungen beim „advanced life support ${ }^{\prime \prime}$}

\section{Defibrillation}

Bei Vorliegen eines defibrillierbaren Rhythmus (VF/pVT) soll sobald wie möglich defibrilliert werden. Es gibt keine Änderung der transthorakalen Impedanz während der Schwangerschaft, sodass Standardenergien für Defibrillationsversuche verwendet werden [742]. Ebenfalls fehlen Hinweise darauf, dass Schocks mit einem (Gleichstrom-)Defibrillator negative Auswirkungen auf das fetale Herz haben.

\section{Atemwegsmanagement}

Während der Schwangerschaft gibt es ein größeres Risiko einer Insuffizienz des gastroösophagealen Sphinkter mit der Aspiration von Mageninhalt [743, 744]. Obwohl schwangere Patientinnen ein erhöhtes Aspirationsrisiko haben, hat die Sauerstoffversorgung und Beatmung eindeutig Vorrang vor der Aspirationsprophylaxe. Eine frühe endotracheale Intubation erleichtert die Belüftung der Lunge bei gleichzeitig erhöhtem intraabdominellem Druck.

Ein Trachealtubus mit einem Innendurchmesser (ID) 0,5-1,0 mm, kleiner als für eine nicht schwangere Frau von ähnlicher Größe, kann bei Verengung der mütterlichen Atemwege aufgrund von Ödem und Schwellung notwendig sein [745]. Eine Studie konnte zeigen, dass die oberen Atemwege im dritten Trimester enger sind als postpartal und bei nicht schwangeren Kontrollpatientinnen [746]. Die endotracheale Intubation kann bei schwangeren Patientinnen schwieriger sein [747]. Expertenhilfe, Training der schwierigen Intubation und der Einsatz alternativer Atemwegssicherung kann notwendig werden [748].

\section{Intravenöser Zugang}

Ein früher i.v.- oder i.o.-Zugang ermöglicht eine Medikamenten- und Flüssig- 
keitsapplikation. Der Versuch einer Anlage oberhalb des Zwerchfells berücksichtigt theoretische Bedenken zu einer verzögerten Zirkulation, verursacht durch eine V.-cava-Kompression, wenn Medikamente von Zugängen unterhalb des Zwerchfells infundiert werden.

\section{Reversible Ursachen}

Häufige und reversible Ursachen für Kreislaufstillstand in der Schwangerschaft sollen identifiziert werden (s. „Besondere Ursachen“). Die 4 Hs und HITS helfen bei der Identifizierung aller häufigen Ursachen von Kreislaufstillstand in der Schwangerschaft. Schwangere Patienten können gemäß ihrer Altersgruppe auch aus anderen Gründen, die nichts mit der Schwangerschaft zu tun haben, (z. B. Anaphylaxie, Medikamentenüberdosierung, Trauma) einen Kreislaufstillstand zu erleiden.

Ziehen Sie die Verwendung von Abdominalultraschall durch einen erfahrenen Untersucher in Betracht, um mögliche Ursachen für einen Kreislaufstillstand zu erkennen; aber verzögern Sie nicht andere Behandlungen, und minimieren Sie die Unterbrechungen bei den Thoraxkompressionen.

Spezifische Ursachen für Kreislaufstillstand in der Schwangerschaft sind:

\section{Blutungen}

Lebensbedrohliche Blutungen können sowohl prä- als auch postpartal auftreten [728]. Postpartale Blutungen sind die häufigste Einzelursache für die Müttersterblichkeit weltweit, und man schätzt, dass alle 7 min eine Mutter daran verstirbt [749]. Ursächlich können hier Eileiterschwangerschaft, Plazentalösung, Placenta praevia, Placenta accreta oder eine Uterusruptur sein [750]. Generell soll ein Protokoll zum Vorgehen bei Massivtransfusion verwendet und in Verbindung mit der Blutbank regelmäßig aktualisiert werden. Schwangere mit hohem Blutungsrisiko sollen in Zentren mit Einrichtungen für Bluttransfusion und Intensivmedizin entbinden, und Vorkehrungen hierfür sollen im Voraus geplant werden. Die Behandlung basiert auf dem ABCDE-Schema. Entscheidend ist dabei, die Blutung zu stoppen.

Denken Sie an [751, 752]:
- Volumentherapie, einschließlich der Verwendung von schnellen Transfusions- und Blutaufbereitungssystemen [753],

- Oxytocin und Prostaglandin-Analoga zur Behandlung von Uterusatonie [754],

- Massage des Uterus [755],

- Korrektur einer Gerinnungsstörung, einschließlich der Verwendung von Tranexamsäure und/oder rekombinantem aktiviertem Faktor VII [756-758],

- Uterus-Ballon-Tamponade oder Packing [759, 760],

- Uteruskompressionsnähte [761],

- Angiographie und endovaskuläre Embolisation [762],

- Hysterektomie [763, 764],

- aortale Abklemmung bei extremer Hämorrhagie [765].

\section{Kardiovaskuläre Erkrankungen}

Herzinfarkt und Aneurysmen oder Dissektionen der Aorta oder ihrer Abgänge sowie peripartale Kardiomyopathien verursachen die meisten Todesfälle bei erworbenen Herzerkrankungen [766768]. Patientinnen mit bekannter Herzerkrankung sollen in einer Fachabteilung behandelt werden. Schwangere Frauen können ein akutes Koronarsyndrom in der Regel in Verbindung mit Risikofaktoren wie Übergewicht, Alter, zunehmender Dauer der Schwangerschaft, Rauchen, Diabetes, bereits bestehendem Bluthochdruck und einer Familiengeschichte ischämischer Herzerkrankungen entwickeln [750, 769]. Sie können atypische Symptome wie Oberbauchschmerzen und Erbrechen aufweisen. Perkutane Koronarintervention (PCI) ist die Reperfusionsstrategie der Wahl für den ST-Hebungsinfarkt in der Schwangerschaft. Thrombolyse soll berücksichtigt werden, wenn eine dringend erforderliche PCI nicht verfügbar ist. Eine Auswertung von 200 Fällen von Thrombolyse bei massiver Lungenembolie in der Schwangerschaft zeigte eine Müttersterblichkeitsrate von 1\% und schlussfolgerte, dass die Thrombolyse relativ sicher in der Schwangerschaft ist [770].

Eine wachsende Zahl von Frauen mit angeborenen Herzfehlern werden schwanger [771]. Herzinsuffizienz und Herzrhythmusstörungen sind die häufigsten Probleme, v. a. bei Patienten mit zyanotischen Herzerkrankungen. Schwangere Frauen mit bekannten angeborenen Herzfehlern sollen in spezialisierten Zentren betreut werden.

\section{Präeklampsie und Eklampsie}

Eklampsie ist definiert als das Auftreten von Krampfanfällen und/oder eines unerklärlichen Komas bei Patienten mit Anzeichen und Symptomen einer Präeklampsie während der Schwangerschaft oder der Geburt [772, 773]. Magnesiumsulfat ist in etwa der Hälfte der Fälle von Eklampsie während der Geburt oder unmittelbar nach der Geburt bei Frauen mit Präeklampsie effektiv [774-777]. Verwenden Sie Magnesiumsulfat als Infusion zur Behandlung von Eklampsie [778-781].

\section{Lungenembolie}

Die geschätzte Inzidenz von Lungenembolie liegt bei 1,0$1,5 / 10.000$ Schwangerschaften mit einer Letalität von 3,5\% (95\%-KI=1,1-8,0\%, [782]). Zu den Risikofaktoren zählen Übergewicht, höheres Alter sowie Immobilisation. Erfolgreicher Einsatz von Fibrinolytika für massive, lebensbedrohliche Lungenembolien bei schwangeren Frauen ist dokumentiert [770, 783-786].

\section{Fruchtwasserembolie}

Eine Fruchtwasserembolie („amniotic fluid embolism“, AFE) tritt in der Regel um den Zeitpunkt der Geburt herum mit plötzlichem Kreislaufkollaps, Atemnot, Zyanose, Arrhythmien, Hypotonie und Blutung mit disseminierter intravasaler Koagulopathie auf [787]. Patientinnen können Warnzeichen wie Atemnot, Brustschmerzen, Kältegefühl, Benommenheit, Angst, Panik, Kribbelparästhesien in den Fingern, Übelkeit oder Erbrechen zeigen. Das „united kingdom obstetric surveillance system" (UKOSS) identifizierte 120 Fälle von AFE zwischen 2005 und 2014 mit einer ungefähren Gesamtletalität von 1,7 und 0,3/100.000 Einwohner, assoziiert mit höherem Alter der Mutter, Mehrlingsschwangerschaft, Placenta praevia und 
Einleitung der Geburt, instrumentierte vaginale Geburt oder Kaiserschnitt [788].

Die Behandlung ist supportiv, da es keine spezifische Therapie auf der Basis des ABCDE-Schemas oder der Korrektur einer Koagulopathie gibt. Der erfolgreiche Einsatz der extrakorporalen Unterstützung nach lebensbedrohlicher Fruchtwasserembolie während der Wehen und der Geburt ist beschrieben [789].

\section{Perimortale Entbindung des Fötus}

Ziehen Sie die Notwendigkeit einer Notfallhysterotomie oder eines Kaiserschnitts in Betracht, sobald eine Schwangere einen Kreislaufstillstand erleidet. In einigen Fällen führen sofortige Wiederbelebungsversuche zur Wiederherstellung der Perfusion; in der Frühschwangerschaft kann dies die Fortführung der Schwangerschaft ermöglichen. Drei Beobachtungsstudien mit insgesamt 154 Probanden zeigen Evidenz mit sehr geringer Qualität für die Verwendung von perimortalem Kaiserschnitt [790-792]. Basierend auf Expertenmeinungen kann eine Entbindung des Fötus, wenn anfängliche Wiederbelebungsversuche scheitern, die Chancen einer erfolgreichen Wiederbelebung der Mutter und des Fötus erhöhen [793-795]. Ein systematische Übersicht zeigte 38 Fälle von Kaiserschnitt während CPR, mit 34 überlebenden Säuglingen und 13 Müttern, die das Krankenhaus lebend verließen, was darauf hindeutet, dass Kaiserschnitt das Outcome von Müttern und Neugeborenen verbessert [796]. Die beste Überlebensrate tritt für Kinder nach der 24. bis 25 . SSW auf, wenn die Geburt innerhalb von 5 min nach dem Kreislaufstillstand durchgeführt wurde [793, 797-799]. Dies erfordert, dass die Hysterotomie etwa 4 min nach Kreislaufstillstand eingeleitet werden muss. Bei höherem Gestationsalter (30-38 Wochen) ist das Überleben des Kindes möglich, auch wenn die Entbindung erst nach 5 min vom Beginn des mütterlichen Stillstands begonnen wurde [796]. Eine Fallserie deutet darauf hin, dass es häufiger zum Einsatz von Kaiserschnitt während der CPR kommt, wenn die Teams trainiert sind [791]. In dieser Serie wurde keine Geburt innerhalb von 5 min nach Beginn der Wiederbelebung durchgeführt. Acht der 12 Frauen hatten ROSC nach Entbindung, mit 2 überlebenden Müttern und 5 überlebenden Neugeborenen. Die mütterliche Letalität betrug $83 \%$, die neonatale $58 \%$ [791].

Die Entbindung behebt die V.-cavaKompression und kann die Chancen der mütterlichen Wiederbelebung verbessern. Der Kaiserschnitt ermöglicht zudem einen Zugriff auf den Säugling, sodass die Reanimation des Neugeborenen beginnen kann.

\section{Entscheidungsfindung zur Not- fallhysterotomie (Kaiserschnitt)} Der schwangere Uterus erreicht ab der 20. SSW eine Größe, die den aortocavalen Blutfluss kompromittieren kann; die fetale Überlebensfähigkeit beginnt ca. ab der 24. bis 25. Woche [800]. Tragbare Ultraschallgeräte sind in einigen Notfallabteilungen vorhanden und können (in erfahrenen Händen) bei der Bestimmung des Schwangerschaftsalters und der Kindslage unterstützen, deren Verwendung darf aber nicht die Entscheidung zur Durchführung einer Notfallhysterotomie verzögern [801].

- Bei einem Gestationsalter $<20$ Wochen braucht eine dringliche Kaiserschnittentbindung nicht berücksichtigt zu werden, da eine erhebliche Beeinträchtigung der mütterlichen Herzleistung durch einen schwangeren Uterus dieser Größe unwahrscheinlich ist.

- Bei einem Gestationsalter von ca. 2023 Wochen, ist die Initiierung einer Notfallhysterotomie zur erfolgreichen Wiederbelebung der Mutter geeignet, wobei das Überleben des Kindes in diesem Schwangerschaftsalter eher unwahrscheinlich ist.

- Bei einem Gestationsalter von etwa $\geq 24-25$ Wochen, ist die Notfallhysterotomie dazu geeignet, das Leben der Mutter und des Kindes zu retten.

\section{Postreanimationsbehandlung}

Postreanimationsbehandlung soll den Standardempfehlungen folgen. Gezieltes Temperaturmanagement (TTM) wurde sicher und wirksam in der Frühschwangerschaft unter fetalem Monitoring durchgeführt und führte zu einem günstigen mütterlichen und fetalen Outcome nach termingerechter Entbindung [802]. Implantierbare Cardioverter-Defibrillatoren (ICD) wurden bei Patientinnen in der Schwangerschaft verwendet [803].

\section{Vorbereitung für den Kreislaufstillstand in der Schwangerschaft}

ALS in der Schwangerschaft erfordert die Koordination der mütterlichen Reanimation, der Kaiserschnittentbindung des Feten sowie der Reanimation des Neugeborenen im Idealfall innerhalb von 5 min. Um dies zu erreichen, sollen Einrichtungen, die mit einer gewissen Wahrscheinlichkeit Kreislaufstillstände in der Schwangerschaft behandeln

- Pläne und Geräte für die Wiederbelebung von Schwangeren und Neugeborenen an Ort und Stelle haben,

- die frühzeitige Einbindung der Geburtshilfe, Anästhesie und Neugeborenenteams gewährleisten,

- regelmäßige Schulungen in geburtshilflichen Notfällen sicherstellen [804, 805].

\section{Ältere Menschen}

\section{Epidemiologie}

Mehr als $50 \%$ der Menschen, die mit OHCA in den USA wiederbelebt werden, sind 65 Jahre oder älter [806]. Die Inzidenz von Kreislaufstillstand bei älteren Menschen wird also in dem Maße ansteigen, wie die Weltbevölkerung altert. Die Inzidenz von Kreislaufstillstand nimmt mit dem Alter zu. Bei Männern ist sie beim OHCA im Alter von 80 Jahren etwa 7-mal größer als im Alter von 40 Jahren [807]. Bei Frauen über 70 Jahren liegt sie mehr als 40-mal höher als bei Frauen unter 45 Jahren. In einer Beobachtungsstudie über Kreislaufstillstand im Krankenhaus machten Patienten über 65 Jahren einen Anteil von 46\% der gesamten Krankenhauspatienten während der Studiendauer und 65\% der Kreislaufstillstände auf Station aus [808]. In dieser Studie war die Inzidenz von Kreislaufstillständen mehr als doppelt so hoch wie in der jüngeren Patientenpopulation (2,2 vs. 1,0/1000 Patientenaufnahmen). 


\section{Ursachen des Kreislaufstillstands}

Die Inzidenz von koronarer Herzkrankheit wie auch der chronischen Herzinsuffizienz nimmt mit dem Alter zu. Als Folge davon haben ältere Menschen eine erhöhte Häufigkeit von kardialen Ursachen bei Kreislaufstillstand [809]. Der Anteil der plötzlichen Todesfälle (d. h. aufgrund einer primären ventrikulären Arrhythmie) nimmt jedoch mit dem Alter ab, da parallel der Anteil der Todesfälle durch andere Herz-Kreislauf-Erkrankungen ansteigt [810]. Die Inzidenz von PEA als initial aufgezeichnetem Rhythmus steigt signifikant mit dem Alter $[809,811]$, mit einem parallelen Rückgang der Häufigkeit von defibrillierbaren Rhythmen (VF/pVT, [812]).

\section{Prävention}

Die zum Kreislaufstillstand führende Verschlechterung der Vitalfunktionen wird bei älteren Patienten im Vergleich zu jüngeren weniger genau festgehalten [813]. Klinische Zeichen akut lebensbedrohlicher Zustände wie Sepsis [814], akuter Myokardinfarkt [815] oder Herzinsuffizienz [816] sind oft abgemildert oder unspezifisch bei älteren Patienten, was in weniger physiologischen Veränderungen und einer geringeren Veränderung des „Early Warning Score" (MEWS) in den $4 \mathrm{~h}$ vor Kreislaufstillstand resultiert [808].

\section{Behandlung}

\section{Management von Peri-arrest- Situationen}

Der Alterungsprozess geht mit mehreren pathophysiologischen Veränderungen einher, die bei der Behandlung von Peri-arrest-Situationen $\mathrm{zu}$ berücksichtigen sind. Zunehmendes Alter ist verbunden mit autonomer und Baroreflexdysfunktion und einer myokardialen Versteifung, die die frühdiastolische Füllung beeinträchtigt [817]. Darüber hinaus sind ältere kritisch kranke Patienten häufig hypovoläm aufgrund einer Verringerung sowohl der Flüssigkeitsaufnahme als auch der Fähigkeit zur Urinkonzentration [818]. Diese Veränderungen beeinträchtigen Herz-Kreislauf-Reaktionen auf Flüssigkeitsverlust oder Lageänderungen und erhöhen die blutdrucksenkende Wirkung von Sedativa und anderen vasoaktiven Medikamenten. Ältere Patienten haben ein erhöhtes Risiko einer schwere Hypotonie während notfallmäßiger Atemwegssicherung [819].

Vorhofflimmern ist die häufigste supraventrikuläre Rhythmusstörung bei älteren Menschen. Es verursacht oft eine kardiovaskuläre Beeinträchtigung aufgrund des Verlustes des Vorhofanteils zur diastolischen Füllung, insbesondere bei älteren Menschen, deren Ventrikelcompliance reduziert ist. Blutdruckabfall und eine erhöhte Herzfrequenz kann die Koronarperfusion reduzieren und eine kardiale Ischämie begünstigen, die in der älteren Bevölkerung mit einer höheren Inzidenz der koronaren Herzkrankheit eher vorkommt.

Ältere Patienten neigen eher dazu, Apnoe oder eine Atemdepression nach der Verabreichung von Opioiden oder Benzodiazepinen zu entwickeln [818]. Ihr geringerer Ausgangs-Sauerstoffpartialdruck erhöht auch das Risiko der Hypoxieentwicklung. Fortschreitendes Alter ist mit einer erhöhten Rate von Begleiterkrankungen assoziiert. Ältere Patienten nehmen oft diverse Medikamente ein, die mit denen der Peri-ArrestBehandlung interferieren können. Die Häufigkeit von Nebenwirkungen bei älteren Menschen ist 2- bis 3-mal höher als bei jüngeren Patienten [820].

\section{Management des Kreislaufstill- stands}

Bei der Behandlung von älteren Patienten mit Kreislaufstillstand sind keine Änderungen der Standardreanimationsempfehlungen nötig. Rettungskräften soll jedoch bewusst sein, dass die Gefahr von Sternum- sowie Rippenfrakturen größer ist [821-823]. Die Inzidenz CPRassoziierter Verletzungen steigt mit der Dauer der CPR an [823].

\section{Outcome}

Höheres Alter ist mit einer niedrigeren kurzfristigen Überlebensrate nach Kreislaufstillstand assoziiert [824-829]. In einer großen Registerstudie zu OHCA lag das Überleben bei Krankenhausentlassung bei $8 \%$ für Patienten zwischen 65 und 79 Jahre, $4 \%$ für die 80 - und $2 \%$ für die 90-Jährigen [826]. In einer weiteren
Studie lag das adjustierte Risiko für die 30-Tage-Mortalität bei wiederbelebten älteren komatösen Patienten bei 1,04 $(95 \%-\mathrm{KI}=1,03-1,06)$ pro Jahr [812].

Zunehmendes Alter ist zudem mit niedrigem langfristigem Überleben nach Reanimation verbunden. In einer retrospektiven Kohortenstudie an älteren lebend entlassenen Patienten nach CPR im Krankenhaus betrug die risikobereinigte Rate von 1-Jahres-Überleben 63,7, 58,6 und $49,7 \%$ bei Patienten von $65-74,75-$ 84 und $\geq 85$ Jahren $(p<0,001$, [827]). Eine andere Studie mit Patienten $\geq 65$ Jahre, die nach VF/pVT und CPR lebend aus dem Krankenhaus entlassen wurden, zeigte eine signifikant niedrigere langfristige Überlebensrate als vergleichbare Alters- und Geschlechtsgruppen, während dies nicht bei jüngeren wiederbelebten Patienten beobachtet wurde [830].

Bei denen, die überlebt haben, ist das neurologische Outcome bei älteren Überlebenden von Kreislaufstillständen gut: $95 \%$ haben eine "cerebral performance category" (CPC) von 1-2 zum Zeitpunkt der Entlassung aus der Intensivstation [824] und $72 \%$ bei Krankenhausentlassung [827].

\section{Entscheidung zur Wiederbelebung}

Erleiden ältere Patienten einen Kreislaufstillstand, dann haben sie eine deutlich geringere Wahrscheinlichkeit als jüngere Patienten, dass bei ihnen eine Wiederbelebung erfolgreich ist [831, 832]. Bei der Entscheidung, ältere Patienten wiederzubeleben, soll das Alter nicht als alleiniges Merkmal berücksichtigt werden, sondern weitere etablierte Kriterien, z. B. Kollaps beobachtet, Wiederbelebungszeiten und der initial dokumentierte Rhythmus als wichtige Faktoren zu Rate gezogen werden [833]. Darüber hinaus ist zu empfehlen, dass Präreanimationsfaktoren, wie der Grad an Autonomie, Lebensqualität, mentalem Status und Vorliegen schwerer Begleiterkrankungen, ebenfalls berücksichtigt werden sollen. Wann immer möglich, soll eine Entscheidung für oder gegen die Wiederbelebung im Vorfeld mit dem Patienten und seiner Familie besprochen werden (s. „Ethik der Reanimation und Entscheidungen am Lebensende“, [243]). 


\section{Korrespondenzadresse}

\section{A. Truhlár̆}

Emergency Medical Services,

Hradec Králové Region, Hradec Králové

dirks@grc-org.de

\section{Korrespondierender Übersetzer}

Dr. rer. nat. Dr. med. Burkhard Dirks

Badbergstraße 18

89075 Ulm

dirks@grc-org.de

\section{Einhaltung ethischer Richtlinien}

Interessenkonflikt. C.D. Deakin ist Direktor der Prometheus Medical Ltd; G.D. Perkins und J. Soar sind Editor der Resuscitation; G. Brattebo ist Chair der BEST foundation; $\mathrm{H}$. Brugger ist medizinischer Berater von EURAC/ICAR alpine medicine; J.P. Nolan ist Editor-in-Chief der Resuscitation; J. Dunning ist Ehrensprecher von CARDICA; J.J.L.M. Bierens ist im Board/Berater von KNRM; KNRD; Life Saving societies; K.-C. Thies ist Chair der European Trauma Course Organisation ETCO; P. Paal Ehrensprecher von Vidacare, Zoll; R. Koster ist medizinischer Berater von Physio Control and HeartSine und erhält Forschungsgelder von Physio Control, Philips, Zoll, Cardiac Science, Defibtech, Jolife; A. Truhlář, A. Alfonzo, C. Lott, C. Sandroni, D.A. Zideman, D.J. Lockey, G.E.A. Khalifa, H.M. Lossius und S. Hunyadi-Anticevic geben an, dass kein Interessenkonflikt besteht.

Dieser Beitrag beinhaltet keine Studien an Menschen oder Tieren.

\section{Literatur}

1. Soar J, Perkins GD, Abbas G et al (2010) European Resuscitation Council Guidelines for Resuscitation 2010 Section 8. Cardiac arrest in special circumstances: electrolyte abnormalities, poisoning, drowning, accidental hypothermia, hyperthermia, asthma, anaphylaxis, cardiac surgery, trauma, pregnancy, electrocution. Resuscitation 81:1400-1433

2. Safar P, Paradis NA, Weil MH (2007) Asphyxial cardiac arrest. In: Paradis NA, Halperin HR, Kern KB, Wenzel V, Chamberlain DA (Hrsg) Cardiac arrest - the science and practice of resuscitation medicine, 2. Aufl. Cambride University Ptress, Cambridge, S 969-993

3. Farmery AD, Roe PG (1996) A model to describe the rate of oxyhaemoglobin desaturation during apnoea. Br J Anaesth 76:284-291

4. DeBehnke DJ, Hilander SJ, Dobler DW, Wickman LL, Swart GL (1995) The hemodynamic and arterial blood gas response to asphyxiation: a canine model of pulseless electrical activity. Resuscitation 30:169-175

5. Deasy C, Bray J, Smith K, Bernard S, Cameron P, Committee VS (2013) Hanging-associated out-ofhospital cardiac arrests in Melbourne, Australia. Emerg Med J 30:38-42

\section{SOS-KANTO Study Group (2007)}

Cardiopulmonary resuscitation by bystanders with chest compression only (SOS-KANTO): an observational study. Lancet 369:920-926

7. Ogawa T, Akahane M, Koike S, Tanabe S, Mizoguchi T, Imamura T (2011) Outcomes of chest compression only CPR versus conventional CPR conducted by lay people in patients with out of hospital cardiopulmonary arrest witnessed by bystanders: nationwide population based observational study. BMJ 342:c7106

8. Deasy C, Bray J, Smith K, Harriss LR, Bernard SA, Cameron $P$ (2011) Paediatric hanging associated out of hospital cardiac arrest in Melbourne, Australia: characteristics and outcomes. Emerg Med J 28:411-415

9. Wee JH, Park KN, Oh SH, Youn CS, Kim HJ, Choi SP (2012) Outcome analysis of cardiac arrest due to hanging injury. Am J Emerg Med 30:690-694

10. Davies D, Lang M, Watts R (2011) Paediatric hanging and strangulation injuries: a 10-year retrospective description of clinical factors and outcomes. Paediatr Child Health 16:e78-e81

11. Penney DJ, Stewart AH, Parr MJ (2002) Prognostic outcome indicators following hanging injuries. Resuscitation 54:27-29

12. Wee JH, Park JH, Choi SP, Park KN (2013) Outcomes of patients admitted for hanging injuries with decreased consciousness but without cardiac arrest. Am J Emerg Med 31:1666-1670

13. Mahoney B, Smith W, Lo D, Tsoi K, Tonelli M, Clase C (2005) Emergency interventions for hyperkalaemia. Cochrane Database Syst Rev 2:CD003235

14. Einhorn LM, Zhan M, Hsu VD et al (2009) The frequency of hyperkalemia and its significance in chronic kidney disease. Arch Intern Med 169:1156-1162

15. Acker CG, Johnson JP, Palevsky PM, Greenberg A (1998) Hyperkalemia in hospitalized patients: causes, adequacy of treatment, and results of an attempt to improve physician compliance with published therapy guidelines. Arch Inter Med 158:917-924

16. Moranne O, Froissart M, Rossert J et al (2009) Timing of onset of CKD-related metabolic complications. J Am Soc Nephrol 20:164-171

17. Lin $\mathrm{CH}$, Tu YF, Chiang WC, Wu SY, Chang YH, Chi CH (2013) Electrolyte abnormalities and laboratory findings in patients with out-ofhospital cardiac arrest who have kidney disease. Am J Emerg Med 31:487-493

18. Khanagavi J, Gupta T, Aronow WS et al (2014) Hyperkalemia among hospitalized patients and association between duration of hyperkalemia and outcomes. Arch Med Sci 10:251-257

19. Smellie WS (2007) Spurious hyperkalaemia. BMJ 334:693-695

20. Asirvatham JR, Moses V, Bjornson L (2013) Errors in potassium measurement: a laboratory perspective for the clinician. N Am J Med Sci 5:255-259

21. You JS, Park YS, Chung HS et al (2014) Evaluating the utility of rapid point-of-care potassium testing for the early identification of hyperkalemia in patients with chronic kidney disease in the emergency department. Yonsei Med J 55:1348-1353

22. Association UR (2014) Teatment of Acute Hyperkalaemia in Adults. Clinical Practice Guidelines. London

23. Ahmed J, Weisberg LS (2001) Hyperkalemia in dialysis patients. Semin Dial 14:348-356
24. Surawicz B, Chlebus H, Mazzoleni A (1967) Hemodynamic and electrocardiographic effects of hyperpotassemia. Differences in response to slow and rapid increases in concentration of plasma K. Am Heart J 73:647-664

25. An JN, Lee JP, Jeon HJ et al (2012) Severe hyperkalemia requiring hospitalization: predictors of mortality. Crit Care 16:R225

26. Elliott MJ, Ronksley PE, Clase CM, Ahmed SB, Hemmelgarn BR (2010) Management of patients with acute hyperkalemia. CMAJ 182:1631-1635

27. Apel J, Reutrakul S, Baldwin D (2014) Hypoglycemia in the treatment of hyperkalemia with insulin in patients with end-stage renal disease. Clin Kidney J 7:248-250

28. Alfonzo AV, Isles C, Geddes C, Deighan C (2006) Potassium disorders-clinical spectrum and emergency management. Resuscitation 70:10 25

29. El-Sherif N, Turitto G (2011) Electrolyte disorders and arrhythmogenesis. Cardiol J 18:233-245

30. Paice BJ, Paterson KR, Onyanga-Omara F, Donnelly T, Gray JM, Lawson DH (1986) Record linkage study of hypokalaemia in hospitalized patients. Postgrad Med J 62:187-191

31. Kjeldsen K (2010) Hypokalemia and sudden cardiac death. Exp Clin Cardiol 15:e96-e99

32. Cohn JN, Kowey PR, Whelton PK, Prisant LM (2000) New guidelines for potassium replacement in clinical practice: a contemporary review by the National Council on Potassium in Clinical Practice. Arch Intern Med 160:2429-2436

33. Brown DJ, Brugger H, Boyd J, Paal P (2012) Accidental hypothermia. N Engl J Med 367:19301938

34. Pasquier M, Zurron N, Weith B et al (2014) Deep accidental hypothermia with core temperature below 24 degrees c presenting with vital signs. High Alt Med Biol 15:58-63

35. Walpoth BH, Galdikas J, Leupi F, Muehlemann W, Schlaepfer P, Althaus U (1994) Assessment of hypothermia with a new "tympanic" thermometer. J Clin Monit 10:91-96

36. Strapazzon G, Procter E, Paal P, Brugger H (2014) Pre-hospital core temperature measurement in accidental and therapeutic hypothermia. High Alt Med Biol 15:104-111

37. Brugger $\mathrm{H}$, Oberhammer $\mathrm{R}$, Adler-Kastner $\mathrm{L}$, Beikircher W (2009) The rate of cooling during

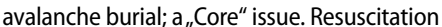
80:956-958

38. Lefrant JY, Muller L, de La Coussaye JE et al (2003) Temperature measurement in intensive care patients: comparison of urinary bladder, oesophageal, rectal, axillary, and inguinal methods versus pulmonary artery core method. Intensive Care Med 29:414-418

39. Robinson J, Charlton J, Seal R, Spady D, Joffres MR (1998) Oesophageal, rectal, axillary, tympanic and pulmonary artery temperatures during cardiac surgery. Can J Anaesth 45:317-323

40. Wood S (1991) Interactions between hypoxia and hypothermia. Ann Rev Physiol 53:71-85

41. Schneider SM (1992) Hypothermia: from recognition to rewarming. Emerg Med Rep 13:120

42. Gilbert $M$, Busund $R$, Skagseth $A$, Nilsen PA, Solbo JP (2000) Resuscitation from accidental hypothermia of 13.7 degrees $C$ with circulatory arrest. Lancet 355:375-376

43. Lexow K (1991) Severe accidental hypothermia: survival after 6 hours 30 min of cardiopulmonary resuscitation. Arctic Med Res 50(Suppl 6):112114 
44. Boue $Y$, Lavolaine J, Bouzat $P$, Matraxia $S$, Chavanon O, Payen JF (2014) Neurologic recovery from profound accidental hypothermia after 5 hours of cardiopulmonary resuscitation. Crit Care Med 42:e167-e170

45. Gordon L, Paal P, Ellerton JA, Brugger H, Peek GJ, Zafren K (2015) Delayed and intermittent CPR for severe accidental hypothermia. Resuscitation 90:46-49

46. Paal P, Milani M, Brown D, Boyd J, Ellerton $J$ (2012) Termination of cardiopulmonary resuscitation in mountain rescue. High Alt Med Biol 13:200-208

47. Danzl DF, Pozos RS, Auerbach PS et al (1987) Multicenter hypothermia survey. Ann Emerg Med 16:1042-1055

48. Putzer G, Tiefenthaler W, Mair P, Paal P (2012) Near-infrared spectroscopy during cardiopulmonary resuscitation of a hypothermic polytraumatised cardiac arrest patient. Resuscitation 83:e1-e2

49. Nolan JP, Soar J, Wenzel V, Paal P (2012) Cardiopulmonary resuscitation and management of cardiac arrest. Nat Rev Cardiol 9:499-511

50. Putzer G, Braun P, Zimmermann A et al (2013) LUCAS compared to manual cardiopulmonary resuscitation is more effective during helicopter rescue-a prospective, randomized, cross-over manikin study. Am J Emerg Med 31:384-389

51. Paal P, Beikircher W, Brugger H (2006) [Avalanche emergencies. Review of the current situation]. Anaesthesist 55:314-324

52. Stoner J, Martin G, O'Mara K, Ehlers J, Tomlanovich M (2003) Amiodarone and bretylium in the treatment of hypothermic ventricular fibrillation in a canine model. Acad Emerg Med 10:187-191

53. Krismer AC, Lindner KH, Kornberger R et al (2000) Cardiopulmonary resuscitation during severe hypothermia in pigs: does epinephrine or vasopressin increase coronary perfusion pressure? Anesth Analg 90:69-73

54. Kornberger $\mathrm{E}$, Lindner $\mathrm{KH}$, Mayr VD et al (2001) Effects of epinephrine in a pig model of hypothermic cardiac arrest and closed-chest cardiopulmonary resuscitation combined with active rewarming. Resuscitation 50:301-308

55. Mattu A, Brady WJ, Perron AD (2002) Electrocardiographic manifestations of hypothermia. Am J Emerg Med 20:314-326

56. Paal P, Strapazzon G, Braun P et al (2013) Factors affecting survival from avalanche burial $-\mathrm{a}$ randomised prospective porcine pilot study. Resuscitation 84:239-243

57. Ujhelyi MR, Sims JJ, Dubin SA, Vender J, Miller AW (2001) Defibrillation energy requirements and electrical heterogeneity during total body hypothermia. Crit Care Med 29:1006-1011

58. Zafren K, Giesbrecht GG, Danzl DF et al (2014) Wilderness Medical Society practice guidelines for the out-of-hospital evaluation and treatment of accidental hypothermia: 2014 update. Wilderness Environ Med 25:S66-S85

59. Henriksson O, Lundgren PJ, Kuklane K et al (2015) Protection against cold in prehospital care: wet clothing removal or addition of a vapor barrier. Wilderness Environ Med 26:11-20

60. Brown D, Ellerton J, Paal P, Boyd J (2015) Hypothermia Evidence, Afterdrop, and Practical Experience. Wilderness Environ Med 26(3):437439
61. Lundgren $P$, Henriksson $O$, Naredi P, Bjornstig $U$ (2011) The effect of active warming in prehospital trauma care during road and air ambulance transportation - a clinical randomized trial. Scand J Trauma Resusc Emerg Med 19:59

62. Gruber E, Beikircher W, Pizzinini R et al (2014) Non-extracorporeal rewarming at a rate of 6.8 degrees $C$ per hour in a deeply hypothermic arrested patient. Resuscitation 85:e119-e120

63. Bouchama A, Knochel JP (2002) Heat stroke. N Engl J Med 346:1978-1988

64. Wappler F (2001) Malignant hyperthermia. Eur J Anaesthesiol 18:632-652

65. Ali SZ, Taguchi A, Rosenberg H (2003) Malignant hyperthermia. Best Pract Res Clin Anaesthesiol 17:519-533

66. Empana JP, Sauval $P$, Ducimetiere $P$, Tafflet $M$, Carli P, Jouven X (2009) Increase in out-ofhospital cardiac arrest attended by the medical mobile intensive care units, but not myocardial infarction, during the 2003 heat wave in Paris, France. Crit Care Med 37:3079-3084

67. Coris EE, Ramirez AM, Van Durme DJ (2004) Heat illness in athletes: the dangerous combination of heat, humidity and exercise. Sports Med 34:9-16

68. Grogan H, Hopkins PM (2002) Heat stroke: implications for critical care and anaesthesia. $\mathrm{Br}$ Anaesth 88:700-707

69. Bouchama A, De Vol EB (2001) Acid-base alterations in heatstroke. Inten Care Med 27:680685

70. Pease S, Bouadma L, Kermarrec N, Schortgen F, Regnier B, Wolff M (2009) Early organ dysfunction course, cooling time and outcome in classic heatstroke. Inten Care Med 35:1454-1458

71. Akhtar M, Jazayeri MR, Sra J, Blanck Z Deshpande S, Dhala A (1993) Atrioventricular nodal reentry: clinical, electrophysiological, and therapeutic considerations. Circulation 88:282295

72. el-Kassimi FA, Al-Mashhadani S, Abdullah AK, Akhtar J (1986) Adult respiratory distress syndrome and disseminated intravascular coagulation complicating heat stroke. Chest 90:571-574

73. Waruiru C, Appleton R (2004) Febrile seizures: an update. Arch Dis Childhood 89:751-756

74. Berger J, Hart J, Millis M, Baker AL (2000) Fulminant hepatic failure from heat stroke requiring liver transplantation. J Clin Gastroenterol 30:429-431

75. Huerta-Alardin AL, Varon J, Marik PE (2005) Bench-to-bedside review: rhabdomyolysis - an overview for clinicians. Crit Care 9:158-169

76. Hadad E, Weinbroum AA, Ben-Abraham R (2003) Drug-induced hyperthermia and muscle rigidity: a practical approach. Eur J Emerg Med 10:149154

77. Halloran LL, Bernard DW (2004) Management of drug-induced hyperthermia. Curr Opin Pediatr 16:211-215

78. Bouchama A, Dehbi M, Chaves-Carballo E (2007) Cooling and hemodynamic management in heatstroke: practical recommendations. Crit Care 11:R54

79. Armstrong $\mathrm{LE}, \mathrm{Crago} \mathrm{AE}$, Adams $\mathrm{R}$, Roberts WO, Maresh CM (1996) Whole-body cooling of hyperthermic runners: comparison of two field therapies. Am J Emerg Med 14:355-358

80. Nolan JP, Soar J, Cariou A et al (2015) European Resuscitation Council Guidelines for Resuscitation 2015 Section 5 Post Resuscitation Care. Resuscitation
81. Horowitz BZ (1989) The golden hour in heat stroke: use of iced peritoneal lavage. Am J Emerg Med 7:616-619

82. Bernard S, Buist M, Monteiro O, Smith K (2003) Induced hypothermia using large volume, icecold intravenous fluid in comatose survivors of out-of-hospital cardiac arrest: a preliminary report. Resuscitation 56:9-13

83. Al-Senani FM, Graffagnino C, Grotta JC et al (2004) A prospective, multicenter pilot study to evaluate the feasibility and safety of using the CoolGard System and Icy catheter following cardiac arrest. Resuscitation 62:143-150

84. Schmutzhard E, Engelhardt K, Beer R et al (2002) Safety and efficacy of a novel intravascular cooling device to control body temperature in neurologic intensive care patients: a prospective pilot study. Crit Care Med 30:2481-2488

85. Behringer W, Safar P, Wu X et al (2002) Venovenous extracorporeal blood shunt cooling to induce mild hypothermia in dog experiments and review of cooling methods. Resuscitation 54:89-98

86. Hostler D, Northington WE, Callaway CW (2009) High-dose diazepam facilitates core cooling during cold saline infusion in healthy volunteers. Appl Physiol Nutr Metab 34:582-586

87. Hadad E, Cohen-Sivan Y, Heled Y, Epstein Y (2005) Clinical review: treatment of heat stroke: should dantrolene be considered? Crit Care 9:86-91

88. Channa AB, Seraj MA, Saddique AA, Kadiwal GH, Shaikh MH, Samarkandi AH (1990) Is dantrolene effective in heat stroke patients? Crit Care Med 18:290-292

89. Bouchama A, Cafege A, Devol EB, Labdi O, el-Assil K, Seraj M (1991) Ineffectiveness of dantrolene sodium in the treatment of heatstroke. Crit Care Med 19:176-180

90. Larach MG, Gronert GA, Allen GC, Brandom BW, Lehman EB (2010) Clinical presentation, treatment, and complications of malignant hyperthermia in North America from 1987 to 2006. Anesth Analg 110:498-507

91. Krause T, Gerbershagen MU, Fiege M, Weisshorn R, Wappler F (2004) Dantrolene - a review of its pharmacology, therapeutic use and new developments. Anaesthesia 59:364-373

92. Hall AP, Henry JA (2006) Acute toxic effects of „Ecstasy' (MDMA) and related compounds: overview of pathophysiology and clinical management. Br J Anaesth 96:678-685

93. Eshel G, Safar P, Sassano J, Stezoski W (1990) Hyperthermia-induced cardiac arrest in dogs and monkeys. Resuscitation 20:129-143

94. Eshel G, Safar P, Radovsky A, Stezoski SW (1997) Hyperthermia-induced cardiac arrest in monkeys: limited efficacy of standard CPR. Aviat Space Environ Med 68:415-420

95. Zeiner A, Holzer M, Sterz F et al (2001) Hyperthermia after cardiac arrest is associated with an unfavorable neurologic outcome. Arch Intern Med 161:2007-2012

96. Muraro A, Roberts G, Worm M et al (2014) Anaphylaxis: guidelines from the European Academy of Allergy and Clinical Immunology. Allergy 69:1026-1045

97. Kleber C, Giesecke MT, Lindner T, Haas NP, Buschmann CT (2014) Requirement for a structured algorithm in cardiac arrest following major trauma: epidemiology, management errors, and preventability of traumatic deaths in Berlin. Resuscitation 85:405-410 
98. Brenner ML, Moore LJ, DuBose JJ et al (2013) A clinical series of resuscitative endovascular balloon occlusion of the aorta for hemorrhage control and resuscitation. J Trauma Acute Care Surg 75:506-511

99. Simons FE, Ardusso LR, Bilo MB et al (2014) International consensus on (ICON) anaphylaxis. World Allergy Organ J 7:9

100. Johansson SG, Bieber T, Dahl R et al (2004) Revised nomenclature for allergy for global use: report of the Nomenclature Review Committee of the World Allergy Organization, October 2003. J Allergy Clin Immunol 113:832-836

101. Soar J, Pumphrey R, Cant A et al (2008) Emergency treatment of anaphylactic reactions guidelines for healthcare providers. Resuscitation 77:157-169

102. Soar J (2009) Emergency treatment of anaphylaxis in adults: concise guidance. Clin Med 9:181-185

103. Panesar SS, Javad S, de Silva D et al (2013) The epidemiology of anaphylaxis in Europe: a systematic review. Allergy 68:1353-1361

104. Muraro A, Roberts G, Clark A et al (2007) The management of anaphylaxis in childhood: position paper of the European academy of allergology and clinical immunology. Allergy 62:857-871

105. Harper NJ, Dixon T, Dugue P et al (2009) Suspected anaphylactic reactions associated with anaesthesia. Anaesthesia 64:199-211

106. Turner PJ, Gowland MH, Sharma V et al (2015) Increase in anaphylaxis-related hospitalizations but no increase in fatalities: an analysis of United Kingdom national anaphylaxis data, 1992-2012. J Allergy Clin Immunol 135:956-963e1

107. Worm M, Moneret-Vautrin A, Scherer K et al (2014) First European data from the network of severe allergic reactions (NORA). Allergy 69:1397-1404

108. Gibbison B, Sheikh A, McShane P, Haddow C, Soar $J$ (2012) Anaphylaxis admissions to UK critical care units between 2005 and 2009. Anaesthesia 67:833-839

109. Pumphrey RS (2004) Fatal anaphylaxis in the UK, 1992-2001. Novartis Found Symp 257:116-128 (discussion 28-32, 57-60, 276-185)

110. Gonzalez-Perez A, Aponte Z, Vidaurre CF, Rodriguez LA (2010) Anaphylaxis epidemiology in patients with and patients without asthma: a United Kingdom database review. J Allergy Clin Immunol 125:1098-1104e1

111. Pumphrey RS (2000) Lessons for management of anaphylaxis from a study of fatal reactions. Clin Exp Allergy 30:1144-1150

112. Sampson HA, Munoz-Furlong A, Campbell RL et al (2006) Second symposium on the definition and management of anaphylaxis: summary report-Second National Institute of Allergy and Infectious Disease/Food Allergy and Anaphylaxis Network symposium. J Allergy Clin Immunol 117:391-397

113. Dhami S, Panesar SS, Roberts G et al (2014) Management of anaphylaxis: a systematic review. Allergy 69:168-175

114. Pumphrey RSH (2003) Fatal posture in anaphylactic shock. J Allergy Clin Immunol 112:451-452

115. Visscher PK, Vetter RS, Camazine S (1996) Removing bee stings. Lancet 348:301-302

116. Simpson CR, Sheikh A (2010) Adrenaline is first line treatment for the emergency treatment of anaphylaxis. Resuscitation 81:641-642
117. Kemp SF, Lockey RF, Simons FE (2008) Epinephrine: the drug of choice for anaphylaxis. A statement of the World Allergy Organization. Allergy 63:1061-1070

118. Sheikh A, Shehata YA, Brown SG, Simons FE (2008) Adrenaline (epinephrine) for the treatment of anaphylaxis with and without shock. Cochrane Database Syst Rev 4:CD006312

119. Bautista E, Simons FE, Simons KJ et al (2002) Epinephrine fails to hasten hemodynamic recovery in fully developed canine anaphylactic shock. Int Arch Allergy Immunol 128:151-164

120. Song TT, Nelson MR, Chang JH, Engler RJ, Chowdhury BA (2005) Adequacy of the epinephrine autoinjector needle length in delivering epinephrine to the intramuscular tissues. Ann Allergy Asthma Immunol 94:539542

121. Simons FE, Gu X, Simons KJ (2001) Epinephrine absorption in adults: intramuscular versus subcutaneous injection. J Allergy Clin Immunol 108:871-873

122. Simons FE, Roberts JR, Gu X, Simons KJ (1998) Epinephrine absorption in children with a history of anaphylaxis. J Allergy Clin Immunol 101:33-37

123. Simons FE, Gu X, Johnston LM, Simons KJ (2000) Can epinephrine inhalations be substituted for epinephrine injection in children at risk for systemic anaphylaxis? Pediatrics 106:1040-1044

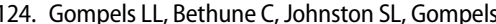
MM (2002) Proposed use of adrenaline (epinephrine) in anaphylaxis and related conditions: a study of senior house officers starting accident and emergency posts. Postgrad Med J 78:416-418

125. Brown SG, Blackman KE, Stenlake V, Heddle RJ (2004) Insect sting anaphylaxis; prospective evaluation of treatment with intravenous adrenaline and volume resuscitation. Emerg Med J 21:149-154

126. Brown SG (2005) Cardiovascular aspects of anaphylaxis: implications for treatment and diagnosis. Curr Opin Allergy Clin Immunol 5:359364

127. O'Driscoll BR, Howard LS, Davison AG (2008) BTS guideline for emergency oxygen use in adult patients. Thorax 63(Suppl 6):vi1-vi68

128. Sheikh A, Ten Broek V, Brown SG, Simons FE (2007) $\mathrm{H} 1$-antihistamines for the treatment of anaphylaxis: cochrane systematic review. Allergy 62:830-837

129. Choo KJ, Simons FE, Sheikh A (2010) Glucocorticoids for the treatment of anaphylaxis. Cochrane Database Syst Rev 3:CD007596

130. Green R, Ball A (2005) Alpha-agonists for the treatment of anaphylactic shock. Anaesthesia 60:621-622

131. Kluger MT (2001) The Bispectral Index during an anaphylactic circulatory arrest. Anaesth Inten Care 29:544-547

132. McBrien ME, Breslin DS, Atkinson S, Johnston JR (2001) Use of methoxamine in the resuscitation of epinephrine-resistant electromechanical dissociation. Anaesthesia 56:1085-1089

133. Rocq N, Favier JC, Plancade D, Steiner T, Mertes PM (2007) Successful use of terlipressin in postcardiac arrest resuscitation after an epinephrineresistant anaphylactic shock to suxamethonium. Anesthesiology 107:166-167

134. Kill C, Wranze E, Wulf H (2004) Successful treatment of severe anaphylactic shock with vasopressin. Two case reports. Int Arch Allergy Immunol 134:260-261
135. Dewachter $P$, Raeth-Fries I, Jouan-Hureaux V et al (2007) A comparison of epinephrine only, arginine vasopressin only, and epinephrine followed by arginine vasopressin on the survival rate in a rat model of anaphylactic shock. Anesthesiology 106:977-983

136. Higgins DJ, Gayatri $P$ (1999) Methoxamine in the management of severe anaphylaxis. Anaesthesia 54:1126

137. Heytman M, Rainbird A (2004) Use of alphaagonists for management of anaphylaxis occurring under anaesthesia: case studies and review. Anaesthesia 59:1210-1215

138. Schummer W, Schummer C, Wippermann J, Fuchs J (2004) Anaphylactic shock: is vasopressin the drug of choice? Anesthesiology 101:10251027

139. Di Chiara LS, Ricci Z et al (2008) Role of vasopressin in the treatment of anaphylactic shock in a child undergoing surgery for congenital heart disease: a case report. J Med Case Reports 2:36

140. Meng L, Williams EL (2008) Case report: treatment of rocuronium-induced anaphylactic shock with vasopressin. Can J Anaesth 55:437-440

141. Schummer C, Wirsing M, Schummer W (2008) The pivotal role of vasopressin in refractory anaphylactic shock. Anesth Analg 107:620-624

142. Hiruta A, Mitsuhata $H$, Hiruta M et al (2005) Vasopressin may be useful in the treatment of systemic anaphylaxis in rabbits. Shock 24:264269

143. Thomas M, Crawford I (2005) Best evidence topic report. Glucagon infusion in refractory anaphylactic shock in patients on beta-blockers. Emerg Med J 22:272-273

144. Allen SJ, Gallagher A, Paxton LD (2000) Anaphylaxis to rocuronium. Anaesthesia 55:1223-1224

145. Lafforgue E, Sleth JC, Pluskwa F, Saizy C (2005) [Successful extracorporeal resuscitation of a probable perioperative anaphylactic shock due to atracurium]. Ann Fr Anesth Reanim 24:551555

146. Vatsgar TT, Ingebrigtsen O, Fjose LO, Wikstrom B, Nilsen JE, Wik L (2006) Cardiac arrest and resuscitation with an automatic mechanical chest compression device (LUCAS) due to anaphylaxis of a woman receiving caesarean section because of pre-eclampsia. Resuscitation 68:155-159

147. Schwartz LB (2006) Diagnostic value of tryptase in anaphylaxis and mastocytosis. Immunol Allergy Clin North Am 26:451-463

148. Brown SG, Blackman KE, Heddle RJ (2004) Can serum mast cell tryptase help diagnose anaphylaxis? Emerg Med Australas 16:120-124

149. Tole JW, Lieberman P (2007) Biphasic anaphylaxis: review of incidence, clinical predictors, and observation recommendations. Immunol Allergy Clin North Am 27:309-326, viii

150. Simons FE, Lieberman PL, Read EJ Jr, Edwards ES (2009) Hazards of unintentional injection of epinephrine from autoinjectors: a systematic review. Ann Allergy Asthma Immunol 102:282287

151. Campbell RL, Luke A, Weaver AL et al (2008) Prescriptions for self-injectable epinephrine and follow-up referral in emergency department patients presenting with anaphylaxis. Ann Allergy Asthma Immunol 101:631-636

152. Kelso JM (2006) Adose of epinephrine for anaphylaxis: how often needed and how to carry. J Allergy Clin Immunol 117:464-465 
153. Choo K, Sheikh A (2007) Action plans for the long-term management of anaphylaxis: systematic review of effectiveness. Clin Exp Allergy 37:1090-1094

154. Zwingmann J, Mehlhorn AT, Hammer T, Bayer J, Sudkamp NP, Strohm PC (2012) Survival and neurologic outcome after traumatic out-ofhospital cardiopulmonary arrest in a pediatric and adult population: a systematic review. Crit Care 16:R117

155. Leis CC, Hernandez CC, Blanco MJ, Paterna PC, Hernandez Rde E, Torres EC (2013) Traumatic cardiac arrest: should advanced life support be initiated? J Trauma Acute Care Surg 74:634-638

156. Cureton EL, Yeung LY, Kwan RO et al (2012) The heart of the matter: utility of ultrasound of cardiac activity during traumatic arrest. J Trauma Acute Care Surg 73:102-110

157. Engdahl J, Herlitz J (2005) Localization of out-ofhospital cardiac arrest in Goteborg 1994-2002 and implications for public access defibrillation. Resuscitation 64:171-175

158. Ong ME, Tan EH, Yan X et al (2008) An observational study describing the geographictime distribution of cardiac arrests in Singapore: what is the utility of geographic information systems for planning public access defibrillation? (PADS Phase I). Resuscitation 76:388-396

159. Stratton SJ, Brickett K, Crammer T (1998) Prehospital pulseless, unconscious penetrating trauma victims: field assessments associated with survival. J Trauma 45:96-100

160. Cera SM, Mostafa G, Sing RF, Sarafin JL, Matthews BD, Heniford BT (2003) Physiologic predictors of survival in post-traumatic arrest. Am Surg 69:140-144

161. Powell DW, Moore EE, Cothren CC et al (2004) Is emergency department resuscitative thoracotomy futile care for the critically injured patient requiring prehospital cardiopulmonary resuscitation? J Am Coll Surg 199:211-215

162. Esposito TJ, Jurkovich GJ, Rice CL, Maier RV, Copass MK, Ashbaugh DG 1991 Reappraisal of emergency room thoracotomy in a changing environment. J Trauma 31:881-885 (discussion 5-7)

163. Martin SK, Shatney CH, Sherck JP et al (2002) Blunt trauma patients with prehospital pulseless electrical activity (PEA): poor ending assured. J trauma 53:876-880 (discussion 80-81)

164. Millin MG, Galvagno SM, Khandker SR et al (2013) Withholding and termination of resuscitation of adult cardiopulmonary arrest secondary to trauma: resource document to the joint NAEMSPACSCOT position statements. J Trauma Acute Care Surg 75:459-467

165. Lockey DJ, Lyon RM, Davies GE (2013) Development of a simple algorithm to guide the effective management of traumatic cardiac arrest. Resuscitation 84:738-742

166. Sherren PB, Reid C, Habig K, Burns BJ (2013) Algorithm for the resuscitation of traumatic cardiac arrest patients in a physician-staffed helicopter emergency medical service. Crit Care 17:308

167. Smith JE, Rickard A, Wise D (2015) Traumatic cardiac arrest. J R Soc Med 108:11-16

168. Soar J, Nolan JP, Bottiger BW et al (2015) European Resuscitation Council Guidelines for Resuscitation Section 3. Adult Advanced Life Support. Resuscitation 95:99-146
169. Luna GK, Pavlin EG, Kirkman T, Copass MK, Rice $\mathrm{CL}$ (1989) Hemodynamic effects of external cardiac massage in trauma shock. J trauma 29:1430-1433

170. Willis CD, Cameron PA, Bernard SA, Fitzgerald $M$ (2006) Cardiopulmonary resuscitation after traumatic cardiac arrest is not always futile. Injury 37:448-454

171. Lockey D, Crewdson K, Davies G (2006) Traumatic cardiac arrest: who are the survivors? Ann Emerg Med 48:240-244

172. Crewdson K, Lockey D, Davies G (2007) Outcome from paediatric cardiac arrest associated with trauma. Resuscitation 75:29-34

173. Spahn DR, Bouillon B, Cerny V et al (2013) Management of bleeding and coagulopathy following major trauma: an updated European guideline. Crit Care 17:R76

174. Kwan I, Bunn F, Chinnock P, Roberts I (2014) Timing and volume of fluid administration for patients with bleeding. Cochrane Database Syst Rev 3:CD002245

175. Bickell WH, Wall MJ Jr, Pepe PE et al (1994) Immediate versus delayed fluid resuscitation for hypotensive patients with penetrating torso injuries. N Engl J Med 331:1105-1109

176. Harris T, Thomas GO, Brohi K (2012) Early fluid resuscitation in severe trauma. BMJ 345:e5752

177. Jansen JO, Thomas R, Loudon MA, Brooks A (2009) Damage control resuscitation for patients with major trauma. BMJ 338:b1778

178. Holcomb JB, Tilley BC, Baraniuk S et al (2015) Transfusion of plasma, platelets, and red blood cells in a 1:1:1 vs a 1:1:2 ratio and mortality in patients with severe trauma: the PROPPR randomized clinical trial. JAMA 313:471-482

179. Bodnar D, Rashford S, Hurn C et al (2013) Characteristics and outcomes of patients administered blood in the prehospital environment by a road based trauma response team. Emerg Med J [Epub ahead of print]

180. Lockey DJ, Weaver AE, Davies GE (2013) Practica translation of hemorrhage control techniques to the civilian trauma scene. Transfusion 53(Suppl 1):17S-22S

181. Holcomb JB, Jenkins D, Rhee P et al (2007) Damage control resuscitation: directly addressing the early coagulopathy of trauma. J Trauma 62:307-310

182. collaborators $\mathrm{C}-$, Roberts $\mathrm{I}$, Shakur $\mathrm{H}$ et al (2011) The importance of early treatment with tranexamic acid in bleeding trauma patients: an exploratory analysis of the CRASH-2 randomised controlled trial. Lancet 377:1096-1101, 101 e1-2

183. Cobas MA, De la Pena MA, Manning R, Candiotti K, Varon AJ (2009) Prehospital intubations and mortality: a level 1 trauma center perspective. Anesth Analg 109:489-493

184. Lockey DJ, Healey B, Crewdson K, Chalk G, Weaver AE, Davies GE (2015) Advanced airway management is necessary in prehospital trauma patients. Br J Anaesth 114:657-662

185. Pepe PE, Roppolo LP, Fowler RL (2005) The detrimental effects of ventilation during lowblood-flow states. Curr Opin Crit Care 11:212218

186. Escott ME, Gleisberg GR, Kimmel K, Karrer A, Cosper J, Monroe BJ (2014) Simple thoracostomy. Moving beyong needle decompression in traumatic cardiac arrest. JEMS 39:26-32

187. Deakin CD, Davies G, Wilson A (1995) Simple thoracostomy avoids chest drain insertion in prehospital trauma. J Trauma 39:373-374
188. Flaris AN, Simms ER, Prat N, Reynard F, Caillot JL, Voiglio EJ (2015) Clamshell incision versus left anterolateral thoracotomy. Which one is faster when performing a resuscitative thoracotomy? The tortoise and the hare revisited. World J Surg 39:1306-1311

189. Wise D, Davies G, Coats T, Lockey D, Hyde J, Good A (2005) Emergency thoracotomy:, how to do it“. Emerg Med J 22:22-24

190. Rhee PM, Acosta J, Bridgeman A, Wang D, Jordan $M$, Rich N (2000) Survival after emergency department thoracotomy: review of published data from the past 25 years. J Am Coll Surg 190:288-298

191. Burlew CC, Moore EE, Moore FA et al (2012) Western Trauma Association critical decisions in trauma: resuscitative thoracotomy. JTrauma Acute Care Surg 73:1359-1363

192. Matsumoto H, Mashiko K, Hara Y et al (2009) Role of resuscitative emergency field thoracotomy in the Japanese helicopter emergency medical service system. Resuscitation 80:1270-1274

193. Seamon MJ, Chovanes J, Fox $\mathrm{N}$ et al (2012) The use of emergency department thoracotomy for traumatic cardiopulmonary arrest. Injury 43:1355-1361

194. Gao JM, Gao YH, Wei GB et al (2004) Penetrating cardiac wounds: principles for surgical management. World J Surg 28:1025-1029

195. Manz E, Nofz L, Norman A, Davies GE (2013) Incidence of clotted heamopericardium in traumatic cardiac arrest in 152 thoracotomy patients. Scan J Trauma Resusc Emerg Med 22:P20

196. Ferrada P, Wolfe L, Anand RJ et al (2014) Use of limited transthoracic echocardiography in patients with traumatic cardiac arrest decreases the rate of nontherapeutic thoracotomy and hospital costs. J Ultrasound Med 33:1829-1832

197. Walcher F, Kortum S, Kirschning T, Weihgold $\mathrm{N}$, Marzi I (2002) [Optimized management of polytraumatized patients by prehospital ultrasound]. Unfallchirurg 105:986-994

198. Huber-Wagner S, Lefering R, Qvick LM et al (2009) Effect of whole-body CT during trauma resuscitation on survival: a retrospective, multicentre study. Lancet 373:1455-1461

199. Barton ED (1999) Tension pneumothorax. Curr Opin Pulm Med 5:269-274

200. Roberts DJ, Leigh-Smith S, Faris PD et al (2015) Clinical presentation of patients with tension pneumothorax: a systematic review. Ann Surg [Epub ahead of print]

201. Leigh-Smith S, Harris T (2005) Tension pneumothorax-time for a re-think? Emerg Med J 22:8-16

202. Chen KY, Jerng JS, Liao WY et al (2002) Pneumothorax in the ICU: patient outcomes and prognostic factors. Chest 122:678-683

203. Warner KJ, Copass MK, Bulger EM (2008) Paramedic use of needle thoracostomy in the prehospital environment. Prehosp Emerg Care 12:162-168

204. Mistry N, Bleetman A, Roberts KJ (2009) Chest decompression during the resuscitation of patients in prehospital traumatic cardiac arrest. Emerg Med J 26:738-740

205. Clemency BM, Tanski CT, Rosenberg M, May PR, Consiglio JD, Lindstrom HA (2015) Sufficient catheter length for pneumothorax needle decompression: a meta-analysis. Prehosp Disaster Med 30:249-253 
206. Holcomb JB, McManus JG, Kerr ST, Pusateri AE (2009) Needle versus tube thoracostomy in a swine model of traumatic tension hemopneumothorax. Prehosp Disaster Med 13:18-27

207. Massarutti D, Trillo G, Berlot G et al (2006) Simple thoracostomy in prehospital trauma management is safe and effective: a 2-year experience by helicopter emergency medical crews. Emerg Med 13:276-280

208. Konstantinides SV, Torbicki A, Agnelli G et al (2014) ESC guidelines on the diagnosis and management of acute pulmonary embolism. Eur Heart J 35:3033-3069, 69a-69k

209. Heradstveit BE, Sunde K, Sunde GA, WentzelLarsen T, Heltne JK (2012) Factors complicating interpretation of capnography during advanced life support in cardiac arrest-A clinical retrospective study in 575 patients. Resuscitation 83:813-818

210. Kurkciyan I, Meron G, Behringer W et al (1998) Accuracy and impact of presumed cause in patients with cardiac arrest. Circulation 98:766771

211. Kurkciyan I, Meron G, Sterz F et al (2000) Pulmonary embolism as a cause of cardiac arrest: presentation and outcome. Arch Intern Med 160:1529-1535

212. Pokorna M, Necas E, Skripsky R, Kratochvil J, Andrlik M, Franek O (2011) How accurately can the aetiology of cardiac arrest be established in an out-of-hospital setting? Analysis by "concordance in diagnosis crosscheck tables". Resuscitation 82:391-397

213. Wallmuller C, Meron G, Kurkciyan I, Schober A, Stratil P, Sterz F (2012) Causes of in-hospital cardiac arrest and influence on outcome. Resuscitation 83:1206-1211

214. Bergum D, Nordseth T, Mjolstad OC, Skogvoll E, Haugen BO (2015) Causes of in-hospital cardiac arrest - incidences and rate of recognition. Resuscitation 87:63-68

215. Bottiger BW, Arntz HR, Chamberlain DA et al (2008) Thrombolysis during resuscitation for out-of-hospital cardiac arrest. N Engl J Med 359:2651-2662

216. Silfvast T (1991) Cause of death in unsuccessful prehospital resuscitation. J Intern Med 229:331335

217. Heit JA, Silverstein MD, Mohr DN, Petterson TM O'Fallon WM, Melton LJ 3rd (2000) Risk factors for deep vein thrombosis and pulmonary embolism: a population-based case-control study. Arch Intern Med 160:809-815

218. White RH (2003) The epidemiology of venous thromboembolism. Circulation 107:14-18

219. Geibel A, Zehender M, Kasper W, Olschewski M, Klima C, Konstantinides SV (2005) Prognostic value of the ECG on admission in patients with acute major pulmonary embolism. European Respir J 25:843-848

220. Torbicki A, Pruszczyk P (2001) The role of echocardiography in suspected and established PE. Semin Vasc Med 1:165-174

221. MacCarthy P, Worrall A, McCarthy G, Davies J (2002) The use of transthoracic echocardiography to guide thrombolytic therapy during cardiac arrest due to massive pulmonary embolism. Emerg Med J 19:178-179

222. Legome E, Pancu D (2004) Future applications for emergency ultrasound. Emerg Med Clin North Am 22:817-827
223. Roy PM, Colombet I, Durieux P, Chatellier G, Sors H, Meyer G (2005) Systematic review and meta-analysis of strategies for the diagnosis of suspected pulmonary embolism. BMJ 331:259

224. Bova C, Greco F, Misuraca G et al (2003) Diagnostic utility of echocardiography in patients with suspected pulmonary embolism. Am J Emerg Med 21:180-183

225. Li X, Fu QL, Jing XL et al (2006) A meta-analysis of cardiopulmonary resuscitation with and without the administration of thrombolytic agents. Resuscitation 70:31-36

226. Janata K, Holzer M, Kurkciyan I et al (2003) Major bleeding complications in cardiopulmonary resuscitation: the place of thrombolytic therapy in cardiac arrest due to massive pulmonary embolism. Resuscitation 57:49-55

227. Böttiger BW, Martin E (2001) Thrombolytic therapy during cardiopulmonary resuscitation and the role of coagulation activation after cardiac arrest. Curr Opin Crit Care 7:176-183

228. Fatovich DM, Dobb GJ, Clugston RA (2004) A pilot randomised trial of thrombolysis in cardiac arrest (The TICA trial). Resuscitation 61:309-313

229. Konstantinov IE, Saxena P, Koniuszko MD, Alvarez J, Newman MA (2007) Acute massive pulmonary embolism with cardiopulmonary resuscitation: management and results. Tex Heart Inst J 34:4145 (discussion 5-6)

230. Zahorec R (2002) Rescue systemic thrombolysis during cardiopulmonary resuscitation. Bratisl Lek Listy 103:266-269

231. Lederer W, Lichtenberger $C$, Pechlaner $C$, Kroesen G, Baubin M (2001) Recombinant tissue plasminogen activator during cardiopulmonary resuscitation in 108 patients with out-of-hospital cardiac arrest. Resuscitation 50:71-76

232. Spöhr F, Böttiger BW (2003) Safety of thrombolysis during cardiopulmonary resuscitation. Drug Saf 26:367-379

233. Wu JP, Gu DY, Wang S, Zhang ZJ, Zhou JC, Zhang RF (2014) Good neurological recovery after rescue thrombolysis of presumed pulmonary embolism despite prior 100 minutes CPR. J Thorac Dis 6:E289-E293

234. Maj G, Melisurgo G, De Bonis M, Pappalardo $F$ (2014) ECLS management in pulmonary embolism with cardiac arrest: which strategy is better? Resuscitation 85:e175-e176

235. Swol J, Buchwald D, Strauch J, Schildhauer TA (2015) Extracorporeal life support (ECLS) for cardiopulmonary resuscitation (CPR) with pulmonary embolism in surgical patients - a case series. Perfusion

236. Doerge HC, Schoendube FA, Loeser H, Walter M, Messmer BJ (1996) Pulmonary embolectomy: review of a 15-year experience and role in the age of thrombolytic therapy. Eur J Cardiothorac Surg 10:952-957

237. Fava M, Loyola S, Bertoni H, Dougnac A (2005) Massive pulmonary embolism: percutaneous mechanical thrombectomy during cardiopulmonary resuscitation. J Vasc Interv Radiol 16:119-123

238. Hashiba K, Okuda J, Maejima N et al (2012) Percutaneous cardiopulmonary support in pulmonary embolism with cardiac arrest. Resuscitation 83:183-187

239. Miller AC, Rosati SF, Suffredini AF, Schrump DS (2014) A systematic review and pooled analysis of CPR-associated cardiovascular and thoracic injuries. Resuscitation 85:724-731
240. Smekal D, Lindgren E, Sandler H, Johansson J, Rubertsson S (2014) CPR-related injuries after manual or mechanical chest compressions with the LUCAS device: a multicentre study of victims after unsuccessful resuscitation. Resuscitation 85:1708-1712

241. Truhlar A, Hejna P, Zatopkova L, Skulec R, Cerny V (2012) Concerns about safety of the AutoPulse use in treatment of pulmonary embolism. Resuscitation 83:e133-e134 (discussion e5)

242. Nikolaou NI, Arntz HR, Bellou A, Beygui F, Bossaert LL, Cariou A (2015) European Resuscitation Council Guidelines for Resuscitation 2015 Section 5. Initial Management of Acute Coronary Syndromes Resuscitation

243. Bossaert L, Perkins GD, Askitopoulou H et al (2015) European Resuscitation Council Guidelines for Resuscitation Section 11. The ethics of resuscitation and end-of-life decisions. Resuscitation 95:301-310

244. Lamhaut L, Jouffroy R, Soldan M et al (2013) Safety and feasibility of prehospital extra corporeal life support implementation by nonsurgeons for out-of-hospital refractory cardiac arrest. Resuscitation 84:1525-1529

245. Maekawa K, Tanno K, Hase M, Mori K, Asai Y (2013) Extracorporeal cardiopulmonary resuscitation for patients with out-of-hospital cardiac arrest of cardiac origin: a propensitymatched study and predictor analysis*. Crit Care Med 41:1186-1196

246. Sakamoto T, Morimura N, Nagao Ket al (2014) Extracorporeal cardiopulmonary resuscitation versus conventional cardiopulmonary resuscitation in adults with out-of-hospital cardiac arrest: a prospective observational study. Resuscitation 85:762-768

247. Wagner H, Terkelsen CJ, Friberg H et al (2010) Cardiac arrest in the catheterisation laboratory: a 5-year experience of using mechanical chest compressions to facilitate $\mathrm{PCl}$ during prolonged resuscitation efforts. Resuscitation 81:383-387

248. Forti A, Zilio G, Zanatta P et al (2014) Full recovery after prolonged cardiac arrest and resuscitation with mechanical chest compression device during helicopter transportation and percutaneous coronary intervention. J Emerg Med 47:632-634

249. Stub D, Bernard S, Pellegrino V et al (2015) Refractory cardiac arrest treated with mechanical CPR, hypothermia, ECMO and early reperfusion (the CHEER trial). Resuscitation 86:88-94

250. Belohlavek J, Kucera K, Jarkovsky J et al (2012) Hyperinvasive approach to out-of hospital cardiac arrest using mechanical chest compression device, prehospital intraarrest cooling, extracorporeal life support and early invasive assessment compared to standard of care. A randomized parallel groups comparative study proposal. „Prague OHCA study“. J Transl Med 10:163

251. Stub D, Nehme Z, Bernard S, Lijovic M, Kaye DM, Smith K (2014) Exploring which patients without return of spontaneous circulation following ventricular fibrillation out-of-hospital cardiac arrest should be transported to hospital? Resuscitation 85:326-331

252. Mowry JB, Spyker DA, Cantilena LR Jr, McMillan N, Ford M (2014) 2013 Annual Report of the American Association of Poison Control Centers' National Poison Data System (NPDS): 31st Annual Report. Clin Toxicol (Phila) 52:1032-1283 
253. Zimmerman JL (2003) Poisonings and overdoses in the intensive care unit: general and specific management issues. Crit Care Med 31:27942801

254. Park JH, Shin SD, Song KJ, Park CB, Ro YS, Kwak YH (2012) Epidemiology and outcomes of poisoning-induced out-of-hospital cardiac arrest. Resuscitation 83:51-57

255. Gunja N, Graudins A (2011) Management of cardiac arrest following poisoning. Emerg Med Australas 23:16-22

256. Yanagawa Y, Sakamoto T, Okada Y (2007) Recovery from a psychotropic drug overdose tends to depend on the time from ingestion to arrival, the Glasgow Coma Scale, and a sign of circulatory insufficiency on arrival. Am J Emerg Med 25:757-761

257. Thompson TM, Theobald J, Lu J, Erickson TB (2014) The general approach to the poisoned patient. Dis Mon 60:509-524

258. Engebretsen KM, Kaczmarek KM, Morgan J, Holger JS (2011) High-dose insulin therapy in beta-blocker and calcium channel-blocker poisoning. Clin Toxicol (Phila) 49:277-283

259. Cave G, Harvey MG (2014) Should we consider the infusion of lipid emulsion in the resuscitation of poisoned patients? Crit Care 18:457

260. Ozcan MS, Weinberg G (2014) Intravenous lipid emulsion for the treatment of drug toxicity. J Intensive Care Med 29:59-70

261. Agarwala R, Ahmed SZ, Wiegand TJ (2014) Prolonged use of intravenous lipid emulsion in a severe tricyclic antidepressant overdose. J Med Toxicol 10:210-214

262. Kundu R, Almasri H, Moza A, Ghose A, Assaly $R$ (2013) Intravenous lipid emulsion in wide complex arrhythmia with alternating bundle branch block pattern from cocaine overdose. Kardiol Pol 71:1073-1075

263. de Lange DW, Sikma MA, Meulenbelt J (2013) Extracorporeal membrane oxygenation in the treatment of poisoned patients. Clin Toxicol (Phila) 51:385-393

264. Masson R, Colas V, Parienti JJ et al (2012) A comparison of survival with and without extracorporeal life support treatment for severe poisoning due to drug intoxication. Resuscitation 83:1413-1417

265. Proudfoot AT, Krenzelok EP, Vale JA (2004) Position Paper on urine alkalinization. J Toxicol Clin Toxicol 42:1-26

266. Greene S, Harris C, Singer J (2008) Gastrointestinal decontamination of the poisoned patient. Pediatric emergency care 24:176-186 (quiz 87-89)

267. Benson BE, Hoppu K, Troutman WG et al (2013) Position paper update: gastric lavage for gastrointestinal decontamination. Clin Toxicol (Phila) 51:140-146

268. Vale JA, Kulig K (2004) Position paper: gastric lavage. J Toxicol Clin Toxicol 42:933-943

269. Chyka PA, Seger D, Krenzelok EP, Vale JA (2005) Position paper: single-dose activated charcoal. Clin Toxicol (Phila) 43:61-87

270. Thanacoody R, Caravati EM, Troutman B et al (2015) Position paper update: whole bowel irrigation for gastrointestinal decontamination of overdose patients. Clin Toxicol (Phila) 53:5-12

271. Krenzelok EP (2005) Ipecac syrup-induced emesis...no evidence of benefit. Clin Toxicol (Phila) 43:11-12
272. (1999) Position statement and practice guidelines on the use of multi-dose activated charcoal in the treatment of acute poisoning American Academy of Clinical Toxicology; European Association of Poisons Centres and Clinical Toxicologists. J Toxicol Clin Toxicol 37:731-751

273. Hojer J, Troutman WG, Hoppu K et al (2013) Position paper update: ipecac syrup for gastrointestinal decontamination. Clin Toxicol (Phila) 51:134-139

274. Skinner CG, Chang AS, Matthews AS, Reedy SJ, Morgan BW (2012) Randomized controlled study on the use of multiple-dose activated charcoal in patients with supratherapeutic phenytoin levels. Clin Toxicol (Phila) 50:764-769

275. Brahmi N, Kouraichi N, Thabet H, Amamou M (2006) Influence of activated charcoal on the pharmacokinetics and the clinical features of carbamazepine poisoning. Am J Emerg Med 24:440-443

276. Pitetti RD, Singh S, Pierce MC (2003) Safe and efficacious use of procedural sedation and analgesia by nonanesthesiologists in a pediatric emergency department. Arch Pediatr Adolesc Med 157:1090-1096

277. Treatment of benzodiazepine overdose with flumazenil (1992) The Flumazenil in Benzodiazepine Intoxication Multicenter Study Group. Clin Ther 14:978-995

278. Lheureux P, Vranckx M, Leduc D, Askenasi R (1992) Flumazenil in mixed benzodiazepine/ tricyclic antidepressant overdose: a placebocontrolled study in the dog. Am J Emerg Med 10:184-188

279. Beauvoir C, Passeron D, du Cailar G, Millet E (1991) [Diltiazem poisoning: hemodynamic aspects]. Ann Fr Anesth Reanim 10:154-157

280. Gillart T, Loiseau S, Azarnoush K, Gonzalez D, Guelon D (2008) [Resuscitation after three hours of cardiac arrest with severe hypothermia following a toxic coma]. Ann Fr Anesth Reanim 27:510-513

281. Nordt SP, Clark RF (1997) Midazolam: a review of therapeutic uses and toxicity. J Emerg Med 15:357-365

282. Machin KL, Caulkett NA (1998) Cardiopulmonary effects of propofol and a medetomidinemidazolam-ketamine combination in mallard ducks. Am J Vet Res 59:598-602

283. Osterwalder JJ (1996) Naloxone-for intoxications with intravenous heroin and heroin mixturesharmless or hazardous? A prospective clinical study. J Toxicol Clin Toxicol 34:409-416

284. Sporer KA, Firestone J, Isaacs SM (1996) Out-ofhospital treatment of opioid overdoses in an urban setting. Acad Emerg Med 3:660-667

285. Wanger K, Brough L, Macmillan I, Goulding J, MacPhail I, Christenson JM (1998) Intravenous vs subcutaneous naloxone for out-of-hospital management of presumed opioid overdose. Acad Emerg Med 5:293-299

286. Hasan RA, Benko AS, Nolan BM, Campe J, Duff J, Zureikat GY (2003) Cardiorespiratory effects of naloxone in children. Ann Pharmacother 37:1587-1592

287. Sporer KA (1999) Acute heroin overdose. Ann Intern Med 130:584-590

288. Kaplan JL, Marx JA, Calabro JJ et al (1999) Double-blind, randomized study of nalmefene and naloxone in emergency department patients with suspected narcotic overdose. Ann Emerg Med 34:42-50
289. Schneir AB, Vadeboncoeur TF, Offerman SR et al (2002) Massive OxyContin ingestion refractory to naloxone therapy. Ann Emerg Med 40:425-428

290. Kelly AM, Kerr D, Dietze P, Patrick I, Walker T, Koutsogiannis Z (2005) Randomised trial of intranasal versus intramuscular naloxone in prehospital treatment for suspected opioid overdose. Med J Aust 182:24-27

291. Robertson TM, Hendey GW, Stroh G, Shalit M (2009) Intranasal naloxone is a viable alternative to intravenous naloxone for prehospital narcotic overdose. Prehosp Emerg Care 13:512-515

292. Kerr D, Kelly AM, Dietze P, Jolley D, Barger B (2009) Randomized controlled trial comparing the effectiveness and safety of intranasal and intramuscular naloxone for the treatment of suspected heroin overdose. Addiction 104:20672074

293. Barton ED, Colwell CB, Wolfe T et al (2005) Efficacy of intranasal naloxone as a needleless alternative for treatment of opioid overdose in the prehospital setting. J Emerg Med 29:265-271

294. Boyd JJ, Kuisma MJ, Alaspaa AO, Vuori E, Repo JV, Randell TT (2006) Recurrent opioid toxicity after pre-hospital care of presumed heroin overdose patients. Acta Anaesthesiol Scand 50:1266-1270

295. Buajordet I, Naess AC, Jacobsen D, Brors O (2004) Adverse events after naloxone treatment of episodes of suspected acute opioid overdose. Eur J Emerg Med 11:19-23

296. Cantwell K, Dietze P, Flander L (2005) The relationship between naloxone dose and key patient variables in the treatment of non-fatal heroin overdose in the prehospital setting. Resuscitation 65:315-319

297. Cetrullo C, Di Nino GF, Melloni C, Pieri C, Zanoni A (1983) Naloxone antagonism toward opiate analgesic drugs. Clinical experimental study. Minerva Anestesiol 49:199-204

298. Nielsen K, Nielsen SL, Siersma V, Rasmussen LS (2011) Treatment of opioid overdose in a physician-based prehospital EMS: frequency and long-term prognosis. Resuscitation 82:1410 1413

299. Stokland O, Hansen TB, Nilsen JE (1998) Prehospital treatment of heroin intoxication in Oslo in 1996. Tidsskr Nor Laegeforen 118:31443146

300. Wampler DA, Molina DK, McManus J, Laws P, Manifold CA (2011) No deaths associated with patient refusal of transport after naloxonereversed opioid overdose. Prehosp Emerg Care 15:320-324

301. Tokarski GF, Young MJ (1988) Criteria for admitting patients with tricyclic antidepressant overdose. J Emerg Med 6:121-124

302. Banahan BF Jr, Schelkun PH (1990) Tricyclic antidepressant overdose: conservative management in a community hospital with costsaving implications. J Emerg Med 8:451-454

303. Hulten BA, Adams R, Askenasi R et al (1992) Predicting severity of tricyclic antidepressant overdose. J Toxicol Clin Toxicol 30:161-170

304. Bailey B, Buckley NA, Amre DK (2004) A metaanalysis of prognostic indicators to predict seizures, arrhythmias or death after tricyclic antidepressant overdose. J Toxicol Clin Toxicol 42:877-888

305. Thanacoody HK, Thomas SH (2005) Tricyclic antidepressant poisoning: cardiovascular toxicity. Toxicol Rev 24:205-214 
306. Woolf AD, Erdman AR, Nelson LS et al (2007) Tricyclic antidepressant poisoning: an evidencebased consensus guideline for out-of-hospital management. Clin Toxicol (Phila) 45:203-233

307. Hoffman JR, Votey SR, Bayer M, Silver L (1993) Effect of hypertonic sodium bicarbonate in the treatment of moderate-to-severe cyclic antidepressant overdose. Am J Emerg Med 11:336-341

308. Koppel C, Wiegreffe A, Tenczer J (1992) Clinical course, therapy, outcome and analytical data in amitriptyline and combined amitriptyline/ chlordiazepoxide overdose. Hum Exp Toxicol 11:458-465

309. Hedges JR, Baker PB, Tasset JJ, Otten EJ, Dalsey WC, Syverud SA (1985) Bicarbonate therapy for the cardiovascular toxicity of amitriptyline in an animal model. J Emerg Med 3:253-260

310. Knudsen K, Abrahamsson J (1997) Epinephrine and sodium bicarbonate independently and additively increase survival in experimental amitriptyline poisoning. Crit Care Med 25:669674

311. Sasyniuk BI, Jhamandas V, Valois M (1986) Experimental amitriptyline intoxication: treatment of cardiac toxicity with sodium bicarbonate. Ann Emerg Med 15:1052-1059

312. Bradberry SM, Thanacoody HK, Watt BE, Thomas SH, Vale JA (2005) Management of the cardiovascular complications of tricyclic antidepressant poisoning: role of sodium bicarbonate. Toxicol Rev 24:195-204

313. Yoav G, Odelia G, Shaltiel C (2002) A lipid emulsion r(ed)uces mortality from clomipramine overdose in rats. Vet Hum Toxicol 44:30

314. Harvey M, Cave G (2007) Intralipid outperforms sodium bicarbonate in a rabbit model of clomipramine toxicity. Annals of emergency medicine 49:178-185, 85 e1-4

315. Brunn GJ, Keyler DE, Pond SM, Pentel PR (1992) Reversal of desipramine toxicity in rats using drug-specific antibody Fab' fragment: effects on hypotension and interaction with sodium bicarbonate. J Pharmacol Exp Ther 260:1392-1399

316. Brunn GJ, Keyler DE, Ross CA, Pond SM, Pentel PR (1991) Drug-specific F(ab')2 fragment r(ed) uces desipramine cardiotoxicity in rats. Int J Immunopharmacol 13:841-851

317. Hursting MJ, Opheim KE, Raisys VA, Kenny MA, Metzger G (1989) Tricyclic antidepressantspecific Fab fragments alter the distribution and elimination of desipramine in the rabbit: a model for overdose treatment. J Toxicol Clin Toxicol 27:53-66

318. Pentel PR, Scarlett W, Ross CA, Landon J, Sidki A, Keyler DE (1995) Reduction of desipramine cardiotoxicity and prolongation of survival in rats with the use of polyclonal drug-specific antibody Fab fragments. Ann Emerg Med 26:334-341

319. Pentel PR, Ross CA, Landon J, Sidki A, Shelver WL, Keyler DE (1994) Reversal of desipramine toxicity in rats with polyclonal drug-specific antibody Fab fragments. J Lab Clin Med 123:387-393

320. Dart RC, Sidki A, Sullivan JB Jr, Egen NB, Garcia RA (1996) Ovine desipramine antibody fragments reverse desipramine cardiovascular toxicity in the rat. Ann Emerg Med 27:309-315

321. Heard K, Dart RC, Bogdan G et al (2006) A preliminary study of tricyclic antidepressant (TCA) ovine FAB for TCA toxicity. Clin Toxicol (Phila) 44:275-281
322. Pentel P, Peterson CD (1980) Asystole complicating physostigmine treatment of tricyclic antidepressant overdose. Ann Emerg Med 9:588-590

323. Lange RA, Cigarroa RG, Yancy CW Jr et al (1989) Cocaine-induced coronary-artery vasoconstriction. N Engl J Med 321:1557-1562

324. Baumann BM, Perrone J, Hornig SE, Shofer FS, Hollander JE (2000) Randomized, double-blind placebo-controlled trial of diazepam, nitroglycerin, or both for treatment of patients with potential cocaine-associated acute coronary syndromes. Acad Emerg Med 7:878-885

325. Honderick T, Williams D, Seaberg D, Wears $R$ (2003) A prospective, randomized, controlled trial of benzodiazepines and nitroglycerine or nitroglycerine alone in the treatment of cocaineassociated acute coronary syndromes. Am J Emerg Med 21:39-42

326. Negus BH, Willard JE, Hillis LD et al (1994) Alleviation of cocaine-induced coronary vasoconstriction with intravenous verapamil. Am J Cardiol 73:510-513

327. Saland KE, Hillis LD, Lange RA, Cigarroa JE (2002) Influence of morphine sulfate on cocaineinduced coronary vasoconstriction. Am J Cardiol 90:810-811

328. Brogan WCI, Lange RA, Kim AS, Moliterno DJ, Hillis LD (1991) Alleviation of cocaine-induced coronary vasoconstriction by nitroglycerin. J Am Coll Cardiol 18:581-586

329. Hollander JE, Hoffman RS, Gennis P et al (1994) Nitroglycerin in the treatment of cocaine associated chest pain-clinical safety and efficacy. Joxicol Clin Toxicol 32:243-256

330. Dattilo PB, Hailpern SM, Fearon K, Sohal D, Nordin C (2008) Beta-blockers are associated with reduced risk of myocardial infarction after cocaine use. Ann Emerg Med 51:117-125

331. Vongpatanasin W, Mansour Y, Chavoshan B, Arbique D, Victor RG (1999) Cocaine stimulates the human cardiovascular system via a central mechanism of action. Circulation 100:497-502

332. Lange RA, Cigarroa RG, Flores ED et al (1990) Potentiation of cocaine-induced coronary vasoconstriction by beta-adrenergic blockade. Ann Intern Med 112:897-903

333. Sand IC, Brody SL, Wrenn KD, Slovis CM (1991) Experience with esmolol for the treatment of cocaine-associated cardiovascular complications. Am J Emerg Med 9:161-163

334. Sofuoglu M, Brown S, Babb DA, Pentel PR, Hatsukami DK (2000) Carvedilol affects the physiological and behavioral response to smoked cocaine in humans. Drug Alcohol Depend 60:69-76

335. Sofuoglu M, Brown S, Babb DA, Pentel PR, Hatsukami DK (2000) Effects of labetalol treatment on the physiological and subjective response to smoked cocaine. Pharmacol Biochem Behav 65:255-259

336. Boehrer JD, Moliterno DJ, Willard JE, Hillis LD, Lange RA (1993) Influence of labetalol on cocaine-induced coronary vasoconstriction in humans. Am J Med 94:608-610

337. Hsue PY, McManus D, Selby V et al (2007) Cardiac arrest in patients who smoke crack cocaine. Am Cardiol 99:822-824

338. Litz RJ, Popp M, Stehr SN, Koch T (2006) Successful resuscitation of a patient with ropivacaine-induced asystole after axillary plexus block using lipid infusion. Anaesthesia 61:800801
339. Rosenblatt MA, Abel M, Fischer GW, Itzkovich CJ, Eisenkraft JB (2006) Successful use of a $20 \%$ lipid emulsion to resuscitate a patient after a presumed bupivacaine-related cardiac arrest. Anesthesiology 105:217-218

340. Marwick PC, Levin Al, Coetzee AR (2009) Recurrence of cardiotoxicity after lipid rescue from bupivacaine-induced cardiac arrest. Anesth Analg 108:1344-1346

341. Warren JA, Thoma RB, Georgescu A, Shah SJ (2008) Intravenous lipid infusion in the successful resuscitation of local anesthetic-induced cardiovascular collapse after supraclavicular brachial plexus block. Anesth Analg 106:15781580. (table of contents)

342. Smith HM, Jacob AK, Segura LG, Dilger JA, Torsher LC (2008) Simulation education in anesthesia training: a case report of successful resuscitation of bupivacaine-induced cardiac arrest linked to recent simulation training. Anesth Analg 106:1581-1584. (table of contents)

343. Foxall GL, Hardman JG, Bedforth NM (2007) Three-dimensional, multiplanar, ultrasoundguided radial nerve block. Reg Anesth Pain Med 32:516-521

344. Shah S, Gopalakrishnan S, Apuya J, Martin T (2009) Use of Intralipid in an infant with impending cardiovascular collapse due to local anesthetic toxicity. J Anesth 23:439-441

345. Zimmer C, Piepenbrink K, Riest G, Peters J (2007) [Cardiotoxic and neurotoxic effects after accidental intravascular bupivacaine administration. Therapy with lidocaine propofol and lipid emulsion]. Anaesthesist 56:449-453

346. Litz RJ, Roessel T, Heller AR, Stehr SN (2008) Reversal of central nervous system and cardiac toxicity after local anesthetic intoxication by lipid emulsion injection. Anesth Analg 106:15751577. (table of contents)

347. Ludot H, Tharin JY, Belouadah M, Mazoit JX, Malinovsky JM (2008) Successful resuscitation after ropivacaine and lidocaine-induced ventricular arrhythmia following posterior lumbar plexus block in a child. Anesth Analg 106:1572-1574. (table of contents)

348. Cave G, Harvey MG, Winterbottom T (2009) Evaluation of the Association of Anaesthetists of Great Britain and Ireland lipid infusion protocol in bupivacaine induced cardiac arrest in rabbits. Anaesthesia 64:732-737

349. Di Gregorio GS, Ripper R et al (2009) Lipid emulsion is superior to vasopressin in a rodent model of resuscitation from toxin-induced cardiac arrest. Crit Care Med 37:993-999

350. Weinberg GL, VadeBoncouer T, Ramaraju GA, Garcia-Amaro MF, Cwik MJ (1998) Pretreatment or resuscitation with a lipid infusion shifts the dose-response to bupivacaine-induced asystole in rats. Anesthesiology 88:1071-1075

351. Weinberg G, Ripper R, Feinstein DL, Hoffman W (2003) Lipid emulsion infusion rescues dogs from bupivacaine-induced cardiac toxicity. Reg Anesth Pain Med 28:198-202

352. Weinberg GL, Di Gregorio G, Ripper R et al (2008) Resuscitation with lipid versus epinephrine in a rat model of bupivacaine overdose. Anesthesiology 108:907-913

353. 2010 Management of Severe Local Anaesthetic Toxicity. Association of Anaesthetists of Great Britain and Ireland, (Accessed 28 June 2010, 2010) 
354. Mayr VD, Mitterschiffthaler L, Neurauter A et al (2008) A comparison of the combination of epinephrine and vasopressin with lipid emulsion in a porcine model of asphyxial cardiac arrest after intravenous injection of bupivacaine. Anesth Analg 106:1566-1571. (table of contents)

355. Hicks SD, Salcido DD, Logue ES et al (2009) Lipid emulsion combined with epinephrine and vasopressin does not improve survival in a swine model of bupivacaine-induced cardiac arrest. Anesthesiology 111:138-146

356. Hiller DB, Gregorio GD, Ripper R et al (2009) Epinephrine impairs lipid resuscitation from bupivacaine overdose: a threshold effect. Anesthesiology 111:498-505

357. Bailey B (2003) Glucagon in beta-blocker and calcium channel blocker overdoses: a systematic review. J Toxicol Clin Toxicol 41:595-602

358. Fahed S, Grum DF, Papadimos TJ (2008) Labetalol infusion for refractory hypertension causing severe hypotension and bradycardia: an issue of patient safety. Patient Saf Surg 2:13

359. Fernandes CM, Daya MR (1995) Sotalol-induced bradycardia reversed by glucagon. Can Fam Physician 41:659-660, 63-65

360. Frishman W, Jacob $H$, Eisenberg $E$, Ribner $H$ (1979) Clinical pharmacology of the new betaadrenergic blocking drugs. Part 8. Self-poisoning with beta-adrenoceptor blocking agents: recognition and management. Am Heart J 98:798-811

361. Gabry AL, Pourriat JL, Hoang TD, Lapandry C (1985) [Cardiogenic shock caused by metoprolol poisoning. Reversibility with high doses of glucagon and isoproterenol]. Presse Med 14:229

362. Hazouard E, Ferrandiere M, Lesire V, Joye F, Perrotin D, de Toffol B (1999) Peduncular hallucinosis related to propranolol selfpoisoning: efficacy of intravenous glucagon. Inten Care Med 25:336-337

363. Khan MI, Miller MT (1985) Beta-blocker toxicity the role of glucagon. Report of 2 cases. S Afr Med J 67:1062-1063

364. Letter MBH (1976) Massive intoxication with metoprolol. Br Med J 1:222

365. O'Mahony D, O'Leary P, Molloy MG (1990) Severe oxprenolol poisoning: the importance of glucagon infusion. Hum Exp Toxicol 9:101-103

366. Wallin CJ, Hulting J (1983) Massive metoprolol poisoning treated with prenalterol. Acta Med Scand 214:253-255

367. Weinstein RS, Cole S, Knaster HB, Dahlbert T (1985) Beta blocker overdose with propranolol and with atenolol. Ann Emerg Med 14:161-163

368. Alderfliegel F, Leeman $M$, Demaeyer $P$, Kahn RJ (1993) Sotalol poisoning associated with asystole. Inten Care Med 19:57-58

369. Kenyon CJ, Aldinger GE, Joshipura P, Zaid GJ (1988) Successful resuscitation using external cardiac pacing in beta adrenergic antagonistinduced bradyasystolic arrest. Ann Emerg Med 17:711-713

370. Freestone S, Thomas HM, Bhamra RK, Dyson EH (1986) Severe atenolol poisoning: treatment with prenalterol. Hum Toxicol 5:343-345

371. Kerns W 2nd, Schroeder D, Williams C, Tomaszewski C, Raymond R (1997) Insulin improves survival in a canine model of acute beta-blocker toxicity. Ann Emerg Med 29:748757

372. Holger JS, Engebretsen KM, Fritzlar SJ, Patten LC, Harris CR, Flottemesch TJ (2007) Insulin versus vasopressin and epinephrine to treat betablocker toxicity. Clin Toxicol (Phila) 45:396-401
373. Page C, Hacket LP, Isbister GK (2009) The use of high-dose insulin-glucose euglycemia in betablocker overdose: a case report. J Med Toxicol 5:139-143

374. Jovic-Stosic J, Gligic B, Putic V, Brajkovic G, Spasic R (2011) Severe propranolol and ethanol overdose with wide complex tachycardia treated with intravenous lipid emulsion: a case report. Clin Toxicol (Phila) 49:426-430

375. Barton CA, Johnson NB, Mah ND, Beauchamp G Hendrickson R (2015) Successful treatment of a massive metoprolol overdose using intravenous lipid emulsion and hyperinsulinemia/euglycemia therapy. Pharmacotherapy 35:e56-e60

376. Sebe A, Disel NR, Acikalin Akpinar A, Karakoc E (2015) Role of intravenous lipid emulsions in the management of calcium channel blocker and beta-blocker overdose: 3 years experience of a university hospital. Postgrad Med 127:119-124

377. Doepker B, Healy W, Cortez E, Adkins EJ (2014) High-dose insulin and intravenous lipid emulsion therapy for cardiogenic shock induced by intentional calcium-channel blocker and Betablocker overdose: a case series. J Emerg Med 46:486-490

378. Kollef MH (1994) Labetalol overdose successfully treated with amrinone and alpha-adrenergic receptor agonists. Chest 105:626-627

379. O'Grady J, Anderson S, Pringle D (2001) Successful treatment of severe atenolol overdose with calcium chloride. Cjem 3:224-227

380. McVey FK, Corke CF (1991) Extracorporeal circulation in the management of massive propranolol overdose. Anaesthesia 46:744-746

381. Lane AS, Woodward AC, Goldman MR (1987) Massive propranolol overdose poorly responsive to pharmacologic therapy: use of the intra-aortic balloon pump. Ann Emerg Med 16:1381-1383

382. Rooney M, Massey KL, Jamali F, Rosin M, Thomson D, Johnson DH (1996) Acebutolol overdose treated with hemodialysis and extracorporeal membrane oxygenation. J Clin Pharmacol 36:760-763

383. Brimacombe JR, Scully M, Swainston R (1991) Propranolol overdose-a dramatic response to calcium chloride. Med J Aust 155:267-268

384. Bronstein AC, Spyker DA, Cantilena LR Jr, Green JL, Rumack BH, Giffin SL (2009) 2008 Annual Report of the American Association of Poison Control Centers' National Poison Data System (NPDS): 26th Annual Report. Clin Toxicol (Phila) 47:911-1084

385. Olson KR, Erdman AR, Woolf AD et al (2005) Calcium channel blocker ingestion: an evidencebased consensus guideline for out-of-hospital management. Clin Toxicol (Phila) 43:797-822

386. St-Onge M, Dube PA, Gosselin S et al (2014) Treatment for calcium channel blocker poisoning: a systematic review. Clin Toxicol (Phila) 52:926-944

387. Levine M, Curry SC, Padilla-Jones A, Ruha AM (2013) Critical care management of verapamil and diltiazem overdose with a focus on vasopressors: a 25 -year experience at a single center. Ann Emerg Med 62:252-258

388. Cohen V, Jellinek SP, Fancher L et al (2009) Tarka(R) (Trandolapril/Verapamil Hydrochloride Extended-Release) overdose. J Emerg Med 40(3):291-295

389. Greene SL, Gawarammana I, Wood DM, Jones AL, Dargan PI (2007) Relative safety of hyperinsulinaemia/euglycaemia therapy in the management of calcium channel blocker overdose: a prospective observational study. Inten Care Med 33:2019-2024
390. Harris NS (2006) Case records of the Massachusetts General Hospital. Case 24-2006. A 40-year-old woman with hypotension after an overdose of amlodipine. N Engl J Med 355:602611

391. Johansen KK, Belhage B (2007) [A 48-year-old woman's survival from a massive verapamil overdose]. Ugeskr Laeger 169:4074-4075

392. Kanagarajan K, Marraffa JM, Bouchard NC, Krishnan P, Hoffman RS, Stork CM (2007) The use of vasopressin in the setting of recalcitrant hypotension due to calcium channel blocker overdose. Clin Toxicol (Phila) 45:56-59

393. Marques M, Gomes E, de Oliveira J (2003) Treatment of calcium channel blocker intoxication with insulin infusion: case report and literature review. Resuscitation 57:211-213

394. Meyer M, Stremski E, Scanlon M (2003) Successful resuscitation of a verapamil intoxicated child with a dextrose-insulin infusion. Clin Inten Care 14:109-113

395. Ortiz-Munoz L, Rodriguez-Ospina LF, FigueroaGonzalez M (2005) Hyperinsulinemic-euglycemic therapy for intoxication with calcium channe blockers. Bol Asoc Med P R 97:182-189

396. Patel NP, Pugh ME, Goldberg S, Eiger G (2007) Hyperinsulinemic euglycemia therapy for verapamil poisoning: case report. Am J Crit Care 16:520, 18-19

397. Rasmussen L, Husted SE, Johnsen SP (2003) Severe intoxication after an intentional overdose of amlodipine. Acta Anaesthesiol Scand 47:1038-1040

398. Smith SW, Ferguson KL, Hoffman RS, Nelson LS, Greller HA (2008) Prolonged severe hypotension following combined amlodipine and valsartan ingestion. Clin Toxicol (Phila) 46:470-474

399. Eddleston M, Rajapakse S, Rajakanthan et al (2000) Anti-digoxin Fab fragments in cardiotoxicity induced by ingestion of yellow oleander: a randomised controlled trial. Lancet 355:967-972

400. Lapostolle F, Borron SW, Verdier C et al (2008) Digoxin-specific Fab fragments as single firstline therapy in digitalis poisoning. Crit Care Med 36:3014-3018

401. Chan BS, Buckley NA (2014) Digoxin-specific antibody fragments in the treatment of digoxin toxicity. Clin Toxicol (Phila) 52:824-836

402. Dasgupta A, Szelei-Stevens KA (2004) Neutralization of free digoxin-like immunoreactive components of oriental medicines Dan Shen and Lu-Shen-Wan by the Fab fragment of antidigoxin antibody (Digibind). Am J Clin Pathol 121:276-281

403. Bosse GM, Pope TM (1994) Recurrent digoxin overdose and treatment with digoxin-specific Fab antibody fragments. J Emerg Med 12:179185

404. Borron SW, Baud FJ, Barriot P, Imbert M, Bismuth C (2007) Prospective study of hydroxocobalamin for acute cyanide poisoning in smoke inhalation. Ann Emerg Med 49:794-801, e1-2

405. Espinoza OB, Perez M, Ramirez MS (1992) Bitter cassava poisoning in eight children: a case report. Vet Hum Toxicol 34:65

406. Houeto $P$, Hoffman JR, Imbert $M$, Levillain $P$, Baud FJ (1995) Relation of blood cyanide to plasma cyanocobalamin concentration after a fixed dose of hydroxocobalamin in cyanide poisoning. The Lancet 346:605-608 
407. Pontal P, Bismuth C, Garnier R (1982) Therapeutic attitude in cyanide poisoning: Retrospective study of 24 non-lethal cases. Vet Hum Toxicol 24:286-287

408. Kirk MA, Gerace R, Kulig KW (1993) Cyanide and methemoglobin kinetics in smoke inhalation victims treated with the cyanide antidote kit. Ann Emerg Med 22:1413-1418

409. Chen KK, Rose CL (1952) Nitrite and thiosulfate therapy in cyanide poisoning. J Am Med Assoc 149:113-119

410. Yen D, Tsai J, Wang LM et al (1995) The clinical experience of acute cyanide poisoning. Am J Emerg Med 13:524-528

411. Reade MC, Davies SR, Morley PT, Dennett J, Jacobs IC, Australian Resuscitation C (2012) Review article: management of cyanide poisoning. Emerg Med Australas 24:225-238

412. Streitz MJ, Bebarta VS, Borys DJ, Morgan DL (2014) Patterns of cyanide antidote use since regulatory approval of hydroxocobalamin in the United States. Am J Ther 21:244-249

413. Dries DJ, Endorf FW (2013) Inhalation injury: epidemiology, pathology, treatment strategies. Scan J Trauma Resusc Emerg Med 21:31

414. Iqbal S, Clower JH, Boehmer TK, Yip FY, Garbe P (2010) Carbon monoxide-related hospitalizations in the U.S.: evaluation of a web-based query system for public health surveillance. Public Health Rep 125:423-432

415. Hampson NB, Hauff NM (2008) Carboxyhemoglobin levels in carbon monoxide poisoning: do they correlate with the clinical picture? Am J Emerg Med 26:665-669

416. Buckley NA, Juurlink DN, Isbister G, Bennett MH, Lavonas EJ (2011) Hyperbaric oxygen for carbon monoxide poisoning. Cochrane Database Syst Rev 4:CD002041

417. Weaver LK (2009) Clinical practice. Carbon monoxide poisoning. N Engl J Med 360:12171225

418. Juurlink DN, Buckley NA, Stanbrook MB, Isbister GK, Bennett M, McGuigan MA (2005) Hyperbaric oxygen for carbon monoxide poisoning. Cochrane Database Syst Rev 1:CD002041

419. Roderique JD, Josef CS, Feldman MJ, Spiess BD (2015) A modern literature review of carbon monoxide poisoning theories, therapies, and potential targets for therapy advancement. Toxicology 334:45-58

420. Satran D, Henry CR, Adkinson C, Nicholson Cl, Bracha Y, Henry TD (2005) Cardiovascular manifestations of moderate to severe carbon monoxide poisoning. J Am Coll Cardiol 45:15131516

421. Henry CR, Satran D, Lindgren B, Adkinson C, Nicholson Cl, Henry TD (2006) Myocardial injury and long-term mortality following moderate to severe carbon monoxide poisoning. JAMA 295:398-402

422. Braz LG, Modolo NS, do Nascimento P Jr et al (2006) Perioperative cardiac arrest: a study of 53,718 anaesthetics over $9 \mathrm{yr}$ from a Brazilian teaching hospital. Br J Anaesth 96:569-575

423. Sprung J, Warner ME, Contreras MG et al (2003) Predictors of survival following cardiac arrest in patients undergoing noncardiac surgery: a study of 518,294 patients at a tertiary referral center. Anesthesiology 99:259-269

424. Nunnally ME, O'Connor MF, Kordylewski H, Westlake B, Dutton RP (2015) The incidence and risk factors for perioperative cardiac arrest observed in the national anesthesia clinical outcomes registry. Anesth Analg 120:364-370
425. Nunes JC, Braz JR, Oliveira TS, de Carvalho LR, Castiglia YM, Braz LG (2014) Intraoperative and anesthesia-related cardiac arrest and its mortality in older patients: a 15-year survey in a tertiary teaching hospital. PloS one 9:e104041

426. Siriphuwanun V, Punjasawadwong $Y$ Lapisatepun W, Charuluxananan S, Uerpairojkit K (2014) Incidence of and factors associated with perioperative cardiac arrest within 24 hours of anesthesia for emergency surgery. Risk Manag Healthc Policy 7:155-162

427. Gonzalez LP, Braz JR, Modolo MP, de Carvalho LR, Modolo NS, Braz LG (2014) Pediatric perioperative cardiac arrest and mortality: a study from a tertiary teaching hospital. Pediatr Crit Care Med 15:878-884

428. Ellis SJ, Newland MC, Simonson JA et al (2014) Anesthesia-related cardiac arrest. Anesthesiology 120:829-838

429. Newland MC, Ellis SJ, Lydiatt CA et al (2002) Anesthetic-related cardiac arrest and its mortality: a report covering 72,959 anesthetics over 10 years from a US teaching hospital. Anesthesiology 97:108-115

430. Bhananker SM, Ramamoorthy C, Geiduschek JM et al (2007) Anesthesia-related cardiac arrest in children: update from the Pediatric Perioperative Cardiac Arrest Registry. Anesth Analg 105:344350

431. Krishna Ramachandran SM, Kheterpal S et al (2013) Predictors of survival from perioperative cardiopulmonary arrests: a retrospective analysis of 2,524 events from the Get With The Guidelines-Resuscitation registry. Anesthesiology 119:1322-1339

432. Brown J, Rogers J, Soar J (2001) Cardiac arrest during surgery and ventilation in the prone position: a case report and systematic review. Resuscitation 50:233-238

433. Atkinson MC (2000) The efficacy of cardiopulmonary resuscitation in the prone position. Crit Care Resusc 2:188-190

434. Mertes PM, Tajima K, Regnier-Kimmoun MA et al (2010) Perioperative anaphylaxis. Med Clin North Am 94:761-789, xi

435. Wolfe JW, Butterworth JF (2011) Local anesthetic systemic toxicity: update on mechanisms and treatment. Curr Opin Anaesthesiol 24:561-566

436. Cao D, Heard K, Foran M, Koyfman A (2015) Intravenous lipid emulsion in the emergency department: a systematic review of recent literature. J Emerg Med 48:387-397

437. Waring WS (2012) Intravenous lipid administration for drug-induced toxicity: a critical review of the existing data. Expert Rev Clin Pharmacol 5:437-444

438. Neal JM, Mulroy MF, Weinberg GL American Society of Regional A, Pain M (2012) American Society of Regional Anesthesia and Pain Medicine checklist for managing local anesthetic systemic toxicity: 2012 version. Reg Anesth Pain Med 37:16-18

439. Mazer SP, Weisfeldt M, Bai D et al (2003) Reverse CPR: a pilot study of CPR in the prone position. Resuscitation 57:279-285

440. Meaney PA, Bobrow BJ, Mancini ME et al (2013) Cardiopulmonary resuscitation quality: improving cardiac resuscitation outcomes both inside and outside the hospital: a consensus statement from the american heart association. Circulation 128:417-435
441. Martin GB, Carden DL, Nowak RM, Lewinter JR, Johnston W, Tomlanovich MC (1986) Aortic and right atrial pressures during standard and simultaneous compression and ventilation CPR in human beings. Ann Emerg Med 15:125-130

442. Timerman S, Cardoso LF, Ramires JA, Halperin $H$ (2004) Improved hemodynamic performance with a novel chest compression device during treatment of in-hospital cardiac arrest. Resuscitation 61:273-280

443. Niemann JT, Rosborough JP, Ung S, Criley JM (1982) Coronary perfusion pressure during experimental cardiopulmonary resuscitation. Ann Emerg Med 11:127-131

444. Nolan JP, Neumar RW, Adrie C et al (2010) Post-cardiac arrest syndrome: epidemiology, pathophysiology, treatment, and prognostication: a scientific statement from the International Liaison Committee on Resuscitation; the American Heart Association Emergency Cardiovascular Care Committee; the Council on Cardiovascular Surgery and Anesthesia; the Council on Cardiopulmonary, Perioperative, and Critical Care; the Council on Clinical Cardiology; the Council on Stroke (Part II). Int Emerg Nurs 18:8-28

445. British Medical Association the Resuscitation Council (UK) and the Royal College of Nursing (2014) Decisions relating to cardiopulmonary resuscitation. A joint statment from the British Medical Association, the Resuscitation Council (UK) and the Royal College of Nursing. British Medical Association, London

446. Charalambous CP, Zipitis CS, Keenan DJ (2006) Chest reexploration in the intensive care unit after cardiac surgery: a safe alternative to returning to the operating theater. Ann Thorac Surg 81:191-194

447. McKowen RL, Magovern GJ, Liebler GA, Park SB, Burkholder JA, Maher TD (1985) Infectious complications and cost-effectiveness of open resuscitation in the surgical intensive care unit after cardiac surgery. Ann Thorac Surg 40:388392

448. Pottle A, Bullock I, Thomas J, Scott L (2002) Survival to discharge following Open Chest Cardiac Compression (OCCC). A 4-year retrospective audit in a cardiothoracic specialist centre - Royal Brompton and Harefield NHS Trust, United Kingdom. Resuscitation 52:269-272

449. Mackay JH, Powell SJ, Osgathorp J, Rozario CJ (2002) Six-year prospective audit of chest reopening after cardiac arrest. Eur J Cardiothorac Surg 22:421-425

450. Birdi I, Chaudhuri N, Lenthall K, Reddy S, Nashef SA (2000) Emergency reinstitution of cardiopulmonary bypass following cardiac surgery: outcome justifies the cost. Eur J Cardiothorac Surg 17:743-746

451. el-Banayosy A, Brehm C, Kizner L et al (1998) Cardiopulmonary resuscitation after cardiac surgery: a two-year study. J Cardiothorac Vasc Anesth 12:390-392

452. Anthi A, Tzelepis GE, Alivizatos P, Michalis A, Palatianos GM, Geroulanos S (1998) Unexpected cardiac arrest after cardiac surgery: Incidence, predisposing causes, and outcome of open chest cardiopulmonary resuscitation. Chest 113:15-19

453. Wahba A, Gotz W, Birnbaum DE (1997) Outcome of cardiopulmonary resuscitation following open heart surgery. Scand Cardiovasc J 31:147-149

454. Kaiser GC, Naunheim KS, Fiore AC et al (1990) Reoperation in the intensive care unit. Ann Thorac Surg 49:903-907 (discussion 8) 
455. LaPar DJ, Ghanta RK, Kern JA et al (2014) Hospital variation in mortality from cardiac arrest after cardiac surgery: an opportunity for improvement? Ann Thorac Surg 98:534-539 (discussion 9-40)

456. Rhodes JF, Blaufox AD, Seiden HS et al (1999) Cardiac arrest in infants after congenital heart surgery. Circulation 100:II194-II199

457. Kempen PM, Allgood R (1999) Right ventricular rupture during closed-chest cardiopulmonary resuscitation after pneumonectomy with pericardiotomy: a case report. Crit Care Med 27:1378-1379

458. Bohrer H, Gust R, Bottiger BW (1995) Cardiopulmonary resuscitation after cardiac surgery. J Cardiothorac Vasc Anesth 9:352

459. Klintschar M, Darok M, Radner H (1998) Massive injury to the heart after attempted active compression-decompression cardiopulmonary resuscitation. Int J Legal Med 111:93-96

460. Fosse $E$, Lindberg $H$ (1996) Left ventricular rupture following external chest compression. Acta Anaesthesiol Scand 40:502-504

461. Li Y, Wang H, Cho JH et al (2010) Defibrillation delivered during the upstroke phase of manual chest compression improves shock success. Crit Care Med 38:910-915

462. Li Y, Yu T, Ristagno G et al (2010) The optimal phasic relationship between synchronized shock and mechanical chest compressions. Resuscitation 81:724-729

463. Larsen Al, Hjornevik AS, Ellingsen CL, Nilsen DW (2007) Cardiac arrest with continuous mechanical chest compression during percutaneous coronary intervention. A report on the use of the LUCAS device. Resuscitation 75:454-459

464. Tsao NW, Shih CM, Yeh JS et al (2012) Extracorporeal membrane oxygenation-assisted primary percutaneous coronary intervention may improve survival of patients with acute myocardial infarction complicated by profound cardiogenic shock. J Crit Care 27:530 e1-11

465. Alpert MA (2011) Sudden cardiac arrest and sudden cardiac death on dialysis: epidemiology, evaluation, treatment, and prevention. Hemodial Int 15(1):S22-S29

466. Sacchetti A, Stuccio N, Panebianco P, Torres M (1999) ED hemodialysis for treatment of renal failure emergencies. Am J Emerg Med 17:305307

467. Putcha N, Allon M (2007) Management of hyperkalemia in dialysis patients. Sem Dial 20:431-439

468. Bleyer AJ, Russell GB, Satko SG (1999) Sudden and cardiac death rates in hemodialysis patients. Kidney Int 55:1553-1559

469. Alfonzo AV, Simpson K, Deighan C, Campbell S, Fox J (2007) Modifications to advanced life support in renal failure. Resuscitation 73:12-28

470. Davis TR, Young BA, Eisenberg MS, Rea TD, Copass MK, Cobb LA (2008) Outcome of cardiac arrests attended by emergency medical services staff at community outpatient dialysis centers. Kidney Int 73:933-939

471. Lafrance JP, Nolin L, Senecal L, Leblanc M (2006) Predictors and outcome of cardiopulmonary resuscitation (CPR) calls in a large haemodialysis unit over a seven-year period. Nephrol Dial Transplant 21:1006-1012

472. Meaney PA, Nadkarni VM, Kern KB, Indik JH, Halperin HR, Berg RA (2010) Rhythms and outcomes of adult in-hospital cardiac arrest. Crit Care Med 38:101-108
473. Girotra S, Nallamothu BK, Spertus JA et al (2012) Trends in survival after in-hospital cardiac arrest. N Engl J Med 367:1912-1920

474. Bird S, Petley GW, Deakin CD, Clewlow F (2007) Defibrillation during renal dialysis: a survey of UK practice and procedural recommendations. Resuscitation 73:347-353

475. Lehrich RW, Pun PH, Tanenbaum ND, Smith SR Middleton JP (2007) Automated external defibrillators and survival from cardiac arrest in the outpatient hemodialysis clinic. J Am Soc Nephrol 18:312-320

476. Arsati F, Montalli VA, Florio FM et al (2010) Brazilian dentists' attitudes about medical emergencies during dental treatment. J Dent Educ 74:661-666

477. Girdler NM, Smith DG (1999) Prevalence of emergency events in British dental practice and emergency management skills of British dentists. Resuscitation 41:159-167

478. (2013) Quality standards for cardiopulmonary resuscitation practice and training. Primary dental care - Quality standards for CPR and training. https://www.resus.org.uk/qualitystandards/primary-dental-care-qualitystandards-for-cpr-and-training/

479. Muller MP, Hansel M, Stehr SN, Weber S, Koch $T$ (2008) A state-wide survey of medical emergency management in dental practices: incidence of emergencies and training experience. Emerg Med J 25:296-300

480. Meechan JG, Skelly AM (1997) Problems complicating dental treatment with local anaesthesia or sedation: prevention and management. Dent Update 24:278-283

481. Jowett NI, Cabot LB (2000) Patients with cardiac disease: considerations for the dental practitioner. Br Dent J 189:297-302

482. Chapman PJ, Penkeyman HW (2002) Successful defibrillation of a dental patient in cardiac arrest. Aust Dent J 47:176-177

483. Absi EG (1987) A cardiac arrest in the dental chair. Br Dent J 163:199-200

484. Fujino H, Yokoyama T, Yoshida K, Suwa K (2010) Using a stool for stabilization of a dental chair when CPR is required. Resuscitation 81:502

485. Laurent F, Segal N, Augustin P (2010) Chest compression: not as effective on dental chair as on the floor. Resuscitation 81:1729 (author reply 30)

486. Lepere AJ, Finn J, Jacobs I (2003) Efficacy of cardiopulmonary resuscitation performed in a dental chair. Aust Dent J 48:244-247

487. Yokoyama T, Yoshida K, Suwa K (2008) Efficacy of external cardiac compression in a dental chair. Resuscitation 79:175-176

488. Segal N, Laurent F, Maman L, Plaisance $P$, Augustin P (2012) Accuracy of a feedback device for cardiopulmonary resuscitation on a dental chair. Emerg Med J 29:890-893

489. Perkins GD, Stephenson BT, Smith CM, Gao F (2004) A comparison between over-the-head and standard cardiopulmonary resuscitation. Resuscitation 61:155-161

490. Handley AJ, Handley JA (2004) Performing chest compressions in a confined space. Resuscitation 61:55-61

491. Maisch S, Issleib M, Kuhls B et al (2010) A comparison between over-the-head and standard cardiopulmonary resuscitation performed by two rescuers: a simulation study. $J$ Emerg Med 39:369-376
492. Chi CH, Tsou JY, Su FC (2009) Comparison of chest compression kinematics associated with over-the-head and standard cardiopulmonary resuscitation. Am J Emerg Med 27:1112-1116

493. Perkins GD, Handley AJ, Koster KW et al (2015) European Resuscitation Council Guidelines for Resuscitation Section 2. Adult basic life support and automated external defibrillation. Resuscitation 95:81-98

494. Rosenberg M (2010) Preparing for medical emergencies: the essential drugs and equipment for the dental office. J Am Dent Assoc 141(Suppl 1):14S-19S

495. (UK) RC (2013) Quality Standards for cardiopulmonary resuscitation practice and training. Acute Care. Resuscitation Council (UK), London

496. Hunter PL (1991) Cardiac arrest in the dental surgery. Br Dent J 170:284

497. Deakin CD, Fothergill R, Moore F, Watson L, Whitbread M (2014) Level of consciousness on admission to a Heart Attack Centre is a predictor of survival from out-of-hospital cardiac arrest. Resuscitation 85:905-909

498. Laurent F, Augustin P, Zak C, Maman L, Segal N (2011) Preparedness of dental practices to treat cardiac arrest: availability of defibrillators. Resuscitation 82:1468-1469

499. Kandray DP, Pieren JA, Benner RW (2007) Attitudes of Ohio dentists and dental hygienists on the use of automated external defibrillators. J Dent Educ 71:480-486

500. (2013) Safe Sedation Practice for Healthcare Procedures: Standards and Guidance. http:// www.aomrc.org.uk/doc_details/9737-safesedation-practice-for-healthcare-proceduresstandards-and-guidance

501. Chapman PJ (1995) A questionnaire survey of dentists regarding knowledge and perceived competence in resuscitation and occurrence of resuscitation emergencies. Aust Dent J 40:98103

502. Chate RA (1996) Evaluation of a dental practice cardiopulmonary resuscitation training scheme. Br Dent J 181:416-420

503. Atherton GJ, Pemberton MN, Thornhill MH (2000) Medical emergencies: the experience of staff of a UK dental teaching hospital. Br Dent J 188:320324

504. Sand M, Bechara FG, Sand D, Mann B (2009) Surgical and medical emergencies on board European aircraft: a retrospective study of 10189 cases. Crit Care 13:R3

505. Graf J, Stuben U, Pump S (2012) In-flight medical emergencies. Dtsch Arztebl Int 109:591-601. (quiz 2)

506. Weinlich M, Nieuwkamp N, Stueben U, Marzi I, Walcher F (2009) Telemedical assistance for in-flight emergencies on intercontinental commercial aircraft. J Telemed Telecare 15:409413

507. Peterson DC, Martin-Gill C, Guyette FX et al (2013) Outcomes of medical emergencies on commercial airline flights. N Engl J Med 368:2075-2083

508. McLoughlin DC, Jenkins DI (2003) Aircrew periodic medical examinations. Occup Med (Lond) 53:11-14

509. Hung KK, Cocks RA, Poon WK, Chan EY, Rainer TH, Graham CA (2013) Medical volunteers in commercial flight medical diversions. Aviat Space Environ Med 84:491-497 
510. Valani R, Cornacchia M, Kube D (2010) Flight diversions due to onboard medical emergencies on an international commercial airline. Aviat Space Environ Med 81:1037-1040

511. O'Rourke MF, Donaldson E, Geddes JS (1997) An airline cardiac arrest program. Circulation 96:2849-2853

512. Page RL, Joglar JA, Kowal RC et al (2000) Use of automated external defibrillators by a U.S. airline. N Engl J Med 343:1210-1216

513. Brown AM, Rittenberger JC, Ammon CM, Harrington S, Guyette FX (2010) In-flight automated external defibrillator use and consultation patterns. Prehosp Emerg Care 14:235-239

514. Bertrand C, Rodriguez Redington $P$, Lecarpentier E et al (2004) Preliminary report on AED deployment on the entire Air France commercial fleet: a joint venture with Paris XII University Training Programme. Resuscitation 63:175-181

515. Hunter A (1980) Will you volunteer in-flight medical care? Can Med Assoc J 123:137-140

516. Emergency Medical Equipment Training, Advisory Circular No. 121-34B. 2006. http:// www.faa.gov/documentLibrary/media/ Advisory_Circular/AC121-34B.pdf

517. Hinkelbein J, Neuhaus C, Wetsch WA et al (2014) Emergency medical equipment on board German airliners. J Travel Med 21:318-323

518. Emergency Medical Equipment, Advisory Circular No. 121-33B. 2006. http://www.faa.gov/ documentLibrary/media/Advisory_Circular/ AC121-33B.pdf

519. Commission Regulation (EC) No $859 / 2008$ of 20 August 2008 amending Council Regulation (EEC) No 3922/91 as regards common technical requirements and administrative procedures applicable to commercial transportation by aeroplane. Official Journal of the European Union, 2008. http://eur-lex.europa.eu/legal-content/EN/ ALL/?uri=CELEX:32008R0859

520. Sand M, Gambichler T, Sand D, Thrandorf C, Altmeyer P, Bechara FG (2010) Emergency medical kits on board commercial aircraft: a comparative study. Travel Med Infect Dis 8:388394

521. Skogvoll E, Bjelland E, Thorarinsson B (2000) Helicopter emergency medical service in outof-hospital cardiac arrest-a 10-year populationbased study. Acta Anaesthesiol Scand 44:972979

522. Lyon RM, Nelson MJ (2013) Helicopter emergency medical services (HEMS) response to out-of-hospital cardiac arrest. Scand J Trauma Resusc Emerg Med 21:1

523. Rittenberger JC, Hostler DP, Tobin T, Gaines J, Callaway CW (2008) Predictors of ROSC in witnessed aeromedical cardiac arrests. Resuscitation 76:43-46

524. Pietsch U, Lischke V, Pietsch C (2014) Benefit of mechanical chest compression devices in mountain HEMS: lessons learned from 1 year of experience and evaluation. Air Med J 33:299-301

525. Omori K, Sato S, Sumi Y et al (2013) The analysis of efficacy for AutoPulse system in flying helicopter. Resuscitation 84:1045-1050

526. Harmon KG, Asif IM, Klossner D, Drezner JA (2011) Incidence of sudden cardiac death in National Collegiate Athletic Association athletes. Circulation 123:1594-1600

527. Chandra N, Papadakis M, Sharma S (2010) Preparticipation screening of young competitive athletes for cardiovascular disorders. Phys Sportsmed 38:54-63
528. Angelini $P$ (2007) Coronary artery anomalies: an entity in search of an identity. Circulation 115:1296-1305

529. Maron BJ, Doerer JJ, Haas TS, Tierney DM, Muelle FO (2009) Sudden deaths in young competitive athletes: analysis of 1866 deaths in the United States, 1980-2006. Circulation 119:1085-1092

530. Maron BJ, Gohman TE, Kyle SB, Estes NA 3rd, Link MS (2002) Clinical profile and spectrum of commotio cordis. JAMA 287:1142-1146

531. Maron BJ, Haas TS, Ahluwalia A, Garberich RF, Estes NA 3rd, Link MS (2013) Increasing survival rate from commotio cordis. Heart Rhythm 10:219-223

532. Maron BJ, Friedman RA, Kligfield P et al (2014) Assessment of the 12-lead electrocardiogram as a screening test for detection of cardiovascular disease in healthy general populations of young people (12-25 years of age): a scientific statement from the American Heart Association and the American College of Cardiology. J Am Coll Cardiol 64:1479-1514

533. Lin CY, Wang YF, Lu TH, Kawach I (2015) Unintentional drowning mortality, by age and body of water: an analysis of 60 countries. Inj Prev 21:e43-e50

534. Venema AM, Groothoff JW, Bierens JJ (2010) The role of bystanders during rescue and resuscitation of drowning victims. Resuscitation 81:434-439

535. Szpilman D, Webber J, Quan L et al (2014) Creating a drowning chain of survival. Resuscitation 85:1149-1152

536. Bierens J (2014) Drowning Prevention, Rescue Treatment, 2. Aufl. Springer, Heidelberg

537. (2014) Global Report on Drowning. Preventing a Leading Killer. http://apps.who.int/iris/bitstream/ 10665/143893/1/9789241564786_eng.pdf

538. Racz E, Konczol F, Meszaros H et al (2015) Drowning-related fatalities during a 5-year period (2008-2012) in South-West Hungary-a retrospective study. J Forensic Leg Med 31:7-11

539. Halik R, Poznanska A, Seroka W, Wojtyniak B (2014) Accidental drownings in Poland in 2000 2012. Przegl Epidemiol 68:493-499, 591-494

540. Claesson A, Lindqvist J, Ortenwall P, Herlitz $J$ (2012) Characteristics of lifesaving from drowning as reported by the Swedish Fire and Rescue Services 1996-2010. Resuscitation 83:1072-1077

541. Idris AH, Berg RA, Bierens J et al (2003) Recommended guidelines for uniform reporting of data from drowning: The "Utstein style". Resuscitation 59:45-57

542. Idris AH, Berg RA, Bierens J et al (2003) Recommended guidelines for uniform reporting of data from drowning the "Utstein styleH. Circulation 108:2565-2574

543. Layon AJ, Modell JH (2009) Drowning: update 2009. Anesthesiology 110:1390-1401

544. Szpilman D, Bierens JJ, Handley AJ, Orlowski JP (2012) Drowning. N Engl J Med 366:2102-2110

545. Szpilman D, Soares M (2004) In-water resuscitation-is it worthwhile? Resuscitation 63:25-31

546. Quan L, Wentz KR, Gore EJ, Copass MK (1990) Outcome and predictors of outcome in pediatric submersion victims receiving prehospital care in King County, Washington. Pediatrics 86:586-593

547. Mtaweh $\mathrm{H}$, Kochanek PM, Carcillo JA, Bell MJ, Fink EL (2015) Patterns of multiorgan dysfunction after pediatric drowning. Resuscitation 90:91-96
548. Kyriacou DN, Arcinue EL, Peek C, Kraus JF (1994) Effect of immediate resuscitation on children with submersion injury. Pediatrics 94:137-142

549. Szpilman D (1997) Near-drowning and drowning classification: a proposal to stratify mortality based on the analysis of 1,831 cases. Chest 112:660-665

550. Wallis BA, Watt $K$, Franklin RC, Taylor $M$, Nixon JW, Kimble RM (2015) Interventions associated with drowning prevention in children and adolescents: systematic literature review. Inj Prev 21:195-204

551. Leavy JE, Crawford G, Portsmouth L et al (2015) Recreational drowning prevention interventions for adults, 1990-2012: a review. J Community Health 40:725-735

552. Vahatalo R, Lunetta P, Olkkola KT, Suominen PK (2014) Drowning in children: utstein style reporting and outcome. Acta Anaesthesiol Scand 58:604-610

553. Claesson A, Lindqvist J, Herlitz J (2014) Cardiac arrest due to drowning-changes over time and factors of importance for survival. Resuscitation 85:644-648

554. Dyson K, Morgans A, Bray J, Matthews B, Smith K (2013) Drowning related out-of-hospital cardiac arrests: characteristics and outcomes. Resuscitation 84:1114-1118

555. Bierens JJ, van der Velde EA, van Berkel M, van Zanten JJ (1990) Submersion in The Netherlands: prognostic indicators and results of resuscitation. Ann Emerg Med 19:1390-1395

556. Franklin RC, Pearn JH (2011) Drowning for love: the aquatic victim-instead-of-rescuer syndrome: drowning fatalities involving those attempting to rescue a child. J Paediatr Child Health 47:44-47

557. Perkins GD, Travers AH, Considine J et al (2015) Part 3: Adult basic life support and automated external defibrillation: 2015 international consensus on cardiopulmonary resuscitation and emergency cardiovascular care science with treatment recommendations. Resuscitation 95:e43-e70

558. Tipton MJ, Golden FS (2011) A proposed decision-making guide for the search, rescue and resuscitation of submersion (head under) victims based on expert opinion. Resuscitation 82:819824

559. Wanscher M, Agersnap L, Ravn J et al (2012) Outcome of accidental hypothermia with or without circulatory arrest: experience from the Danish Praesto Fjord boating accident. Resuscitation 83:1078-1084

560. Kieboom JK, Verkade HJ, Burgerhof JG et al (2015) Outcome after resuscitation beyond $30 \mathrm{~min}$ in drowned children with cardiac arrest and hypothermia: Dutch nationwide retrospective cohort study. BMJ 350:h418

561. Perkins GD (2005) In-water resuscitation: a pilot evaluation. Resuscitation 65:321-324

562. Winkler BE, Eff AM, Ehrmann U et al (2013) Effectiveness and safety of in-water resuscitation performed by lifeguards and laypersons: a crossover manikin study. Prehosp Emerg Care 17:409415

563. Watson RS, Cummings P, Quan L, Bratton S, Weiss NS (2001) Cervical spine injuries among submersion victims. J Trauma 51:658-662

564. March NF, Matthews RC (1980) Feasibility study of CPR in the water. Undersea Biomed Res 7:141148 
565. March NF, Matthews RC (1980) New techniques in external cardiac compressions. Aquatic cardiopulmonary resuscitation. JAMA 244:12291232

566. Barcala-Furelos R, Abelairas-Gomez C, RomoPerez V, Palacios-Aguilar J (2013) Effect of physical fatigue on the quality CPR: a water rescue study of lifeguards: physical fatigue and quality CPR in a water rescue. Am J Emerg Med 31:473-477

567. Claesson A, Karlsson T, Thoren AB, Herlitz J (2011) Delay and performance of cardiopulmonary resuscitation in surf lifeguards after simulated cardiac arrest due to drowning. Am J Emerg Med 29:1044-1050

568. Manolios N, Mackie I (1988) Drowning and neardrowning on Australian beaches patrolled by life-savers: a 10-year study, 1973-1983. Med J Aust 148:165-167, 70-71

569. Baker PA, Webber JB (2011) Failure to ventilate with supraglottic airways after drowning. Anaesth Intensive Care 39:675-677

570. Montenij LJ, de Vries W, Schwarte L, Bierens JJ (2011) Feasibility of pulse oximetry in the initial prehospital management of victims of drowning: a preliminary study. Resuscitation 82:1235-1238

571. Moran I, Zavala E, Fernandez R, Blanch L, Mancebo J (2003) Recruitment manoeuvres in acute lung injury/acute respiratory distress syndrome. Eur Respir J Suppl 42:37 s-42 s

572. Wyatt JP, Tomlinson GS, Busuttil A (1999) Resuscitation of drowning victims in south-east Scotland. Resuscitation 41:101-104

573. Bolte RG, Black PG, Bowers RS, Thorne JK, Corneli HM (1988) The use of extracorporeal rewarming in a child submerged for $66 \mathrm{~min}$. JAMA 260:377379

574. Schmidt U, Fritz KW, Kasperczyk W, Tscherne $H$ (1995) Successful resuscitation of a child with severe hypothermia after cardiac arrest of 88 min. Prehospital Disaster Med 10:60-62

575. Oehmichen M, Hennig R, Meissner C (2008) Neardrowning and clinical laboratory changes. Leg Med (Tokyo) 10:1-5

576. Modell JH (1985) Serum electrolyte changes in near-drowning victims. JAMA 253:557

577. Gregorakos L, Markou N, Psalida V et al (2009) Near-drowning: clinical course of lung injury in adults. Lung 187:93-97

578. The Acute Respiratory Distress Syndrome Network (2000) Ventilation with lower tidal volumes as compared with traditional tidal volumes for acute lung injury and the acute respiratory distress syndrome. N Engl J Med 342:1301-1308

579. Sutherasan Y, Penuelas O, Muriel A et al (2015) Management and outcome of mechanically ventilated patients after cardiac arrest. Crit Care 19:215

580. Eich C, Brauer A, Timmermann A et al (2007) Outcome of 12 drowned children with attempted resuscitation on cardiopulmonary bypass: an analysis of variables based on the "Utstein Style for Drowning". Resuscitation 75:42-52

581. Guenther U, Varelmann D, Putensen C, Wrigge $H$ (2009) Extended therapeutic hypothermia fo several days during extracorporeal membraneoxygenation after drowning and cardiac arrest Two cases of survival with no neurological sequelae. Resuscitation 80:379-381

582. Kim KI, Lee WY, Kim HS, Jeong JH, Ko HH (2014) Extracorporeal membrane oxygenation in neardrowning patients with cardiac or pulmonary failure. Scand J Trauma Resusc Emerg Med 22:77
583. Champigneulle B, Bellenfant-Zegdi F, Follin A et al (2015) Extracorporeal life support (ECLS) for refractory cardiac arrest after drowning: an 11-year experience. Resuscitation 88:126-131

584. Wood C (2010) Towards evidence based emergency medicine: best BETs from the Manchester Royal Infirmary. BET 1: prophylactic antibiotics in near-drowning. Emerg Med J 27:393-394

585. Van Berkel MB, Lie RLK et al (1996) Pulmonary oedema, pneumonia and mortality in submersion victims a retrospective study in 125 patients. Inten Care Med 22:101-107

586. Davies KJ, Walters JH, Kerslake IM, Greenwood R, Thomas MJ (2013) Early antibiotics improve survival following out-of hospital cardiac arrest. Resuscitation 84:616-619

587. Tadie JM, Heming N, Serve E et al (2012) Drowning associated pneumonia: a descriptive cohort. Resuscitation 83:399-401

588. (2005) Proceedings of the 2005 International Consensus on Cardiopulmonary Resuscitation and Emergency Cardiovascular Care Science with Treatment Recommendations. Resuscitation 67:157-341

589. Paal P, Ellerton J, Sumann $G$ et al (2007) Basic life support ventilation in mountain rescue. Official recommendations of the International Commission for Mountain Emergency Medicine (ICAR MEDCOM). High Alt Med Biol 8:147-154

590. Elsensohn F, Soteras I, Resiten O, Ellerton J, Brugger H, Paal P (2011) Equipment of medical backpacks in mountain rescue. High Alt Med Bio 12:343-347

591. Elsensohn F, Agazzi G, Syme D et al (2006) The use of automated external defibrillators and public access defibrillators in the mountains: official guidelines of the international commission for mountain emergency medicine ICAR-MEDCOM. Wilderness Environ Med 17:64-66

592. Brugger H, Elsensohn F, Syme D, Sumann G, Falk $M$ (2005) A survey of emergency medical services in mountain areas of Europe and North America: official recommendations of the International Commission for Mountain Emergency Medicine (ICAR Medcom). High Alt Med Biol 6:226-237

593. Tomazin I, Ellerton J, Reisten O, Soteras I, Avbelj M (2011) International Commission for Mountain Emergency M. Medical standards for mountain rescue operations using helicopters: official consensus recommendations of the International Commission for Mountain Emergency Medicine (ICAR MEDCOM). High Alt Med Biol 12:335-341

594. Pietsch U, Lischke V, Pietsch C, Kopp KH (2014) Mechanical chest compressions in an avalanche victim with cardiac arrest: an option for extreme mountain rescue operations. Wilderness Environ Med 25:190-193

595. Ellerton J, Gilbert H (2012) Should helicopters have a hoist or ,long-line' capability to perform mountain rescue in the UK? Emerg Med J 29:5659

596. Klemenc-Ketis Z, Tomazin I, Kersnik J (2012) HEMS in Slovenia: one country, four models, different quality outcomes. Air Med J 31:298-304

597. Tomazin I, Vegnuti M, Ellerton J, Reisten O, Sumann G, Kersnik J (2012) Factors impacting on the activation and approach times of helicopter emergency medical services in four Alpine countries. Scand J Trauma Resusc Emerg Med 20:56

598. Wang JC, Tsai SH, Chen YL et al (2014) The physiological effects and quality of chest compressions during CPR at sea level and high altitude. Am J Emerg Med 32:1183-1188
599. Suto T, Saito S (2014) Considerations for resuscitation at high altitude in elderly and untrained populations and rescuers. Am J Emerg Med 32:270-276

600. Narahara H, Kimura M, Suto T et al (2012) Effects of cardiopulmonary resuscitation at high altitudes on the physical condition of untrained and unacclimatized rescuers. Wilderness Environ Med 23:161-164

601. Boyd J, Brugger H, Shuster M (2010) Prognostic factors in avalanche resuscitation: a systematic review. Resuscitation 81:645-652

602. Locher T, Walpoth BH (1996) [Differential diagnosis of circulatory failure in hypothermic avalanche victims: retrospective analysis of 32 avalanche accidents]. Praxis (Bern 1994) 85:1275-1282

603. Grissom CK, Radwin Ml, Scholand MB, Harmston $\mathrm{CH}$, Muetterties MC, Bywater TJ (2004) Hypercapnia increases core temperature cooling rate during snow burial. J Appl Physiol 96:13651370

604. Oberhammer R, Beikircher W, Hormann C et al (2008) Full recovery of an avalanche victim with profound hypothermia and prolonged cardiac arrest treated by extracorporeal re-warming. Resuscitation 76:474-480

605. Mair P, Brugger H, Mair B, Moroder L, Ruttmann $E$ (2014) Is extracorporeal rewarming indicated in avalanche victims with unwitnessed hypothermic cardiorespiratory arrest? High Alt Med Biol 15:500-503

606. Boue Y, Payen JF, Brun J et al (2014) Survival after avalanche-induced cardiac arrest. Resuscitation 85:1192-1196

607. Hilmo J, Naesheim T, Gilbert M (2014) „Nobody is dead until warm and dead": prolonged resuscitation is warranted in arrested hypothermic victims also in remote areas-a retrospective study from northern Norway. Resuscitation 85:1204-1211

608. Brugger H, Sumann G, Meister R et al (2003) Hypoxia and hypercapnia during respiration into an artificial air pocket in snow: implications for avalanche survival. Resuscitation 58:81-88

609. Haegeli P, Falk M, Brugger H, Etter HJ, Boyd $J$ (2011) Comparison of avalanche survival patterns in Canada and Switzerland. CMAJ 183:789-795

610. Boyd J, Haegeli P, Abu-Laban RB, Shuster M, Butt JC (2009) Patterns of death among avalanche fatalities: a 21-year review. CMAJ 180:507-512

611. Brugger H, Durrer B, Elsensohn F et al (2013) Resuscitation of avalanche victims: Evidencebased guidelines of the international commission for mountain emergency medicine (ICAR MEDCOM): intended for physicians and other advanced life support personnel. Resuscitation 84:539-546

612. Brugger H, Paal P, Boyd J (2011) Prehospital resuscitation of the buried avalanche victim. High Alt Med Biol 12:199-205

613. Kottmann A, Blancher M, Spichiger T et al (2015) The Avalanche Victim Resuscitation Checklist, a new concept for the management of avalanche victims. Resuscitation 91:e7-e8

614. Budnick LD (1984) Bathtub-related electrocutions in the United States (1979) to 1982. JAMA 252:918-920

615. (1998) Lightning-associated deaths - United States, 1980-1995. MMWR Morb Mortal Wkly Rep 47:391-394 
616. Geddes LA, Bourland JD, Ford G (1986) The mechanism underlying sudden death from electric shock. Med Instrum 20:303-315

617. Zafren K, Durrer B, Herry JP, Brugger H (2005) Lightning injuries: prevention and on-site treatment in mountains and remote areas. Official guidelines of the International Commission for Mountain Emergency Medicine and the Medical Commission of the International Mountaineering and Climbing Federation (ICAR and UIAA MEDCOM). Resuscitation 65:369-372

618. Cherington M (1995) Lightning injuries. Ann Emerg Med 25:517-519

619. Fahmy FS, Brinsden MD, Smith J, Frame JD (1999) Lightning: the multisystem group injuries. J Trauma 46:937-940

620. Patten BM (1992) Lightning and electrical injuries. Neurol Clin 10:1047-1058

621. Browne BJ, Gaasch WR (1992) Electrical injuries and lightning. Emerg Med Clin North Am 10:211-229

622. Kleiner JP, Wilkin JH (1978) Cardiac effects of lightning stroke. JAMA 240:2757-2759

623. Lichtenberg R, Dries D, Ward K, Marshall W, Scanlon P (1993) Cardiovascular effects of lightning strikes. J Am Coll Cardiol 21:531-536

624. Cooper MA (1995) Emergent care of lightning and electrical injuries. Sem Neurol 15:268-278

625. Milzman DP, Moskowitz L, Hardel M (1999) Lightning strikes at a mass gathering. South Med J 92:708-710

626. Cooper MA (1980) Lightning injuries: prognostic signs for death. Ann Emerg Med 9:134-138

627. Kleinschmidt-DeMasters BK (1995) Neuropathology of lightning-strike injuries. Sem Neurol 15:323-328

628. Cherington M, McDonough G, Olson S, Russon $R$, Yarnell PR (2007) Lichtenberg figures and lightning: case reports and review of the literature. Cutis 80:141-143

629. Epperly TD, Stewart JR (1989) The physical effects of lightning injury. J Fam Pract 29:267-272

630. Duclos PJ, Sanderson LM (1990) An epidemiological description of lightning-related deaths in the United States. Int J Epidemiol 19:673-679

631. Whitcomb D, Martinez JA, Daberkow D (2002) Lightning injuries. South Med J 95:1331-1334

632. Goldman RD, Einarson A, Koren G (2003) Electric shock during pregnancy. Can Fam Physician 49:297-298

633. Blumenthal R, Saayman G (2014) Bone marrow embolism to the lung in electrocution: two case reports. Am J Foren Med Pathol 35:170-171

634. El Sayed M, Tamim H, Mann NC (2015) Description of procedures performed on patients by emergency medical services during mass casualty incidents in the United States. Am J Emerg Med 33:1030-1036

635. World DR (2014) 2014. https://www.ifrc.org/ world-disasters-report-2014/data

636. Schenk E, Wijetunge G, Mann NC, Lerner EB, Longthorne A, Dawson D (2014) Epidemiology of mass casualty incidents in the United States. Prehosp Emerg Care 18:408-416

637. Tokuda Y, Kikuchi M, Takahashi O, Stein GH (2006) Prehospital management of sarin nerve gas terrorism in urban settings: 10 years of progress after the Tokyo subway sarin attack. Resuscitation 68:193-202

638. Lamhaut L, Dagron C, Apriotesei R et al (2010) Comparison of intravenous and intraosseous access by pre-hospital medical emergency personnel with and without CBRN protective equipment. Resuscitation 81:65-68
639. Castle N, Pillay Y, Spencer N (2011) Comparison of six different intubation aids for use while wearing CBRN-PPE: a manikin study. Resuscitation 82:1548-1552

640. Castle N, Bowen J, Spencer N (2010) Does wearing CBRN-PPE adversely affect the ability for clinicians to accurately, safely, and speedily draw up drugs? Clin Toxicol (Phila) 48:522-527

641. Cross KP, Petry MJ, Cicero MX (2015) A better START for low-acuity victims: data-driven refinement of mass casualty triage. Prehosp Emerg Care 19:272-278

642. SALT mass casualty triage (2008) concept endorsed by the American College of Emergency Physicians, American College of Surgeons Committee on Trauma, American Trauma Society, National Association of EMS Physicians, National Disaster Life Support Education Consortium, and State and Territorial Injury Prevention Directors Association. Disaster Med Public Health Prep 2:245-246

643. Cone DC, Serra J, Burns K, MacMillan DS, Kurland L, Van Gelder C (2009) Pilot test of the SALT mass casualty triage system. Prehospital Emerg Care 13:536-540

644. Risavi BL, Terrell MA, Lee W, Holsten DL Jr (2013) Prehospital mass-casualty triage training-written versus moulage scenarios: how much do EMS providers retain? Prehosp Disaster Med 28:251256

645. Knight JF, Carley S, Tregunna B et al (2010) Serious gaming technology in major incident triage training: a pragmatic controlled trial. Resuscitation 81:1175-1179

646. Postma IL, Weel H, Heetveld MJ et al (2013) Mass casualty triage after an airplane crash near Amsterdam. Injury 44:1061-1067

647. Jones N, White ML, Tofil N et al (2014) Randomized trial comparing two mass casualty triage systems (JumpSTART versus SALT) in a pediatric simulated mass casualty event Prehospital Emerg Care 18:417-423

648. Masoli M, Fabian D, Holt S, Beasley R (2004) The global burden of asthma: executive summary of the GINA Dissemination Committee report. Allergy 59:469-478

649. To T, Stanojevic S, Moores G et al (2012) Global asthma prevalence in adults: findings from the cross-sectional world health survey. BMC Public Health 12:204

650. Pearce N, Ait-Khaled N, Beasley R et al (2007) Worldwide trends in the prevalence of asthma symptoms: phase III of the International Study of Asthma and Allergies in Childhood (ISAAC). Thorax 62:758-766

651. Anandan C, Nurmatov U, van Schayck OC, Sheikh A (2010) Is the prevalence of asthma declining? Systematic review of epidemiological studies. Allergy 65:152-167

652. Cohen S, Berkman N, Avital A et al (2015) Decline in asthma prevalence and severity in Israel over 10-year period. Respiration 89:27-32

653. Mikalsen IB, Skeiseid L, Tveit LM, Engelsvold DH, Oymar K (2015) Decline in admissions for childhood asthma, a 26-year period populationbased study. Pediatr Allergy Immunol

654. Chung KF, Wenzel SE, Brozek JL et al (2014) International ERS/ATS guidelines on definition, evaluation and treatment of severe asthma. Eur Respir J 43:343-373

655. Romagnoli M, Caramori G, Braccioni F et al (2007) Near-fatal asthma phenotype in the ENFUMOSA Cohort. Clin Exp Allergy 37:552-557
656. Alvarez GG, Schulzer M, Jung D, Fitzgerald JM (2005) A systematic review of risk factors associated with near-fatal and fatal asthma. Can Respir J 12:265-270

657. Turner MO, Noertjojo K, Vedal S, Bai T, Crump S, Fitzgerald JM (1998) Risk factors for near-fatal asthma: a case-control study in hospitalized patients with asthma. Am J Respir Crit Care Med 157:1804-1809

658. Ernst P, Spitzer WO, Suissa S et al (1992) Risk of fatal and near-fatal asthma in relation to inhaled corticosteroid use. JAMA 268:3462-3464

659. Suissa S, Blais L, Ernst P (1994) Patterns of increasing beta-agonist use and the risk of fatal or near-fatal asthma. Eur Respir J 7:1602-1609

660. Alvarez GG, Fitzgerald JM (2007) A systematic review of the psychological risk factors associated with near fatal asthma or fatal asthma. Respiration 74:228-236

661. Sturdy PM, Victor CR, Anderson HR et al (2002) Psychological, social and health behaviour risk factors for deaths certified as asthma: a national case-control study. Thorax 57:1034-1039

662. Roberts G, Patel N, Levi-Schaffer F, Habibi P, Lack G (2003) Food allergy as a risk factor for life-threatening asthma in childhood: a casecontrolled study. J Allergy Clin Immunol 112:168-174

663. Why asthmastillkills: the national review of asthma deaths (NRAD). Confidential Enquiry Report 2014. 2014. http://www.rcplondon.ac.uk/ sites/default/files/why-asthma-still-kills-fullreport.pdf

664. Tsai CL, Lee WY, Hanania NA, Camargo CA Jr (2012) Age-related differences in clinical outcomes for acute asthma in the United States, 2006-2008. J Allergy Clin Immunol 129:1252$1258 \mathrm{e} 1$

665. Williams TJ, Tuxen DV, Scheinkestel CD, Czarny D, Bowes G (1992) Risk factors for morbidity in mechanically ventilated patients with acute severe asthma. Am Rev Respir Dis 146:607-615

666. Kokturk N, Demir N, Kervan F, Dinc E, Koybasioglu A, Turktas H (2004) A subglottic mass mimicking near-fatal asthma: a challenge of diagnosis. J Emerg Med 26:57-60

667. Global Strategy for Asthma Management and Prevention (2009) 2009. (Accessed 24/06/10, 2010

668. SIGN 141 British guideline on the management of asthma. 2014. http://www.sign.ac.uk/pdf/ SIGN141.pdf

669. Rodrigo GJ, Nannini LJ (2006) Comparison between nebulized adrenaline and beta2 agonists for the treatment of acute asthma. A meta-analysis of randomized trials. Am J Emerg Med 24:217-222

670. Rodrigo G, Rodrigo C, Burschtin O (1999) A metaanalysis of the effects of ipratropium bromide in adults with acute asthma. Am J Med 107:363370

671. Aaron SD (2001) The use of ipratropium bromide for the management of acute asthma exacerbation in adults and children: a systematic review. J Asthma 38:521-530

672. Mohammed S, Goodacre S (2007) Intravenous and nebulised magnesium sulphate for acute asthma: systematic review and meta-analysis. Emerg Med J 24:823-830

673. Powell C, Dwan K, Milan SJ et al (2012) Inhaled magnesium sulfate in the treatment of acute asthma. Cochrane Database Syst Rev 12:CD003898 
674. Bradshaw TA, Matusiewicz SP, Crompton GK, Innes JA, Greening AP (2008) Intravenous magnesium sulphate provides no additive benefit to standard management in acute asthma. Respir Med 102:143-149

675. Goodacre S, Cohen J, Bradburn M et al (2013) Intravenous or nebulised magnesium sulphate versus standard therapy for severe acute asthma (3Mg trial): a double-blind, randomised controlled trial. Lancet Respir Med 1:293-300

676. Kew KM, Kirtchuk L, Michell Cl (2014) Intravenous magnesium sulfate for treating adults with acute asthma in the emergency department. Cochrane Database Syst Rev 5:CD010909

677. Rowe BH, Spooner CH, Ducharme FM, Bretzlaff JA, Bota GW (2001) Corticosteroids for preventing relapse following acute exacerbations of asthma. Cochrane Database Syst Rev 2001:CD000195

678. Ratto D, Alfaro C, Sipsey J, Glovsky MM, Sharma OP (1988) Are intravenous corticosteroids required in status asthmaticus? JAMA 260:527529

679. Travers A, Jones AP, Kelly K, Barker SJ, Camargo CA, Rowe BH (2001) Intravenous beta2-agonists for acute asthma in the emergency department. Cochrane Database Syst Rev 2001:CD002988

680. Cowman S, Butler J (2008) Towards evidence based emergency medicine: best BETs from the Manchester Royal Infirmary. BET 3. The use of intravenous aminophylline in addition to betaagonists and steroids in acute asthma. Emerg Med J 25:289-290

681. Parameswaran K, Belda J, Rowe BH (2000) Addition of intravenous aminophylline to beta2agonists in adults with acute asthma. Cochrane Database Syst Rev 2000:CD002742

682. Kuitert LM, Watson D (2007) Antileukotrienes as adjunctive therapy in acute asthma. Drugs 67:1665-1670

683. Camargo CA Jr, Gurner DM, Smithline HA et al (2010) A randomized placebo-controlled study of intravenous montelukast for the treatment of acute asthma. J Allergy Clin Immunol 125:374380

684. Watts K, Chavasse RJ (2012) Leukotriene receptor antagonists in addition to usual care for acute asthma in adults and children. Cochrane Database Syst Rev 5:CD006100

685. Rodrigo GJ, Rodrigo C, Pollack CV, Rowe B (2003) Use of helium-oxygen mixtures in the treatment of acute asthma: a systematic review. Chest 123:891-896

686. Gupta D, Keogh B, Chung KF et al (2004) Characteristics and outcome for admissions to adult, general critical care units with acute severe asthma: a secondary analysis of the ICNARC Case Mix Programme Database. Crit Care 8:R112-R121

687. Brenner B, Corbridge T, Kazzi A (2009) Intubation and mechanical ventilation of the asthmatic patient in respiratory failure. J Allergy Clin Immunol 124:S19-S28

688. Antonelli M, Pennisi MA, Montini L (2005) Clinical review: Noninvasive ventilation in the clinical setting-experience from the past 10 years. Crit Care 9:98-103

689. Lim WJ, Mohammed Akram R, Carson KV et al (2012) Non-invasive positive pressure ventilation for treatment of respiratory failure due to severe acute exacerbations of asthma. Cochrane Database Systematic Rev 12:CD004360
690. Leatherman JW, McArthur C, Shapiro RS (2004) Effect of prolongation of expiratory time on dynamic hyperinflation in mechanically ventilated patients with severe asthma. Crit Care Med 32:1542-1545

691. Bowman FP, Menegazzi JJ, Check BD, Duckett TM (1995) Lower esophageal sphincter pressure during prolonged cardiac arrest and resuscitation. Ann Emerg Med 26:216-219

692. Lapinsky SE, Leung RS (1996) Auto-PEEP and electromechanical dissociation. N Engl J Med 335:674

693. Rogers PL, Schlichtig R, Miro A, Pinsky M (1991) Auto-PEEP during CPR. An "occult" cause of electromechanical dissociation? Chest 99:492493

694. Rosengarten PL, Tuxen DV, Dziukas L, Scheinkestel C, Merrett K, Bowes G (1991) Circulatory arrest induced by intermittent positive pressure ventilation in a patient with severe asthma. Anaesth Intensive Care 19:118121

695. Sprung J, Hunter K, Barnas GM, Bourke DL (1994) Abdominal distention is not always a sign of esophageal intubation: cardiac arrest due to „auto-PEEP". Anesth Analg 78:801-804

696. Harrison R (2010) Chest compression first aid for respiratory arrest due to acute asphyxic asthma. Emerg Med J 27:59-61

697. Deakin CD, McLaren RM, Petley GW, Clewlow F, Dalrymple-Hay MJ (1998) Effects of positive endexpiratory pressure on transthoracic impedanceimplications for defibrillation. Resuscitation 37:9-12

698. Galbois A, Ait-Oufella H, Baudel JL et al (2010) Pleural ultrasound compared to chest radiographic detection of pneumothorax resolution after drainage. Chest 138(3):648-655

699. Mabuchi N, Takasu H, Ito S et al (1991) Successful extracorporeal lung assist (ECLA) for a patient with severe asthma and cardiac arrest. Clin Inten Care 2:292-294

700. Martin GB, Rivers EP, Paradis NA, Goetting MG Morris DC, Nowak RM (1998) Emergency department cardiopulmonary bypass in the treatment of human cardiac arrest. Chest 113:743-751

701. Mabvuure NT, Rodrigues JN (2014) External cardiac compression during cardiopulmonary resuscitation of patients with left ventricular assist devices. Interact Cardiovasc Thorac Surg 19:286-289

702. Hubner P, Meron G, Kurkciyan I et al (2014) Neurologic causes of cardiac arrest and outcomes. J Emerg Med 47:660-667

703. Thurman DJ, Hesdorffer DC, French JA (2014) Sudden unexpected death in epilepsy: assessing the public health burden. Epilepsia 55:14791485

704. Arnaout M, Mongardon N, Deye N et al (2015) Out-of-hospital cardiac arrest from brain cause: epidemiology, clinical features, and outcome in a multicenter cohort*. Crit Care Med 43:453-460

705. Skrifvars MB, Parr MJ (2012) Incidence, predisposing factors, management and survival following cardiac arrest due to subarachnoid haemorrhage: a review of the literature. Scand J Trauma Resusc Emerg Med 20:75

706. Mitsuma W, Ito M, Kodama M et al (2011) Clinical and cardiac features of patients with subarachnoid haemorrhage presenting with out-ofhospital cardiac arrest. Resuscitation 82:12941297
707. Sandroni C, Dell'Anna AM (2015) Out-of-hospital cardiac arrest from neurologic cause: recognition and outcome* ${ }^{*}$. Crit Care Med 43:508-509

708. Noritomi DT, de Cleva R, Beer I et al (2006) Doctors awareness of spontaneous subarachnoid haemorrhage as a cause of cardiopulmonary arrest. Resuscitation 71:123-124

709. Sandroni C, Adrie C, Cavallaro F et al (2010) Are patients brain-dead after successful resuscitation from cardiac arrest suitable as organ donors? A systematic review. Resuscitation 81:1609-1614

710. Jain R, Nallamothu BK, Chan PS (2010) American Heart Association National Registry of Cardiopulmonary Resuscitation i. Body mass index and survival after in-hospital cardiac arrest. Circ Cardiovasc Qual Outcomes 3:490-497

711. Testori C, Sterz F, Losert H et al (2011) Cardiac arrest survivors with moderate elevated body mass index may have a better neurological outcome: a cohort study. Resuscitation 82:869873

712. Obesity and overweight. Fact sheet No 311. 2015. http://www.who.int/mediacentre/ factsheets/fs311/en/

713. Adams KF, Schatzkin A, Harris TB et al (2006) Overweight, obesity, and mortality in a large prospective cohort of persons 50 to 71 years old. N Engl J Med 355:763-778

714. Romero-Corral A, Montori VM, Somers VK et al (2006) Association of bodyweight with total mortality and with cardiovascular events in coronary artery disease: a systematic review of cohort studies. The Lancet 368:666-678

715. Adabag S, Huxley RR, Lopez FL et al (2015) Obesity related risk of sudden cardiac death in the atherosclerosis risk in communities study. Heart 101:215-221

716. Duflou J, Virmani R, Rabin I, Burke A, Farb A, Smialek J (1995) Sudden death as a result of heart disease in morbid obesity. Am Heart J 130:306-313

717. Nishisaki A, Maltese MR, Niles DE et al (2012) Backboards are important when chest compressions are provided on a soft mattress. Resuscitation 83:1013-1020

718. Bunch TJ, White RD, Lopez-Jimenez F, Thomas RJ (2008) Association of body weight with total mortality and with ICD shocks among survivors of ventricular fibrillation in out-of-hospital cardiac arrest. Resuscitation 77:351-355

719. White RD, Blackwell TH, Russell JK, Jorgenson DB (2004) Body weight does not affect defibrillation, resuscitation, or survival in patients with out-of-hospital cardiac arrest treated with a nonescalating biphasic waveform defibrillator. Crit Care Med 32:S387-S392

720. Sugerman $H$, Windsor $A$, Bessos M, Wolfe $L$ (1997) Intra-abdominal pressure, sagittal abdominal diameter and obesity comorbidity. J Intern Med 241:71-79

721. Holmberg TJ, Bowman SM, Warner KJ et al (2011) The association between obesity and difficult prehospital tracheal intubation. Anesth Analg 112:1132-1138

722. Reminiac F, Jouan Y, Cazals X, Bodin JF, Dequin PF, Guillon A (2014) Risks associated with obese patient handling in emergency prehospital care. Prehosp Emerg Care 18:555-557

723. Kruska P, Kappus S Kerner T (2012) [Obesity in prehospital emergency care]. Anasthesiol Intensivmed Notfallmed Schmerzther 47:556562 
724. Chalkias A, Xanthos T (2014) The obesity paradox in cardiac arrest patients. Int J Cardiol 171:101102

725. Trends in Maternal Mortality: 1990 to 2013. Estimates by WHO, UNICEF, UNFPA, The World Bank and the United Nations Population Division 2013. http://www.who.int/reproductivehealth/ publications/monitoring/maternalmortality-2013/en/

726. Lipman S, Cohen S, Einav S et al (2014) The Society for Obstetric Anesthesia and Perinatology consensus statement on the management of cardiac arrest in pregnancy. Anesth Analg 118:1003-1016

727. Soar J, Callaway CW, Aibiki M et al (2015) Part 4 advanced life support: 2015 International Consensus on Cardiopulmonary Resuscitation and Emergency Cardiovascular Care Science With Treatment Recommendations. Resuscitation $\mathrm{XX}: \mathrm{XX}$

728. Say L, Chou D, Gemmill A et al (2014) Global causes of maternal death: a WHO systematic analysis. Lancet Glob Health 2:e323-e333

729. UK and Ireland Confidential Enquiries into Maternal Deaths and Morbidity 2009-2012. Saving Lives, Improving Mothers' Care 2014

730. Page-Rodriguez A, Gonzalez-Sanchez JA (1999) Perimortem cesarean section of twin pregnancy: case report and review of the literature. Acad Emerg Med 6:1072-1074

731. Cardosi RJ, Porter KB (1998) Cesarean delivery of twins during maternal cardiopulmonary arrest. Obstet Gynecol 92:695-697

732. Mendonca C, Griffiths J, Ateleanu B, Collis RE (2003) Hypotension following combined spinalepidural anaesthesia for Caesarean section. Left lateral position vs. tilted supine position. Anaesthesia 58:428-431

733. Rees SG, Thurlow JA, Gardner IC, Scrutton MJ, Kinsella SM (2002) Maternal cardiovascular consequences of positioning after spinal anaesthesia for Caesarean section: left 15 degree table tilt vs. left lateral. Anaesthesia 57:15-20

734. Bamber JH, Dresner M (2003) Aortocaval compression in pregnancy: the effect of changing the degree and direction of lateral tilt on maternal cardiac output. Anesth Analg 97:256-258. (table of contents)

735. Carbonne B, Benachi A, Leveque ML, Cabrol D, Papiernik E (1996) Maternal position during labor: effects on fetal oxygen saturation measured by pulse oximetry. Obstet Gynecol 88:797-800

736. Tamas P, Szilagyi A, Jeges S et al (2007) Effects of maternal central hemodynamics on fetal heart rate patterns. Acta Obstet Gynecol Scand 86:711-714

737. Abitbol MM (1985) Supine position in labor and associated fetal heart rate changes. Obstet Gynecol 65:481-486

738. Kinsella SM (2003) Lateral tilt for pregnant women: why 15 degrees? Anaesthesia 58:835836

739. Goodwin AP, Pearce AJ (1992) The human wedge. A manoeuvre to relieve aortocaval compression during resuscitation in late pregnancy. Anaesthesia 47:433-434

740. Rees GA, Willis BA (1988) Resuscitation in late pregnancy. Anaesthesia 43:347-349

741. Jones SJ, Kinsella SM, Donald FA (2003) Comparison of measured and estimated angles of table tilt at Caesarean section. Br J Anaesth 90:86-87
742. Nanson J, Elcock D, Williams M, Deakin CD (2001) Do physiological changes in pregnancy change defibrillation energy requirements? Br J Anaesth 87:237-239

743. Chiloiro M, Darconza G, Piccioli E, De Carne M, Clemente C, Riezzo G (2001) Gastric emptying and orocecal transit time in pregnancy. J Gastroenterol 36:538-543

744. O'Sullivan G (1993) Gastric emptying during pregnancy and the puerperium. Int J Obstet Anesth 2:216-224

745. Johnson MD, Luppi CJ, Over DC (1998) Cardiopulmonary Resuscitation. In: Gambling DR, Douglas MJ (Hrsg) Obstetric Anesthesia and Uncommon Disorders. W. B. Saunders, Philadelphia, S 51-74

746. Izci B, Vennelle M, Liston WA, Dundas KC, Calder AA, Douglas NJ (2006) Sleep-disordered breathing and upper airway size in pregnancy and post-partum. Eur Respir J 27:321-327

747. Rahman K, Jenkins JG (2005) Failed tracheal intubation in obstetrics: no more frequent but still managed badly. Anaesthesia 60:168-171

748. Henderson JJ, Popat MT, Latto IP Pearce AC (2004) Difficult Airway Society guidelines for management of the unanticipated difficult intubation. Anaesthesia 59:675-694

749. Potts M, Prata N, Sahin-Hodoglugil NN (2010) Maternal mortality: one death every $7 \mathrm{~min}$. The Lancet 375:1762-1763

750. Lewis Ge. The Confidential Enquiry into Maternal and Child Health (CEMACH). Saving Mothers Lives; Reviewing Maternal Deaths to Make Motherhood Safer (2003)-05. The Seventh Report of the United Kingdom Confidential Enquiries into Maternal Deaths in the United Kingdom. London: CEMACH, 2007. London: RCOG Press; 2007

751. Gynecologists ACoOa (2012) Optimizing Protocols in Obstetrics management of obstetric hemmorhage

752. Organization WH (2012) WHO recommendation for the prevention and treatment of postpartum haemorrhage

753. Geoghegan J, Daniels JP, Moore PA, Thompson PJ, Khan KS, Gulmezoglu AM (2009) Cell salvage at caesarean section: the need for an evidencebased approach. BJOG 116:743-747

754. Bouwmeester FW, Bolte AC, van Geijn HP (2005) Pharmacological and surgical therapy for primary postpartum hemorrhage. Curr Pharm Des 11:759-773

755. Hofmeyr GJ, Abdel-Aleem H, Abdel-Aleem MA (2008) Uterine massage for preventing postpartum haemorrhage. Cochrane Database Syst Rev 16:CD006431

756. Sekhavat L, Tabatabaii A, Dalili M, Farajkhoda T, Tafti AD (2009) Efficacy of tranexamic acid in reducing blood loss after cesarean section. $J$ Matern Fetal Neonatal Med 22:72-75

757. Phillips LE, McLintock C, Pollock W et al (2009) Recombinant activated factor VII in obstetric hemorrhage: experiences from the Australian and New Zealand Haemostasis Registry. Anesth Analg 109:1908-1915

758. Bomken C, Mathai S, Biss T, Loughney A, Hanley J (2009) Recombinant Activated Factor $\mathrm{VII}$ (rFVIla) in the Management of Major Obstetric Haemorrhage: a case series and a proposed guideline for use. Obstet Gynecol Int 2009:364843
759. Doumouchtsis SK, Papageorghiou AT, Vernier C, Arulkumaran S (2008) Management of postpartum hemorrhage by uterine balloon tamponade: prospective evaluation of effectiveness. Acta Obstet Gynecol Scand 87:849-855

760. Georgiou C (2009) Balloon tamponade in the management of postpartum haemorrhage: a review. BJOG 116:748-757

761. El-Hamamy E CBL (2005) A worldwide review of the uses of the uterine compression suture techniques as alternative to hysterectomy in the management of severe post-partum haemorrhage. J Obstet Gynaecol 25:143-149

762. Hong TM, Tseng HS, Lee RC, Wang JH, Chang CY (2004) Uterine artery embolization: an effective treatment for intractable obstetric haemorrhage. Clin Radiol 59:96-101

763. Knight $M$ (2007) Peripartum hysterectomy in the UK: management and outcomes of the associated haemorrhage. BJOG 114:1380-1387

764. Rossi AC, Lee RH, Chmait RH (2010) Emergency postpartum hysterectomy for uncontrolled postpartum bleeding: a systematic review. Obstet Gynecol 115:637-644

765. Yu S, Pennisi JA, Moukhtar M, Friedman EA (1995) Placental abruption in association with advanced abdominal pregnancy. A case report. J Reprod Med 40:731-735

766. Ray P, Murphy GJ, Shutt LE (2004) Recognition and management of maternal cardiac disease in pregnancy. Br J Anaesth 93:428-439

767. Abbas AE, Lester SJ, Connolly H (2005) Pregnancy and the cardiovascular system. International J Cardiol 98:179-189

768. Royal College of Obstetricians and Gynaecologists. Cardiac disease in pregnancy 2011

769. James AH, Jamison MG, Biswas MS, Brancazio LR Swamy GK, Myers ER (2006) Acute myocardial infarction in pregnancy: a United States population-based study. Circulation 113:15641571

770. Ahearn GS, Hadjiliadis D, Govert JA, Tapson VF (2002) Massive pulmonary embolism during pregnancy successfully treated with recombinant tissue plasminogen activator: a case report and review of treatment options. Arch Intern Med 162:1221-1227

771. Drenthen W, Pieper PG, Roos-Hesselink JW et al (2007) Outcome of pregnancy in women with congenital heart disease: a literature review. J Am Coll Cardiol 49:2303-2311

772. Sibai B, Dekker G, Kupferminc M (2005) Preeclampsia. The Lancet 365:785-799

773. Sibai BM (2005) Diagnosis, prevention, and management of eclampsia. Obstet Gynecol 105:402-410

774. Duley L, Gulmezoglu AM, Henderson-Smart DJ (2003) Magnesium sulphate and other anticonvulsants for women with pre-eclampsia. Cochrane Database Syst Rev 2003:CD000025

775. Duley L, Henderson-Smart D (2003) Magnesium sulphate versus phenytoin for eclampsia. Cochrane Database Syst Rev 2003:CD000128

776. Duley L, Henderson-Smart D (2003) Magnesium sulphate versus diazepam for eclampsia. Cochrane database Syst Rev 2003:CD000127

777. World Health Organization. WHO recommendations for Prevention and treatment of pre-eclampsia and eclampsia2011 
778. Duley L, Henderson-Smart DJ, Walker GJ, Chou D (2010) Magnesium sulphate versus diazepam for eclampsia. Cochrane Database Syst Rev 2010:CD000127

779. Duley L, Henderson-Smart DJ, Chou D (2010) Magnesium sulphate versus phenytoin for eclampsia. Cochrane Database Syst Rev 2010:CD000128

780. Duley L, Gulmezoglu AM, Chou D (2010) Magnesium sulphate versus lytic cocktail for eclampsia. Cochrane Database Syst Rev 2010:CD002960

781. Duley L, Matar HE, Almerie MQ, Hall DR (2010) Alternative magnesium sulphate regimens for women with pre-eclampsia and eclampsia. Cochrane Database Syst Rev 2010:CD007388

782. Knight $M$ (2008) Antenatal pulmonary embolism: risk factors, management and outcomes. BJOG 115:453-461

783. Dapprich M, Boessenecker W (2002) Fibrinolysis with alteplase in a pregnant woman with stroke. Cerebrovasc Dis 13:290

784. Turrentine MA, Braems G, Ramirez MM (1995) Use of thrombolytics for the treatment of thromboembolic disease during pregnancy. Obstet Gynecol Surv 50:534-541

785. Thabut G, Thabut D, Myers RP et al (2002) Thrombolytic therapy of pulmonary embolism: a meta-analysis. J Am Coll Cardiol 40:1660-1667

786. Patel RK, Fasan O, Arya R (2003) Thrombolysis in pregnancy. Thromb Haemost 90:1216-1217

787. Conde-Agudelo A, Romero R (2009) Amniotic fluid embolism: an evidence-based review. Am J Obstet Gynecol 201:445 e1-13

788. Fitzpatrick K, Tuffnell D, Kurinczuk J, Knight M (2015) Incidence, risk factors, management and outcomes of amniotic-fluid embolism: a population-based cohort and nested casecontrol study. BJOG

789. Stanten RD, Iverson LI, Daugharty TM, Lovett SM, Terry C, Blumenstock E (2003) Amniotic fluid embolism causing catastrophic pulmonary vasoconstriction: diagnosis by transesophageal echocardiogram and treatment by cardiopulmonary bypass. Obstet Gynecol 102:496-498

790. Einav S, Kaufman N, Sela HY (2012) Maternal cardiac arrest and perimortem caesarean delivery: evidence or expert-based? Resuscitation 83:1191-1200

791. Dijkman A, Huisman CM, Smit M et al (2010) Cardiac arrest in pregnancy: increasing use of perimortem caesarean section due to emergency skills training? Bjog 117:282-287

792. Baghirzada L, Balki M (2013) Maternal cardiac arrest in a tertiary care centre during 1989-2011: a case series. Can J Anaesth 60:1077-1084

793. Katz VL, Dotters DJ, Droegemueller W (1986) Perimortem cesarean delivery. Obstet Gynecol 68:571-576

794. American Heart Association in collaboration with International Liaison Committee on Resuscitation (2000) Guidelines 2000 for Cardiopulmonary Resuscitation and Emergency Cardiovascular Care. Circulation 102(suppl):11-1384

795. Chapter 4; Part 6: Cardiac Arrest Associated with Pregnancy. In: Cummins R, Hazinski M, Field J, eds. ACLS-The Reference Textbook. Dallas: American Heart Association; 2003:143-58

796. Katz V, Balderston K, DeFreest M (2005) Perimortem cesarean delivery: were our assumptions correct? Am J Obstet Gynecol 192:1916-1920 (discussion 20-21)
797. Oates S, Williams GL, Rees GA (1988) Cardiopulmonary resuscitation in late pregnancy. BMJ 297:404-405

798. Strong THJ, Lowe RA (1989) Perimortem cesarean section. Am J Emerg Med 7:489-494

799. Boyd R, Teece S (2002) Towards evidence based emergency medicine: best BETs from the Manchester Royal Infirmary. Perimortem caesarean section. Emerg Med J 19:324-325

800. Allen MC, Donohue PK, Dusman AE (1993) The limit of viability - neonatal outcome of infants born at 22 to 25 weeks' gestation. N Engl J Med 329:1597-1601

801. Moore C, Promes SB (2004) Ultrasound in pregnancy. Emerg Med Clin North Am 22:697-722

802. Rittenberger JC, Kelly E, Jang D, Greer K, Heffner A (2008) Successful outcome utilizing hypothermia after cardiac arrest in pregnancy: a case report. Crit Care Med 36:1354-1356

803. Natale A, Davidson T, Geiger MJ, Newby K (1997) Implantable cardioverter-defibrillators and pregnancy: a safe combination? Circulation 96:2808-2812

804. Siassakos D, Crofts JF, Winter C, Weiner CP, Draycott TJ (2009) The active components of effective training in obstetric emergencies. BJOG 116:1028-1032

805. Siassakos D, Bristowe K, Draycott TJ et al (2011) Clinical efficiency in a simulated emergency and relationship to team behaviours: a multisite cross-sectional study. BJOG 118:596-607

806. McNally B, Robb R, Mehta M et al (2011) Out-ofHospital Cardiac Arrest Surveillance - Cardiac Arrest Registry to Enhance Survival (CARES), United States, October 1, 2005-December 31, 2010. MMWR Surveill Summ 60:1-19

807. Chugh SS, Jui J, Gunson K et al (2004) Current burden of sudden cardiac death: multiple source surveillance versus retrospective death certificate-based review in a large U.S community. J Am Coll Cardiol 44:1268-1275

808. Churpek MM, Yuen TC, Winslow C, Hall J, Edelson DP (2015) Differences in vital signs between elderly and nonelderly patients prior to ward cardiac arrest. Critical Care Med 43:816-822

809. Van Hoeyweghen RJ,B, Mullie A et al (1992) Survival after out-of-hospital cardiac arrest in elderly patients. Belgian Cerebral Resuscitation Study Group. Ann Emerg Med 21:1179-1184

810. Tung P, Albert CM (2013) Causes and prevention of sudden cardiac death in the elderly. Nature reviews. Cardiology 10:135-142

811. Teodorescu C, Reinier K, Dervan C et al (2010) Factors associated with pulseless electric activity versus ventricular fibrillation: the Oregon sudden unexpected death study. Circulation 122:21162122

812. Winther-Jensen M, Pellis T, Kuiper M et al (2015) Mortality and neurological outcome in the elderly after target temperature management for out-of-hospital cardiac arrest. Resuscitation 91:92-98

813. Lamantia MA, Stewart PW, Platts-Mills TF et al (2013) Predictive value of initial triage vital signs for critically ill older adults. West J Emerg Med 14:453-460

814. Nasa P, Juneja D, Singh O (2012) Severe sepsis and septic shock in the elderly: an overview. World J Crit Care Med 1:23-30

815. Tresch DD (1998) Management of the older patient with acute myocardial infarction: difference in clinical presentations between older and younger patients. J Am Geriatr Soc 46:1157-1162
816. Tresch DD (1996) Signs and Symptoms of Heart Failure in Elderly Patients. Am J Geriatr Cardiol 5:27-33

817. Gardin JM, Arnold AM, Bild DE et al (1998) Left ventricular diastolic filling in the elderly: the cardiovascular health study. Am J Cardiol $82: 345-351$

818. Priebe $\mathrm{HJ}$ (2000) The aged cardiovascular risk patient. Br J Anaesth 85:763-778

819. Hasegawa K, Hagiwara Y, Imamura T et al (2013) Increased incidence of hypotension in elderly patients who underwent emergency airway management: an analysis of a multi-centre prospective observational study. Int J Emerg Med 6:12

820. Montamat SC, Cusack BJ, Vestal RE (1989) Management of drug therapy in the elderly. $\mathrm{N}$ Engl J Med 321:303-309

821. Black CJ, Busuttil A, Robertson C (2004) Chest wall injuries following cardiopulmonary resuscitation. Resuscitation 63:339-343

822. Krischer JP, Fine EG, Davis JH, Nagel EL (1987) Complications of cardiac resuscitation. Chest 92:287-291

823. Kashiwagi Y, Sasakawa T, Tampo A et al (2015) Computed tomography findings of complications resulting from cardiopulmonary resuscitation. Resuscitation 88:86-91

824. Grimaldi D, Dumas F, Perier MC et al (2014) Shortand long-term outcome in elderly patients after out-of-hospital cardiac arrest: a cohort study. Critical care medicine 42:2350-2357

825. Nolan JP, Soar J, Smith GB et al (2014) Incidence and outcome of in-hospital cardiac arrest in the United Kingdom National Cardiac Arrest Audit. Resuscitation 85:987-992

826. Deasy C, Bray JE, Smith K et al (2011) Out-ofhospital cardiac arrests in the older age groups in Melbourne, Australia. Resuscitation 82:398-403

827. Chan PS, Nallamothu BK, Krumholz HM et al (2013) Long-term outcomes in elderly survivors of in-hospital cardiac arrest. N Engl J Med 368:1019-1026

828. van deGEM, van Munster BC, van deWFT, van Delden JJ, Scholten RJ, Hooft L (2013) Pre-arrest predictors of survival after resuscitation from out-of-hospital cardiac arrest in the elderly a systematic review. BMC Geriatr 13:68

829. Menon PR, Ehlenbach WJ, Ford DW, Stapleton RD (2014) Multiple in-hospital resuscitation efforts in the elderly. Crit Care Med 42:108-117

830. Bunch TJ, White RD, Khan AH, Packer DL (2004) Impact of age on long-term survival and quality of life following out-of-hospital cardiac arrest. Crit Care Med 32:963-967

831. Boyd K, Teres D, Rapoport J, Lemeshow S (1996) The relationship between age and the use of DNR orders in critical care patients. Evidence for age discrimination. Arch Intern Med 156:1821-1826

832. Schwenzer KJ, Smith WT, Durbin CG Jr (1993) Selective application of cardiopulmonary resuscitation improves survival rates. Anesth Analg 76:478-484

833. Seder DB, Patel N, McPherson J et al (2014) Geriatric experience following cardiac arrest at six interventional cardiology centers in the United States 2006-2011: interplay of age, donot-resuscitate order, and outcomes. Crit Care Med 42:289-295 